\title{
Tactile Feedback for \\ Myoelectric Forearm
}

\section{Prostheses}

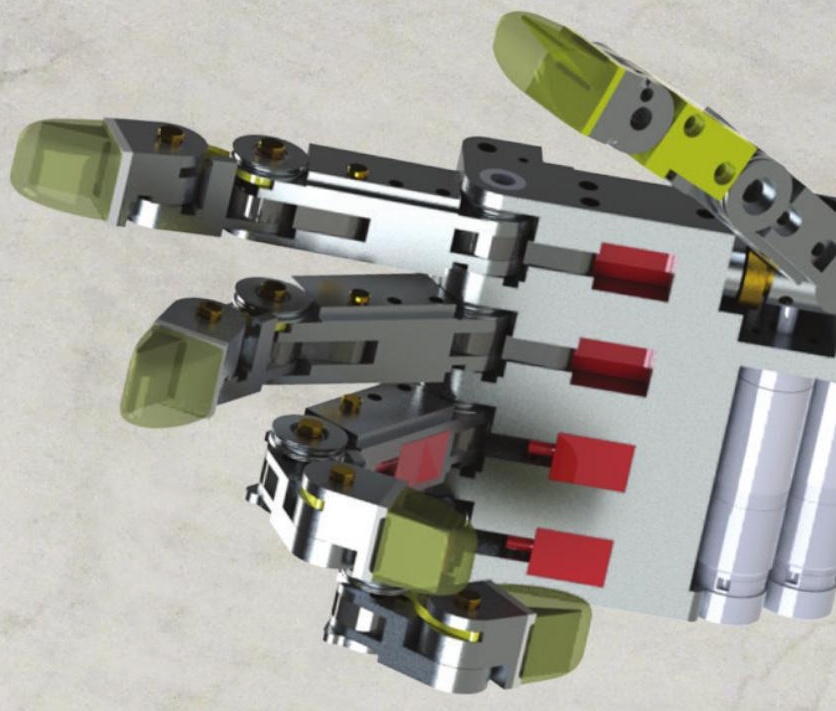

Heidi Witteveen 
TACTILE FEEDBACK FOR MYOELECTRIC FOREARM PROSTHESES 


\section{Members of the graduation committee:}

Chairman and secretary:

Prof. dr. ir. A.J. Mouthaan

Promotors:

Prof. dr. ir. P.H. Veltink

Prof. dr. J.S. Rietman

Members:

Prof. dr. R.J.A. van Wezel

Prof. dr. ir. H.F.J.M. Koopman

Prof. dr. ir. S. Stramigioli

Prof. dr. C.K. van der Sluis

Dr. ir. D.H. Plettenburg
University of Twente, Enschede, The Netherlands

University of Twente, Enschede, The Netherlands University of Twente, Enschede, The Netherlands Roessingh Research and Development, Enschede

University of Twente, Enschede, The Netherlands University of Twente, Enschede, The Netherlands University of Twente, Enschede, The Netherlands University Medical Center Groningen, The Netherlands Technical University Delft, The Netherlands

This work is supported by the Ministry of Economic Affairs (Pieken in de Delta Oost Nederland), the Netherlands under grant PID082035/1.6.1b

Publication of this thesis is financially supported by:

Biomedical Signals and Systems, University of Twente

UNIVERSITY OF TWENTE.

TMS International BV, Oldenzaal

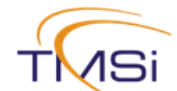

This work was carried out at the MIRA Institute for Biomedical Technology and Technical Medicine, University of Twente, in collaboration with Roessingh Research and Development, The Netherlands.

Cover design: Heidi Witteveen, original painting by Michelangelo

3D-render on cover: Bart Peerdeman

Author: Heidi Witteveen

Email: h.j.b.witteveen@utwente.nl

ISBN: 978-90-365-3588-5

DOI: $10.3990 / 1.9789036535885$

Printed by:

Gildeprint Drukkerijen 


\title{
TACTILE FEEDBACK FOR MYOELECTRIC FOREARM PROSTHESES
}

\author{
PROEFSCHRIFT
}

ter verkrijging van

de graad van doctor aan de Universiteit Twente, op gezag van de rector magnificus, prof. dr. H. Brinksma, volgens besluit van het College voor Promoties in het openbaar te verdedigen op donderdag 6 februari 2014 om 16:45 uur

door

Heintje Johanna Berendina Witteveen geboren op 30 december 1983 te Apeldoorn, Nederland 
Dit proefschrift is goedgekeurd door:

Prof. dr. ir. P.H. Veltink (promotor)

Prof. dr. J.S. Rietman (promotor) 
Contents of the thesis

Part 1 - Basics of feedback

Introduction - Feedback for myoelectric forearm prostheses

Chapter 1 - Vibrotactile stimulation with a single coin motor

Part 2 - Evaluation of stimulation parameters

Chapter 2 - Vibro- and electrotactile user feedback on hand aperture

Chapter 3 - Vibrotactile hand aperture feedback and distraction

Chapter 4 - Vibrotactile grasping force and slip feedback

Chapter 5 - Combined vibrotactile hand aperture and grasping force feedback

Part 3 - Evaluation of feedback methods

Chapter 7 - Vibrotactile feedback and EMG control

Chapter 8 - Vibrotactile feedback for amputee and congenital defect patients

Chapter 9 - Daily life grasping performance with vibrotactile feedback

Chapter 9A - Daily life grasping performance of one amputee

References

Summary

Samenvatting

Dankwoord

Biography 


\section{Part 1}

\section{Basics of feedback}




\section{Introduction - Feedback for myoelectric forearm prostheses}

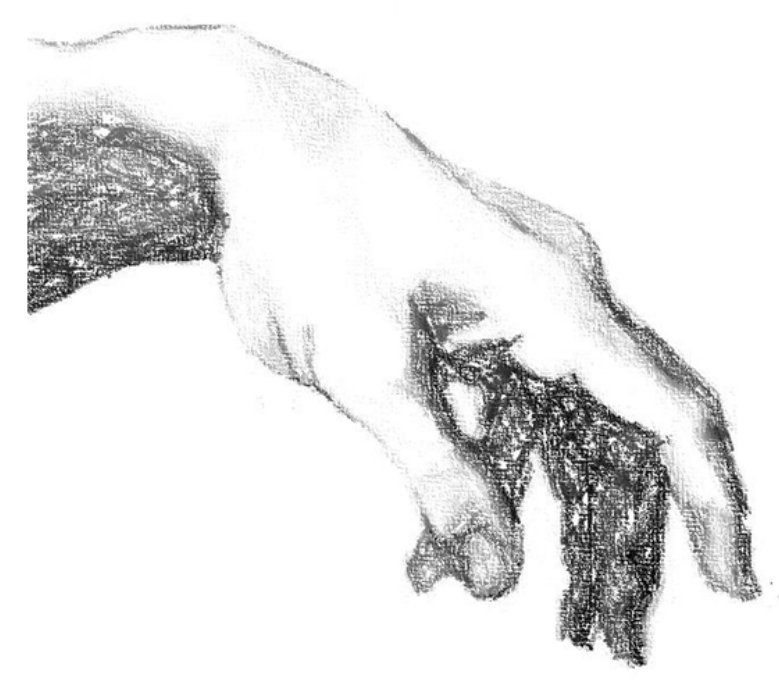

Parts adapted from: Myoelectric forearm prostheses; state of the art from a user centered perspective

Authors: Bart Peerdeman, Daphne Boere, Heidi Witteveen, Rianne Huis in 't Veld, Hermie Hermens, Stefano Stramigioli, Hans Rietman, Peter Veltink, Sarthak Misra

Published in: Journal of Rehabilitation Research and Development, vol. 48. no.6, pp. 719-738, 2011 
Aristotle (384 BC - $322 \mathrm{BC}$ ), one of the great Greek philosophers, once stated that the human hand can be considered as being the instrument of instruments. This statement still holds today, especially when looking at the wide range of functionalities of the human hand. The missing of a hand therefore greatly influences the physical capabilities of persons. Even though a large number of people returned to work $(>60 \%)$ after traumatic amputation, in most cases a change in job was necessary [45]. Not only their physical capabilities, but also their psychological and emotional well-being can be altered after upper limb amputation. In around $30-36 \%$ of the cases anxiety or border line symptoms were reported after traumatic upper limb amputation $[45,49]$ and in around $18-28 \%$ of the cases significant depression symptoms were reported.

In the USA, 10,000 upper limb amputations are being performed each year [105]. In the Netherlands this number lies between 40 and 60 per year [2] with a total number of 4000 people living in the Netherlands who miss an upper limb. Amputations at the forearm level form around $25 \%$ of these upper limb amputations [2].

(a)

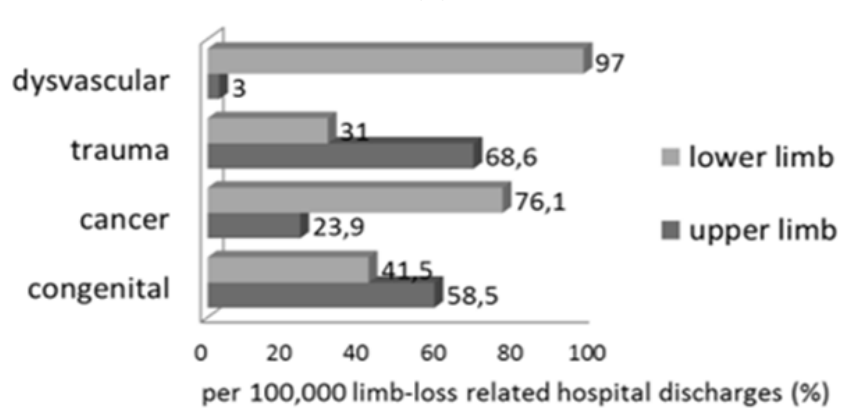

(b)

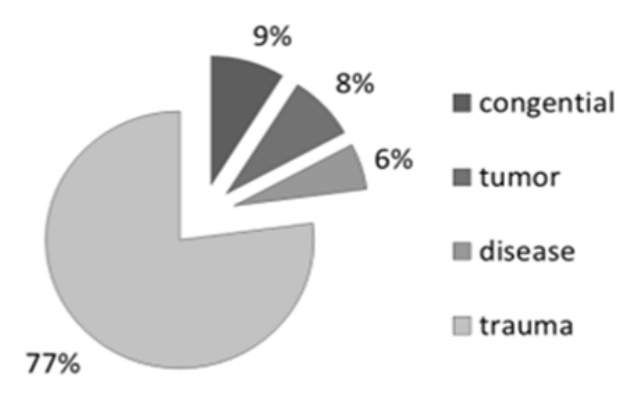

Figure 1: (a) Differences in causes of lower and upper limb amputations (adapted from the national limb loss information center, amputee coalition, USA, 2012) and (b) Percentages of causes of upper extremity amputation (adapted from biomed.brown.edu)

The most common causes of an amputation of the upper limb are traumatic accidents, which is in strong contrast to amputations of the lower limb, which are mostly caused by vascular problems often related to diabetes (see Figure 1a). This also results in a younger age at which the upper limb amputations are performed compared to the lower limb amputations: $60 \%$ of the upper limb amputations are performed at the age between 21 and 64 yrs. and $10 \%$ at the age below 21 yrs. Other reasons for amputation of the upper limb can be infections, tumors and nerve injuries (see Figure $1 \mathrm{~b}$ for an overview of the percentages of upper limb loss causes) [1].

In the USA, the percentage of people missing an upper limb due to congenital defects is around 9\% [1] and in the Netherlands each year around 36 children are born with a congenital defect of the upper limb. 


\section{Current forearm prostheses}

To compensate for the loss of an upper limb, a forearm prosthesis can be prescribed. The three main types of forearm prostheses are (1) cosmetic forearm prostheses, (2) body powered prostheses and (3) myoelectric forearm prostheses (see Figure 2).

(a)

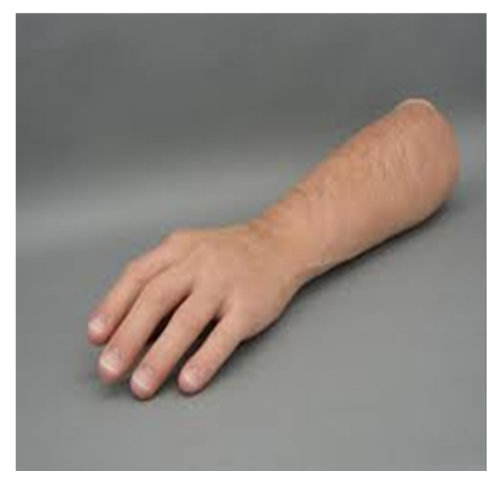

(b)

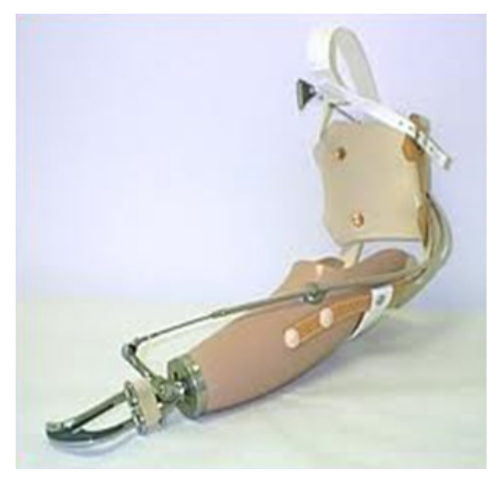

(c)

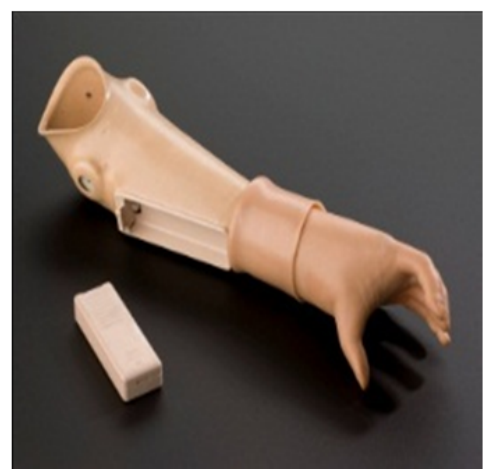

Figure 2: (a) cosmetic forearm prosthesis (picture adapted from http://www.silikontechnik.ch), (b) body-powered forearm prosthesis (picture adapted from http://rehabindy0.tripod.com) and (c) myoelectric forearm prosthesis (picture adapted from http://collectionsonline.nmsi.ac.uk/)

There are no clear numbers about the distribution of these types of prostheses over the population of possible prosthesis users, but cosmetic prostheses seem most popular and myoelectric prostheses by far the least popular $[12,45]$. Cosmetic covers can make the prosthetic hand look very human-like and therefore are easily accepted by the environment of the user. Cosmetics can be regarded as the most important function of the cosmetic forearm prosthesis, but another highly appreciated functionality is the supportive function during bimanual tasks. A higher level of functionality can be achieved with body-powered prostheses. The opening and closing of the prosthesis hand is controlled through small movements of the (opposing) shoulder via a harness. The hand aperture and grasping force of the prosthesis are directly related to the movement and the forces applied by the cables and thus the movement of the shoulder [111]. The grasping force that can be applied by these prostheses is strongly limited due to this direct relation between the grasping force and the force that can be applied by the shoulder. Furthermore, the harness is not very pleasant to wear and the functionality of the prosthesis is limited to one controllable degree of freedom, the opening and closing of the hand. Myoelectric forearm prostheses have the potential to offer a higher level of functionality, but the majority of the currently used prostheses still only provides the opening and closing of the hand. Myoelectric prostheses are controlled by muscle activity measured from the remaining forearm muscles. Dry EMG electrodes are placed above the wrist flexors and wrist extensors, which controls the hand closing and opening respectively. Myoelectric prostheses can offer proportional control of hand movement velocity and proportional control of grasping force during object holding. A higher level of muscle activation (higher EMG amplitude) results in a higher velocity or 
larger grasping force. In some prostheses, rotation (or even flexion) of the wrist is also included, which can be controlled by the same electrode pair as used for the hand aperture after a co-contraction command. To date, the state-of-the-art myoelectric forearm prostheses, the I-limb from Touch Bionics, the Michelangelo hand from Otto Bock and the Be-bionic hand from RSL Steeper offer the user more functionality by an increased number of grasps that can be performed by the prostheses. Furthermore, these prostheses offer more naturally looking grasping by folding the fingers around an object. Finger movements continue until an object is being touched. Still only two EMG electrodes are used in these prostheses and therefore different grasps have to be manually selected by placing the thumb in the desired position or are selected via cocontraction schemes. The main drawbacks of today's myoelectric forearm prostheses are their limited function (in comparison to the healthy hand), the lack of sensory feedback, the slow speed and especially the heavy weight of the prosthesis.

Recently, a couple of research projects have been started on the improvement of myoelectric forearm prostheses. One of the largest is the SmartHand project (www.smarthand.org), which is a European project with a large number of involved institutes. The goal of this project was "to design and develop a new, lightweight, dexterous, sensorized prosthetic hand with intrinsic actuation, able to be fitted in subjects with an amputation level, long below the elbow" [38]. They have been working on EMG control, but also looked into neural sensing. Furthermore, several concepts to provide sensory feedback were proposed. The focus of another project, the Fluidhand, was on the development of a light-weight prosthesis, which was achieved by the use of hydraulics [56]. Within this project also a simple force feedback system was developed. A complete different approach to increase the functionality of a myoelectric prosthesis (mainly for above the elbow amputations) is the use of targeted reinnervation. For this approach, the nerves that initially innervated the forearm muscles are transferred to the shoulder region, innervating the larger shoulder muscles, which activity can be easily measured with surface EMG. An additional benefit is the reinnervation of the skin above these nerves, which results in the perception of touch at this location that is perceived as touch of the hand [84].

\section{Need for feedback}

Although the functionality of myoelectric forearm prostheses improves continuously, the number of prescribed myoelectric forearm prostheses that is being used on a regular basis is low. About 20 to $34 \%$ of these prescribed prostheses is even completely rejected by their users $[18,45]$. Elaborate studies, using questionnaires, have been performed to find the underlying problems that lead to this high level of prosthesis rejection $[12,18$, 118]. In all these studies, the lack of sensory feedback was indicated as one of the major factors in prosthesis abandonment. Patients indicated that a prosthesis requiring less visual attention was preferred, which is an indirect indication of the need for artificial hand aperture feedback [12]. Furthermore, force feedback was indicated by patients as an important aspect to be incorporated in future prostheses $[86,118]$. In a study by 
Blank et al., it is shown that proprioceptive feedback about finger movements is required to achieve acceptable targeting accuracies with forearm prostheses [20].

From a control engineering point of view, myoelectric prostheses can be regarded openloop systems when visual feedback is not available [32, 110]. In these open-loop systems, the control input cannot be regulated continuously based on the performance of the system, because this sensory information is not available. Childress [32] defined three types of feedback that can be used in myoelectric prostheses to close the control loop and optimize the prosthesis control: type A feedback is the auditory and visual feedback, which is already present in current prostheses, but can be blocked or overwhelmed in certain situations. Type B feedback is sensory feedback (proprioceptive or tactile), which is usually provided through vibrotactile or electrotactile stimulation to the skin. Finally, type $C$ feedback is directly transmitted to the controller inside the prostheses. This type of feedback is used in most of the state-of-the-art commercial available prostheses, where object slippage is prevented by automatic grip force adjustments. A trade-off should be made between type B and type C feedback to optimize the prosthesis control, but still keep the prosthesis user in control of his prosthesis.

\section{Sensory substitution}

An important phenomenon following nerve injury, called brain plasticity, is the change in neural pathways and synapses, which can even lead to cortical remapping. After amputation of the forearm the ascending and descending pathways are disrupted and as a consequence brain plasticity occurs to a greater or lesser extent. Plasticity after amputation can have positive effects like a higher sensitivity of the skin of the stump to compensate for the loss of input. On the other hand, a negative effect of plasticity after amputation can be the development of phantom limb sensations [51]. It is stated that the use of a prosthetic system can enhance the positive plasticity effects and diminish the negative effects $[51,152]$. As a result of brain plasticity, other intact sensory pathways can be used for substitution of the sensory pathways that are not available anymore in amputee patients. This redirection of sensory information is called sensory substitution [14]. In the case of forearm amputations, the skin of the stump can be used to provide the artificial sensory feedback. Several studies have evaluated the effect of artificial sensory feedback on the embodiment of a prosthesis, which is related to the incorporation of the prosthesis as part of human body. It was shown that embodiment is increased with touch feedback provided to the forearm stump [94] as well as with vibrotactile feedback provided to the fingertips of healthy subjects [43]. Another way to investigate or improve the embodiment of the prosthesis is the use of rubber hand illusion experiments. In healthy subjects, the touch of a rubber hand can be experienced as the perception of touch at the own hand when both the rubber hand and the hand of the subject are being touched simultaneously for some time [21]. This phenomenon was also demonstrated for amputees, where, after a period of synchronous touching of the rubber hand and the forearm stump, touch at the forearm stump was perceived as touch 
at the fingertip of the rubber hand [54]. The rubber hand became more or less part of the body, which can possibly be used to increase the embodiment of a prosthetic hand. Another phenomenon, also related to brain plasticity, that can occur after amputation is the phantom map, which is the redirection of lost sensory perception to the forearm (touch of the forearm can be felt like touch to the fingers). This phenomenon is not always present and not all patients show clear phantom maps. In a study by Antfolk et al. it was shown that vibrotactile stimulation to these phantom map positions results in better feedback discrimination [9].

\section{Myopro project}

The low percentage of prescribed myoelectric forearm prostheses that is being used on a regular basis has triggered the start of the Myopro project. The main goals of this project are based on the shortcomings of today's myoelectric prostheses and are therefore formulated as follows: (1) to improve the mechanical and control characteristics of the prosthetic hand by using underactuation and innovative control schemes, (2) to improve the user control of a myoelectric forearm prosthesis by increasing the number of degrees of freedom using multichannel surface electromyography, (3) to develop a natural and intuitive feedback mechanism and (4) to develop a virtual reality training program to enable aimed early-phase rehabilitation. The project consortium is formed by three commercial companies and three research groups (IMS, Re-lion, TMSi, RRD, UT-RAM and UT-BSS). IMS (Integrated Mechanization Solutions, Almelo, the Netherlands) is mainly involved in the development of microneedle array electrodes and the integration of all subsystems. Re-lion (Enschede, the Netherlands) is responsible for the development of the virtual training program and TMSi (Twente Medical Systems International, Oldenzaal, the Netherlands) is involved in the implementation of the classification algorithms and the technical evaluation of the integrated test setup. The main contribution of the RRD (Roessingh Research and Development, Enschede, the Netherlands) is the development of a sensing algorithm that classifies the surface EMG data into different grasps. The Robotics and Mechatronics research group of the University of Twente (UT-RAM) is involved in the development of the mechanical prototype of the prosthesis and the control of the prosthesis. The Biomedical Signals and Systems research group of the University of Twente is responsible for the development of an intuitive feedback system, which is the focus of this PhD-thesis.

\section{State-of-the-art of feedback}

The first step in the Myopro project was the establishment of the functional requirements for a future myoelectric prosthesis. This was achieved by combining the outcomes from a workshop with representative forearm prosthesis users and information from literature $[12,18,113]$. The structure of the needs assessment method used to derive the functional requirements is given in Figure 3.

The workshop participants consisted of a multidisciplinary group (9 men and 10 women) of representative users and engineers from multiple centers throughout the 
Netherlands. All participants had interests and expertise in the area of upper-limb amputation and prostheses. The representative users were two occupational therapists, three rehabilitation medicine physicians, two physiotherapists, a certified prosthetist/orthotist, and a movement scientist. Six researchers and four engineers constituted the academic contributors.

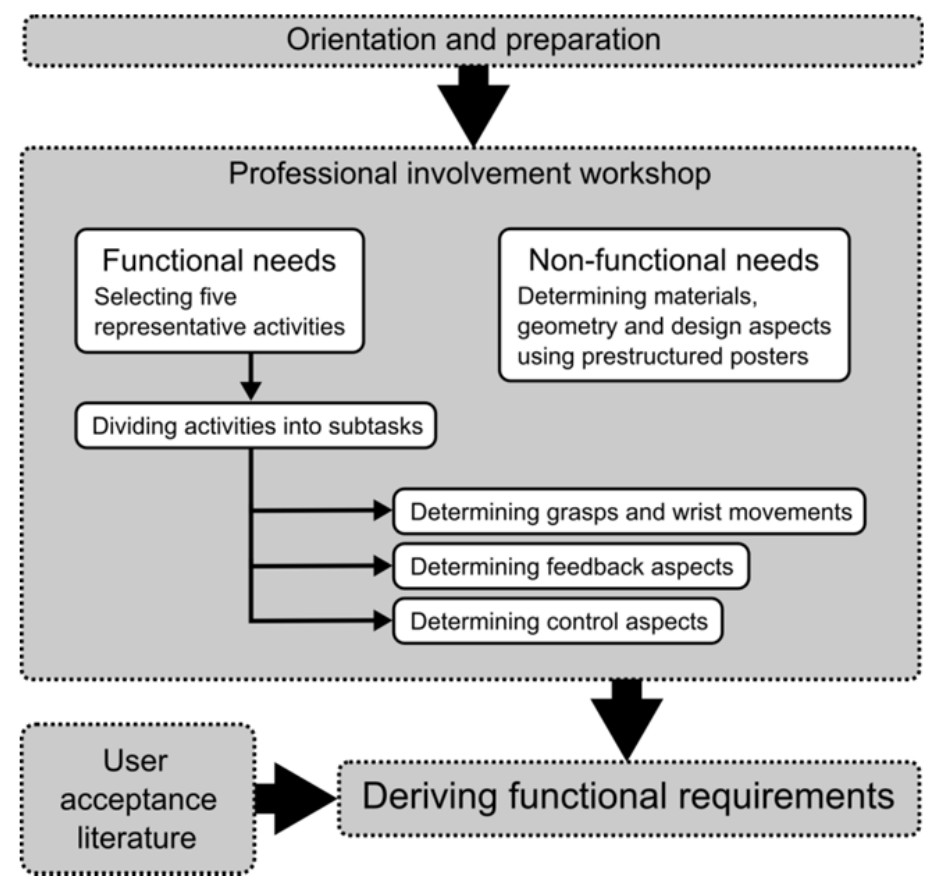

Figure 3: Overview of needs assessment approach, including internal structure of the workshop

A plenary discussion led to a selection of five activities in which the important aspects of upper-limb prosthesis use are well represented. Each activity was examined, focusing on three prosthesis subsystems; (1) sensing, (2) control and (3) feedback and analyzed using a structured worksheet specially designed for this workshop. Multidisciplinary groups were each asked to divide one activity into subtasks. For every subsystem, the worksheet contained several questions to be answered for each subtask of the activity. For the feedback aspect these questions were: "Which information would the user need during each part of the activity?", "What are the requirements for feedback during each part of the activity?" and "How should the information be presented during each part of the activity?". After the analyses in small groups, the needs for all aspects were validated and refined in a plenary discussion and consensus was reached.

Workshop participants stated that grasping force was the most important type of information that should be fed back to the user, because it is impossible to derive this information through visual inspection. Applying the right amount of grasping force is essential when handling fragile objects or when interacting with humans and animals. Feedback about the position of the fingers was considered important to reduce the required visual attention and allow for more intuitive grasping. A combination of both grasping force and position information could provide the user with a measure of object 
stiffness. Feedback about the status of the control system, such as when grasp closure has been completed was also mentioned as being useful.

The discussion on feedback methods revolved mostly around the choice between continuous and discrete feedback. Although continuous feedback can improve the user's ability to handle the prosthesis intuitively, the user's perception of a non-physiological signal may fade over time. In contrast, discrete feedback should not be so abrupt that it disturbs the user. This type of feedback could be useful for indicating the status of the control system, but was considered less important than continuous feedback.

Feedback was only considered of added value when it is intuitive and simple. Other mentioned requirements for feedback were that it should be unobtrusive to others and comfortable to the user. The ability to adjust feedback for individual patients was also considered essential. The functional requirements for feedback are summarized below:

1) Continuous and proportional feedback on grasping force should be provided

2) Position feedback should be provided to the user

3) The stimulation used for feedback should be intuitive and easily interpretable

4) Feedback should be unobtrusive to the user and others

5) Feedback should be adjustable

Based on these requirements a literature overview of the state-of-the-art of feedback for myoelectric forearm prostheses was provided:

In a large number of studies on forearm prosthesis research, force and position information is directed to the prosthesis itself (e.g., in automated slip control) [87, 161], but efforts have also been made to provide feedback directly to the user, which will be described below. A natural way to close the prosthesis control loop that also incorporates feedback is the use of extended physiological proprioception (EPP) as proposed by Simpson [134]. In body-powered prostheses, the EPP principle is applied via the direct relation between shoulder movements and prosthetic hand movements. However, the focus of this review is on myoelectric prostheses, and therefore, feedback applications of the EPP principle are not considered here.

\section{Feedback requirement 1: Force feedback}

The most natural way to directly close the loop between sensing and feedback would be the direct stimulation of the afferent sensory nerves, which is being investigated in several studies [50, 51, 57]. To avoid the invasive character of this solution, but still provide feedback by the same modality (e.g. force to force), many researchers use extended physiological taction (EPT), in which force measured by force sensors is transmitted to the user via force applied to the skin with the same amplitude. Small servomotors with a little bar attached to the shaft can provide touch feedback [8, 37]. Other small systems have been developed as well to provide touch feedback [94] or pressure feedback $[81,96,104,106]$. In an approach by Gillespie et al. the grasping force feedback was related to the torque applied to the elbow via an external device [59]. For 
lower limb prostheses a system providing pressure feedback through small balloons was evaluated [55].

The use of the same modality to provide force feedback is potentially more intuitive than the use of another modality such as vibrotactile or electrotactile stimulation. In comparison studies on pressure stimuli and vibrotactile stimulation to provide pressure feedback, the pressure stimuli outperformed the vibrotactile stimulation via coin motors in spatial discrimination performance [9] and errors in applied grasping force [106]. However, the stimulators used for EPT are still bulky and cannot be incorporated fully in the prosthesis cover.

Early applications of force feedback have mainly used electrotactile stimulation. Force levels were modulated either by amplitude, following a linear [16] or nonlinear relation [121], or by pulse rate $[124,127,131,149,162]$. Effects of feedback were mainly subjectively evaluated and showed positive results [16, 124, 127, 131]. The rare quantitative analyses showed increased performance in grasping tasks [121, 149], even when visual feedback was available [162]. However, electrotactile stimulation has several potential disadvantages, the most significant of which is the likelihood of painful stimulations. Since there have been several advancements in vibrotactile stimulation (e.g., the miniaturization of the stimulators), most recent research projects have abandoned electrotactile stimulation in favor of vibrotactile stimulation.

Force feedback systems using vibrotactile stimulation have been incorporated in the prosthetic hands of three previously mentioned projects: the MANUS hand [112], Cyberhand [40], and Fluidhand [117]. A distinction can be made between studies using a C2 tactor (miniature vibrotactile transducer) and smaller coin motors. The amplitude and frequency of a C2 tactor can be separately controlled and therefore force feedback can be provided via stimulation frequency [40], pulse frequency [28, 39], or amplitude [39, 137] modulation. The frequency of stimulation of the coin motors cannot be controlled independently from the amplitude of stimulation. Therefore, frequency modulation in combination with amplitude modulation is used to provide force feedback in a couple of studies $[113,116]$. Furthermore, an array of coin motors was used to provide grasping force feedback via position modulation [123].

Subjective evaluation through questionnaires showed positive experiences in comfort and utility [40], but feedback became disturbing when applied continuously [116]. Results of grasping force feedback were variable over the studies. Evaluation of grasping performance in one of the studies showed a reduction (15-77\%) in excessive grasping forces [116], while in other studies no significant differences in grasping performance could be found in comparison to the non-feedback situation [28, 40].

\section{Feedback requirement 2: Position feedback}

In comparison to the application of force feedback, feedback of position is more rarely described. Only one study on electrotactile hand aperture feedback was found [114, 115], in which a combination of feedback about grasping force (by varying the pulse 
width) and feedback about the level of hand aperture (by pulse rate modulation) through a single electrode was used. Evaluation showed that it was not possible to provide force and position feedback using the same electrode, but performance in distinguishing object sizes did increase with feedback.

Several studies are performed on proprioceptive feedback about the movement of the index finger and evaluated in targeted movements. Feedback about the movement of the finger was provided either through passive movement of the index finger [20,83] or through skin stretch feedback. The skin stretch feedback was also compared with vibrotactile stimulation via a C2 tactor and showed better performances in a cursor movement task $[15,155]$.

In a completely different approach, the phantom sensation phenomenon [93], in which sensations are felt in between two simultaneously activated stimulators with different intensities, was used. Feedback of the level of flexion and extension of the elbow was provided by this method. The performance of subjects in matching and reaching tasks was considerably improved and comparable to performance with a body-powered prosthesis.

Recently, a study on feedback about several hand configurations was performed. In this study an array of C2 tactors, placed around the waist, was used [30]. They showed good performances in discrimination of the configurations, but subjects needed a lot of time to recognize the rather complicated stimulation patterns.

\section{Feedback requirement 3: Interpretability and intuitiveness}

Although the interpretability and intuitiveness of feedback are not described specifically for prosthesis applications, they are influenced by both the perception of stimulus intensity and the perceived sensation, which has been described in psychophysical studies.

The perceived stimulus intensity $(\psi)$ is strongly related to the applied stimulus intensity $(\phi)$ and best described by an power function, $\psi=k \phi^{\beta}[79,95]$. The exponent of the function can vary greatly for different stimulus conditions and the power function is usually adjusted by taking into account the stimulus threshold $\left(\phi=S-S_{0}\right)$, where $S$ is the stimulus intensity and $S_{0}$ the stimulus threshold. For electrotactile stimulation the variation in the exponent $\beta$ is mainly caused by the interaction between stimulus duration and stimulus intensity $[13,145]$, and for vibrotactile stimulation this variation is mainly caused by the location of stimulation [76]. The perceived stimulus intensity is influenced by the amplitude of stimulation, the duration and number of bursts of the stimulation, the housing of the stimulator, the characteristics of the preceding stimulus, and the number of simultaneous stimuli [31].

The perceived sensations with vibrotactile stimulation are influenced by intensity, frequency and waveform of the stimulation, actuator size, and location of stimulation. Therefore, descriptions vary largely in literature [76, 132], from buzzing to sharp pain. Variations in perceived sensations with electrotactile stimuli are related to stimulus intensity, electrode characteristics, preparation of the skin, and the use of cathodic or anodic stimulation $[78,109,145]$. It was shown that sensations perceived by amputees 
do not differ from the sensations of nondisabled subjects for percutaneous stimulation [7].

\section{Feedback requirement 4: User comfort}

During the workshop, comfort was defined as a prosthesis not being obtrusive and not causing pain or skin problems. Therefore, auditory or visual feedback options were considered to be unsuitable for feedback in forearm prostheses. As mentioned earlier, a major problem with feedback through electrical stimulation is the risk of generating painful sensations. This risk is influenced by the skin contacts, skin condition, type of stimulation (cathodic/anodic), and the size of the electrode [78]. In a first study on the long term effects of vibrotactile stimulation, no adverse effects were found after 4 weeks of use [6]. However, no extensive studies have been performed on the effects of longterm vibrotactile or electrotactile stimulation.

\section{Feedback requirement 5: Adjustability of location and stimulus intensity}

The last feedback requirement stated that the feedback should be adjustable for each prosthesis user. Two aspects of sensory feedback that can be adjusted are the location of stimulation and the stimulus intensity. The ability to adjust the location of stimulation is influenced by the effects of the stimulation location on (1) the sensitivity of the subjects to vibrotactile stimuli, (2) the effects of the location on the localization performance (ability to indicate the stimulus location), and (3) the effects on the smallest detectable distance between stimulators (spatial acuity). The ability to adjust the stimulus intensity is largely influenced by the adaptation to the stimulus. All these effects of vibrotactile or electrotactile stimulation have been investigated in literature, but their implications for prosthesis applications are not known.

At the glabrous skin, the sensitivity for vibrotactile stimulation is highest with an optimum at $250 \mathrm{~Hz}$ (with a detection threshold of only several microns of skin indentation), but at the hairy skin, the sensitivity is lower and the maximum shifts to 200-220 Hz [31, 76, 92]. In a study by Cholewiak et al., detection thresholds (defined by the amplitude of stimulation) of vibrotactile stimulation were measured over the whole length of the forearm, which resulted in equal thresholds at all locations [35].

Localization performance is highly influenced by the location of stimulation. Stimulation near bony landmarks resulted in significantly better localization of the stimuli [35]. Localization performance is not only influenced by the location of stimulation, but also by the space between the stimulators.

The spatial acuity highly depends on the stimulus location and can therefore vary greatly, from $2 \mathrm{~mm}$ at the finger tips to several centimeters at the back [95]. For electrotactile stimulation, variations can also be caused by changes in frequency, pulse width, and pulse time delays [135]. Furthermore, temperature and stimulus type affect the spatial acuity for both types of stimulation [31, 99]. A very clear decrease in spatial acuity was found for elderly subjects (65+) compared to youngsters (18-28 yrs.) [142]. However, the percentages of decrease were not the same for each body location. The strongest decrease in spatial acuity with aging was found for the toes (400\%), while at 
the fingertip this decrease was only $130 \%$. Not only the body location, but also the orientation of the stimulation array influences the spatial acuity. The orientation of the receptive fields of the main mechanoreceptors is anisotropic with larger fields in the longitudinal direction. Therefore, the spatial acuity for tactile stimuli is better for a transversal oriented (around the forearm) array in comparison to a longitudinal (from elbow to wrist) array [41].

The adjustability of the stimulus intensity becomes important when adaptation of stimuli occurs. Due to adaptation the perceived stimulus intensity decreases with time. To prevent this, the stimulus intensity should be adjusted. Clear adaptation curves (decreases in perceived intensities with continuous stimulation) were found for vibrotactile stimulation [67]. Adaptation with vibrotactile stimuli may not occur until after 25 minutes, while with pressure stimuli, adaptation can already occur after $1 / 2$ minute. Adaptation can be reduced by changing the frequency of the subsequent stimulus or by applying the stimuli intermittently [31, 130]. For electrotactile stimulation, adaptation is lowest for high current stimulation (just below the pain threshold) and can also be reduced by intermittent stimulation [26].

\section{Contents of the thesis}

It can be concluded that the need for sensory feedback in myoelectric forearm prostheses is clear, which is also confirmed by the increasing number of projects on this topic. However, essential knowledge is still missing. Especially there is a lack of knowledge on hand aperture feedback and evaluation of the optimal stimulation parameters to provide the feedback. Based on the literature review and the requirements following from the workshop, the focus of this thesis will be on feedback about hand aperture and grasping force through vibrotactile or electrotactile stimulation.

The contents of this thesis can be divided into three main parts: (1) investigation of basic features of (vibrotactile) feedback, (2) an experimental part, describing studies to derive optimal stimulation parameters, and (3) a clinically oriented part, describing studies evaluating the usefulness of the feedback.

\section{Part one}

This part describes the basic knowledge on which the other parts of the thesis are based. The requirements for feedback in myoelectric forearm prostheses are already described in the introduction of this thesis. An analysis of the possibilities of a single coin motor to provide vibrotactile feedback, based on mechanical and psychophysical characteristics, will be presented in chapter 1.

\section{Part two}

Hand aperture feedback will be described in chapter 3 and 4. First, a comparison of electrotactile and vibrotactile stimulation and a comparison of a longitudinal and transversal oriented stimulator array will be described (chapter 2), followed by an 
evaluation of the effect of distractive tasks on the feedback interpretation when performing grasping tasks (chapter 3). The search for optimal stimulation parameters to provide grasping force (and slip) feedback will be described in chapter 4 . Three modulation techniques (amplitude, frequency and position modulation) will be compared. In chapter 5 the combination of vibrotactile hand aperture and grasping force feedback in one system will be described and the performance of this system will be compared for several feedback configurations. A further extension is the combination of hand aperture and grasping force feedback during object holding, which will likely provide information about the stiffness of an object. The evaluation of the performance in object discrimination with stiffness feedback will be described in chapter 6 .

\section{Part three}

The experiments described in chapter 3 to 7 are all performed with a virtual setup controlled by mouse scrolling (with a scroll wheel), while the ultimate applications will be controlled by EMG. In chapter 7 the performance in grasping tasks will be compared between mouse scroll control and EMG control, evaluating the possible influence of the EMG control on the interpretability of the vibrotactile feedback.

The validation of the results of chapter 3 and 5 on subjects with upper limb loss, the ultimate users of the feedback, will be described in chapter 8.

Finally, in chapter 9 the results of the previous studies will be validated in daily life grasping tasks performed by healthy subjects using a myoelectric forearm prosthesis.

In the general discussion the main findings from the previous chapters will be discussed and recommendations for further research will be provided. Lastly, the main implications for myoelectric forearm prosthesis research of this thesis will be summarized. 


$$
\begin{aligned}
& \overline{5} \\
& + \\
& 0 \\
& 0 \\
& \frac{0}{1} \\
& \frac{7}{1} \\
& \text { o. }
\end{aligned}
$$

Introduction 
Chapter 1 - Vibrotactile stimulation with a single coin motor

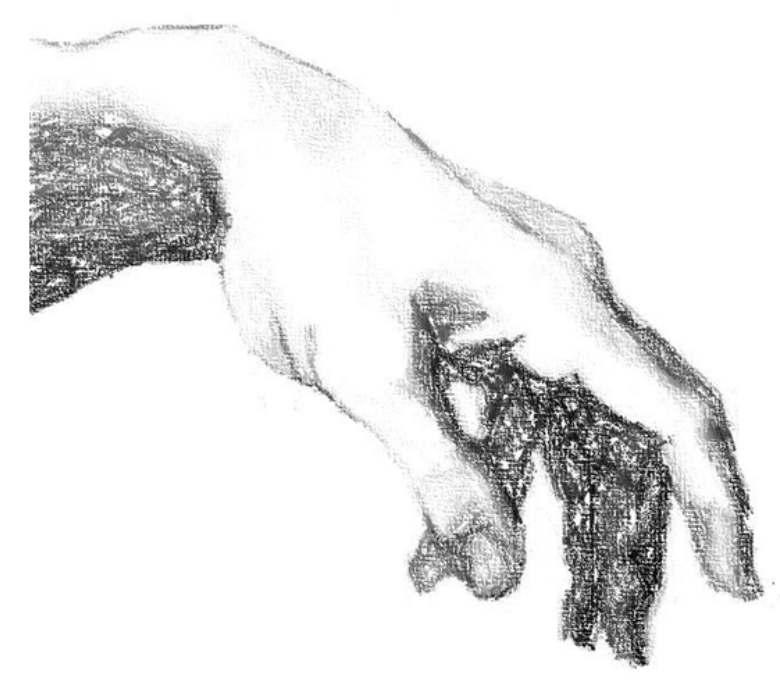

Psychophysical and mechanical characteristics of vibrotactile stimulation with a single coin motor at three forearm locations

Authors: Heidi Witteveen, Hans Rietman, Peter Veltink 


\section{Abstract}

Sensory feedback to the user is essential for optimal control of forearm prostheses, but lacking in current myoelectric prostheses. Vibrotactile stimulation can be used to provide this feedback in a comfortable and non-obtrusive way. The use of a small coin motor has been proposed to provide this feedback, but the psychophysical and mechanical characteristics are not known. Frequency control of stimulation and the measurement of displacements during stimulation is achieved by mounting an accelerometer on top of the coin motor. Mechanical characteristics of the coin motor stimulation in combination with the underlying skin of ten healthy subjects were investigated, as well as psychophysical characteristics (magnitude estimation) during varying stimulation frequencies. Both characteristics were determined at three locations of the arm. It is shown that the mechanical characteristics of the system differ significantly over the three locations, indicating the need for adaptable stimulation methods, but no differences were found for the psychophysical characteristics. Sensory feedback through stimulation of a single coin motor was comfortable and easily applicable, but a limited number of stimulation levels could be distinguished and therefore it is recommended to use an array of coin motors. 


\section{Introduction}

Amputation or a congenital defect of the forearm causes a high level of disability for the people concerned. A prosthesis should be able to take over the function of the missing hand, but in practice the mechanism of the human hand is very complex and cannot even be approached by a prosthesis. The development of externally powered (myoelectric) prostheses has improved the more natural control of the prosthesis, but does generally not provide adequate non-visual feedback [110].

This lack of feedback in myoelectric prostheses causes the user to almost fully rely on visual and to some extent auditory information and haptic information about external loads. The continuous use of the visual sensory system to control the prosthesis causes a high mental burden, a poor integration of the prosthesis with the human body, and does not allow subconscious control of the prosthesis [143]. Results of extensive surveys under prosthesis users showed that one of the main improvements for current prostheses would be the development of a prosthesis requiring less visual attention [12] and according to this, sensory feedback was indicated, by $88 \%$ of enquired health care professionals, as being important in upper-limb prostheses [113].

Artificial feedback can be applied by using the visual, auditory, proprioceptive or tactile sensory system. The use of tactile feedback is preferred over visual or auditory feedback, because tactile cues are less disruptive and the sense of touch is relatively underused compared to the visual and auditory modalities and therefore the chance of sensory overload is rather small [6,115, 128]. These aspects also hold for proprioceptive feedback, but the practical application of this kind of feedback is more complicated. One of the major disadvantages of electrotactile stimulation is the limited number of possible stimulation amplitudes between the sensation and the pain thresholds [76-78]. Therefore, vibrotactile stimulation is nowadays preferred over electrotactile stimulation, which is also due the recent miniaturization of the vibrotactile stimulators.

Recent studies on vibrotactile grasping force feedback often used the commercial C2 tactor (Engineering Acoustics) [28, 39, 40, 140, 141], which is a linear vibrotactile transducer, moving perpendicular to the skin. The resonance frequency of the C2 tactor is 250 Hertz, which is the frequency at which the Pacinian mechanoreceptors are most sensitive [79]. However, The $\mathrm{C} 2$ tactor is still quite bulky $(3 \mathrm{~cm}$ diameter and $0.5 \mathrm{~cm}$ height) and therefore difficult to apply in upper-limb prostheses.

A single coin motor ( $1 \mathrm{~cm}$ diameter vibrating element, used in mobile phones) has also been proposed to provide (grasping force) feedback [116]. This coin motor consists of a rotating mass, moving in line with the skin and thereby providing tangential stimulation. Furthermore, the stimulation frequency of these coin motors is lower in comparison to the $\mathrm{C} 2$ tactor and therefore other mechanoreceptors may be activated. A main drawback of these stimulators is the direct coupling between an increase in frequency and an increase in the amplitude of movement, while in most linear vibrotactile transducers, these quantities can be controlled independently. 
It is not clear how useful the coin motors are in providing sensory feedback, because the psychophysical and mechanical characteristics of this kind of vibrotactile stimulation have not been investigated before.

Evaluation of mechanical characteristics of the skin during (vibro) tactile stimulation at different stimulus locations has been rarely described in literature. Some examples of impedance measurements during vibrotactile stimulation at a single body location can be found $[63,80,91,98]$. In these cases, stimulation was provided perpendicularly to the skin of the fingers or hand, except for Moore et al. [98] who also performed experiments on the arm. The implications of the results of these studies for feedback applications have not been described.

Psychophysical characteristics of vibrotactile stimulation are described in literature more often $[35,92,148]$, but mostly in relation to the glabrous skin. Some of them [35, 92] have also investigated different locations of stimulation and their effects on the psychophysical parameters, but used large vibration motors that cannot be applied in forearm prostheses. Furthermore, they did not combine their results with the mechanical characteristics of the skin, while differences in mechanical characteristics of the skin could possibly influence the perception of the stimuli.

In this study both mechanical and psychophysical characteristics of coin motor stimulation were evaluated at three locations on the forearm to determine the effect of mechanical characteristics on the psychophysical characteristics (the stimulus perception) and to evaluate the possibilities of providing feedback through a single coin motor.

\section{Methods}

\section{Subjects}

Measurements were performed on 10 healthy subjects (students, 21-27 years). All subjects were informed about the research preceding the experiment and provided informed consent. The stimulators were attached to the non-dominant arm of the subject by double-sided tape, because the dominant arm was used to control the mouse to give responses after stimulation.

During the experiment, subjects were seated comfortably with their arm resting on the table in such way that they could easily read the screen of the laptop placed in front of them, were able to control the mouse and could stay in this position for at least half an hour without moving their arm.

\section{Coin motor characteristics}

A commercially available coin motor (Coin Motor, iNeed, China, see Figure 1a) was used, which consists of a cylindrical, flat housing, with an asymmetrically positioned rotating mass inside. When placed horizontally on the skin, the skin is stimulated tangentially. The coin motor is current driven and by increasing the current, the frequency of rotation increases, which results in an increased force. The relationship between the applied current and the frequency of vibration is influenced by the characteristics of the load to 
which it is attached. To enable the control of stimulation frequency, a small 3-axial accelerometer (MMA7341L, Freescale Semiconductor Inc., Tempe, Arizona) was glued on top of the coin motor (see Figure 1a). The power spectrum of the accelerometer data was calculated over a sliding window of $100 \mathrm{msec}$. and the frequency of vibration was calculated as the frequency with the highest amplitude in this spectrum. A proportional and integrative (PI) controller was used to adjust the motor current with respect to the difference between set and measured frequency. This PI controller was implemented in a Labview routine, which controlled the current supply from the computer to the coin motor. Every $50 \mathrm{msec}$. the measured frequency is compared to the set frequency and the controlling current is adjusted by the PI controller. Prior to the start of the experiments, the PI-controller gains were tuned manually to create responses that were acceptable, with lowest settling times (the time between the onset of the stimulation and the point where the set frequency was reached), least overshoot (maximal difference between the measured and set frequency) and no oscillation, for the whole range of stimulation frequencies. The frequency control of the coin motor allowed the evaluation of the mechanical characteristics of the skin as well as the psychophysical aspects measured at different stimulation frequencies.

The relationship between the applied current and resulting frequency likely depends on the mechanical characteristics of the skin to which it is attached. Therefore, the range of stimulation is expected to vary over the stimulation locations and subjects.

(a)

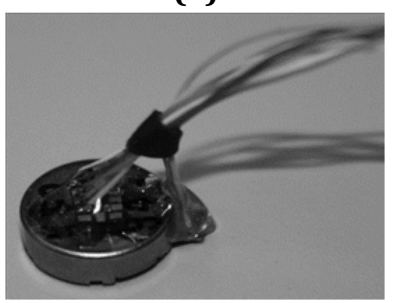

(b)

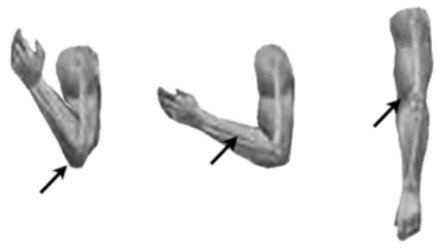

Figure 1: (a) Coin motor used to provide the vibrotactile stimulation with an accelerometer mounted on top to control the frequency and measure the amplitude of stimulation. (b) The three stimulation locations from left to right: the dorsal side of the elbow, the dorsal side of the forearm and the ventral side of the elbow

\section{Measurement locations}

Measurements were performed at three locations on the arm. The coin motor was placed (1) on the dorsal side of the elbow on a relatively flat surface just distal to the olecranon process, (2) at the dorsal side of the forearm, midway between the elbow and the wrist and (3) on the ventral side of the elbow, above the tendon of the biceps (see Figure 1b). The skin was stretched during the experiments; for the measurements at the ventral and dorsal sides of the elbow, the elbow was maximally extended respectively flexed. The three stimulation locations were not considered possible or ideal locations for feedback stimulation in upper-limb prostheses, but were selected because the underlying structures were very different. Stimulators placed at the ventral side of the elbow are placed close to underlying tendons, while bone is closer to the surface at the 
dorsal side of the elbow. The order of trials at the three locations was randomly selected per subject. During the experiments, the subjects wore headphones that presented white noise to eliminate auditory cues from the coin motor. The experimental procedure was automated to shorten the duration of the measurements. Instructions were given verbally before each trial.

\section{Mechanical characteristics of the skin}

Mechanical characteristics of the skin were derived via calculation of the displacements of the coin motor during stimulation at different frequencies. For this purpose, stimulation was performed at frequency intervals of $5 \mathrm{~Hz}$, three times per interval. Accelerometer data in a direction parallel to the skin was integrated twice to derive the displacements of the coin motor during stimulation. The maximum displacement was calculated as the peak amplitude of the displacement signal between 1000 and 1500 msec. from the start of the stimulation. Displacements were averaged over the three subsequent measurements per frequency level. These measurements also determined the stimulation range. Frequency levels were considered outside the stimulation range when the error between the required and actual frequency was larger than $2 \mathrm{~Hz}$ over the whole stimulation duration.

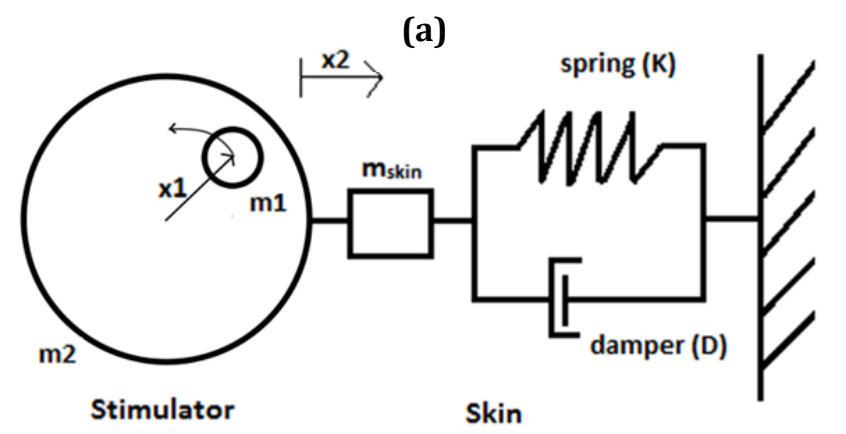

(b)

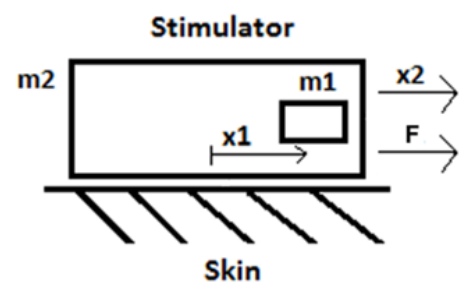

Figure 2: Model representation of the coin motor attached to the skin. (a) model of the second order system and (b) representation of the orientation of the coin motor to the skin

The human skin was modeled as a second order system, consisting of a mass (the coin motor and an effective mass component of the skin) connected to a spring and damper (representing the stiffness and damping of the skin) as shown in Figure 2. The force exerted by the coin motor is generated by the acceleration $a_{m 1}$ of the rotating inner mass $m_{1}$ :

$$
F=m_{1} * a_{m 1}=m_{1} * x_{1} * \omega^{2} *(-\sin \omega t),
$$

where $x_{1}$ is the radius of rotation and $\omega$ the angular velocity. The mass of the rotating part of the stimulator, $m 1$, was determined by an accurate analytical balance (MettlerToledo A9245, Zürich, Switzerland) to be 0.32 grams. The total mass of the coin motor was 1.08 grams. During preliminary measurements the force was measured at distinct stimulation frequencies between 40 and $110 \mathrm{~Hz}$ through an accurate force sensor (custom made strain gauge force transducer) on which the coin motor was glued in a vertical position. A second order polynomial curve was fitted on the force data and in 
combination with the known parameters, the radius of rotation $\left(\mathrm{x}_{1}\right)$ was calculated from the derived coefficient of the polynomial. The estimated value for $\mathrm{x}_{1}$ was $2.7 \mathrm{~mm}$.

For the main experiments, the force exerted by the coin motor was calculated for each frequency level, based on the known values for the mass $\left(\mathrm{m}_{1}\right)$ and radius $\left(\mathrm{x}_{1}\right)$, and used as input for the model. The output of the system is the displacement of the coin motor, $x_{2}$. The corresponding transfer function of the second order system, relating the displacements of the coin motor to the applied force, is:

$$
y_{m o d}(\omega)=\frac{x_{2}(\omega)}{F(\omega)}=\frac{1}{m j \omega^{2}+D j \omega+K}
$$

where $D$ and $K$ are the parameters related to the damping and stiffness of the system respectively. The coefficient $m$ is the mass constant for the whole system (the coin motor and the effective mass of the skin) and therefore differs from the mass $m_{1}$ of the rotating part. The system parameters were derived for every measurement by fitting this second order model to the measurement data for each subject and location of stimulation separately. An error function, describing the summed quadratic error between the model $y_{\text {mod }}$ (described by the transfer function) and the data points $y_{i}$ (measured displacements divided by the calculated forces) for each stimulation frequency, was formulated:

$$
\varepsilon=\sum_{i=1}^{n}\left(\left|y_{\bmod _{i}}\right|-\left|y_{i}\right|\right)^{2}
$$

The Matlab function fminsearch was used to find the values for the constants $m, D$ and $K$ that minimized this error function. From the values for the mass, stiffness and damping, the natural frequency $\left(\omega_{n}=\sqrt{\frac{K}{m}}\right)$ and damping ratio $\left(\zeta=\frac{D}{2 * \sqrt{K * m}}\right)$ were also derived for every measurement. A damping ratio below 1 corresponds to an underdamped system, a value of 1 to a critically damped system and a value of above 1 to an overdamped system [103].

\section{Psychophysical characteristics}

The subjective intensity perception of single stimuli was evaluated by the psychophysical method of magnitude estimation [58]: a stimulus with a selected frequency and duration of 2 seconds was presented after which the subject was asked to indicate the perceived intensity on a scale. The subjects were free to choose the range of the scale (no fixed limits). Frequency levels were selected from the earlier derived range of stimulation at intervals of $10 \mathrm{~Hz}$, starting from the lower limit $+5 \mathrm{~Hz}$. The stimulation range could vary over locations and subjects. Each trial consisted of 10 stimuli per frequency level and therefore the total number of stimuli in a trial could vary as well.

Preceding statistical analysis of the perceived intensities, the data was tested for normality by evaluation of the skewness and kurtosis values and visual inspection of the Q-Q plots (quantiles of the dataset plotted against normal theoretical quantiles). The 
perceived intensities of the stimuli were first evaluated via a one-way ANOVA analysis to determine if the perceived stimulus intensities were significantly influenced by the stimulation frequency. Afterwards, Bonferroni post-hoc t-tests were performed to determine if the mean perceived intensity at one frequency level differed (with a significance level $\mathrm{p}=0.05$ ) from the perceived intensity at another frequency level. These t-tests were performed for every combination of frequency levels within the stimulation range per subject. When it was known which frequency levels could be distinguished from each other within the stimulation range, the total number of distinguishable frequency levels and the difference between distinguishable frequency levels (in $\mathrm{Hz}$ ) were derived. Furthermore, the ratio between the number of distinguishable levels and the total number of frequency levels within the stimulation range was calculated, which allowed the comparison between subjects and locations with a different stimulation range. All parameters were averaged over all subjects.

Finally, the subjects were asked to score the level of perceived comfort of the stimulation on a Visual Analog Scale (VAS), ranging from very uncomfortable to very comfortable (10 cm line on paper). The VAS was scored after completion of a trial for each stimulation location.

\section{Results}

\section{Stimulator characteristics}

The mean settling times was 744 msec. ( \pm 282 msec.), which is clearly within the duration of the stimulation. The overshoot at the lowest stimulation frequencies was $19.92 \pm 4.2 \mathrm{~Hz}$ and at the highest stimulation frequencies the overshoot was lower $(5.13$ \pm 6.6 . Hz). No clear differences in settling times and overshoot were seen for the different stimulation locations.

\section{Mechanical characteristics}

Although not all measurements covered the same stimulation range $( \pm 30-100 \mathrm{~Hz})$, all measurement data was used to create a figure showing the mean and corresponding 95\% confidence intervals of the displacements aggregated over all 10 subjects at the three different stimulation locations (see Figure 3a). 
(a)

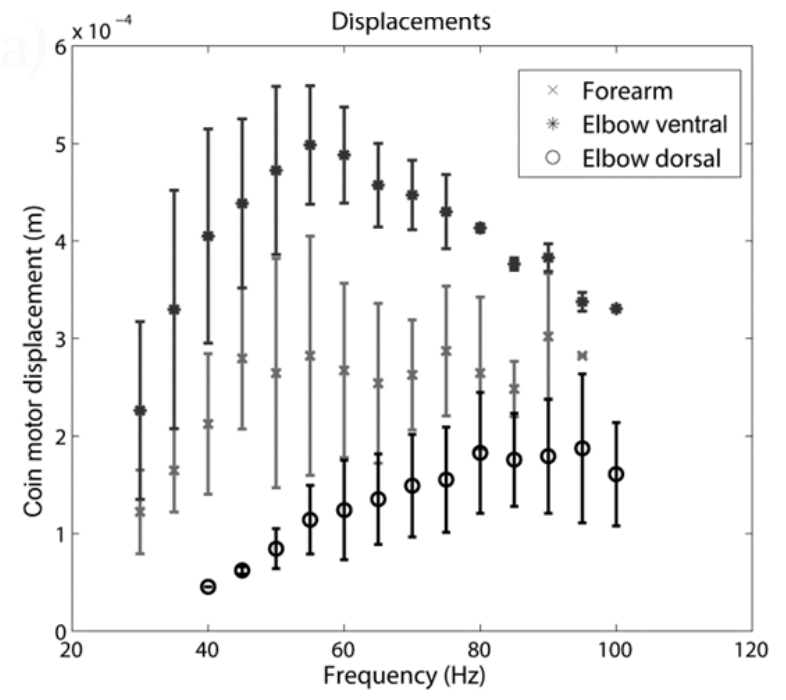

(b)

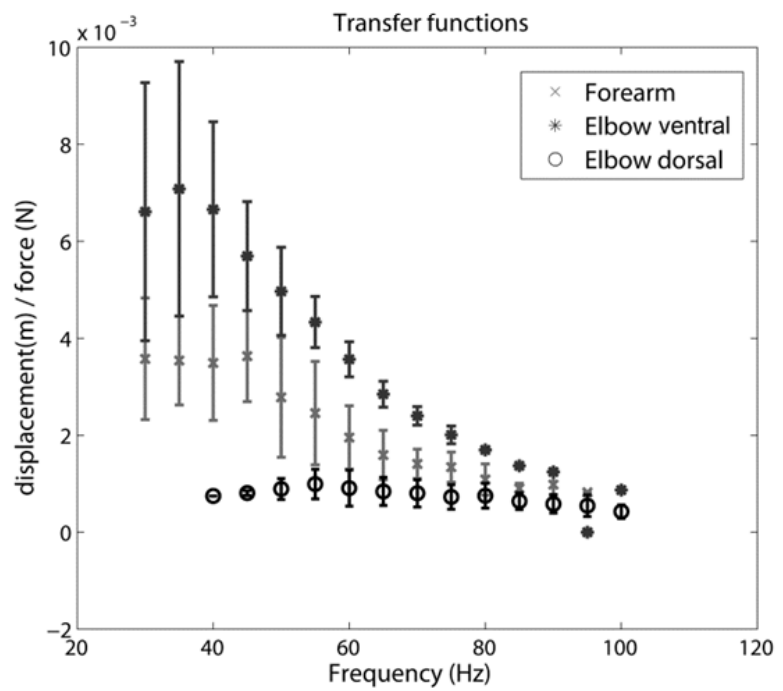

Figure 3: Mean and $95 \%$ confidence intervals of (a) displacements and (b) transfer functions of all 10 subjects at the three locations.

From the figure it can be seen that the amplitudes of vibration differ per location of stimulation. Moreover, it shows that especially for the data measured at the ventral side of the elbow, the amplitude of displacement decreases beyond a certain frequency (around 50-65 Hz) within the stimulation range. Corresponding transfer plots (see Figure $3 \mathrm{~b}$ ) show the second order behavior for the measurements at the forearm and the ventral side of the elbow.

For every measurement the mass, stiffness and damping constants were derived from the data fitting and based on these values the damping ratio and natural frequency were calculated (see Table 1). For some measurements at the dorsal side of the elbow $(n=4)$, the stimulation range was not large enough to cover the low-frequency behavior of the system and fitting with the mass-spring-damper system was not possible. Therefore, the only parameter that was fitted on this data was the mass constant (last row Table 1). It can be seen that the frequency range for this stimulation at the dorsal side of the elbow is shifted more to the higher frequencies compared to the other locations.

All values for the mechanical characteristics were higher for the measurements at the dorsal side of the elbow compared to the other locations. No statistical analysis was performed on the data, because stiffness and damping for the dorsal side of the elbow could not be derived for every subject and the number of measurements was variable over the different stimulation locations. 
Table 1: System parameters derived at the three measurement locations ( $m=$ mass, $D=$ damping, $\mathrm{K}=$ stiffness). Natural frequency $(\omega \mathrm{\omega n})$ and damping ratio $(\zeta)$ are derived from these parameters and the quality of the fit is expressed in R2 values. Mass parameters only are derived in four cases at the dorsal side of the elbow (could not be fitted by mass-spring-damper system).

\begin{tabular}{|c|c|c|c|c|c|c|c|}
\hline & $\begin{array}{c}\text { Frequen } \\
\text { cy range } \\
\text { (Hz) }\end{array}$ & m (kg) & $\begin{array}{l}D(N \\
s / m)\end{array}$ & $K(N / m)$ & $\omega_{n}(\mathrm{~Hz})$ & $\zeta$ & $\begin{array}{l}R^{2} \text {-value } \\
\text { of the fit }\end{array}$ \\
\hline $\begin{array}{l}\text { Forearm } \\
\text { Mean (SD) } \\
(\mathrm{n}=10)\end{array}$ & $\begin{array}{c}36(13) \\
- \\
81(9)\end{array}$ & $\begin{array}{c}0.18 \\
(0.06)\end{array}$ & $\begin{array}{c}6.39 \\
(2.59)\end{array}$ & $\begin{array}{c}448.63 \\
(260.16)\end{array}$ & $\begin{array}{c}52.01 \\
(12.19)\end{array}$ & $\begin{array}{c}0.37 \\
(0.23)\end{array}$ & $\begin{array}{c}0.79 \\
(0.27)\end{array}$ \\
\hline $\begin{array}{l}\text { Elbow ventral } \\
\text { Mean (SD) } \\
(n=10)\end{array}$ & $\begin{array}{c}37(16) \\
- \\
82(13)\end{array}$ & $\begin{array}{c}0.12 \\
(0.05)\end{array}$ & $\begin{array}{c}3.6 \\
(0.72)\end{array}$ & $\begin{array}{c}368.50 \\
(304.62)\end{array}$ & $\begin{array}{c}50.30 \\
(10.74)\end{array}$ & $\begin{array}{c}0.36 \\
(0.17)\end{array}$ & $\begin{array}{c}0.84 \\
(0.27)\end{array}$ \\
\hline $\begin{array}{l}\text { Elbow dorsal } \\
\text { Mean (SD) } \\
(\mathrm{n}=6)\end{array}$ & $\begin{array}{c}52(13 \\
- \\
103(18)\end{array}$ & $\begin{array}{c}0.43 \\
(0.24)\end{array}$ & $\begin{array}{l}14.83 \\
(6.63)\end{array}$ & $\begin{array}{c}1140.40 \\
(581.88)\end{array}$ & $\begin{array}{c}74.97 \\
(21.84)\end{array}$ & $\begin{array}{c}0.40 \\
(0.31)\end{array}$ & $\begin{array}{c}0.83 \\
(0.21)\end{array}$ \\
\hline $\begin{array}{l}\text { (only mass } \\
\text { system, } n=4 \text { ) }\end{array}$ & $\begin{array}{c}75(23) \\
- \\
100(15)\end{array}$ & $\begin{array}{c}0.24 \\
(0.16)\end{array}$ & $\mathrm{n} / \mathrm{a}$ & $\mathrm{n} / \mathrm{a}$ & $\mathrm{n} / \mathrm{a}$ & $\mathrm{n} / \mathrm{a}$ & $\begin{array}{c}0.69 \\
(0.46)\end{array}$ \\
\hline
\end{tabular}

\section{Psychophysical characteristics}

The data of the single intensity perception showed values of skewness and kurtosis all between -2 and 2 and the data points the Q-Q plots are distributed around a straight line, which indicated normality of the data and therefore allowed the application of ANOVA for further analysis. ANOVA tests showed that for every stimulation location and every subject, the perceived stimulus intensity was significantly influenced by the stimulation frequency ( $\mathrm{p}$-values <0.05). Afterwards, t-tests were performed for every combination of two frequency levels within the stimulation range for every stimulation location and subject. Frequency levels were marked as distinguishable if $\mathrm{p}<0.05$. On average, only two frequency levels, with intervals between 19 to $26 \mathrm{~Hz}$, could be distinguished within the stimulation range (see Table 2). In addition, the ratio between distinguishable levels and stimulation levels and the averaged differences (in $\mathrm{Hz}$ ) between distinguishable levels were calculated (Table 2). Furthermore, an example result plot is given (see Figure 4) for a specific subject and stimulation location.

Table 2: Ability to distinguish intensity levels at the three measurement locations

\begin{tabular}{|l|c|c|c|}
\hline & $\begin{array}{c}\text { Forearm } \\
\text { mean (SD) }\end{array}$ & $\begin{array}{c}\text { Elbow ventral } \\
\text { mean (SD) }\end{array}$ & $\begin{array}{c}\text { Elbow dorsal } \\
\text { mean (SD) }\end{array}$ \\
\hline \# levels & $4.1( \pm 0.9)$ & $4.3( \pm 2.3)$ & $4.9( \pm 1.6)$ \\
\hline $\begin{array}{l}\# \quad \text { levels that could be } \\
\text { distinguished (95\% c.i.) }\end{array}$ & $2( \pm 0.9)$ & $1.6( \pm 1.2)$ & $1.9( \pm 0.9)$ \\
\hline $\begin{array}{l}\text { Ratio of distinguishable levels } \\
\text { and total \# levels }\end{array}$ & $0.48( \pm 0.2)$ & $0.38( \pm 0.3)$ & $0.44( \pm 0.2)$ \\
\hline Difference between levels (Hz) & $19( \pm 6)$ & $26( \pm 9)$ & $23( \pm 14)$ \\
\hline
\end{tabular}




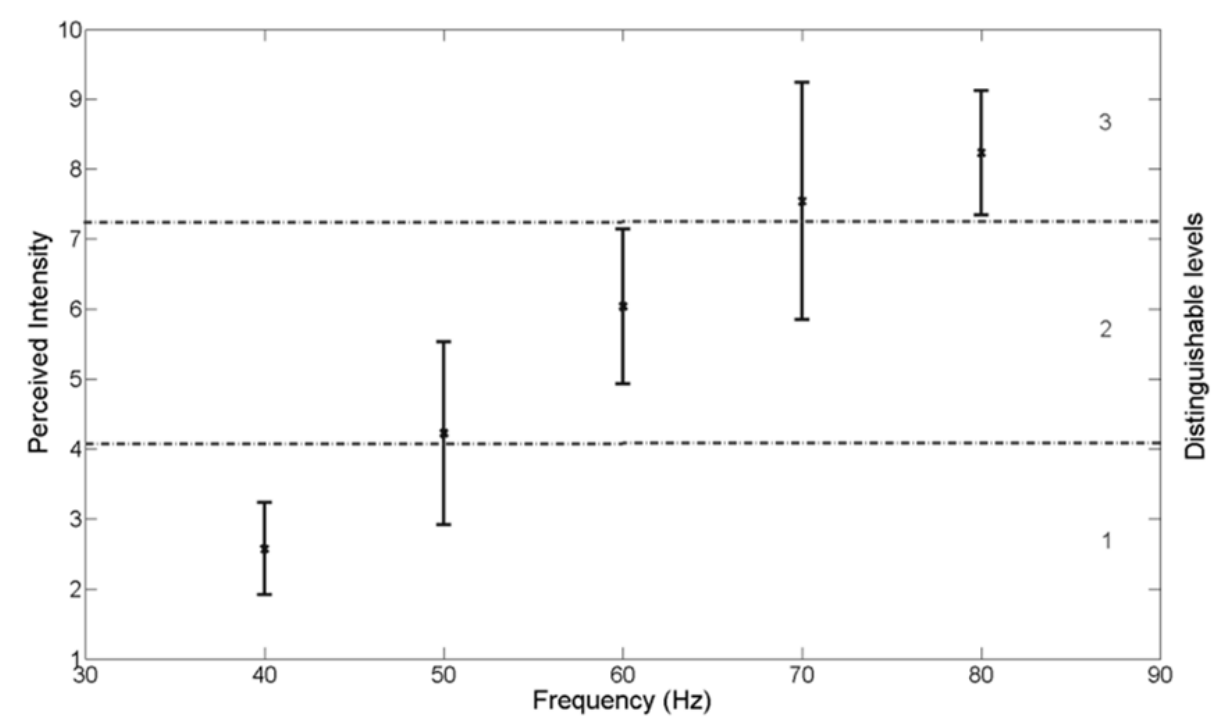

Figure 4: Typical example of mean perceived intensities and $95 \%$ confidence intervals at different frequency levels measured at the outer side of the elbow. The number of levels is 5 , the number of distinguishable levels is 3 , the ratio is 0.6 and the difference between distinguishable levels is 20 Hz.

Finally, the mean VAS scores for comfort of stimulation per location were calculated. Mean scores for stimulation at the forearm, the ventral and dorsal side of the elbow are $6.88( \pm 1,12), 6.24( \pm 1.85)$ and $5.95( \pm 1.92)$ respectively. All three locations were reported by the subjects as being rather comfortable and no significant differences between the VAS scores were found ( $p>0.05)$.

\section{Discussion}

\section{Sensory feedback via coin motors}

Commercial coin motors have been proposed to provide sensory feedback, because of their low costs and small size. In this study the possibilities of vibrotactile stimulation through a single coin motor have been investigated by evaluation of psychophysical and mechanical characteristics during frequency controlled stimulation. Stimulation characteristics showed that it is possible to control the frequency of stimulation by a PIcontroller. Within a duration of $750 \mathrm{msec}$., the frequency of stimulation steadily equals the set frequency. Stimulation at higher frequencies resulted in the lowest settling times (time between onset and steady stimulation), which should be kept in mind when a constant stimulation frequency will be used. For dynamic and rapid feedback applications these settling times are not very fast and less suitable, but the change in frequency starts immediately and may be noticed relatively quickly. Part of the settling time is also covered by the discrete character of the PI-controller, which is updated every 50 msec., and the length of the sliding window over which the frequency is calculated. Both aspects likely can be improved in future developments, but have not caused any problems in these experiments. 


\section{Mechanical characteristics}

Evaluation of the coin motor displacements (see Figure 3), derived from the measurements at the three different stimulation locations, showed clear differences between these locations. For measurements at the ventral side of the elbow the amplitude of vibration clearly decreases with increasing frequency (beyond frequencies of 50-65 Hz). As a result, some frequency levels within the stimulation range occurred with the same amplitude of vibration, which means that for this location the frequency is the only factor to distinguish intensity levels and likely diminishes the stimulus interpretation. For vibrations at the dorsal side of the elbow, there is a small, but clear increase in amplitude with increasing frequency over the whole range of stimulation. In literature it was shown that an increase in intensity in combination with an increase in frequency enhances the sensitivity $[36,100]$. According to this, it could be expected that the results of the psychophysical experiments would be superior for the dorsal side of the elbow, which however could not be confirmed by this study.

Impedance measurements with vibrotactile stimulation at the arm described in literature have mainly focused on stimulation perpendicular to the skin. However Diller et al. also conducted impedance measurements at the arm during tangential stimulation via a large stimulation apparatus [52]. They derived impedances, at the forearm, wrist and fingerpad, by keeping the amplitude constant and measuring the force at the tip of the stimulator. Their results showed large differences in impedance plots between subjects, which was also seen in our results, where standard deviations in skin parameters values are large. According to this, the implication for future tactile displays would be that they should be adaptable to a large range of stimulation (frequency and amplitude) to be applicable for all possible skin conditions.

The locations of stimulation used in this experiment were selected to create three clearly different locations and were not based on their possibilities for application in prostheses. When choosing a stimulation location for application in a prosthesis, it should be noted that the mechanical skin characteristics can vary largely over positions as well as over subjects as shown by this study. The input (force), used to create the transfer plots, could not be measured simultaneously with the output (displacements), but was calculated in advance from the mass (m1), the frequency of the coin motor and the derived radius of rotation ( $\mathrm{x} 1$ ). The determination of the radius of rotation through curve fitting could have induced some inaccuracy, but this did not influence the relative values of the system parameters, because possible errors in the input were the same for every measurement.

The determination of the mechanical characteristics by fitting the data by a second order transfer function is confirmed by the relatively high correlation coefficients that were achieved. However, some data could not be fitted by the mass-spring-damper system, because of the limited stimulation range. Therefore, only the mass parameter could be derived, which made comparison by repeated measures analysis over stimulation locations impossible. 


\section{Psychophysical characteristics}

No differences in the ability of subjects to distinguish frequency levels were found for the different locations of stimulation. Mahns et al. [92] concluded from their results that the decoding of frequencies is the same at different locations. In our study, the change in frequencies is also related to an increase in amplitude (except for stimulation at the ventral side of the elbow), which still not resulted in significant differences. The distribution of mechanoreceptors over the body is highly correlated with the cortical representation of the body parts [133], resulting in a high density of mechanoreceptors at the extremities, which decreases from distal to proximal. A larger density is related to a higher sensitivity and therefore it can be expected that the sensitivity will be larger at the forearm compared to the elbow, resulting in a larger number of distinguishable levels. However, it is also assumed that the density of mechanoreceptors (especially the Pacinian corpuscles) is large around bones and in ligaments [108], and those can probably be stimulated at the elbow sites. Whether these mechanoreceptors could be stimulated by the coin motors cannot be concluded from our results.

On average two frequency levels could be distinguished in this study. Weber fractions (differential threshold divided by the reference frequency, expressed in percentages) for frequency discrimination at the forearm of $2-50 \%$ can be found in literature [76]. These percentages would imply differential thresholds of 1.5 to $37.5 \mathrm{~Hz}$ for stimulations around $75 \mathrm{~Hz}$, resulting in 1-30 distinguishable levels for stimulation ranges of $45 \mathrm{~Hz}$. These differential thresholds were determined by using a reference stimulus from the same vibrator and it is commonly seen that the human body responds best to these kind of changes in stimuli, as for example shown in studies on nerve responses to stimulation [69]. No reference stimulus will be used in the ultimate application of the sensory feedback, and therefore the number of distinguishable levels with a single coin motor will be limited.

Magnitude estimation data is usually analyzed after normalization and log transformation of the data to compensate for the individual differences in scoring. However, this was not possible with our data, because the stimulation range varied over the subjects and therefore also the number of stimulation levels varied. To overcome these inter-subject differences, a ratio between the number of distinguishable levels and the number of available stimulation levels was determined.

\section{Future directions}

The subjects who participated in the experiments did not have any experience with vibrotactile stimulation. It is assumed that a period of training would increase the performance in the distinguishing tasks, which should be investigated further. However, feedback applications that require the least amount of training will probably be best accepted and most intuitive.

The measurements of this study and other studies on stimulus perception are all conducted on healthy subjects, but it cannot be assumed directly that the sensibility of the skin, the mechanical characteristics and the processing of stimuli are the same for 
healthy subjects and amputees. These aspects should be investigated in order to develop optimal feedback applications for prosthesis users.

\section{Conclusion}

Although differences in mechanical characteristics over stimulation locations were found, no differences were found for the psychophysical parameters investigated in this study, which implies a minor effect of the mechanical characteristics on the stimulus interpretation or the existence of a compensatory effect from for example the distribution of mechanoreceptors in the skin, which could not be concluded from our study. Based on the lack of differences in psychophysical parameters, no clear preference for one of the stimulation locations could be given and the choice for a stimulation location is therefore not restricted. However, differences in skin characteristics can occur between stimulation locations and large variability between the subjects was found, which shows the need for adaptable tactile displays.

The applicability of a single coin motor to provide for example grasping force feedback is questionable, because the number of stimulation levels that can be distinguished is limited. Furthermore, the settling time is too high to be useful for dynamic feedback applications. However, vibrotactile stimulation through a single coin motor has shown to be very comfortable to the user and is easily applicable. Therefore, it is recommended to use an array of more coin motors, using localization aspects, to provide sensory feedback. 


\section{Part 2}

Evaluation of stimulation parameters 
Chapter 2 - Vibro- and electrotactile user feedback on hand aperture

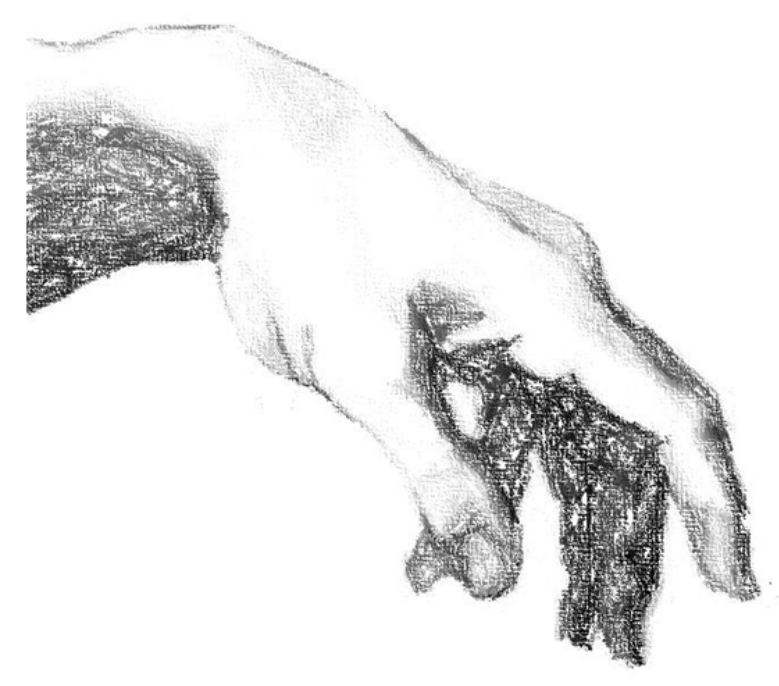

Published as: Vibro- and electrotactile user feedback on hand opening for myoelectric forearm prostheses

Authors: Heidi Witteveen, Ed Droog, Hans Rietman, Peter Veltink In: IEEE Transactions on BioMedical Engineering, vol. 59. no. 8, pp. 2219-2226, 2012 


\section{Abstract}

Many of the currently available myoelectric forearm prostheses stay unused because of the lack of sensory feedback. Vibrotactile and electrotactile stimulation have high potential to provide this feedback. In this study, performance of a grasping task is investigated for different hand aperture feedback conditions on 15 healthy subjects and validated on three patients. The opening of a virtual hand was controlled by a scroll wheel. Feedback about hand aperture was given via an array of eight vibrotactile or electrotactile stimulators placed on the forearm, relating to eight hand aperture positions. A longitudinal and transversal orientation of the array and four feedback conditions were investigated: no feedback, visual feedback, feedback through vibrotactile or electrotactile stimulation, and addition of an extra stimulator for touch feedback. No influence of array orientation was found for all outcome parameters (duration of the task, the percentage of correct hand apertures, the mean position error, and the percentage deviations up to one position). Vibrotactile stimulation enhances the performance compared to the non-feedback conditions. The addition of touch feedback further increases the performance, but at the cost of an increased duration. The same effects were found for the patient group, but the task duration was around $25 \%$ larger. 


\section{Introduction}

Despite the large improvements made in the development of myoelectric upper-limb prostheses, the number of prostheses that is not used on a regular basis remains quite high $(30-60 \%)[12,53]$. Several surveys have indicated that prostheses' abandonment is related to the poor functionality of the currently available prostheses and the lack of sensory feedback $[12,18,113]$. In a recent study, in which representative prosthesis users were involved, it is shown that feedback about the grasping force and the hand aperture are the most important aspects to be incorporated in future myoelectric forearm prostheses [107]. Feedback about the opening of the hand is especially important in situations where no visual feedback is available. Furthermore, a prosthesis with hand aperture feedback may reduce the amount of visual attention needed to control the prosthesis and thereby increase the acceptability of the prosthesis by the user. Several approaches to provide hand aperture feedback for upper-limb prostheses have been described. The use of phantom sensations to provide proprioceptive feedback was investigated at an early date [93]. For this approach, two vibrotactile stimulators, activated with different amplitudes to create (phantom) sensations in between the stimulators, were used to provide information about the elbow angle of an upper limb prosthesis. A direct connection to the afferent nerves was investigated by Dhillon and Horch who used implanted electrodes to provide information about the elbow range [50]. Electrostimulation of the skin was evaluated by Prior and Lyman to provide feedback about the hand aperture [114]. A single electrode provided feedback via pulse rate modulation, which resulted in an increased ability to distinguish object sizes. Another way of providing feedback about the hand aperture has been published more recently $[19,83]$. The hand aperture of a virtual hand was fed back to the subjects by moving the real index finger via a motor, which in this way provided proprioceptive motion feedback.

Despite these developments and the distinct need for hand aperture feedback, no myoelectric forearm prostheses are available today that provide any sensory feedback about the opening of the hand to the user. One of the possible reasons is the lack of proper investigation of the optimal parameters to provide the hand aperture feedback. Intuitively, the direct stimulation of the individual afferent nerves will provide the best solution to close the loop of the control of a myoelectric prosthesis, but is also the most difficult method to successfully implement in a prosthesis, due to the selectivity of the sensory system. On the other hand, vibrotactile and electrotactile stimulation seem to be of high potential to provide feedback in myoelectric prostheses, because they are easy to apply, nondisturbing to the environment and noninvasive, as already stated by Kaczmarek et al. [78]. However, no studies have been conducted on the objective comparison of these stimulation methods to provide hand aperture feedback.

Providing position feedback through an array of stimulators, in which each stimulator corresponds to a position, might be a method with higher ecological validity than methods using amplitude or frequency modulation, to provide hand aperture feedback. The phantom sensation approach of Mann and Riemers [93] can be seen as an early 
application of such an approach. Another application of an array of stimulators is presented by Antfolk et al., who used five servomotors, placed on the arm, to provide information about the different fingers to be touched [8].

In this study, the use of an array of eight stimulators to provide hand aperture feedback is investigated, whereby the methods of electrotactile and vibrotactile stimulations are compared objectively on healthy subjects and validated on a small group of patients.

\section{Methods}

\section{Subjects}

Measurements were performed on 15 healthy subjects (age, $24.6 \pm 2.9$ yrs.) and three patients (age, $45.5 \pm 9.2$ yrs., one forearm amputee, two congenital). The mean stump length of the patients was $13 \pm 5.6 \mathrm{~cm}$. Everyone was informed about the research before the start of the experiments via an information letter and all signed informed consent. Subjects were included when they had no experience with vibrotactile and electrotactile stimulation and did not have any sensory or skin problems of the arm. The study protocol has been approved by the local medical ethical committee (Medisch Ethische ToetsingsCommissie Twente).

\section{Experimental setup}

A virtual representation of an opening and closing hand (see Figure 1a) was built in Labview (Labview Inc., 2009b, National Instruments, Austin, TX) to block the normal proprioceptive pathways, thereby enabling the participation of healthy subjects. The hand aperture was controlled by the scroll wheel of an adjusted computer mouse. The "rotation clicks" were removed from the scroll wheel to avoid mechanical cues relating the mouse scroll to the hand aperture. Furthermore, a randomly varying gain between the level of mouse scrolling and the hand aperture was used to further avoid cues about the hand aperture related to the mouse scrolling.

(a)
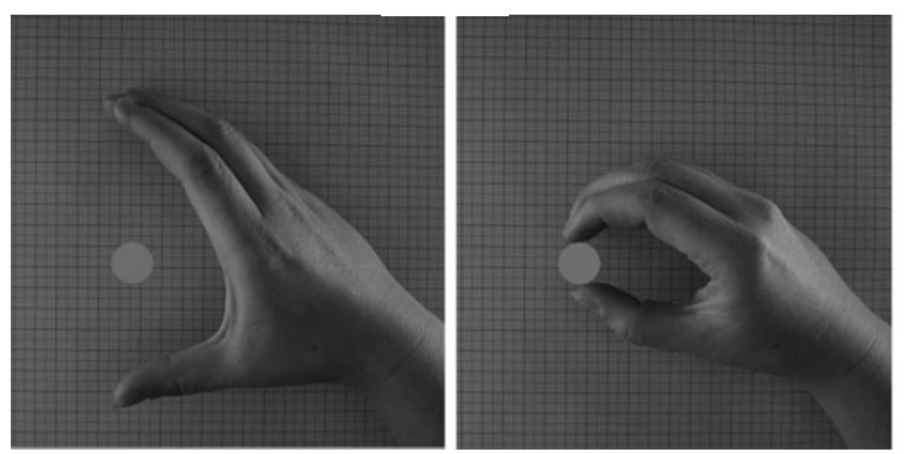

(b)

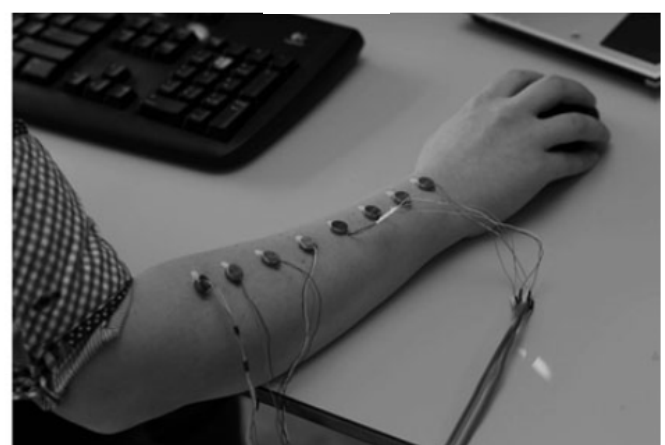

Figure 1: Experimental setup. (a) Virtual representation of the moving hand and one of the objects to be grasped. (b) Vibrotactile feedback array placed in the longitudinal orientation

Eight circular objects with varying object sizes were simulated within the Labview environment and displayed in random order (see Figure 1a). A grasping task consisted 
of the display of an object and an open hand, after which the subject had to change the hand aperture through scrolling, to a position which fitted the object correctly. When the hand aperture was held constant for $2 \mathrm{~s}$, the task was completed and another object appeared. In the visual feedback conditions, the hand and the object were visible during the whole task. For the nonvisual feedback conditions, only the object was shown shortly at the start of a new grasping task to inform the subject about the object to grasp, but not providing any visual information about the opening of the hand. For each experimental condition, 45 objects were presented, of which the first five objects were applied to get acquainted to the new condition and were not used for further analysis. All hand positions over the whole grasping motion were stored by the program.

\section{Vibrotactile and electrotactile stimulation}

Vibrotactile feedback was provided by an array of eight small commercially available coin motors (Ineed, China). These motors have been used in earlier studies on vibrotactile feedback [116] and were chosen because of their ease of use, their small size, and low costs. The coin motor consists of a rotating inner mass, which stimulates the skin in tangential direction. The frequency and force of stimulation are coupled and dependent on the characteristics of the skin to which it is attached. The driving current was primarily set to $44 \mathrm{~mA}$ for every single vibrator in each experiment, which resulted in clearly tangible, but comfortable sensations. Stimulation amplitudes were adjusted manually, if necessary, to create equally perceived amplitudes of stimulation for each stimulator. The control unit for the array of stimulators was custom build and connected to a National Instruments DAQ system (NI USB-6211, National Instruments, Austin, TX), which was controlled by a Labview syntax, incorporated in the Labview setup. The stimulators were attached to the skin by double-sided adhesive rings (EEG Kleberinge, T06, MedCat, Erica, The Netherlands).

Electrotactile feedback was provided by an array of eight small surface electrodes (Blue sensor BRS, Ambu, Ballerup, Denmark) controlled by another custom build control unit (Octostim) and via Bluetooth the stimulation commands were sent from the Labview setup to the control unit. A counter electrode (anode) was placed at the wrist. Before starting the experiments with electrotactile feedback, sensation and comfort thresholds were determined quickly for each electrode. The stimulation amplitude was increased in steps of approximately $0.035 \mathrm{~mA}$ and time intervals of $0.5 \mathrm{~s}$ until the subject felt the stimulation and pressed the stop button. The resulting amplitude was stored as the sensation threshold. After $1.5 \mathrm{~s}$ rest, the amplitude was increased again by the same steps and the subject was asked to press the stop button when the stimulation was not comfortable anymore. The resulting amplitude was called the comfort threshold. The stimulation amplitude for the experiment was determined at $60 \%$ between the sensation and comfort threshold. A 60\% threshold was chosen to get clear, but certainly not painful sensations and was based on experience in earlier experiments. The stimulation amplitude was checked for every electrode and adjusted if necessary to get an equal sensation for all electrodes. 


\section{Feedback}

Feedback was applied on the same arm as used for providing the control input to the simulated hand, which was their dominant arm in computer use. The hand aperture was fed back to the subjects by activation of the corresponding stimulator. The hand aperture was discretized to eight steps, corresponding to the eight stimulators of the array placed on the arm of the subjects. When a hand position was reached, only one of the stimulators was activated and vibrated until further movement of the hand. The stimulators were placed either in a longitudinal or transversal configuration (see Figure 2). For the longitudinal configuration, the stimulators were placed between the elbow joint and the wrist at the dorsal side of the forearm. Activation of the stimulator closest to the wrist corresponded to a fully closed hand. In the transversal configuration, stimulators were placed around the forearm. To create the largest distance between stimulators, the array was placed at the largest circumference of the forearm, but at least $3 \mathrm{~cm}$ from the elbow joint. The distance between the stimulators was equally spread and marked on the arm to have the same positions for both types of stimulation. For the patients, the stimulators in the longitudinal configuration were placed on the dorsal side of the stump, with equal inter-stimulator distances as for the transversal orientation, which led to a number of stimulators $(3,3$, and 6 for the patients) crossing the elbow and placement on the upper arm.

Besides the continuous hand aperture feedback, in some experimental conditions also feedback was provided when the hand aperture corresponded exactly to the size of the shown object, which is referred to as touch feedback. This feedback was given by an extra stimulator or electrode. This stimulator was placed on the forearm of the subject between the elbow joint and the wrist. It was placed on the dorsal side when the array was placed transversally and on the ventral side of the forearm when the array was placed in the longitudinal orientation. The amplitude of stimulation of the single vibrotactile stimulator was the same as for the stimulators in the array and the amplitude of the single electrotactile stimulator was determined by the same procedure as used for the array electrodes as described before. The extra stimulator was activated simultaneously with one of the stimulators in the array, during the whole period the hand was at the correct position.

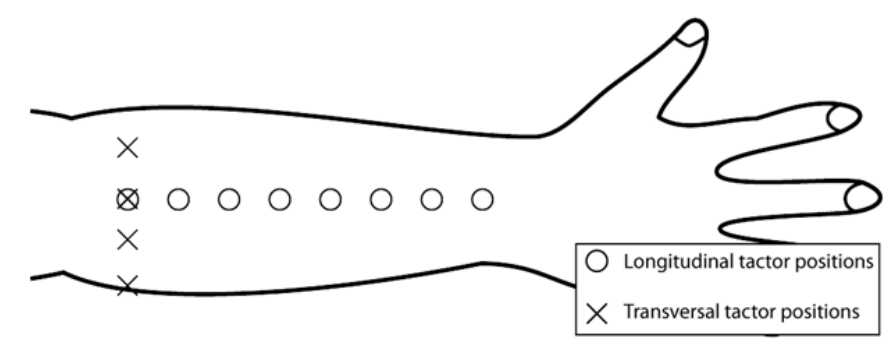

Figure 2: Schematic overview of the array orientations on the forearm 


\section{Experimental conditions}

The grasping tasks, consisting of the grasping of 45 objects, were performed under four different feedback conditions for both types of stimulation and both array orientations (see Table 1). The order of the type of stimulation applied and the order of array orientation were randomly chosen beforehand. Both array orientations were applied subsequently for one type of stimulation to reduce the time needed to switch between stimulation types.

Table 1: Summary of the four different feedback conditions used in the experiment

\begin{tabular}{|l|l|l|}
\hline $\mathbf{1}$ & Condition name & Content \\
\hline $\mathbf{2}$ & $\begin{array}{l}\text { Hand aperture } \\
\text { feedback }\end{array}$ & $\begin{array}{l}\text { The virtual object and hand are visible during the whole grasping task } \\
\text { and no other feedback is given }\end{array}$ \\
\hline $\mathbf{3}$ & $\begin{array}{l}\text { Hand aperture \& } \\
\text { touch feedback }\end{array}$ & $\begin{array}{l}\text { Comparable to hand aperture feedback, but an extra stimulator is } \\
\text { activated when the virtual hand exactly grasps the object }\end{array}$ \\
\hline $\mathbf{4}$ & No feedback & $\begin{array}{l}\text { No feedback about the hand aperture is provided and the virtual object to } \\
\text { be grasped is only shown shortly }(0.5 \mathrm{~s}) \text { at the start of the task }\end{array}$ \\
\hline
\end{tabular}

\section{Validation on patients}

Based on the results from the healthy subjects, a smaller part of the protocol was executed as validation on patients. Stimulation parameters showing the largest difference in performance were selected, but not when the performance was worse than the non-feedback situation. For every parameter setting, the visual and the hand aperture feedback situation were evaluated and the non-feedback situation was performed once at the end of the experiment.

\section{Outcome parameters and statistical analysis}

For every grasping task performed with a certain experimental condition, the time needed to perform the task was recorded. This value comprises the time taken to reach the 40 objects, while the $2 \mathrm{~s}$ of object holding were left out. Furthermore, the hand aperture in the constant holding phase and the presented object size were compared. Based on this, the percentage of correct hand apertures and the mean absolute deviation from the correct hand aperture were calculated. Finally, also the percentage of hand apertures that only deviated up to one position from the correct hand aperture was calculated. This last parameter was chosen to provide an additional accuracy measure which is less strict. A repeated measures analysis of variance (ANOVA) analysis was performed in SPSS (PASW Statistics 18, IBM, Armonk, NY) to evaluate the differences in outcome parameters. The type of stimulation (vibrotactile or electrotactile), the feedback condition (visual, hand aperture, hand aperture and touch, and no feedback), and the orientation of the stimulator arrays (longitudinal or transversal) were used as the within subjects variables. In case of significant differences $(\mathrm{p}<0.05)$, post-hoc Student t-tests were performed for all possible combinations within each factor. A Bonferroni correction was applied when multiple tests were conducted. 


\section{Results}

The results on healthy subjects will be presented first. At the end of this section, the validation results in three patients will be shown.

(a)

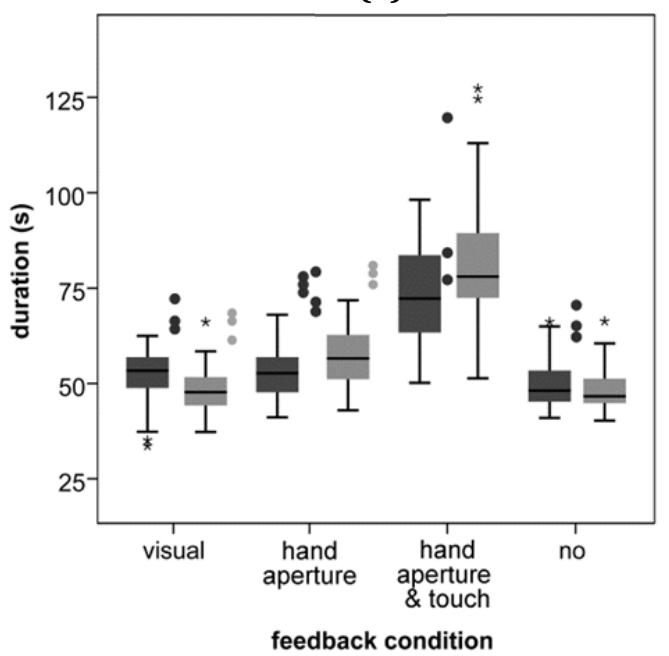

(c)

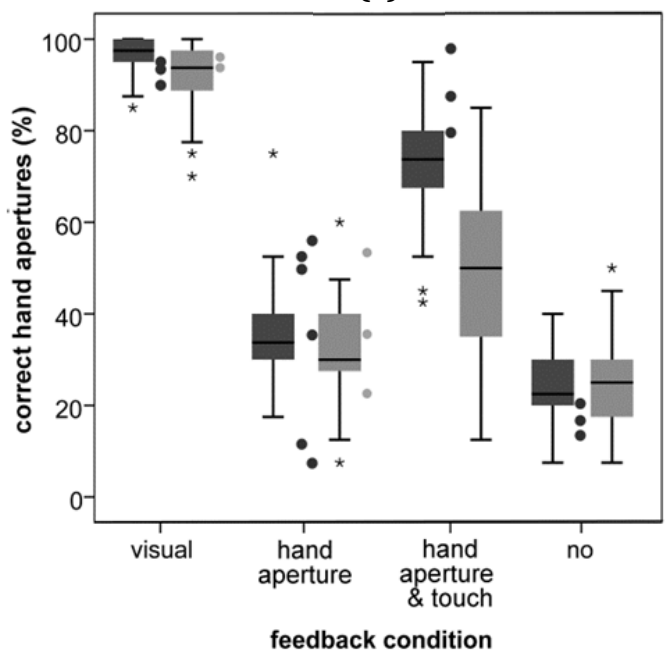

(b)

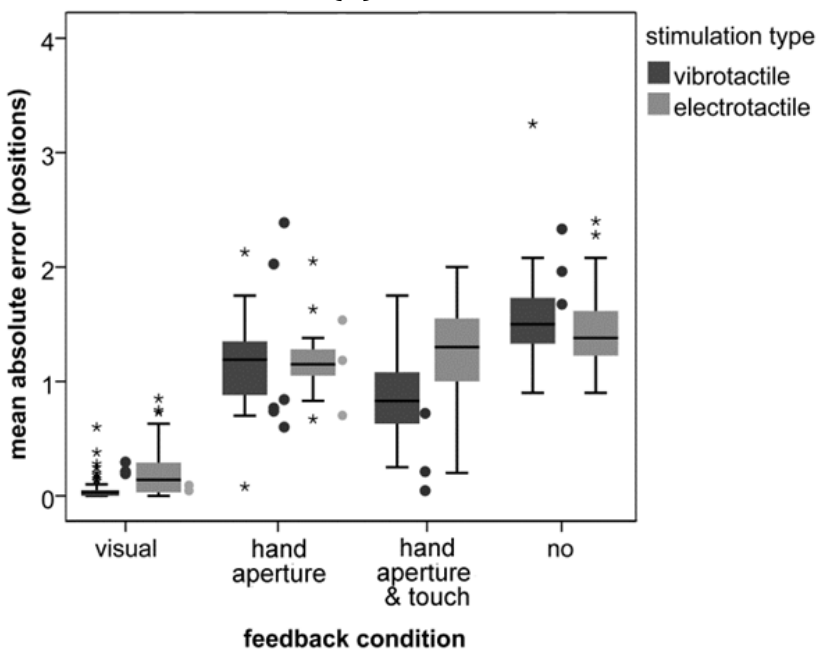

(d)

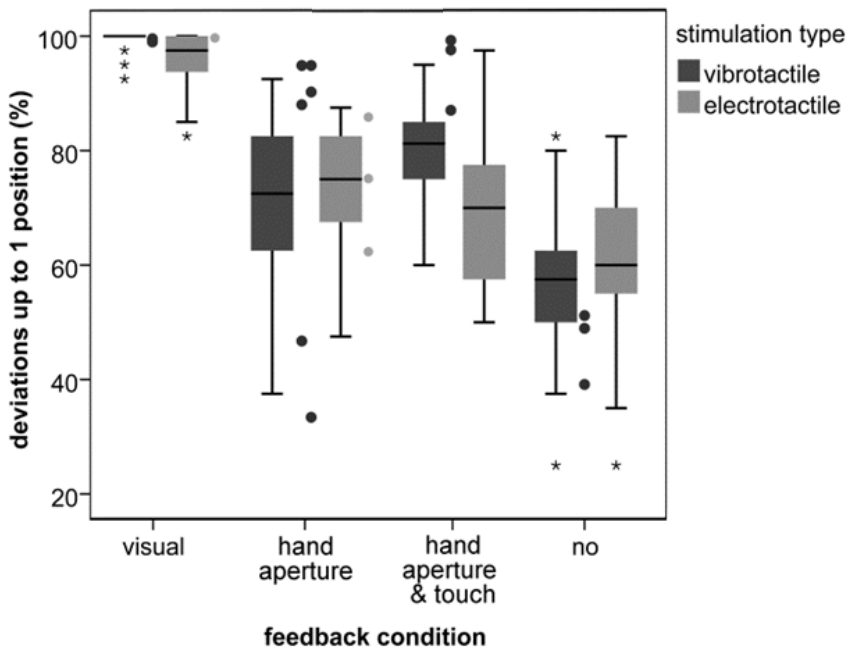

Figure 3: Distribution of (a) the duration of the tasks, (b) the mean absolute error, expressed in positions, (c) the percentage correct hand apertures, and (d) the percentage deviations up to one position, categorized per feedback condition (see Table 1) and grouped per type of stimulation for healthy subjects. Patient results are shown in dots next to the bars per feedback condition and for all four parameters. For feedback through vibrotactile stimulation, patient results are shown for both the transversal (left column) and longitudinal (right column) oriented array

\section{Descriptive statistics}

The duration of a single task, the mean absolute error, the percentage correct hand apertures, and the percentage hand apertures deviating up to one position are presented in boxplots (see Figure 3a-d). Median values of the data of all 15 healthy subjects are represented by the thick horizontal lines, the borders of the boxes are the 25th and 75th percentiles and the whiskers represent the minimum and maximum values. 
Asterisks indicate the outliers with values larger than 1.5 times the interquartile range from the box edge. Data are separated for both types of stimulation (vibrotactile and electrotactile), but combined for the orientation of the stimulator arrays.

A large spread in data is seen over the different subjects. Further statistical analysis is, therefore, performed by a repeated measures procedure. Repeated measures ANOVA was performed over all data for all four outcome parameters, with the orientation of the stimulator arrays, type of stimulation, and feedback condition as the different factors involved in the analysis. Next, the results of this ANOVA analysis and the necessary posthoc tests are described for all factors separately.

\section{Orientation of the stimulator arrays}

The distance between the stimulators was almost comparable for both array orientations $(3.9 \mathrm{~cm}$ for the longitudinal orientation compared to $3.8 \mathrm{~cm}$ in the transversal configuration). A paired t-test has shown that these differences were not statistically different $(\mathrm{p}=0.2)$. Resulting from the repeated measures ANOVA, no significant differences for all four parameters were found for the orientation of the array on the forearm ( $p=0.37$ to 0.92$)$.

\section{Type of stimulation}

No subjective measures, like questionnaires, to compare vibrotactile and electrotactile stimulations were used. However, several subjects spontaneously reported that vibrotactile stimulation was perceived as more comfortable. Furthermore, electrotactile stimuli were often reported as painful (after threshold determination) and had to be adjusted, which never happened for vibrotactile stimuli.

The repeated measures ANOVA showed significant differences for all four outcome parameters for the type of stimulation. The performance in the grasping tasks is significantly better when feedback is given via vibrotactile stimulation, expressed in a shorter duration of the task, lower mean errors, and higher percentages of correct hand apertures compared to electrotactile stimulation. A significant interaction component with the feedback condition was also shown, and therefore, the effects of the type of stimulation on the performance parameters were analyzed by repeated measures ANOVA for each feedback condition separately. The results of this comparison between vibrotactile and electrotactile stimulations were expressed in the $p$-values for significance and summarized in Table 2.

Table 2: p-values of repeated measures ANOVA for each outcome parameter with type of stimulation as factor, per feedback condition separately

\begin{tabular}{|l|l|l|l|l|}
\hline Feedback & Duration & Percentage correct & Mean error & Percentage error $\leq 1$ \\
\hline Hand aperture & $\mathrm{p}=0.024^{*}$ & $\mathrm{p}=0.283$ & $\mathrm{p}=0.771$ & $\mathrm{p}=0.291$ \\
\hline Hand aperture \& touch & $\mathrm{p}=0.16$ & $\mathrm{p}<0.001^{*}$ & $\mathrm{p}=0.001^{*}$ & $\mathrm{p}=0.002^{*}$ \\
\hline No & $\mathrm{p}=0.523$ & $\mathrm{p}=0.343$ & $\mathrm{p}=0.397$ & $\mathrm{p}=0.389$ \\
\hline
\end{tabular}

When only hand aperture feedback is provided, there is no difference in performance between feedback through vibrotactile or electrotactile stimulation, while vibrotactile 
stimulation performs much better when extra feedback is added about the exact grasping. Although there were no differences in the direct performance parameters, the duration of the tasks performed with vibrotactile stimulation was significantly lower compared to electrotactile stimulation in the situation with only hand aperture feedback.

\section{Feedback conditions}

The results of the repeated measures ANOVA show significant differences in performance over the four feedback conditions (visual, hand aperture, hand aperture and touch, and no feedback) for all four performance parameters $(p \leq 0.001)$. A significant interaction component with the type of stimulation was also seen; therefore, repeated measures ANOVA was performed per type of stimulation, which showed significant influences of feedback condition for both types of stimulation for all four outcome parameters. Therefore, the post-hoc analyses were performed for both types of stimulation separately. To compensate for the repeated execution of post-hoc tests, Bonferroni correction was applied. The original significance level $(0.05)$ is divided by the number of tests performed (6). The corrected significance is now 0.008 .

No differences were found for the duration of the tasks in conditions without any feedback compared to tasks where visual feedback is available. However, the addition of feedback through electrotactile stimulation increases the duration of the tasks significantly $(\mathrm{p}<0.001)$ compared to the non-feedback and visual feedback conditions, which is also the case for the addition of touch feedback $(\mathrm{p}<0.001$ for electrotactile stimulation as well as for vibrotactile stimulation). It took the subjects longer to identify the activation of the extra stimulator or they needed more time to reach the correct hand aperture.

The percentages hand aperture with deviations up to one position show a significant increase in performance when feedback is added. The best performance is shown for the visual feedback condition, followed by the hand aperture and touch feedback condition. The performance in conditions with hand aperture feedback is significantly better compared to the non-feedback conditions $(\mathrm{p}=0.001$ and $\mathrm{p}=0.003$ for electrotactile and vibrotactile stimulations, respectively). For the electrotactile stimulation feedback conditions, no increase in performance is seen for the addition of touch feedback compared to the hand aperture feedback condition ( $p=0.135$ compared to $p=0.007$

for vibrotactile hand aperture and touch feedback). The same conclusions can be drawn for the other outcome parameters, the mean absolute error, and the percentage of correct hand apertures, which showed comparable $p$-values.

\section{Validation on patients}

The stimulation conditions used in the validation protocol were (1) the transversal oriented vibrotactile array, (2) the longitudinal oriented array, (3) vibrotactile touch feedback with the transversal oriented array, and (4) the transversal oriented electrotactile array. The results are plotted in Figure 3 for each patient separately and each feedback condition. On average, the performance parameters were highly 
comparable with healthy subjects and for the transversal vibrotactile array even somewhat higher (up to 16\%). Furthermore, the same trends were seen, specifically an increase in performance with feedback and a higher duration for electrotactile compared to vibrotactile feedback and for touch feedback. However, the duration of all tasks was higher for the patients (70.6 compared to $55.4 \mathrm{~s}$ ).

\section{Discussion}

\section{Orientation of the stimulator arrays}

The distance between the stimulators was not significantly different between both orientations of stimulation. Therefore, performance parameters could be compared between these two orientations. The longitudinal orientation was selected for this study because it is possibly more functional and has a more intuitive relation to the control of the hand and the orientation of the muscles used to close and open the hand. However, the forearm stump could be too short to apply an array of eight stimulators in a longitudinal direction, which is not the case for the transversal oriented array.

Our results showed no differences in performance for the different orientations of the stimulator array. However, differences in distance and location perception were found by Green between both orientations of pressure stimuli [62], and in a study by Higashiyama and Hayashi better localization performance of seven electrotactile stimuli was seen for transversal oriented arrays [70]. In addition, Cody et al. showed that the spatial acuity is significantly better for stimulators oriented transversal on the arm compared to longitudinal [41]. These findings were explained by the orientation of the receptive fields of the mechanoreceptors in the forearm. These fields are smaller in the transversal direction compared to the longitudinal direction. However, no effects of this difference were found in our study. It is also known that the localization of vibrotactile stimuli, which is also used in these experiments, is better for stimulators close to an anatomical landmark [35]. Some of the longitudinal oriented stimulators were at the ends close to these landmarks (wrist and elbow), but for the transversal orientations these effects are equal over the whole length. This could have counteracted possible differences in performance caused by the asymmetry in receptive fields. Furthermore, the experiments of Cody et al. were performed with a von Frey hair stimulus at shorter inter-stimulus intervals, which also made comparison with our study difficult.

The mean distance between the stimulators was less than $4 \mathrm{~cm}$, which is within the spatial acuity range found in the literature for different body locations $(2-4 \mathrm{~cm})$ [31]. However, most psychophysical studies were performed using pressure stimuli instead of vibrotactile stimuli and not with this number of stimulators. Higashiyama and Hayashi used an array with seven electrodes on the volar side of the forearm and found localization errors that were much smaller than the inter-electrode distance, used in our study [70]. In a study by Cholewiak et al. [34], it was shown that the maximum number of stimulators to be distinguished on the trunk was seven. The optimal number of stimulators to be used in an array on the forearm should be further investigated, where a tradeoff should be made between the localization performance and the amount of 
information (number of hand aperture levels for example) to be fed back by the stimulation.

\section{Type of stimulation}

A significant effect was found for the type of stimulation. In general, all performance outcome parameters were better for the experimental conditions with feedback through vibrotactile stimulation compared to electrotactile stimulation. The only exceptions were the hand aperture feedback conditions without touch feedback. In these cases, the percentages of correct hand apertures, the mean error, and the percentages deviations up to one position were comparable for both types of stimulation, indicating the same level of accuracy that could be achieved. However, the time needed to perform the grasping tasks was significantly higher for the electrotactile stimulation condition, which also impedes the performance, because this will slow down the handling speed of the subjects. This difference in duration can be caused by lower onset times of the vibrotactile stimulators or because it takes longer to recognize a specific electrotactile stimulator in an array. However, these hypotheses have not been investigated further or been described earlier. In recent research, there is already a clear preference for the use of vibrotactile stimulation over electrotactile stimulation. Vibrotactile stimulation has been indicated as a more comfortable stimulation method [116] and the small range between sensation and pain thresholds with electrotactile stimulation was also indicated as a reason to prefer vibrotactile stimulation over electrotactile stimulation [76]. However, it has not been objectively investigated before whether the performance with vibrotactile stimulation is better than with feedback through electrotactile stimulation.

The addition of an extra stimulator for touch feedback further increases the performance in the grasping tasks when vibrotactile feedback is provided, but the performances stay on a constant level for electrotactile stimulation. This was also indicated by the subjects, who stated that it was difficult to experience the difference between the activation of one of the electrodes within the array and activation of the extra electrode. However, the distance between the stimulator array and the extra stimulator was always larger than the inter-stimulator distance of the array. No clear differences in spatial acuity and localization can be found in the literature for electrotactile and vibrotactile stimulation, but this study showed that differentiation problems can occur when a single electrotactile stimulator is placed too close to an array of stimulators and activated at the same moment. Increasing the stimulation amplitude might enhance the differentiation between the array and the extra stimulator, but also increases the likelihood of painful stimuli.

The currently available vibrotactile stimulators are mostly not suitable for applications in forearm prostheses, because of their relatively large size and high power consumption. The coin motors, as used in this study and in a study by Pylatiuk et al. [116], can be a good alternative, because of the small size and low costs. A comparison of localization performance with a dense array of these coin motors and larger C2 tactors showed no differences between these stimulators [33]. 
However, the actual performance and application in forearm prostheses should be an important subject for further research.

\section{Feedback conditions}

The best results for the percentages of correct hand apertures and the mean absolute errors were shown for the hand aperture and touch feedback conditions. However, the duration of the tasks was also significantly increased with the addition of feedback through electrotactile stimulation and the use of the extra stimulator, which is in line with the recent literature showing that the addition of proprioceptive feedback increases the performance, but at the cost of a longer task execution duration $[15,83$, $140,155]$. Vibrotactile feedback enhances the performance in grasping tasks without lengthening of the task duration and can be seen as a better method to provide hand aperture feedback compared to feedback through electrotactile stimulation or the use of an extra stimulator.

The percentages of correct hand apertures that can be achieved by the addition of vibrotactile or electrotactile feedback are quite low, between $30 \%$ and $50 \%$, especially when compared to the visual condition where percentages of almost $100 \%$ can be reached. However, the increase in performance that can be achieved compared to the non-feedback condition is significant. Furthermore, it was shown that the desired hand aperture was reached exactly or with a deviation of one position in almost $80 \%$ of the cases. This shows that the addition of hand aperture feedback significantly improves the accuracy in a grasping task. In a study by Blank et al., it was already shown that feedback about the hand aperture improves the targeting accuracy compared to non-feedback conditions [20]. However, they have used proprioceptive motion feedback, moving the index finger according to the movements of a virtual finger and controlled by the force applied by the thumb, which cannot be used by patients. The aim of their study was to investigate the general effects of proprioceptive feedback. They concluded that proprioceptive feedback indeed increases the accuracy in coarse movements, but the addition of tactile feedback would be needed to improve the accuracy in more precise movements. This was confirmed by the results of our study in which the percentage of correct hand apertures could still be significantly improved by the addition of an extra stimulator, activated when the virtual object was touched. This touch feedback may be combined with feedback about the grasping force and thereby further increase the performance in grasping tasks.

\section{Validation on patients}

The performances of patients in virtual grasping tasks were comparable to the results with healthy subjects, despite the possible differences in sensibility of the stums and the fact that control of the hand was performed with the other hand. The placement of a number of stimulators on the upper arm for the longitudinal orientation did not influence the results and can be a solution for patients with short stumps. In actual use, the prosthesis will be controlled by EMG. In the current study, EMG control was replaced by scroll wheel control in order to focus on the influence of tactile feedback on 
performance, avoiding the confounding effect of EMG control, which may be very variable amongst. However, in future studies, EMG control needs to be included. An increase of duration of the tasks was seen in comparison to the healthy subjects, which could be caused by the difference in experience with experimental settings and computer skills. The healthy subjects all did participate in experimental studies before and all patients did not. However, it is also possible that patients needed more time to interpret the stimuli.

\section{Experimental setup}

Because of the relatively small number of patients available, the largest part of the experiments is performed on healthy subjects. Therefore, a virtual environment, showing an opening and closing hand, was built to circumvent the intact proprioceptive pathways of the healthy subjects. The hand was controlled by the scroll wheel of a computer mouse, which is not comparable to the myoelectric control as used in today's prostheses. This approach was chosen to avoid the long training period needed to learn the myoelectric control and to avoid the large variability in the within-subject performances for this control method. The muscles used to control the computer mouse are to some extent comparable to those used to open and close the hand. Furthermore, it also ensures that no feedback about the hand aperture could be derived via channels other than the stimulation provided. To further optimize this, the click mechanism was removed from the scroll wheel of the computer mouse and a variable gain between the scrolling movement and the hand movement was added. The success of this approach was shown in the results for the non-feedback conditions. The percentages of correct hand apertures were around $20 \%$, which coincides largely with the percentages expected with straight guessing. The percentages are somewhat higher, due to the known endpoints of the hand movement when the hand is fully closed or opened.

\section{Conclusion}

It is shown that feedback about the hand aperture through vibrotactile and electrotactile stimulation improves the performance in grasping tasks for healthy subjects as well as for potential users, being forearm amputees using myoelectrically controlled prostheses. This performance is expressed in an increase of correct or nearly correct hand apertures and a decrease in absolute errors. Future applications of vibrotactile stimulation are preferred over electrotactile stimulation, because the duration of the tasks is undesirably increased with electrotactile stimulation. The addition of touch feedback leads to even more accuracy for vibrotactile feedback, but also increases the duration of the grasping tasks. 


\section{Chapter 3 - Vibrotactile hand aperture feedback and distraction}

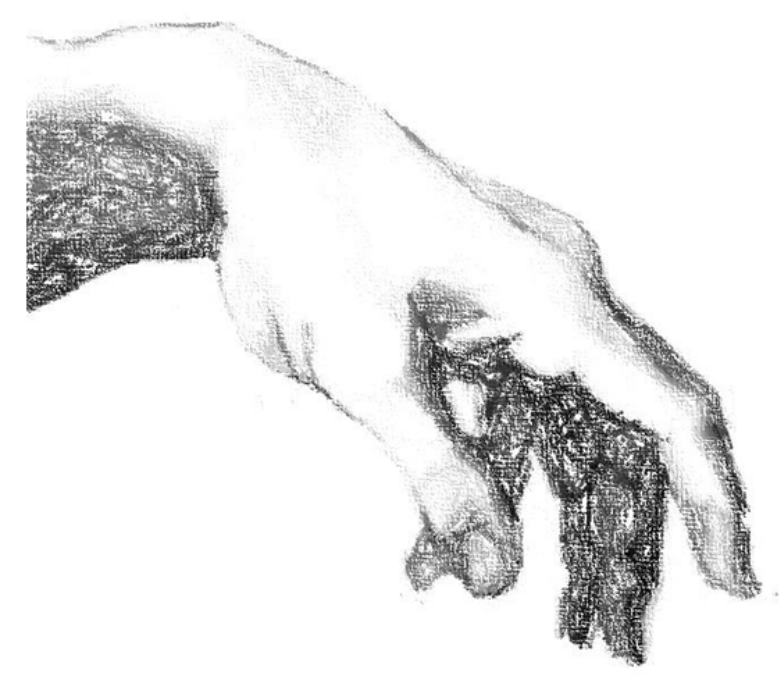

Published as: Hand-opening feedback for myoelectric forearm prostheses:

Performance in virtual grasping tasks influenced by different levels of distraction

Authors: Heidi Witteveen, Leonie de Rond, Hans Rietman, Peter Veltink

In: Journal of Rehabilitation Research and Development, vol.49. no. 10, pp. 15171526, 2012 


\begin{abstract}
Sensory feedback and the required attentional demands are important aspects in prosthesis acceptance. In this study, hand aperture feedback is provided and the performance in a virtual grasping task is investigated. Simultaneously, a secondary task was performed to investigate the attentional demands. Ten nondisabled subjects performed the tasks with and without feedback about the hand aperture through an array of eight vibrotactile stimulators on the forearm. Activation of one stimulator corresponded to one level of hand aperture. For the dual-task experiments, subjects simultaneously performed a secondary auditory counting task. The addition of vibrotactile feedback increased the performance (expressed in percentages of correct hand positions, mean absolute errors in position, and percentages of deviations up to one hand-opening position), but the duration of the tasks was also increased. Three levels of distraction (no distraction, counting task, count and subtract task) were applied, which did not influence the performance in the grasping tasks except for the highest level of distraction. We concluded that the proposed method to provide hand aperture feedback through an array of eight vibrotactile stimulators is successful because the performance in a grasping task increases, but is not significantly attention demanding.
\end{abstract}




\section{Introduction}

The introduction of myoelectric prostheses has led to the development of prostheses with higher levels of functionality. However, these kind of prostheses lack the ability to provide the prosthesis user with adequate sensory information [110]. In contrast with body-powered prostheses, where the control cables can provide force and some position feedback [111], the visual and, to some extent, auditory sensory systems are the only systems that provide some sensory awareness in myoelectric prostheses. This causes a high mental burden on the user to continuously use his or her visual sensory system to control the prosthesis [143]. Pons et al. showed that because of the limited controllability and burden on the visual system, 30 to 50 percent of the prostheses are not used on a regular basis [113]. Adding sensory feedback, and thereby reducing the required visual attention, has been indicated to be an important improvement in upperlimb prostheses to increase acceptability [12, 113]. Furthermore, Peerdeman et al. concluded from a workshop with representative prosthesis users that feedback should be added to reduce the attentional demands and allow intuitive grasping [107]. Feedback about the grasping force and hand aperture were shown to be especially important and should be incorporated in future upper-limb prostheses.

Only a few studies have investigated the use of position feedback in upper-limb prostheses. In an early approach by Mann and Reimers, vibrotactile stimulation was used to provide feedback about the elbow angle [93]. They placed two stimulators on the upper arm and activated them simultaneously with differing amplitudes. The ratio of the amplitudes determined the location in between the stimulators where the stimulation was perceived (concept of phantom sensations). Although their results were promising, no applications of this method can be found. Furthermore, large vibrators and a stabilization plate were necessary to create the sensations [6]. Dhillon and Horch undertook another approach to provide feedback about the elbow angle; they used implanted electrodes to stimulate the individual afferent nerves [50].

Prior and Lyman incorporated hand aperture feedback by using one single electrode to provide feedback about the grasping force via pulse width modulation and about the hand aperture via pulse rate modulation [114]. Although they showed that hand aperture feedback increases the ability to distinguish object sizes, no applications are found in current prostheses. One reason for this could be the small range between sensation and pain thresholds with electrotactile stimulation, which results in small stimulation ranges $[76,78]$.

Recent approaches have focused on proprioceptive feedback about the movement of the index finger in a grasping movement $[19,83]$. A virtual index finger was controlled by force input from the thumb while feedback about the position of the index finger was provided by moving the real index finger with a motor. Although not directly applicable in forearm prostheses, these studies show that proprioceptive feedback increases the awareness of finger movements.

Not only the lack of feedback can lead to the abandonment of the myoelectric prosthesis, but also the required attention and concentration needed to control the prosthesis [11]. As stated by Cipriani et al., "Acceptability is more dependent on the required attention 
than on the success in grasping" [40]. The addition of feedback creates an extra level of attention required to control the prosthesis, which should be kept as low as possible. In the ideal situation, the feedback can be used subconsciously without requiring extra attention, and extra external distraction would not significantly influence the performance with the prosthesis. None of the previously mentioned studies on position feedback have investigated the attentional demands of the feedback. A way to investigate these attentional demands is the use of dual-task methodology [72,160]. It is assumed that there is a fixed processing capacity for each individual [125], which is (partly) filled when feedback is used to control the hand aperture of a prosthesis. The capacity used determines the attentional demand of the task. The addition of a secondary task requires extra processing capacity to perform both tasks. A high attention-demanding primary task will require a large part of the processing capacity, thereby reducing the available capacity for the secondary task. Therefore, the performance of the secondary task is an indicator of the attentional demand of the primary task. A restriction for these experiments is that the performance of the primary task should be kept constant, which is not always possible. To solve this, researchers evaluated the performance of the primary task while keeping the performance of the secondary task constant. Stepp and Matsuoka showed an example of this approach [140]. They evaluated the effect of distraction on an object displacement task in which feedback was provided about the force that was applied by the subject. The secondary task they used was an auditory calculation task. Their results showed a decrease in speed and an increase in difficulty but no effect of distraction on their main outcome parameter for the performance of the primary task.

In this study, we placed an array of eight vibrotactile stimulators on the forearm to provide feedback about the hand aperture of a prosthesis. Our aim was to evaluate the improvements in performance in a grasping task that could be achieved by this feedback method and its attentional demands. We investigated the latter using dual-task methodology.

\section{Methods}

\section{Subjects}

All experiments were performed with 10 nondisabled subjects ( 6 male and 4 female; age $25.2 \pm 2.7$ yr. [mean \pm standard deviation]). All subjects already had some experience with the experimental setup and vibrotactile stimulation for at least $30 \mathrm{~min}$, because they all took part in an earlier experiment in which the same setup was used. In the previous study, we evaluated the performance with vibrotactile and electrotactile feedback applied in varying orientation settings [157]. Typically, the time between practice and the previous experiment was between 0.5 and 4 weeks, except for one subject who performed a training session of 30 min before the start of the current experiment. 


\section{Vibrotactile feedback}

Vibrotactile feedback about the hand aperture was given by an array of eight small commercially available coin motors (iNEED (HK) Limited; Shenzhen, China). We chose vibrotactile feedback because it is easy to apply, nondisturbing to the environment, and noninvasive [78]. Furthermore, we chose the coin motors because of their small size and low cost. The coin motors already showed positive results in a study on force feedback by Pylatiuk et al. [116]. We attached the vibrotactile stimulators to the forearm of the subjects using double-sided adhesive rings (EEG Kleberinge, GVB-geliMED KG; Bad Segeberg, Germany). The stimulators were controlled by a custom-made current stimulator on batteries, and their separate activation was controlled with a LabVIEW application (National Instruments; Austin, Texas) built for this purpose. The driving current of the stimulators was primarily set to $44 \mathrm{~mA}$ for each stimulator, which resulted in clearly tangible, but comfortable, sensations. We checked perceived stimulation strengths and adjusted the stimulation amplitudes if necessary to create equally perceived amplitudes of stimulation for each stimulator. The stimulators were equally distributed over the dorsal side of the forearm between the wrist and elbow (Figure 1). The resulting distance between the center of the stimulators was $3.0 \pm 0.2$ $\mathrm{cm}$. For each hand position, we activated one single stimulator. Activation of the first stimulator (near the elbow) corresponded with a completely opened hand and activation of the last stimulator (at the wrist) corresponded with a fully closed hand. Activation of the intermediate stimulators was equally distributed over the range of hand movements.

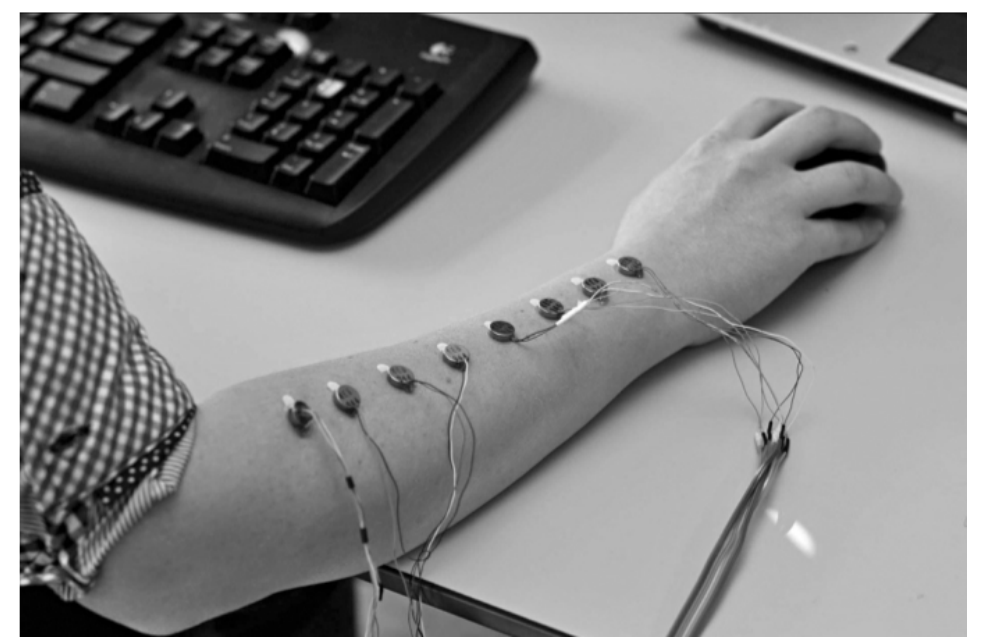

Figure 1: Experimental setup. Eight coin motors placed in an array on the forearm

\section{Virtual environment}

In LabVIEW we created a virtual representation of a closing and opening hand (Figure 2). The hand aperture was controlled by using the scroll wheel of an adjusted computer mouse. We removed the clicks normally felt during scrolling and randomized the gain between the scrolling and the resulting hand aperture to avoid information coming from the mouse movement about the hand aperture. Eight virtual objects (filled circles) with sizes corresponding to the eight hand positions, between minimal and maximal hand 
aperture, were also simulated on the screen. A series of 45 randomly selected objects comprised one task for each experimental condition. At the start of a task, an object was shown for $0.5 \mathrm{~s}$, then it disappeared. We instructed the subjects to open or close the hand to grasp the displayed object by scrolling the mouse and hold the hand in the position that they perceived as the correct position for $2 \mathrm{~s}$. After these $2 \mathrm{~s}$, another object was displayed.
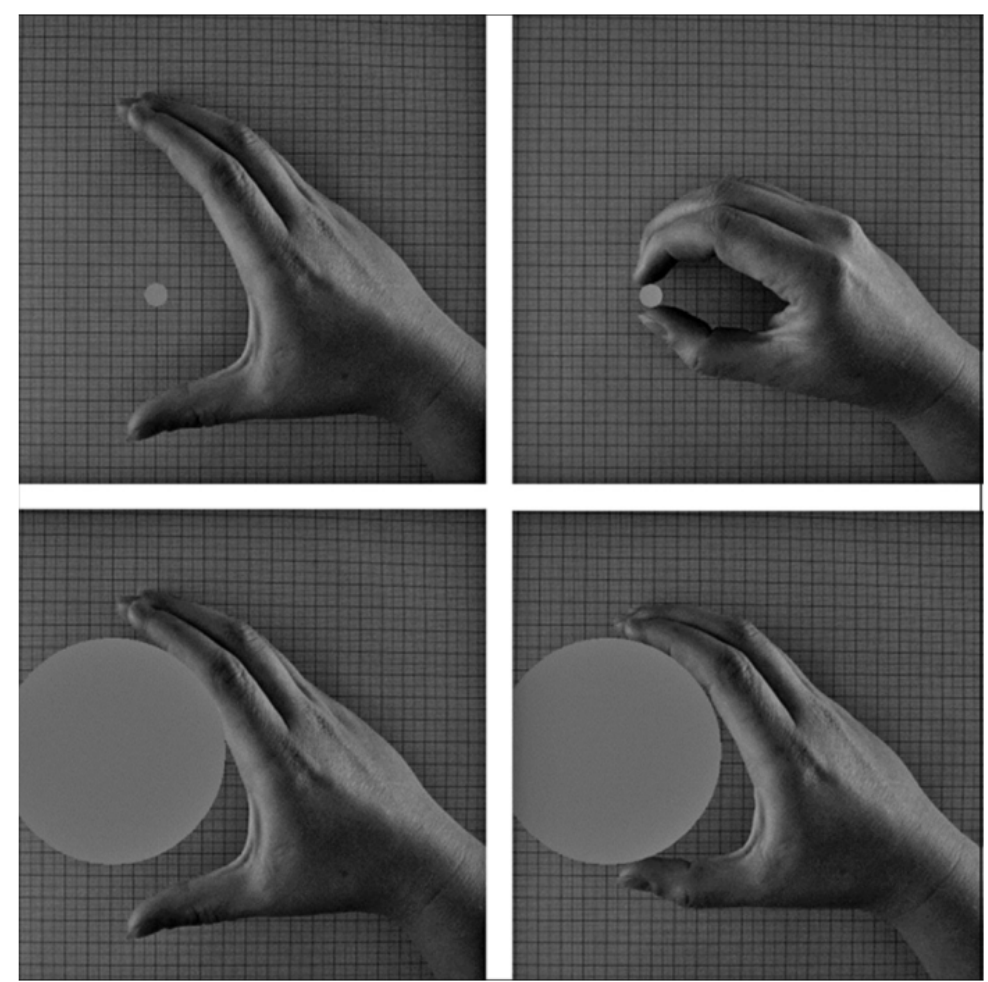

Figure 2: Virtual representation of opening and closing hand grasping the smallest and largest possible virtual objects

\section{Experimental procedure}

We sat the subjects comfortably in a quiet room, behind a laptop computer screen showing the virtual environment. Subjects controlled the computer mouse with the arm they usually used to control a computer mouse, and we placed the stimulators on the same arm. The subjects wore headphones to block the auditory cues about the position of the stimulator being activated and to present the auditory stimuli used in the secondary task.

We set up a double-task procedure to evaluate the effect of distraction on the performance in a grasping task while feedback was provided. The primary task was the grasping task, performed with and without vibrotactile feedback about the hand aperture. In both cases, the moving hand was not visible and therefore no visual feedback could be used. The secondary task was an auditory counting task. We chose an auditory task to avoid interference of the secondary task with the primary task. Beeps with low, middle, and high tones were presented randomly and within random time intervals to the subjects while they performed the grasping tasks. We created three different levels of distraction. For the lowest level of distraction (level 1), the beeps were 
presented without requiring any action by the subject. For the second level of distraction, middle and high beeps were presented and the subject had to count the number of high beeps. After completing the task, the subject reported the total number of high beeps. The third level of distraction was achieved by presenting low, middle, and high beeps and asking the subject to continuously add the number of high beeps and subtract the number of low beeps. Subjects performed every secondary task twice, with and without the help of vibrotactile feedback during the grasping task. In both conditions, visual feedback was not available, forcing the subjects to fully rely on the feedback. Subjects performed five complete training tasks with the moving hand visible on the computer screen to get used to the distraction and the feedback before the real experiment. The number of objects and the prescribed goals of these tasks were the same as those for the experimental tasks. The first five objects in every experimental condition were also used for extra training and to get used to the change in experimental conditions. We did not incorporate data from these objects in further analysis. Table 1 shows a summary of the experimental conditions.

Tasks 6 to 13 were randomized over the subjects to cancel out the effect of training on the performance in the grasping tasks. After each task, we asked the subject to indicate the perceived difficulty of the task on a visual analog scale (VAS). The VAS, ranging from "very easy" to "very difficult," was shown on the screen and the subject placed the cursor at the perceived difficulty.

\section{Data analysis}

The outcome parameters of the experiments were: (1) the duration of the task, (2) percentage of correct hand positions, (3) mean absolute position error, and (4) percentage of achieved hand positions with a deviation up to one position. These parameters are all indicators of the performance of the subject in a grasping task. We also used the number of mistakes made in the auditory task as an outcome measure to check whether the subject adequately performed the secondary task.

For all four outcome measures, we evaluated differences over the experimental conditions with a repeated measures analysis of variance (ANOVA). The factors in this analysis were the feedback condition (vibrotactile or no feedback) and the distraction level. Specific differences were post-hoc evaluated on significance by separate Student ttests. Bonferroni correction on the significance level was applied to correct for the number of repeated tests. 
Table 1: Experimental conditions

\begin{tabular}{|c|l|c|l|}
\hline Task & \multicolumn{1}{|c|}{ Feedback } & $\begin{array}{c}\text { Distraction } \\
\text { (level) }\end{array}$ & Comment \\
\hline 1 & No primary task & 2 & Training \\
\hline 2 & No primary task & 3 & Training \\
\hline 3 & Visual & - & Training \\
\hline 4 & Visual and vibrotactile & - & Training \\
\hline 5 & Vibrotactile & - & Training \\
\hline 6 & Vibrotactile & 1 & Experiment \\
\hline 7 & Vibrotactile & 2 & Experiment \\
\hline 8 & Vibrotactile & 3 & Experiment \\
\hline 9 & Vibrotactile & - & Experiment \\
\hline 10 & - & - & Experiment \\
\hline 11 & - & 1 & Experiment \\
\hline 12 & - & 3 & Experiment \\
\hline 13 & - & Experiment \\
\hline
\end{tabular}

\section{Results}

We calculated percentages of correct hand positions for each task from the desired and actual hand positions. Figure 3a shows the distribution of these values over all subjects, categorized for the different levels of distraction and grouped for both vibrotactile feedback conditions. Figures $3 \mathrm{~b}$ and $3 \mathrm{c}$ show the distribution of the percentages of hand positions with deviations up to one position and the mean absolute error. The duration of the tasks, without the $2 \mathrm{~s}$ of stable grasping per object, is shown per distraction level and feedback type in Figure 3d.

Table 2: Analysis of variance (ANOVA) for all four outcome parameters with feedback and distractions as factors

\begin{tabular}{|c|c|c|c|}
\hline ANOVA & df & $\mathbf{F}$ & p-value \\
\hline \multicolumn{4}{|c|}{ Percentage of correct hand apertures } \\
\hline Feedback & 1 & 34.09 & $0.00^{*}$ \\
\hline Distraction & 3 & 1.80 & 0.17 \\
\hline Feedback $\bullet$ Distraction & 3 & 3.06 & $0.05^{*}$ \\
\hline \multicolumn{4}{|c|}{ Percentage of deviations up to one position } \\
\hline Feedback & 1 & 75.83 & $0.00^{*}$ \\
\hline Distraction & 3 & 0.88 & 0.46 \\
\hline Feedback $\bullet$ Distraction & 3 & 1.69 & 0.19 \\
\hline \multicolumn{4}{|c|}{ Mean of absolute error in positions } \\
\hline Feedback & 1 & 36.59 & $0.00^{*}$ \\
\hline Distraction & 3 & 0.83 & 0.49 \\
\hline Feedback $\bullet$ Distraction & 3 & 2.87 & 0.06 \\
\hline \multicolumn{4}{|l|}{ Duration } \\
\hline Feedback & 1 & 14.92 & $0.01^{*}$ \\
\hline Distraction & 3 & 3.00 & $0.05^{*}$ \\
\hline Feedback•Distraction & 3 & 1.36 & 0.28 \\
\hline
\end{tabular}


(a)

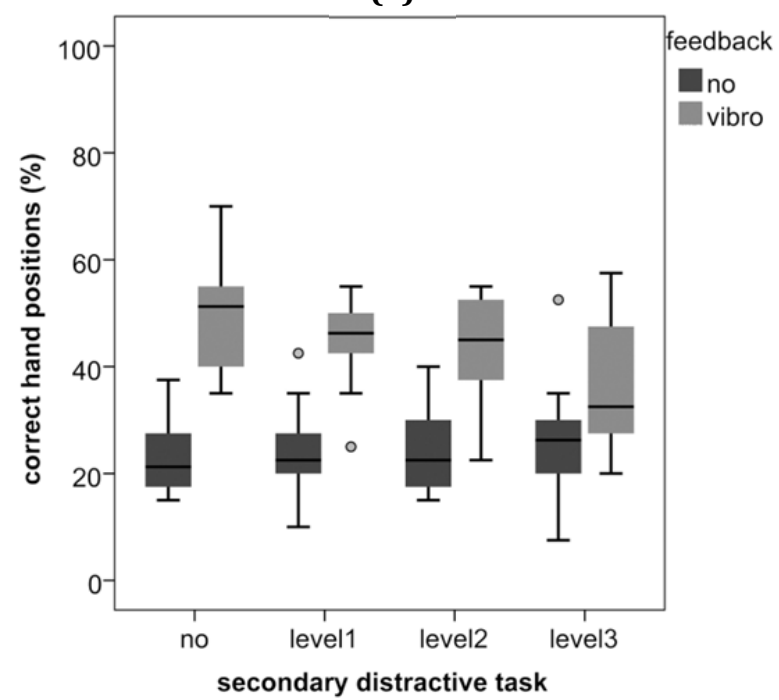

(c)

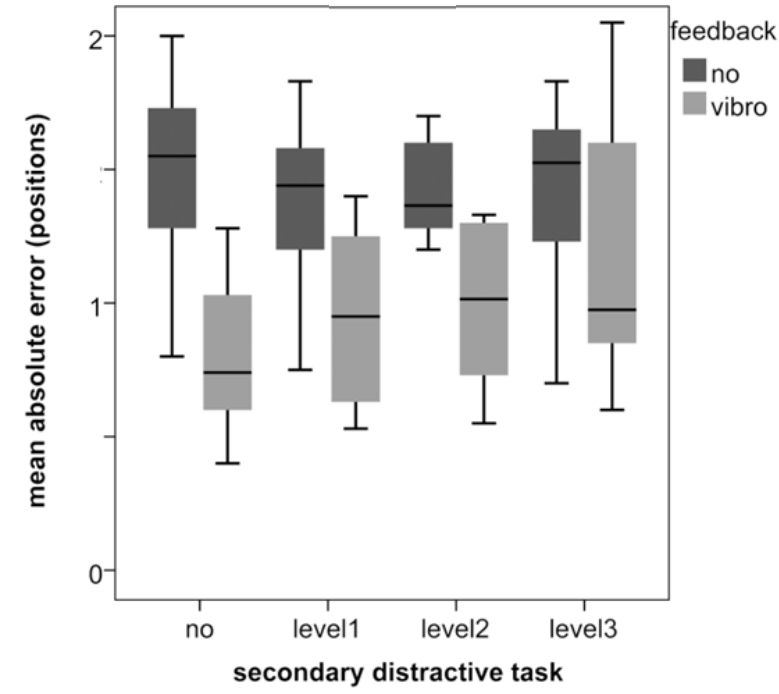

(b)

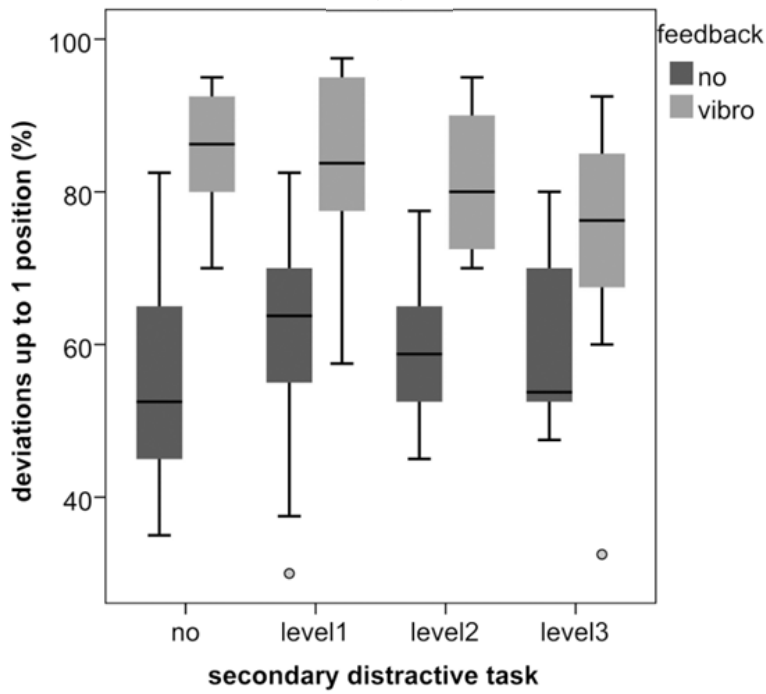

(d)

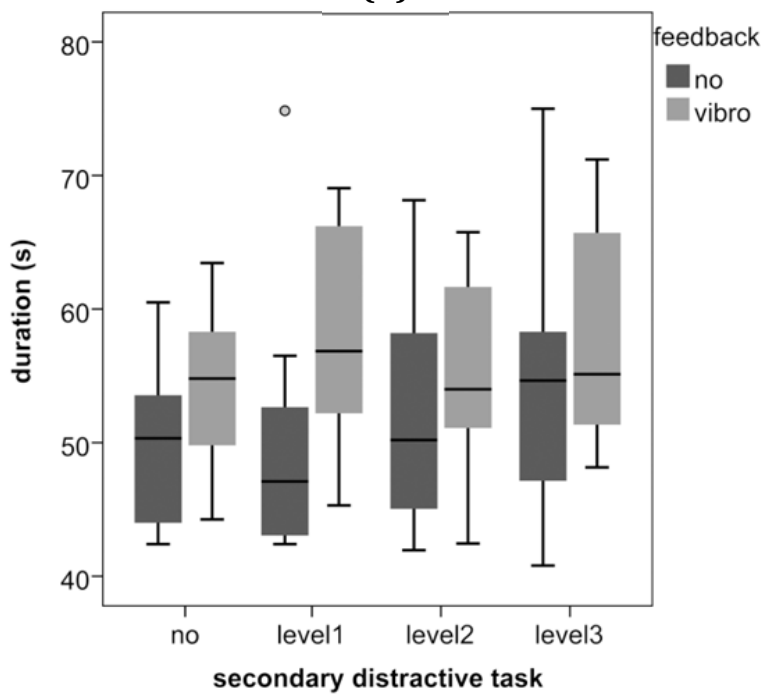

Figure 3: Distribution of (a) percentages of correct hand positions, (b) percentages of deviations up to one position, (c) mean absolute error, and (d) duration of tasks per distraction level and feedback type (Table 1). Horizontal bars represent median value, outer sides of boxes represent 25 and 75 percentiles, and whiskers represent minimum and maximum values. Small circles represent outliers with values larger than 1.5 times interquartile range from box edge. Vibro $=$ vibrotactile

We analyzed the effects of the vibrotactile feedback and the distractive secondary task on the four outcome parameters with repeated-measures ANOVA. The corresponding ANOVA tables are shown for all parameters, and significance is indicated at the 95 percent level (Table 2).

Feedback shows a significant effect on all four performance parameters. The addition of vibrotactile feedback increases the percentage of correct hand positions and the percentage of hand positions with a deviation up to one position. The mean absolute error between correct and derived hand positions is significantly decreased. However, the addition of feedback also significantly increases the time needed to complete the task. The duration of the tasks was also significantly influenced by distraction, but we 
saw no effect of distraction for the other performance parameters. However, for the percentage of correct hand positions, we found a marginally significant interaction for effect of feedback and distraction. To evaluate this interaction, we evaluated the effects of distraction for both feedback conditions separately.

Distraction was shown to have a significant effect $(p=0.01)$ on the percentage of correct hand positions only in the vibrotactile feedback conditions $(p=0.81$ for the nonfeedback conditions). We compared performances at a given level of distraction with the experimental conditions without any distraction and evaluated them by post-hoc analysis. $P$-values were $0.18,0.08$, and 0.01 , respectively, for the three levels at increasing difficulty, so only a significant influence of distraction on the percentage of correct hand positions was shown for the secondary task with the highest difficulty compared with the performance in tasks without distraction.

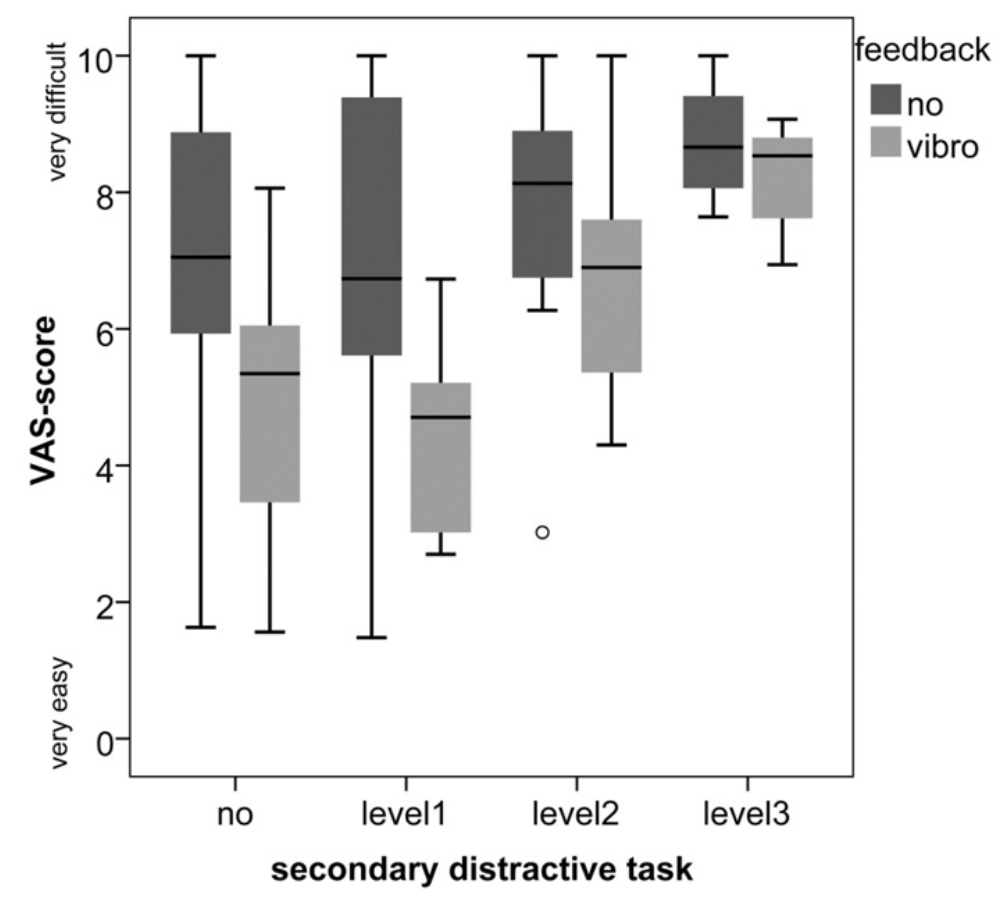

Figure 4: Distribution of visual-analog scale (VAS) scores for perceived difficulty of tasks per distraction level and feedback type. Horizontal bars represent median value, outer sides of boxes represent 25 and 75 percentiles, and whiskers represent minimum and maximum values. Small circles represent outliers with values larger than 1.5 times interquartile range. Vibro $=$ vibrotactile

Figure 4 shows the perceived difficulty for the different levels of distraction and type of feedback provided. A large range for the perceived difficulty for the non-feedback condition when no secondary task was used can be seen. Almost the whole range of the VAS is used for this specific condition, showing a large variation in perceived difficulty over the subjects. We analyzed the influence of the distractive secondary tasks and the provided feedback on the perceived difficulty via repeated-measures ANOVA (Table 3). We saw a significant influence for both the type of feedback provided and the secondary distraction tasks. When no feedback was given through vibrotactile stimulation, subjects perceived the tasks to be more difficult than in the feedback condition. Feedback helped the subjects to perform the grasping task. To evaluate the effects of the different levels of 
distraction on the perceived difficulty, we performed post-hoc pairwise comparison of the VAS scores by Student t-tests. We performed six pairwise tests for each feedback condition and therefore used Bonferroni correction for multiple comparisons. We found no differences in VAS scores for the non-feedback conditions; all tasks were perceived to be equally difficult. For the vibrotactile feedback conditions, subjects perceived that tasks performed with level 2 or 3 distraction were more difficult than tasks without distraction ( $p=0.01$ and $p<0.001$, respectively), while we found no significant difference in perceived difficulty between experimental conditions with no distraction and with level 1 distraction.

Table 3: Analysis of variance (ANOVA) table for comparison of VAS scores for perceived difficulty. Factors in analysis were feedback type and distraction

\begin{tabular}{|l|c|c|c|}
\hline \multicolumn{1}{|c|}{ ANOVA } & df & F & p-value \\
\hline Feedback & 1 & 6.19 & $0.04^{*}$ \\
\hline Distraction & 3 & 13.53 & $0.00^{*}$ \\
\hline Feedback•Distraction & 3 & 1.94 & 0.15 \\
\hline
\end{tabular}

\section{Discussion}

The number of publications on sensory feedback for users of myoelectric forearm prostheses has been increasing over the last few years, confirming the need for these applications. However, the focus is mostly on force feedback, while hand aperture feedback is also required. In this study, we described a new approach using an array of eight vibrotactile stimulators that has been shown to increase the performance in a grasping task. Furthermore, this study provides an evaluation of the usability of the feedback in real-life situations by investigating the influence of secondary tasks on the performance.

\section{Effect of vibrotactile feedback}

We compared the performance of the subjects in the grasping tasks with the help of vibrotactile feedback and the non-feedback situations, expressed in the four outcome parameters. The results of this study showed that feedback about hand aperture through vibrotactile stimulation on the forearm significantly increased the percentage of correct hand positions and reduced the mean deviation from the correct hand position. However, the addition of feedback also significantly increased the time needed to complete the grasping tasks. These results correspond with the results of Kuchenbecker et al., Stepp and Matsuoka, and Wheeler et al., who also showed increases in their performance parameters at the cost of an increase in duration of the tasks $[83,140$, 155]. Note that the feedback modalities and stimulation methods they used are not comparable with those used in this study.

The increase in duration found in this study was $4.5 \mathrm{~s}$ on average for the whole task of grasping 40 objects, which is $0.11 \mathrm{~s}$ per object. It can be questioned and should be further investigated whether this increase really impedes the grasping movement.

We also evaluated subjective difficulty ratings for the experimental conditions with vibrotactile feedback and the non-feedback conditions. Tasks in which no feedback was 
provided (also no visual feedback) were perceived significantly more difficult than tasks in which subjects received the hand aperture feedback by vibrotactile stimulation. These results also coincide with the results of Stepp and Matsuoka [140]. However, the spread in difficulty ratings was extremely large for the non-feedback conditions. A subset of the subjects rated the non-feedback experimental conditions as difficult, probably because they felt uncertain about their performance, while the other subjects perceived the task as easy because they did not have to pay attention to the feedback and there was no punishment for bad performance.

\section{Effect of distraction and levels of distraction applied}

Evaluation of the difficulty ratings showed a clear increase in perceived difficulty when the difficulty level was increased. Three levels of distraction (none and levels 2 and 3) could be separated from each other by differences in perceived difficulty. We found no differences in perceived difficulty between the experimental conditions without any auditory stimuli and conditions in which the auditory stimuli had to be ignored by the subjects. This showed that the auditory stimuli could indeed be ignored and did not interfere with the cognitive primary task. Every distraction is therefore related to the counting tasks and not to the presence of auditory stimuli. The previously mentioned results were only applicable for the vibrotactile feedback conditions. In the nonfeedback conditions, we found no significant differences in the perceived difficulty for the different distraction tasks. This is likely due to the extremely large spread in perceived difficulty over the subjects.

A trend in decreasing performance with increasing difficulty of the secondary tasks was shown in Figure 3. However, the percentage of correct hand positions significantly worsens only for the level 3 distraction compared with the tasks performed without any secondary tasks. The other performance parameters were not significantly influenced by the secondary tasks. This is largely comparable with the results found by Stepp and Matsuoka, who showed an increase in trial times but no significant decrease in performance [140]. In their study, they used only one level of a distractive cognitive secondary task and force feedback method through one stimulator, which cannot be compared with the tasks used in our experiment. The increase in trial times is also shown in our results, indicating that the secondary tasks were attention-demanding. In our experiment, a resting period was incorporated in the tasks, during which the hand had to be kept in the perceived correct position for $2 \mathrm{~s}$. Auditory stimuli were presented randomly over the whole duration of the task and therefore also occurred during the resting period, which did not influence the duration of the task. The influence of distraction on the task duration, therefore, can be even larger.

\section{Methodological considerations}

We used a virtual environment to simulate the opening and closing of a (prosthetic) hand because this enabled us to use nondisabled subjects instead of subjects from the small pool of patients with amputation. However, the results of this study should also be affirmed for this group of potential users. The normal pathways of hand aperture 
feedback in nondisabled subjects are blocked by the virtualization and the only feedback available is the visual and/or the vibrotactile feedback, which is highly comparable with the situation of a myoelectric prosthesis. The movement of the virtual hand is controlled by the scroll wheel of a computer mouse and therefore not comparable with the myoelectric control of today's prostheses. We did not use myoelectric control in this experiment because a long period of training is required and the differences between subjects in the ability to control the hand by electromyography were large, as was shown in preliminary experiments. The muscles used to control the scroll wheel are, to some extent, comparable with those used to control the hand aperture, and therefore the most intuitive way to control the virtual hand without a direct relation to the hand aperture. To remove clues providing information about the hand aperture other than the visual and vibrotactile ones, we removed the click mechanism from the mouse scroll wheel and the gain between the scroll wheel movement and randomized the hand movement. This approach has shown to be effective because the performance in the non-feedback experimental conditions was comparable with the results that can be expected for pure guessing. Scores of $16 \%$ were expected, but the scores were somewhat higher $(20 \%-25 \%)$ in our study because of the known end positions of the hand.

We chose the secondary auditory counting task to prevent structural interference with the primary task. However, a secondary task involving the measurement of reaction times is a somewhat more commonly used and accepted method $[72,160]$.

The VAS used to evaluate the perceived difficulty is a generally used method to evaluate subjective scores. However, some problems were encountered in our experiments for the most difficult tasks. When a task was perceived difficult, this was scored at the end of the scale, but another even more difficult task could not be scored higher on this scale. This resulted in difficulty scores clustered around the end point of the VAS. This phenomenon has been described more often in studies where VAS scores are used [85]. A VAS scale without fixed end points could have solved this problem [58] and probably more differences between VAS scores could be seen.

\section{Recommendations and practical implications}

The effect of training on the performance in the grasping tasks when feedback is provided should not be underestimated. Based on a recent study on the effect of training of tasks with vibrotactile force feedback, we expect that the performance in grasping tasks will improve significantly with training [139]. Furthermore, research on motor learning has demonstrated that the duration of tasks decreases and automation of task execution improves after training [68]. This means that feedback will be processed at a more subconscious level and, therefore, secondary tasks can be expected to have even less influence. While these aspects play an important role, it is most important to develop a method that requires the least amount of attention at the first use to avoid early rejection of the prosthesis. 


\section{Conclusions}

Although distraction influences the performance in a grasping task when feedback is provided, these effects were not significant for the first two levels of distraction. It can be concluded that hand aperture feedback through an array of vibrotactile stimulators is successful because it significantly improves the performance in grasping tasks while requiring minimal attention. However, the practical implications of this study for prosthesis users cannot be fully derived from this study and should be further investigated. 


\section{Chapter 4 - Vibrotactile grasping force and slip feedback}
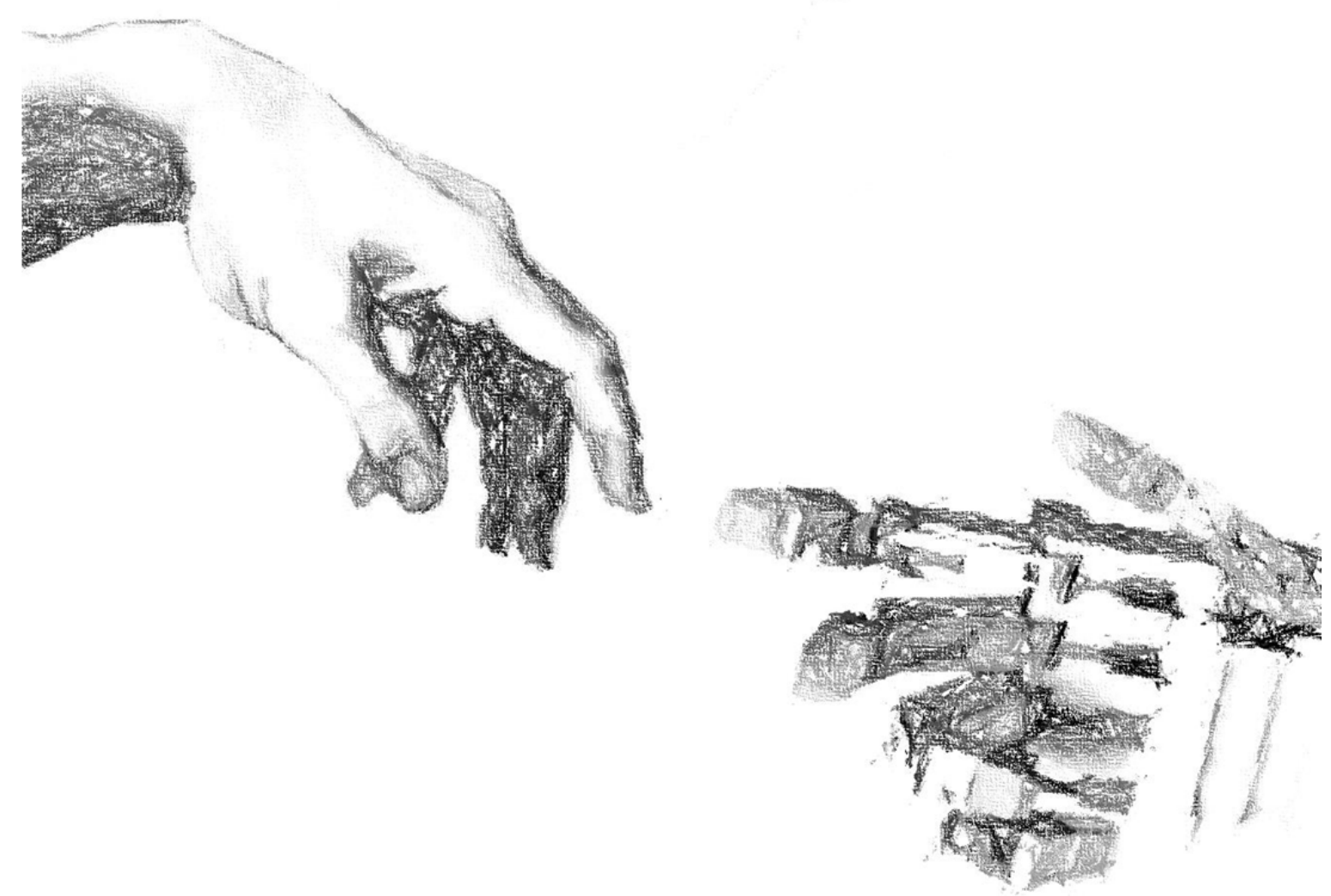

Published as: Grasping force and slip feedback through vibrotactile stimulation to be used in myoelectric forearm prostheses

Authors: Heidi Witteveen, Hans Rietman, Peter Veltink

In: Proceedings of the 2012 Annual International Conference of the IEEE

Engineering in Medicine and Biology Society (EMBC), pp. 2969-2972, 2012 
4 - Vibrotactile grasping force and slip feedback

\begin{abstract}
User feedback about grasping force or slip of objects is lacking in current myoelectric forearm prostheses, resulting in a high number of prosthesis abandonment, because a high level of concentration is required to hold an object. Several approaches to provide force feedback to the user via vibrotactile stimulation have been described in literature, but none of them have investigated the optimal stimulation parameters. This study describes an evaluation of three modulation techniques to provide force feedback. Furthermore, the same modulation techniques to provide slip feedback were evaluated, which has not been described before. The performance in virtual object holding tasks was significantly improved in most cases compared to the non-feedback situation, but at the cost of an increased task duration.
\end{abstract}




\section{Introduction}

Current myoelectric forearm prostheses offer an increasing level of functionality. However, the number of forearm prostheses being used on a daily basis remains low. One of the reasons of this prosthesis abandonment is the lack of user feedback about grasping force or occurring slip [12]. In situations without sensory feedback about grasping force, people tend to apply too much force to avoid slippage of objects [154], requiring more muscle activity than necessary. Furthermore, difficulties in handling of delicate objects will be experienced when no grasping force feedback is present. Early approaches to provide grasping force feedback to prosthesis users mainly focused on electrotactile stimulation $[16,115,127]$, providing force feedback through amplitude, pulse frequency or pulse width modulation respectively. Because the range between sensation and pain thresholds for electrotactile stimulation is rather small and due to recent miniaturization of vibrotactile stimulators, latest studies focus more on vibrotactile stimulation $[28,39$, $40,113,116,140]$. In most studies, a single stimulator (C2 tactor) is used and force is translated through frequency [40], pulse frequency [28, 39] or amplitude modulation [39, 140]. In two other studies, small coin motors were used to provide force feedback through frequency modulation $[113,116]$. The use of an array of these coin motors also shows some possibilities, but has only been used in one study on force feedback [123]. The outcomes of these studies are highly variable, ranging from no improvements compared to the non-feedback situations [28] to a measured reduction in muscle force needed to lift objects [116]. These variability is probably caused by the lack of a thorough evaluation of the possible stimulation methods that can be used. Only in one study a comparison is made between pulse frequency and amplitude modulation [141], but not in relation to an array of coin motors. In this study we have evaluated the performance of healthy subjects in a virtual object holding task, while force feedback was provided through vibrotactile stimulation. Amplitude and pulse frequency modulation via a C2 tactor and position modulation through an array of coin motors were used to provide the vibrotactile stimulation. Furthermore, we also evaluated these modulation techniques for feedback about slip of objects. Potentially, slip feedback can be more useful than grasping force feedback, because no preliminary information about the weight or surface of an object is needed.

\section{Methods}

\section{Subjects}

Measurements were performed on 15 healthy subjects $(26.4 \pm 2.4$ years; 6 male, 9 female), all students and staff of our department. All subjects did not have any experience with vibrotactile stimulation before and did not have any sensory or skin problems of their forearm. All were right-handed or at least control the computer mouse with their right hand. Subjects were informed about the study via an information letter and all signed informed consent before the start of the experiment. The study protocol has been approved by the local medical ethical committee (Medisch Ethische ToetsingsCommissie Twente). 


\section{Materials}

Vibrotactile stimulation was applied either through an array of 8 commercially available coin motors (Ineed, China) or a single C2 tactor (Engineering Acoustics, Inc., Casselberry, Florida, USA). The coin motors were chosen, because they already showed good results [116], are easy to use, small and low-priced. A rotating inner mass results in stimulation in a tangential direction to the skin. All 8 stimulators were activated with a driving current of $44 \mathrm{~mA}$, which was adjusted if necessary to create equally perceived amplitudes. The coin motors were driven by a custom build control unit and a National Instruments DAQ system (NI USB-6211), controlled by a Labview syntax. The C2 tactor has already been used in a wide range of military and biomedical applications. Stimulation is applied in a vertical direction to the skin. The stimulation frequency was set to $250 \mathrm{~Hz}$, because this is the resonance frequency of the C2 tactor and important mechanoreceptors in the skin, Pacinian corpuscles, are most sensitive to this frequency. The amplitude and pulse frequency of stimulation were controlled by another NI DAQ system and a Labview syntax. All stimulators were attached to the skin by double-sided adhesive rings (EEG Kleberinge, The Netherlands).

\section{Experimental setup}

To block the available sensory pathways of the healthy subjects, a virtual representation of a hand holding a cylindrical object was built in Labview (Labview Inc., 2009b, USA). The grasping force applied to the object was controlled by the subjects through the scroll wheel of a computer mouse. The 'clicks' from this mouse wheel were removed and a random varying gain between the level of scrolling and the grasping force was introduced to force the users to fully rely on grasping force feedback through the vibrotactile stimulation.

The weight of the displayed object is varied randomly and presented to the subjects via a color bar (see Figure 1). 8 different weights, corresponding to 8 feedback levels were used and the applied force is also classified to 8 discrete force levels. During the first two seconds of the training phase a thin horizontal bar supported the object. After these two seconds, the supporting bar was removed and the result of the applied force was displayed. When the applied force was not correct, the object was either dropped or squeezed and the same object was shown again with a maximum of 5 trials per object. In the experimental phase, the subject was asked to apply the presumed necessary force level as fast and accurate as possible, but the effect was not shown and after 4 seconds the next object was presented. The number of objects to be held in the training phase was 20 and 40 in the experimental phase 


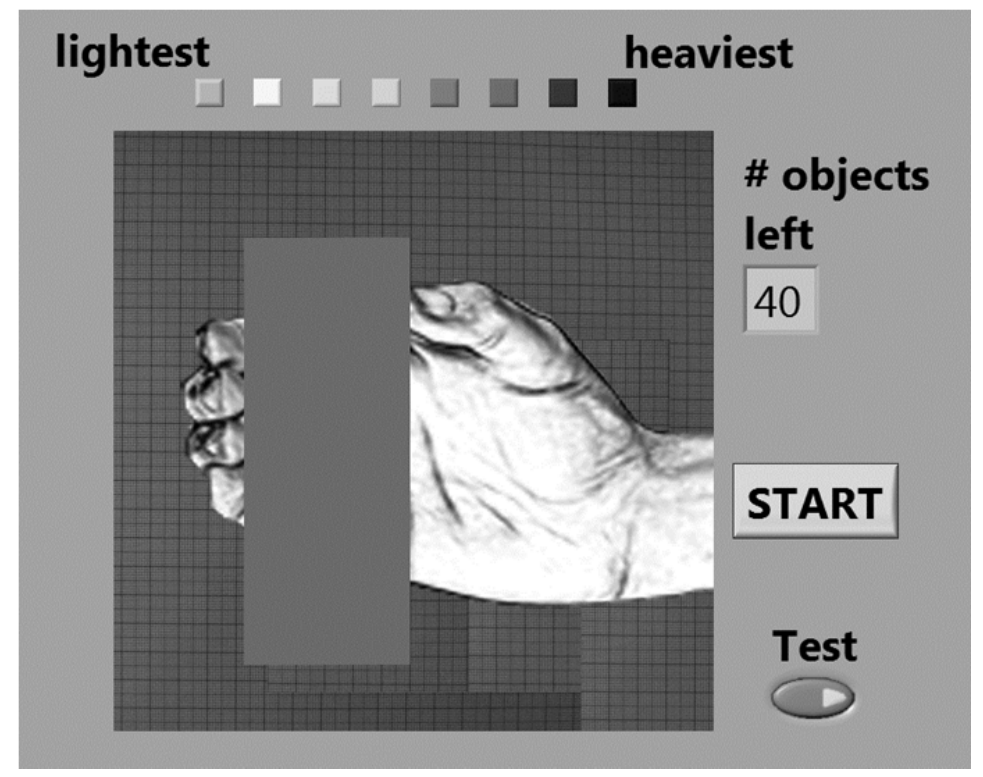

Figure 1: Virtual setup comprising a hand holding an object, which weight is color-coded

\section{Feedback}

Either feedback about the grasping force or slip was given. Level of slip in this study is defined as the level of movement of the object in relation to the hand, discretized in 8 levels. For grasping force feedback, the applied force was directly fed back to the subjects. Based on the visual weight information, the suitable grasping force must be determined. For slip feedback the task was to minimize the slip, by increasing the grasping force. During slip feedback, the visual representation of the weight of the object should not be necessary in the grasping tasks and is therefore blocked in half of the cases, by showing only white objects for every weight.

\section{Stimulation}

Three methods of stimulus modulation were used: (1) position (coin motors), (2) pulse frequency and (3) amplitude (C2 tactor) modulation. An array of 8 coin motors, placed around the thickest part of the forearm, was used to provide position modulation. Each force or slip level corresponded to activation of one of the coin motors. For amplitude and pulse frequency modulation an increase in force level or slip corresponded to a linear increase in amplitude or pulse frequency. The amplitude was varied between 1 and 4.5 Volts ( $0.5 \mathrm{~V}$ increase per force level) and pulse frequency between 4.35 and $50 \mathrm{~Hz}$, which corresponded to stimulus intervals of 230 to $20 \mathrm{msec}$. at a $50 \%$ duty cycle. The $\mathrm{C} 2$ tactor was placed at the dorsal side of the forearm, halfway between the elbow and wrist.

\section{Experimental conditions}

Both grasping force and slip feedback were provided for all three stimulation modulation methods. For all feedback options, a training session was applied before the measurement session. A control measurement (no vibrotactile and visual feedback), was performed between the change of stimulators and at the end of the experiment. No training was provided in the non-feedback situation, but the whole experimental setup 
was the same. For slip feedback, an extra measurement was performed without the visual weight information. The order of experimental conditions was randomized to avoid training effects.

\section{Outcome parameters and statistical analysis}

For the training phase, the number of attempts needed to reach the correct force level was determined and averaged over all objects. For the experimental phase, the applied force (discrete level) was compared to the required grasping force and based on this, the percentage correct force levels and the mean absolute deviation from the correct force level were determined. The task duration was calculated as the time needed to reach the final force level and summed over all 40 objects. ANOVA analyses and additional t-tests were performed to statistically evaluate the differences between stimulation parameters $(\mathrm{p}=0.05)$.

\section{Results}

\section{Descriptive statistics}

The mean absolute error between the defined and real grasping force level ranged between 0.3 and 0.61 for every combination of feedback and modulation method compared to 1.22 for non-feedback. The mean percentages correct force levels ranged from 54.2 to $75.7 \%$ compared to 32.8 to $65.6 \%$ for the non-feedback situations, which is higher than expected for pure guessing. The mean duration of the tasks was between 62.9 and $73.2 \mathrm{~s}$ and $50.7 \mathrm{~s}$ during non-feedback. The combination of slip feedback and pulse frequency modulation was left out from this evaluation, because this showed extreme deviations from the other values.

\section{Visual feedback}

Visual feedback about the weight of the objects was blocked in half of the cases of the slip feedback experiments. The effect on performance was evaluated via a pairedsamples t-test for every modulation technique and every outcome parameter. No significant differences ( $p$-values ranging from 0.08 to 0.96 ) were found. Therefore, the outcome parameters of slip feedback were averaged over both visual feedback conditions and used in further analysis.

\section{Feedback method}

A clear interaction effect $(p<0.001)$ between the type of feedback and the used modulation was found via ANOVA analysis of all parameters. Therefore, separate ANOVA analyses were performed for all modulation techniques.

Through the use of an array of coin motors (position modulation) the performance in the object holding task is significantly improved (see Figure $2 \mathrm{~b}$ ) compared to the nonfeedback situations (all p-values <0.001). However, the time needed to perform the tasks was also significantly increased (see Figure 2a) for both feedback methods in 
comparison to the non-feedback situation ( $\mathrm{p}=0.002$ and $<0.001$ respectively). all performance measures were equal for force and slip feedback. The number of attempts needed in the training phase was comparable for both feedback methods $(p=0.1)$.

Also for amplitude modulation the performance parameters were significantly higher for both feedback methods compared to no feedback $(p<0.001)$ and showed no differences between force and slip feedback.

Via pulse frequency modulation, slip feedback did not increase the performance in the experimental tasks compared to the non-feedback situation (p-values from 0.67 to 1). However, force feedback through pulse frequency modulation did increase all performance measures. The number of attempts needed to successfully perform the tasks was also significantly higher for slip feedback compared to force feedback $(p=0.004)$. Furthermore, also in these cases the duration of the tasks was significantly higher than in the non-feedback situation.

(a)

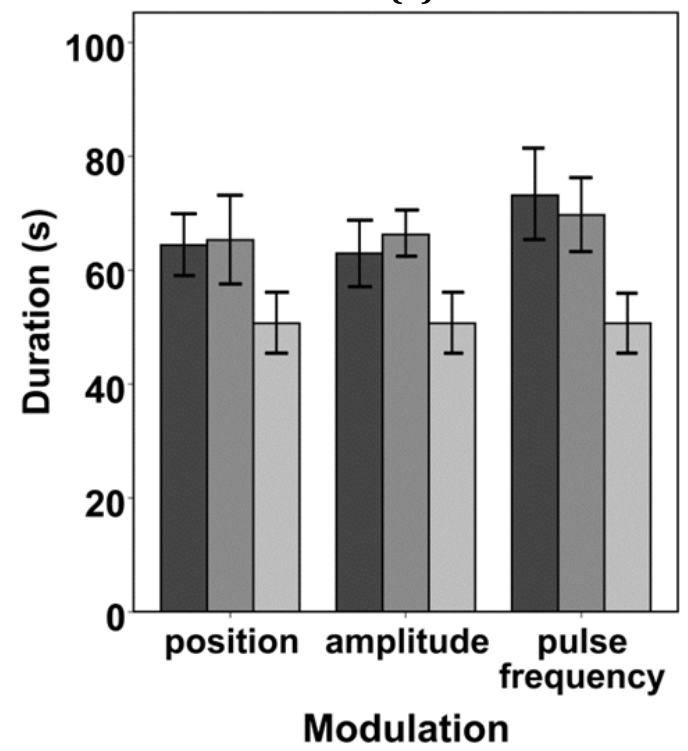

(b)

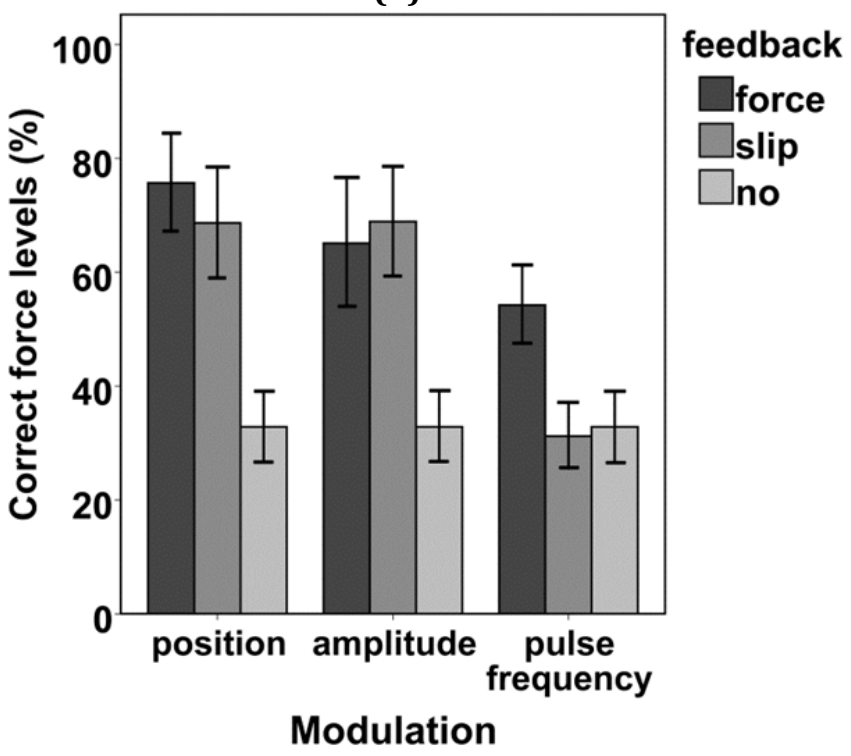

Figure 2: Mean and $95 \%$ c.i.'s of (a) the duration of the tasks and (b) the percentage correct force levels for each modulation method ( 1 method for the coin motors and 2 methods for the $\mathrm{C} 2$ tactor) and feedback method

\section{Stimulation modulation}

Due to the interaction effect between the type of feedback and the used modulation, separate ANOVA analyses were performed for both feedback methods.

In case of force feedback, no differences were found between the three modulation techniques for all three performance measures (p-values ranging from 0.05 to 0.80 ), except for the number of attempts in the training phase, which was significantly lower $(\mathrm{p}=0.001)$ for the position modulation in comparison to pulse frequency modulation (see Figure 3). Furthermore, the duration of the tasks was significantly higher when pulse frequency modulation was used $(\mathrm{p}=0.01$ and 0.03$)$ compared to the other two modulation techniques. 
In case of slip feedback, the performance parameters were highly comparable for position and amplitude modulation (all p-values were 1), while pulse frequency modulation showed significantly lower performances and a higher number of attempts in the training phase compared to the other modulation techniques $(p<0.001)$. No differences in duration were found between all three modulation techniques ( $p>0.5)$.

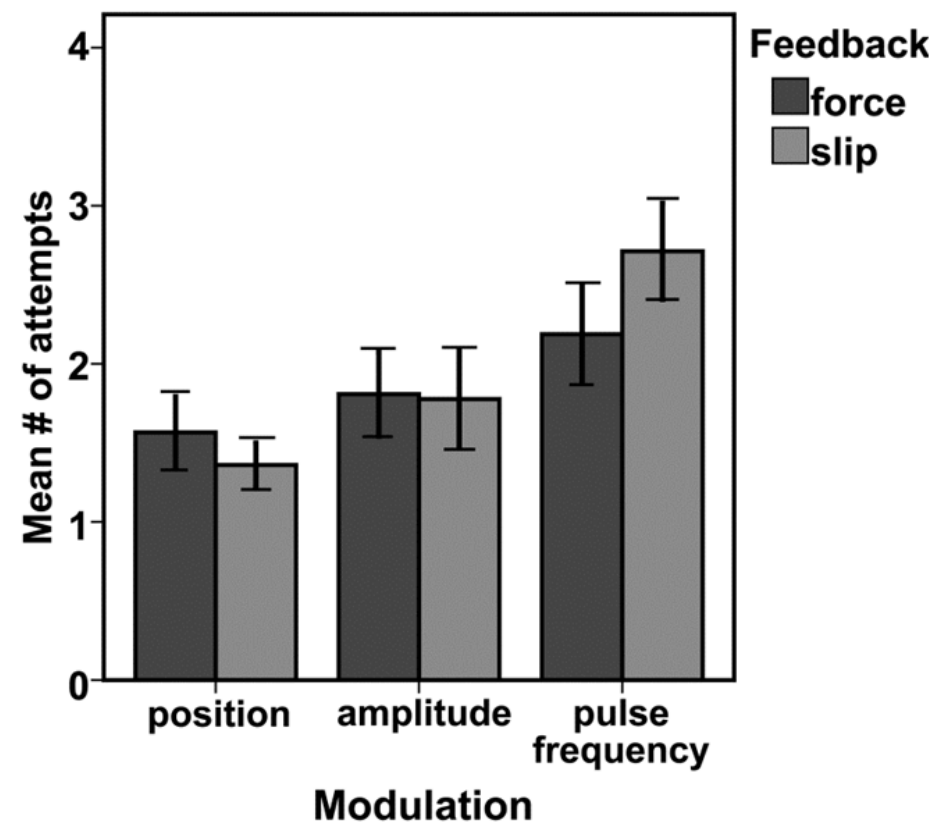

Figure 3: Mean and $95 \%$ c.i.'s of the number of attempts used in the training phase for each modulation technique and feedback method

\section{Discussion}

\section{Feedback method}

Although the performance without feedback was higher than expected, likely caused by the known endpoints of the force range, it is shown that the addition of artificial force feedback improves the performance, expressed in a significant decrease in absolute error and increase in percentage correct force, in a virtual object holding task. This improvement is not seen in every study on force feedback. Chatterjee et al. [28] used a C2 tactor to provide force feedback via pulse width and pulse frequency modulation, but found no improvement in distinguishing three force levels compared to the nonfeedback situation. We also experienced some problems with pulse frequency modulation, but only for the slip feedback situation, while the performance in force feedback with 8 levels was significantly better compared to the non-feedback situation. Pylatiuk et al. used a single coin motor to provide force feedback and they did show a reduction in applied forces by prosthesis users [116]. However, in another study, force feedback via frequency modulation of one coin motor was not shown to be successful [40]. They have improved this by the use of three coin motors on top of each other [39]. 
In our study we proposed the use of an array of coin motors to provide more feedback levels which shows to be successful. An improved performance together with an increased duration of the tasks was seen in the study of Stepp et al. [140], who used force feedback through amplitude modulation of a C2 tactor. This increase in duration of the tasks is also seen in our study. However, it is expected that the duration can be drastically reduced after periods of training with the feedback, which must be tested in future experiments.

Slip feedback to the user has not been described before. It has been incorporated already in several commercially available prostheses, but always to automatically control the grasping of the prosthesis. The advantage of the use of slip feedback instead of grasping force feedback is that there is no need for preliminary information about the weight or roughness of the object. Our results have shown that this visual weight information indeed is not necessary, because performance was not decreased when blocking the weight information on the screen. Furthermore, we showed no difference in performance compared to the force feedback method. These results are promising, but it should be further investigated, whether the slip can be detected and translated in different feedback levels, if it is possible to give feedback before the definite slip of the objects and if it is fast enough to react and change the grasping force.

\section{Stimulation modulation}

We have shown that better performances can be reached with position and amplitude modulation, especially for slip feedback. The performance with slip feedback through pulse frequency modulation was surprisingly low, which is likely caused by the hardly distinguishable lowest pulse frequency levels. A non-linear relation between slip and pulse frequency, with larger intervals between the lowest frequencies, can probably solve this issue. Problems with pulse frequency modulation were also seen by Chatterjee and Stepp et al., who used other pulse frequency levels [28, 141]. Amplitude modulation seems to be the most intuitive feedback method, because the number of attempts necessary in the training phase is lowest, especially for slip feedback where it performs significantly better than both other modulations. Furthermore, it seems more intuitive to provide force feedback through a single stimulator, because this is more related to the actual sense of force. Amplitude modulation would be the best option to provide force feedback, especially in combination with position feedback through position modulation.

\section{Methodological considerations}

We have used a virtual environment, consisting of a hand holding objects with different weights, instead of a real hand to block the normal sensory pathways of healthy subjects. Healthy subjects were chosen, because the number of amputee patients is rather small and this study was meant to be a first preliminary study to evaluate the stimulation parameters to provide slip or force feedback. Our findings, however, should be validated on prosthesis users. 
4 - Vibrotactile grasping force and slip feedback

\section{Conclusion}

It is shown that an array of coin motors as well as an amplitude modulated C2 tactor can successfully provide both force and slip feedback in a virtual grasping task. These results will be extended to real life grasping and evaluation on end users. 
Chapter 5 - Combined vibrotactile hand aperture and grasping force feedback

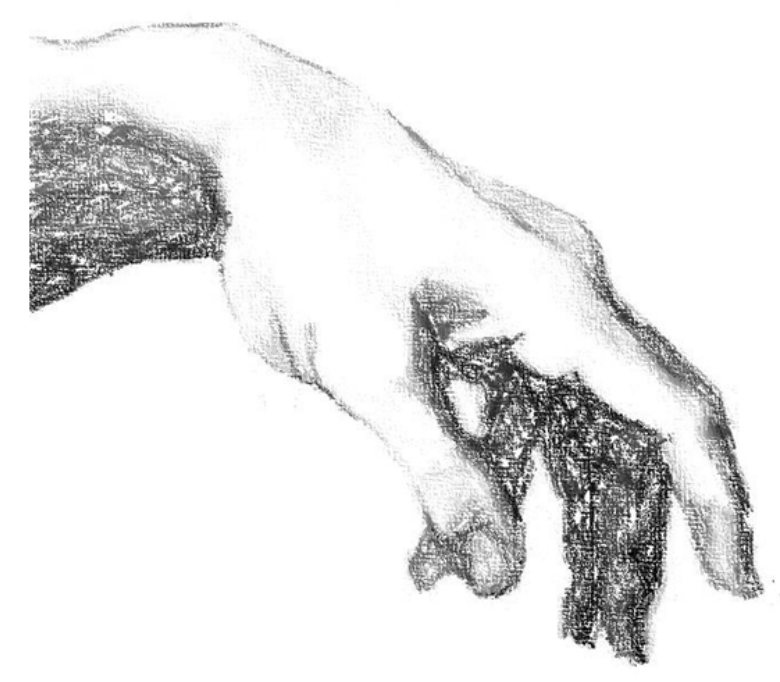

The combination of vibrotactile hand aperture and grasping force feedback Authors: Heidi Witteveen, Frauke Luft, Hans Rietman, Peter Veltink Submitted 


\section{Abstract}

Both grasping force and hand aperture feedback are essential for optimal control of myoelectric forearm prostheses. Vibrotactile stimulation is a non-invasive and relatively low-cost solution to provide this feedback. Several approaches have been undertaken to provide vibrotactile grasping force feedback, with varying results, but the number of studies on hand aperture feedback is low. Furthermore, it has not been investigated thoroughly how both feedback methods should be combined in one system. In this study the performance of healthy subjects in a virtual object grasping task is evaluated, while receiving hand aperture feedback through an array of 4 or 8 coin motors placed transversally or longitudinally on the forearm and grasping force feedback through amplitude modulation of a C2 tactor. Percentages correct grasping inputs were significantly improved with vibrotactile feedback, but the task duration was also significantly increased. The control configuration with hand aperture feedback through a C2 tactor and grasping force feedback through a transversal array of 6 coin motors showed the best performance. The combination of vibrotactile hand aperture and grasping force feedback successfully increased grasping performance, but these results should be evaluated in daily life grasping tasks with amputee patients using their myoelectric prosthesis. 


\section{Introduction}

Several studies have indicated the need for sensory feedback provided to the users of myoelectric forearm prostheses. Sensory feedback is required in optimal object handling [75] and reduces the mental burden on the visual system, enabling more subconscious control of the prosthesis [143]. Therefore, the lack of sensory feedback likely is one of the major factors in prosthesis abandonment $[18,90]$. However, in current commercially available myoelectric forearm prostheses no feedback to the users is incorporated. Some prostheses, for example the SensorHand of Ottobock [3], the Bebionic hand of RSLSteeper [4] and the I-limb of Touch Bionics [5], include the automatic adjustment of grip force based on slip sensing in the fingertips. However, prosthesis users still want to have control of their prosthesis and receive information about its behavior, which improves the embodiment of the prosthesis [44]. Based on the results of a workshop with representative prosthesis users, requirements for future myoelectric forearm prostheses were set and it was concluded that feedback about grasping force and hand aperture should be provided to the prosthesis users [107]. Although the hand aperture of a prosthesis can be seen, it is indicated as an important aspect to be fed back to the user in situations where visual feedback is not available and to diminish the visual attention required to control the prosthesis.

Several approaches to provide artificial sensory feedback to the users have been described in literature. Early approaches have focused on electrotactile stimulation, using amplitude, pulse rate or frequency modulation to provide the users with information about the grasping force between the thumb and first finger [16, 115, 127]. However, these methods often show some problems with the small stimulation range between the sensation and pain threshold [78]. Due to these problems and the possibilities of miniaturization recently, the focus has shifted more to vibrotactile stimulation.

Grasping force feedback through vibrotactile stimulation has been applied mostly by the use of a C2 tactor (a linear actuator), where the measured force at the fingertips is related to the amplitude [39, 140], frequency [40] or pulse frequency [28, 39] of stimulation. Besides the use of a C2 tactor, even smaller coin motors (rotating mass within a housing) have been used to provide grasping force feedback. In two studies the amplitude and frequency of stimulation were related to the grasping force $[113,116]$. However, the range of distinguishable stimulation levels is limited and therefore the use of an array of coin motors was evaluated [123]. The conclusions from these mentioned grasping force feedback studies range from no improvements compared to nonfeedback situations [28] to a decrease in grasping force during object holding [116]. Comparison of the performance in a virtual grasping task showed no differences between an array of coin motors and a C2 tactor providing grasping force feedback and both methods showed a significant increase in performance compared to the nonfeedback conditions [159].

Studies on hand aperture or position feedback are rarer in literature. In an early study hand aperture feedback was provided via pulse rate modulation of electrotactile stimulation [114] and in 1970, Mann et al. proposed the use of phantom sensations to 
provide feedback about the elbow angle of the Boston arm [93], which is an example of feedback that can possibly also be used for hand aperture feedback. An evaluation study was performed to compare the performance in a virtual grasping task with hand aperture feedback via electrotactile and vibrotactile stimulation provided through an array of eight stimulators, corresponding to discrete levels of hand aperture [157]. It was shown that both methods increase the performance in the grasping task compared to the non-feedback situation and that vibrotactile feedback was preferred over electrotactile feedback because of the longer task duration with electrotactile feedback. Although there is already a large number of research projects on grasping force feedback, none of the methods have been implemented in current prostheses. Among others, this can be caused by the lack of a proper investigation of the optimal parameters to provide the stimulation. As far as known by the authors, the combination of grasping force and hand aperture feedback has been investigated only in two studies. The use of electrotactile stimulation, where hand aperture was coded by the pulse rate of stimulation and grasping force by the amplitude of stimulation, was investigated by Prior et al. [114]. Their results showed that it was not possible to distinguish between the force and hand aperture feedback. By using four electrodes for grasping force feedback, discrimination between both feedback methods was slightly improved but not satisfactory [115]. The other study, by Chatterjee et al., focused on vibrotactile stimulation with a single $\mathrm{C} 2$ tactor [28]. The pulse interval of stimulation was related to the grasping force and the carrier frequency of the signal to the hand aperture. Only very experienced myoelectric forearm prosthesis users were able to use this feedback to increase the performance in grasping tasks with a myoelectric prosthesis.

This study aims to investigate a number of configurations to combine vibrotactile grasping force and hand aperture feedback. Hand aperture feedback is provided during object reaching and force feedback is added during object holding, where hand aperture is kept constant, simulating stiff objects.

\section{Methods}

\section{Subjects}

10 healthy subjects ( $26.8 \pm 10.2$ years, 5 male, 5 female) participated in this study. Subjects were included when they did not have any experience with vibrotactile stimulation and did not have any sensory or skin problems of the forearm. Subjects were all informed about the research via an information letter and all signed informed consent. The study protocol has been approved by the local medical ethical committee (Medisch Ethische ToetsingsCommissie Twente). All subjects were right-handed or at least used their right hand to control a computer mouse.

\section{Materials}

Two types of vibrotactile stimulators were used during the experiments: a C2 tactor and an array of coin motors. The C2 tactor (Engineering Acoustics Inc., Casselberry, FL, USA) is a disk shaped linear actuator $(3 \mathrm{~cm}$ diameter and $0.5 \mathrm{~cm}$ height), vibrating 
perpendicular to the skin with a passive housing which largely avoids spread of the vibration over the skin. The resonance frequency of the C2 tactor is $250 \mathrm{~Hz}$, which is the frequency at which important mechanoreceptors in the skin (Pacinian corpuscles), responsible for vibration sensing, are most sensitive. For the array, small coin motors (1 $\mathrm{cm}$ diameter, $0.3 \mathrm{~cm}$ height, Ineed, China) were used. The advantages of these coin motors over the $\mathrm{C} 2$ tactor are the size, weight and costs which make them more suitable for the use in an array on the forearm. The main disadvantage is that the frequency and amplitude of stimulation cannot be controlled separately and are influenced by the structure to which they are attached, which however is not a problem for use in an array.

(a)

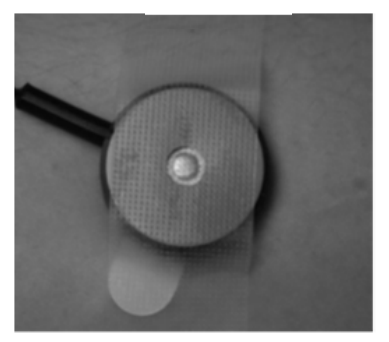

(b)

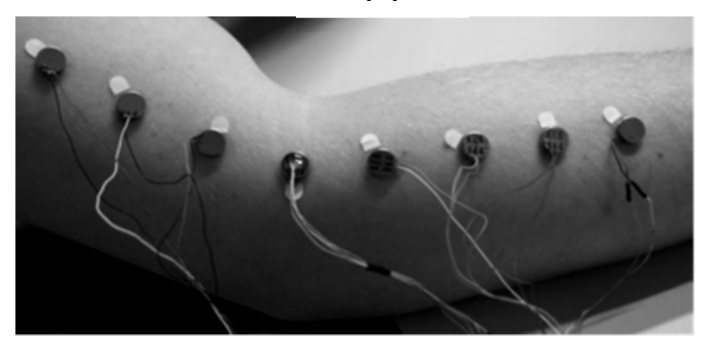

(c)

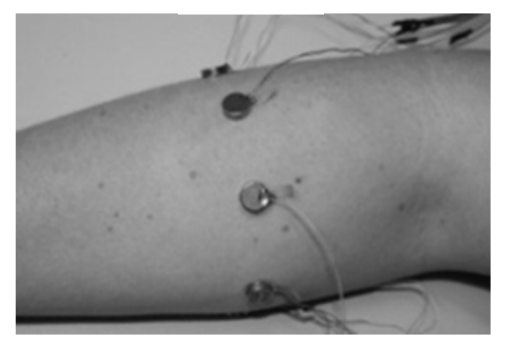

Figure 1: Pictures of the vibrotactile stimulators and their placement on the forearm, as used in the experiments. (a) C2 tactor secured with extra tape to ensure optimal contact with the skin. (b) longitudinal oriented array of 8 coin motors (c) transversal oriented array of coin motors around the forearm

\section{Grasping force feedback}

The C2 tactor was mainly used to provide the grasping force feedback, because in the human finger an increase in grasping force is also related to a local increase in sensation intensity. Six discrete amplitude levels of the $\mathrm{C} 2$ tactor, ranging from 0.8 to $3 \mathrm{~V}$ with equal intervals of $0.4 \mathrm{~V}$, were each related to an equal part of the force range. $\mathrm{A}$ normalized force range was used, ranging from 1 to 6 , whereby 6 stands for the maximal grasping force, resulting from maximal mouse scrolling. The C2 tactor (see Figure 1a) was placed at the ventral side of the forearm, $6 \mathrm{~cm}$ below the elbow, to minimize the interference with the array of coin motors. A custom-build stimulation device was used to control the amplitudes of stimulation. A stimulation frequency of $230 \mathrm{~Hz}$ was used.

\section{Hand aperture feedback}

Hand aperture feedback was mainly provided through an array of coin motors placed on the forearm (see Figure $1 \mathrm{~b} \& \mathrm{c}$ ). The range from a closed hand to a fully open hand is divided into eight discrete levels and each hand aperture level was related to the activation of one of the coin motors. Activation of the coin motors is controlled via a custom-build stimulation device. The driving voltage of each coin motor was kept constant at $2.5 \mathrm{~V}$, which resulted in clearly tangible sensations. The orientation of the array (transversal around the forearm or longitudinal from elbow to wrist) and the number of stimulators in the array (4 or 8 stimulators) were varied over the 
experiments. The stimulators were equally distributed around the arm, $3 \mathrm{~cm}$ below the elbow for the transversal orientation (see Figure 1c) and on the dorsal side of the forearm and upper arm for the longitudinal orientation of the array (see Figure 1b). The inter-stimulator distances for both array orientations were 2 and $4 \mathrm{~cm}$ for an array of 8 and 4 stimulators respectively. For the longitudinal configuration with 8 stimulators, 4 stimulators were placed at the forearm, 1 at the elbow and 3 at the upper arm. The array was placed across the elbow, because the space available on the stump of amputees will be limited. This placement across the elbow adds an extra bony landmark, which could increase the localization performance [35]. A dense array with 8 stimulators would provide extra hand aperture information while the less dense array with 4 stimulators will increase the localization performance, because the distance between the stimulators is larger than the discrimination threshold, which is maximally $4 \mathrm{~cm}$ for the forearm $[142,150]$.

We assumed that amplitude modulation of a single C2 stimulator would be best suitable for the grasping force feedback, because it is most similar to the localized sensation of pressure and assumed that an array of coin motors would be best for hand aperture feedback, because a change in position of the hand is most related to a change in position of active stimulators. To confirm this assumption, an extra, contradictory, configuration with grasping force feedback through an array of 6 coin motors (corresponding to the 6 amplitude levels of the $\mathrm{C} 2$ tactor) and hand aperture feedback through amplitude modulation (4 levels to be comparable with an array of 4 coin motors) of the C2 tactor was also evaluated. An overview of the vibrotactile feedback configurations is given in Table 1.

Table 1: Overview of the vibrotactile feedback configurations used during the experiments.

\begin{tabular}{|c|c|c|c|}
\hline $\begin{array}{c}\text { Hand aperture } \\
\text { feedback }\end{array}$ & \# stimulators & $\begin{array}{c}\text { Array } \\
\text { orientation }\end{array}$ & Name \\
\hline Coin motors & 4 & Longitudinal & $4 \mathrm{~L}$ \\
\hline Coin motors & 4 & Transversal & $4 \mathrm{~T}$ \\
\hline Coin motors & 8 & Longitudinal & $8 \mathrm{~L}$ \\
\hline Coin motors & 8 & Transversal & $8 \mathrm{~T}$ \\
\hline C2 tactor & - & - & C2 \\
\hline
\end{tabular}

\section{Experimental setup}

A virtual representation (see Figure 2) of a hand grasping and holding virtual objects was used to circumvent the intact sensory pathways of the healthy subjects. Circular objects with varying sizes and weights were simulated, requiring a certain hand aperture and grasping force to successfully hold the object. The hand aperture and grasping force of the virtual hand were controlled by scrolling a computer mouse wheel. Eight increasing levels of mouse scrolling were related to eight levels of hand aperture, ranging from a maximally open hand to a fully closed hand. When the object was touched, the mouse scrolling now controlled the grasping force. Six increasing levels of mouse scrolling were related to six discrete grasping force levels. Six different weights 
were randomly presented, corresponding to the six levels of grasping force that are needed to hold the object. The subjects were informed about the weight of the object by the color of the object and a color bar was presented above the simulated hand. The subjects were asked to grasp the object by applying the right hand aperture and hold the object by applying the right amount of force. Through mouse scrolling they first closed the hand, while receiving hand aperture feedback and when the object was touched, the mouse scrolling controlled the grasping force and grasping force feedback was provided. So, the final mouse scroll level is a combination of the applied hand aperture and grasping force. When subjects thought that they had reached the correct force level, they were asked to press the 'next' button and the grasping result was shown. If the grasping force was too high, the object was squeezed and if the force was too low, the object was dropped. Afterwards the next object was shown, always starting with a fully opened hand.

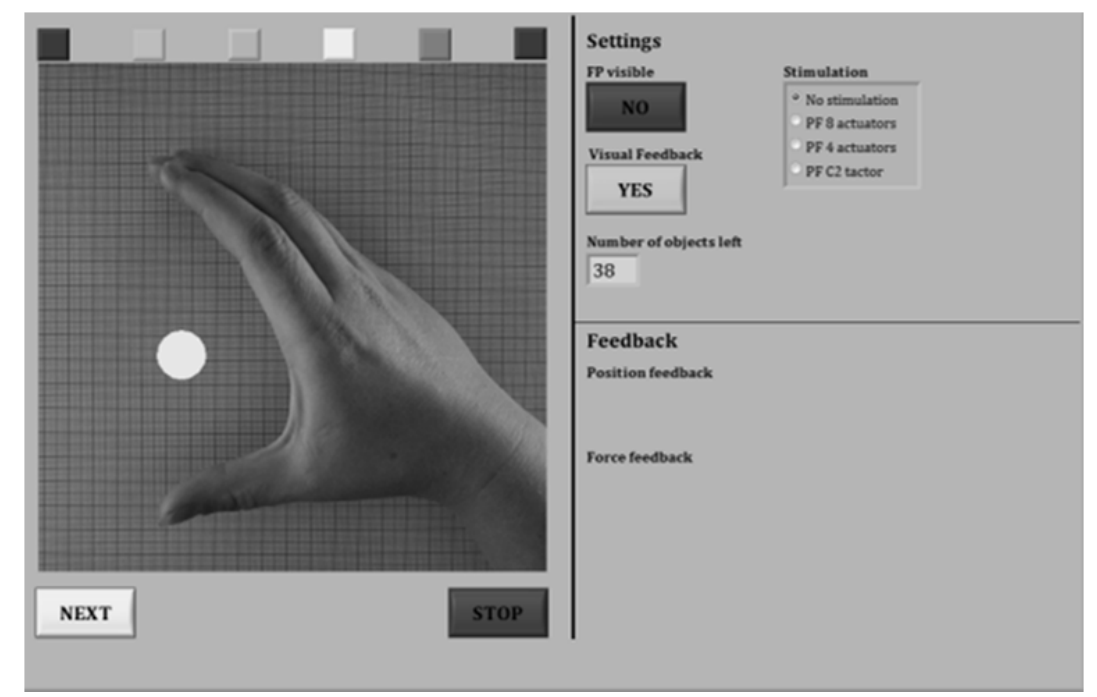

Figure 2: Experimental setup, showing the virtual representation of a grasping hand and an object to grasp. A color bar relates the color to the weight of the object

\section{Feedback conditions}

The two feedback conditions were 'no visual feedback' and 'visual feedback', where visual feedback stands for visual information about the hand aperture (visible hand), but no visual feedback about the applied grasping force is provided. The combination of feedback conditions and configurations represented four possible situations with current and future prostheses and were all evaluated during the experiments (see Table 2). The availability of visual and vibrotactile feedback was varied per condition. The two vibrotactile feedback situations were evaluated for each vibrotactile feedback configuration and randomly provided during the experiment. Each grasping task (combination of configuration and feedback condition) consisted of 25 objects to be grasped.

Preceding each grasping task, a short training session was provided to give the subjects time to adjust to the new configuration and/or feedback condition. This training session consisted of the grasping of 15 objects during which the level of hand aperture and 
grasping force were visually represented (visible hand and vertical slide bar showing the applied force). The performance during training was not used in the analysis of the results.

Table 2: Overview of the feedback conditions used during the experiments and their representation in the daily life situation

\begin{tabular}{|c|c|c|l|}
\hline $\begin{array}{c}\text { Feedback } \\
\text { situation }\end{array}$ & $\begin{array}{c}\text { Visual } \\
\text { feedback }\end{array}$ & $\begin{array}{c}\text { Vibrotactile } \\
\text { feedback }\end{array}$ & Prosthesis situation \\
\hline 1 & $\sqrt{ }$ & $\mathrm{x}$ & Current prosthesis \\
\hline 2 & $\mathrm{x}$ & $\mathrm{x}$ & Current prosthesis, blocked vision \\
\hline 3 & $\sqrt{ }$ & $\sqrt{ }$ & Future prosthesis with feedback \\
\hline 4 & $\mathrm{x}$ & $\sqrt{ }$ & Future prosthesis, blocked vision \\
\hline
\end{tabular}

\section{Outcome parameters and statistical analysis}

Whether an object was grasped and held correctly depended on the combination of successful reaching the object and the applied grasping force. Both were controlled via the mouse scrolling, so the required level of mouse scrolling was compared to the final applied level of mouse scrolling to derive the performance in the grasping tasks. Based on this comparison the percentages correct grasping inputs were calculated together with the absolute deviations from the correct grasping input (expressed in levels of mouse scrolling). Furthermore, the time needed to reach the final grasping input was determined. All parameters were calculated for each combination of configuration and feedback condition.

The no feedback data (with and without visual feedback) was subtracted from the data of all vibrotactile feedback configurations. Differences in outcome parameters between the vibrotactile feedback configurations and feedback conditions were evaluated via a repeated measures ANOVA test by using a general linear model in SPSS (IBM SPSS statistics 20). The feedback configuration and feedback condition were introduced as factor in this model with 5 and 2 levels respectively. If necessary, post-hoc Bonferroni corrected t-tests were performed to evaluate differences between feedback configurations. Differences from the no feedback condition were determined by evaluation of the intercepts of the parameter estimates from the general linear model. In case of no between-subject factors (which is the case for this data), these intercepts represent the marginal means of the data and therefore intercepts that were significant different from zero represent data that is significantly different from the no feedback situation. The significance level for all statistical tests was 0.05 .

Subject preferences for the used configurations were evaluated through a short questionnaire. After each task, subjects were asked to score the perceived usability, intuitiveness and comfort of the feedback configuration on a 5-point Likert-type questionnaire ranging from 'strongly disagree' to 'strongly agree' [47]. Average scores were calculated over the subjects for each configuration. Furthermore, subjects were also asked for each configuration whether they experienced the visual feedback as helpful over vibrotactile feedback alone. 
Subject preferences for the vibrotactile feedback configurations were evaluated through a short questionnaire. After each task, subjects were asked to score the perceived usability, intuitiveness and comfort of the feedback configuration on a 5-point Likerttype questionnaire ranging from 'strongly disagree' to 'strongly agree' [47]. Average scores were calculated over the subjects for each configuration. Furthermore, subjects were asked whether they perceived the visual feedback as helpful over vibrotactile feedback alone.

\section{Results}

\section{Descriptive statistics}

The level of mouse scrolling by the subjects resulted in a combination of hand aperture and grasping force and the final level of mouse scrolling, the grasping input, was the indicator for the performance in the grasping tasks. By comparison with the required level of mouse scrolling, the percentages correct grasping inputs were calculated and displayed in boxplots in Figure 3 for each vibrotactile feedback configuration and feedback condition. Vibrotactile feedback configurations were abbreviated according to the way hand aperture feedback was provided (see Table 1).

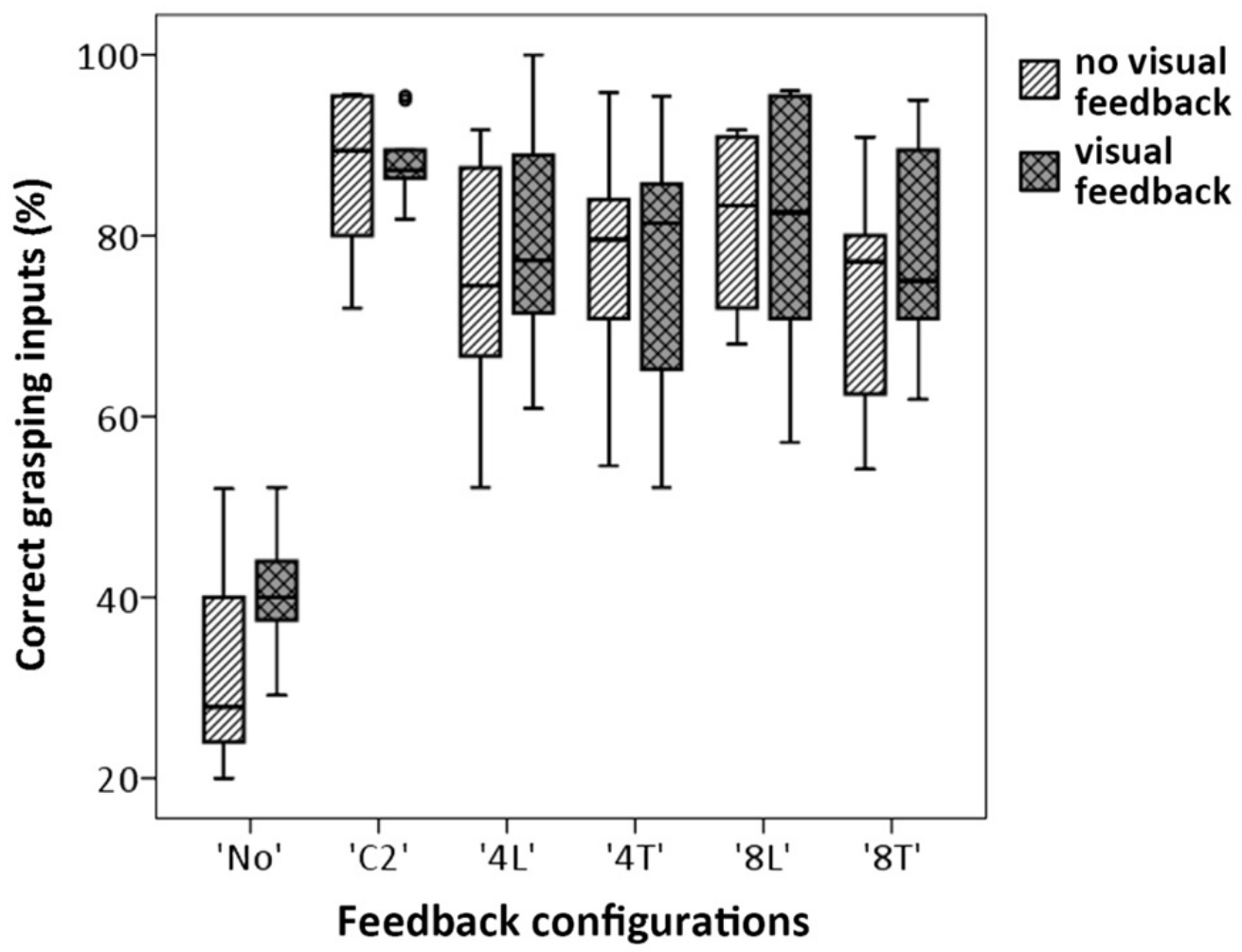

Figure 3: Percentages correct grasping inputs for each vibrotactile feedback configuration and both feedback conditions. Boxes represent the 25th and 75th percentiles of the data of all 10 subjects. Horizontal lines are the median values, the whiskers represent the minimum and maximum values and outliers are indicated by circles. Significant differences are indicated by asterisks 
In Figure 4 the task durations are shown for each vibrotactile feedback configuration and feedback condition.

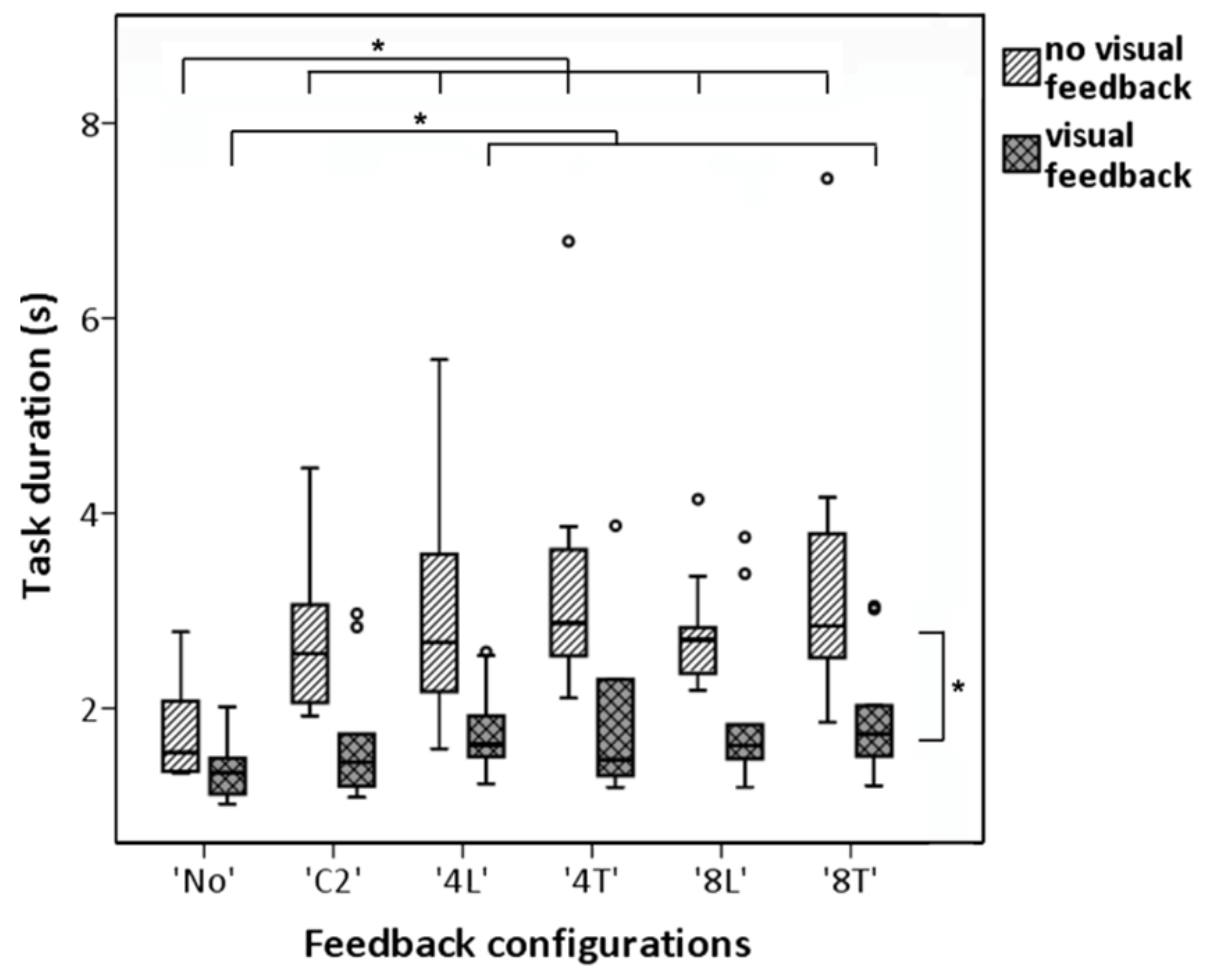

Figure 4: Task durations for each vibrotactile feedback configuration and both feedback conditions. Boxes represent the 25th and 75th percentiles of the data of all 10 subjects. Horizontal lines are the median values, whiskers represent the minimum and maximum values and outliers are indicated by circles. Significant differences are indicated by asterisks

\section{Grasping performance}

The repeated measures ANOVA revealed that there were no significant differences in percentages correct grasping inputs between both feedback conditions $(p=0.276)$. However, there was a significant influence of the vibrotactile feedback configuration on the percentages correct grasping inputs $(\mathrm{p}<0.001)$. Post-hoc paired samples t-tests showed that the only significant difference $(p=0.043)$ was found between the ' $C 2$ ' configuration and the '8T' configuration, showing higher percentages correct grasping inputs for the 'C2' configuration. All five vibrotactile feedback configurations showed significant higher grasping performances compared to the no feedback situation (all pvalues $<0.001$ ).

\section{Task durations}

A significant increase in task duration was found for the no visual feedback condition in comparison to the visual feedback condition $(\mathrm{p}=0.023)$, but no differences between feedback configurations were found $(p=0.052)$. Evaluation of the task durations of the separate vibrotactile feedback configurations compared to the task durations in the no feedback conditions, did show some differences between feedback configurations. The task durations for all vibrotactile feedback configurations without visual feedback were 
significantly increased compared to the no feedback condition ( $p$-values between 0.01 and 0.039). In combination with visual feedback, only the ' $4 \mathrm{~L}$ ' and the '8T' configuration did show a just significantly increased task duration compared to the no feedback condition ( $\mathrm{p}=0.04$ and 0.045 respectively).

\section{Questionnaire}

For all three subjective aspects (usability, intuitiveness and comfort) the scores on the 5point Likert-type questionnaire were derived. The number of observations per configuration was 10 , which is too low to perform a reliable statistical Chi-squared test for ordinal data. Figure 5 shows the mean scores on the 5-point Likert-type questionnaire and corresponding 95\% confidence intervals for all vibrotactile feedback configurations and the three subjective aspects. The mean usability and intuitiveness scores were between 3.4 and 4.2 for all vibrotactile feedback configurations. Wen no vibrotactile feedback was provided, the mean scores were 1.5 and 1.9 for usability and intuitiveness respectively. With mean scores between 3.9 and 4.6, the comfort was scored somewhat higher on the 5-point Likert-type questionnaire.

Visual feedback about hand aperture was indicated as extra helpful by all but one subject when no vibrotactile feedback was provided. For the vibrotactile feedback configurations with hand aperture feedback through coin motors, the opinions were split, but for the ' $\mathrm{C} 2$ configuration', 8 out of the 10 subjects did not experience the visual feedback as extra helpful in the grasping tasks.

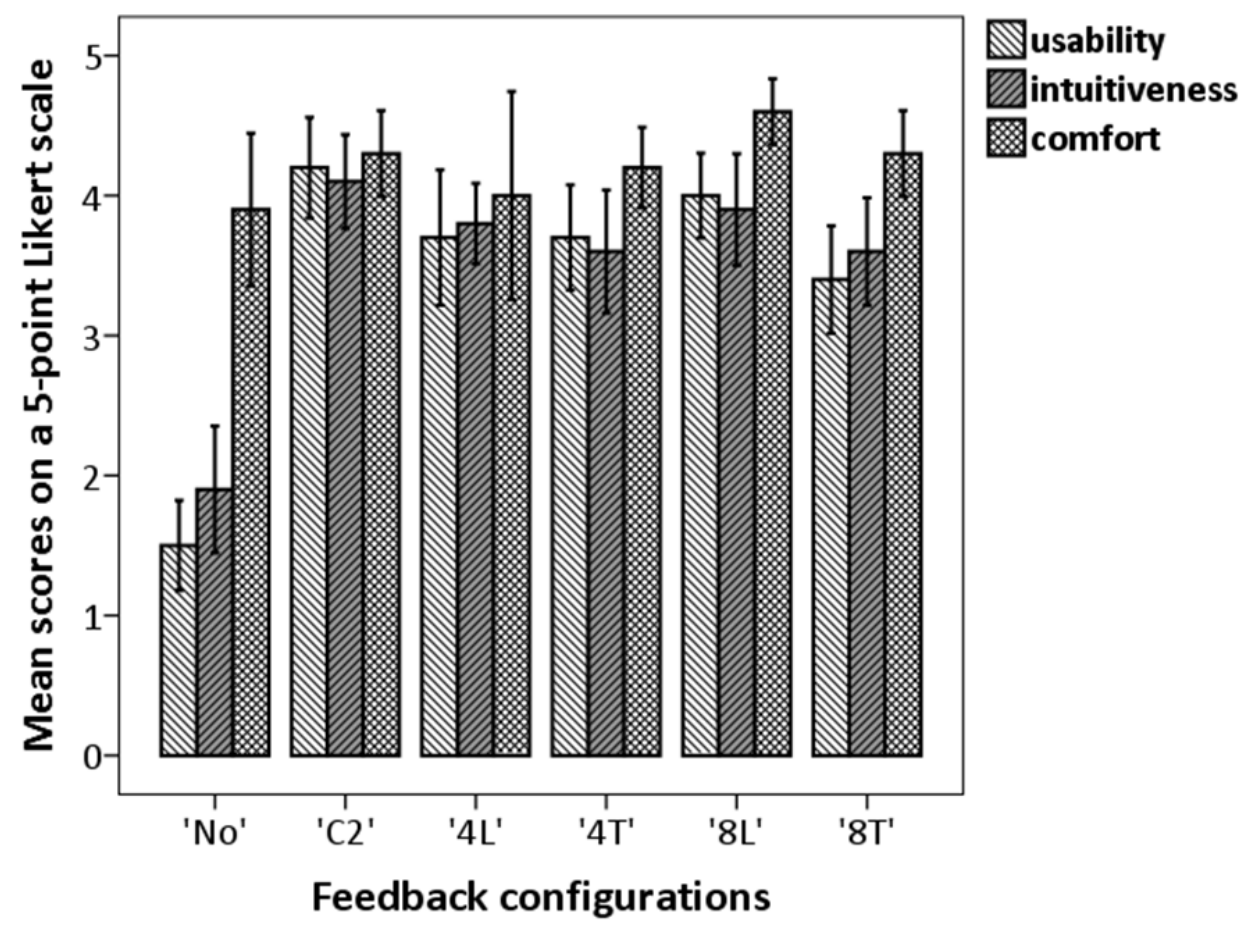

Figure 5: Mean scores and 95\% c.i.'s on the 5-point Likert-type questionnaire for every vibrotactile feedback configuration and all three subjective aspects 


\section{Discussion}

The performance in virtual grasping tasks with the help of vibrotactile feedback, as expressed in the percentage of correct grasping inputs, was significantly improved compared to the no vibrotactile feedback conditions. Furthermore, subjects also reported a high level of perceived comfort, usability and intuitiveness for the combination of vibrotactile hand aperture and grasping force feedback. However, the time needed to complete the grasping tasks with the help of vibrotactile feedback was also significantly increased. With percentages correct grasping inputs of around 80\%, the proposed combination of vibrotactile hand aperture and grasping force feedback is promising to be applied in future myoelectric forearm prostheses and likely to increase the grasping performance. However, it should be investigated whether the increased task duration is disturbing during daily life grasping tasks. The percentages correct grasping inputs were even higher than those found in recent studies on vibrotactile hand aperture and grasping force feedback [157, 159]. However, in these previous studies, an array of 8 coin motors or 8 amplitude levels of the C2 tactor were used, which reduces the localization performance. Furthermore, in the previous studies a random gain between the mouse scroll input and the resulting force or hand aperture was used, which excluded the feedback from the mouse scrolling, identifying the different levels, which also could have resulted in the lower performance values. However, the percentages correct grasping inputs, in the present study, were significantly higher with vibrotactile feedback than without, which proves the usefulness of the vibrotactile feedback over the mouse scroll feedback. Furthermore, the percentages correct grasping inputs in the no vibrotactile feedback situation are low and just above guessing level (16\%). They were somewhat higher than $16 \%$, because the lowest and highest force levels were easier to determine.

The combination of an array of coin motors and a single amplitude modulated C2 tactor to provide grasping force and hand aperture feedback, has not been described before, but the presented results show that this combination can be successful. Other studies $[29,115]$ on the combination of hand aperture and grasping force feedback showed that it was very hard to distinguish between both feedback modalities, likely because only one stimulator was used to provide both feedback modalities. No problems of interference between the $\mathrm{C} 2$ tactor and the array of coin motors were seen or reported by the subjects. However, hand aperture feedback was only actively provided during object grasping, while during object holding hand aperture feedback was kept constant. A combination of both feedback modalities during object holding, likely providing information about object stiffness, can possibly result in more problems in distinguishing the $\mathrm{C} 2$ tactor and the array of coin motors.

The addition of visual feedback about hand aperture did not further increase the performance in the grasping tasks compared to vibrotactile feedback alone, which suggests that a prosthesis with vibrotactile feedback can also be used in dark environments or when sight on the prosthesis is blocked. However, based on the results from this study we cannot directly conclude that vibrotactile hand aperture feedback is 
really of additional value in situations without visual feedback, because the task duration was significantly increased when using vibrotactile feedback.

The performance in grasping tasks was highest for the vibrotactile feedback configuration of hand aperture feedback through a single C2 tactor and grasping force feedback through an array of coin motors. This was even significant when comparing the $\mathrm{C} 2$ tactor configuration with the '8T' configuration, which was not expected beforehand. A preference for the $\mathrm{C} 2$ tactor to provide hand aperture feedback was also mentioned by the subjects who indicated that visual feedback was not of additional value for this configuration. In this study hand aperture feedback was not very important, because it was mainly used to give an indication of the direction of movement and the focus was more on the grasping force feedback. It is possible that position modulation (the use of an array of coin motors) was better interpretable than amplitude modulation (use of a C2 tactor) for grasping force feedback, resulting in better distinguishable force levels. Furthermore, stimulus adaptation is more likely to occur with amplitude modulation than with position modulation, possibly resulting in lower performances. However, a localized change in sensation, as with amplitude modulation, may be more related to the localized sense of touch and when hand aperture feedback becomes more important, this may be intuitively related to a position modulated feedback signal.

Overall no differences between longitudinally and transversally oriented arrays of coin motors were found in this study. Usually, better localization performances are found for transversal orientations $[41,102]$, likely due to the smaller radii of the receptive fields of the mechanoreceptors in the transversal direction. However, in this study the longitudinal array was placed over the elbow joint, which created an extra anatomical landmark, which could increase the localization performance as was shown in earlier studies [35]. Another explanation could be the distance between the coin motor array and the $\mathrm{C} 2$ tactor, which was $10 \mathrm{~cm}$ for the longitudinal and $6 \mathrm{~cm}$ for the transversal array. A smaller distance made it harder for the subjects to distinguish between both stimulators as was also indicated by some of the subjects. However, the distance between the stimulators in both cases was largely above the discrimination threshold (which is around $4 \mathrm{~cm}[142,150]$ ) for vibrotactile stimulation on the forearm. Both orientations can be applied in myoelectric forearm prostheses, but a transversal array would completely fit within in the prosthesis socket, while a longitudinal array is intuitively more related to the orientation of the muscles used for hand aperture. Which orientation works or combines best with the EMG electrodes used for prosthesis control should be determined in future studies

To investigate whether it is necessary to provide precise information about the hand aperture or if it is enough to give more global information about the direction of movement, performances were compared for configurations with 8 and 4 coin motors in an array. No differences in all outcome parameters were found between these configurations. However, as already stated before, grasping force feedback was indicated to be more important than hand aperture feedback in the grasping task. Hand aperture feedback likely becomes more important when both feedback types are provided simultaneously, which should be further investigated. If in these future studies 
no effect of the number of coin motors will be found, a preference for 4 coin motors would be obvious considering the limited space in a prosthesis socket.

This study has been performed on healthy subjects, while the ultimate application will be used by amputees or subjects with a congenital defect, who may have an altered stimulus perception. However, measurements on upper limbs loss subjects were not included, because this study was set up primarily to investigate the possibilities of combining hand aperture and grasping force feedback. Moreover, in a previous study on hand aperture feedback, no differences between healthy subjects and upper limb loss subjects were found in a largely comparable setup [157]. To rule out the sensory information about hand aperture and grasping force from the healthy hand, a virtual setup was used and the virtual hand was controlled by mouse scrolling. Myoelectric control was excluded from this protocol, because preliminary experiments showed that this would require a long training period, while the performance is also highly variable over the subjects. However, in future studies, it should be investigated whether EMG control influences the performance in grasping tasks, while vibrotactile feedback about hand aperture and grasping force feedback is provided simultaneously. Moreover, EMG control also increases the difficulty of control and therefore would require more attention from the subjects, which probably influences the feedback interpretation. Furthermore, possible interference between the EMG sensing and the vibrotactile stimulation should be investigated.

\section{Conclusion}

The combination of hand aperture and grasping force feedback through vibrotactile stimulation with two separate stimulation methods improves the grasping performance in a virtual grasping task, which should be validated in future studies in real-life tasks with EMG controlled myoelectric prostheses. 


\section{Chapter 6 - Vibrotactile stiffness feedback}

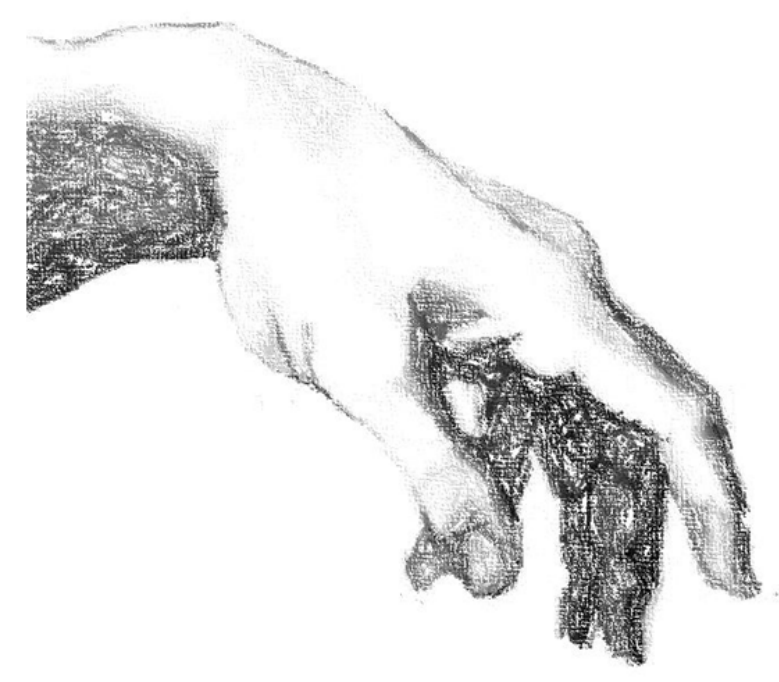

Published as: Stiffness feedback for myoelectric forearm prostheses using vibrotactile stimulation

Authors: Heidi Witteveen, Frauke Luft, Hans Rietman, Peter Veltink

In: IEEE Transactions on Neural Systems and Rehabilitation Engineering vol. 22, no. 1, pp. 1-9, 2014 


\section{Abstract}

The ability to distinguish object stiffness is a very important aspect in object handling, but completely lacking in current myoelectric prostheses. In human hands both tactile and proprioceptive sensory information is required for stiffness determination. Therefore, it was investigated whether it is possible to distinguish object stiffness with vibrotactile feedback of hand aperture and grasping force. Three configurations, consisting of an array of coin motors and a single miniature vibrotactile transducer, were investigated. Ten healthy subjects and seven subjects with upper limb loss due to amputation or congenital defects performed virtual grasping tasks, in which they controlled hand aperture and grasping force. They were asked to determine the stiffness of a grasped virtual object from four options. With hand aperture feedback alone or in combination with grasping force feedback, correct stiffness determination was achieved in around $60 \%$ of the cases and significantly higher than the $25 \%$ achieved without feedback or with grasping force feedback alone. Despite the equal performance results, the combination of hand aperture and grasping force feedback was preferred by the subjects over hand aperture feedback alone. No differences between feedback configurations and between subjects with upper limb loss and healthy subjects were found. 


\section{Introduction}

Artificial sensory feedback to the users of myoelectric forearm prostheses is not available in current commercial prostheses, but is indicated as one of the major improvements to increase user acceptance [18]. Based on the results of a workshop with representative myoelectric forearm prosthesis users, it was concluded that grasping force and hand aperture feedback are the most important feedback modalities to be applied in future prostheses [107]. Grasping force feedback is important in handling of delicate objects, while hand aperture feedback is important in situations where sight on the prosthesis is blocked. Furthermore, it is expected that this artificial sensory feedback will decrease the burden on the visual system and increase the integration of the prosthesis as part of the human body [44, 143]. Several approaches have been undertaken to provide non-invasive grasping force feedback, either through vibrotactile stimulation with a single C2 tactor (miniature vibrotactile transducer) [28, 40, 140], a smaller coin motor [113, 116], an array of coin motors [123], or electrotactile stimulation $[16,115,127]$. Fewer studies have focused on hand aperture feedback, using electrotactile stimulation [115] or arrays of coin motors or electrodes [157]. Most studies on hand aperture and grasping force feedback showed an increased performance in object handling compared to non-feedback situations. The combination of hand aperture and grasping force feedback has been investigated by only a few studies for electrotactile stimulation [115] as well as vibrotactile stimulation [29]. In both studies, one single stimulator was used to provide the combined feedback. For electrotactile feedback, pulse rate and amplitude modulation were combined [115] and for vibrotactile feedback, pulse interval and stimulation frequency modulation [29]. Both studies showed that it was hard to distinguish between both feedback modalities provided through one stimulator.

The combination of hand aperture and grasping force feedback during object handling likely provides information about the stiffness of the object, but has not been explicitly explored in the above mentioned studies. In the healthy human hand, both tactile (pressure at the fingertips) and kinesthetic (proprioception) information is needed to determine the stiffness of objects $[136,144]$. Visual information usually cannot provide enough information about object stiffness, because deformation is hard to observe visually or sight to the object can be blocked [136]. Stiffness discrimination is an important aspect in object handling, because it helps in object determination and prevents from damaging objects [136].

Only a few studies on object discrimination based on user feedback other than grasping force or hand aperture feedback alone could be found. In a study by Gurari et al. [64], subjects were asked to explore the stiffness of a virtual spring by exerting force with their index finger. Three methods to provide feedback about the deformation of the spring were evaluated: (1) visual feedback by showing the movement of a virtual finger, (2) proprioceptive feedback by passively moving the index finger and (3) the combination of both methods. They showed that equal performances could be derived with all three feedback methods, but Weber fractions (ratio between discrimination threshold and stimulus intensity) for object discrimination were lowest for 
proprioceptive feedback alone. In another study by Gurari et al. [65], surface discrimination was proposed by feedback of the vibration frequencies measured at the tip of a tele-operated manipulator while tapping foam surfaces with varying thickness and thus varying stiffness. Measured frequencies were directly related to stimulation frequencies of a $\mathrm{C} 2$ tactor attached to the foot, upper arm or fingertip of the subjects. They showed that it is possible to discriminate the three most diverse surfaces using the vibrotactile feedback, with similar results for the foot and the fingertip. In a study on two subjects with upper upper limb loss, by Horch et al. [71], nerves corresponding to the sensing of pressure at the fingertip and flexion of the index finger were intrafascicularly stimulated to provide the proprioceptive and tactile information needed for stiffness discrimination. When stimulating these nerves in one subject, this subject could correctly differentiate between four objects (2 sizes and 2 stiffness levels) in around $50 \%$ of the cases. However, the other subject could only be stimulated on two nerves both corresponding to tactile sensations and was not able at all to reach differentiation performances above chance level. Therefore, the authors concluded that both tactile and proprioceptive information is required for determination of stiffness.

None of the few described stiffness feedback approaches can be easily and noninvasively implemented in myoelectric forearm prostheses. Therefore, in our study we have investigated whether healthy subjects can determine the stiffness of virtually grasped objects when using a combination of vibrotactile stimulation methods, providing feedback about hand aperture and grasping force. We hypothesized that stiffness discrimination performance increases with the combination of vibrotactile hand aperture and grasping force feedback and further hypothesized that it is most intuitive to provide hand aperture feedback through an array of coin motors and grasping force feedback through amplitude modulation of a single C2 tactor, because hand aperture is most related to a change in position and grasping force to a local pressure sensation. To test these hypotheses, three configurations, combining both vibrotactile stimulation methods, were compared. Furthermore, it was investigated whether vibrotactile feedback about grasping force or hand aperture alone would also be sufficient for stiffness discrimination. The best scoring configurations were subsequently evaluated on subjects with upper limb loss due to amputation or congenital defects, who are the ultimate users of the feedback.

\section{Methods}

\section{Subjects}

Ten healthy subjects and seven subjects with upper limb loss participated in this study (see Table 1 for the characteristics). Ten subjects with upper limb loss were recruited via the local rehabilitation center, of whom three were excluded from this study. Two were not able to understand the instructions within the time limits of the experiment, due to language problems, and the third patient could not distinguish both stimulation methods, likely due to technical problems occurring during the experiment. None of the participating subjects reported severe sensory or skin problems of the forearm (stump). 
Furthermore, none of them participated in other studies on vibrotactile stimulation before. The seven included subjects with upper limb loss missed part of their forearm unilaterally due to traumatic amputation or congenital defects. The study protocol was approved by the local medical ethical committee (Medisch Ethische ToetsingsCommissie Twente) and all subjects signed informed consent.

Table 1: Subject characteristics. Mean values and corresponding standard deviations are given

\begin{tabular}{|l|l|l|l|l|l|l|}
\hline Subjects & $\#$ & Age (yrs.) & $\begin{array}{l}\text { Male/ } \\
\text { Female }\end{array}$ & $\begin{array}{l}\text { Forearm } \\
\text { length } \mathbf{( c m )}\end{array}$ & $\begin{array}{l}\text { Foreram } \\
\text { circumf. (cm) }\end{array}$ & $\begin{array}{l}\text { Amputation } \\
\text { / congenital }\end{array}$ \\
\hline Healthy & 10 & $22.9 \pm 1.7$ & $4 / 6$ & $25.9 \pm 2.2$ & $26.3 \pm 2.5$ & \\
\hline Limb loss & 7 & $54.1 \pm 10.5$ & $6 / 1$ & $14 \pm 4.8$ & $24.9 \pm 2.2$ & $5 / 2$ \\
\hline
\end{tabular}

\section{Materials}

Grasping force and hand aperture feedback were combined by using two distinct vibrotactile stimulation methods, namely position and amplitude modulation. For position modulation an array of small coin motors $(1 \mathrm{~cm}$ diameter, $0.3 \mathrm{~cm}$ height, iNEED (HK) Limited, Shenzhen, China) was used and activation of a single coin motor was related to a discrete level of hand aperture or grasping force. These stimulators were selected based on their small size and low costs. All coin motors were driven at a constant voltage of $3 \mathrm{~V}$, which resulted in clearly tangible sensations and stimulation frequencies between 50 and $120 \mathrm{~Hz}$ (depending on the underlying skin structure). These coin motors cannot be used for amplitude modulation, because the range of distinguishable amplitude levels is too low. Therefore, a C2 tactor (a miniature vibrotactile transducer) was used in addition. Discrete amplitude levels of the C2 tactor (3 cm diameter, $0.5 \mathrm{~cm}$ height, Engineering Acoustics Inc., Casselberry, FL, USA), with equal intervals between 0.8 and $3 \mathrm{~V}$, were used for feedback. The actuation frequency was kept constant at $230 \mathrm{~Hz}$, which is near the resonance frequency of the C2 tactor and within the optimal frequency range to activate the main mechanoreceptors (Pacinian corpuscles) in the skin. All vibrotactile stimulators were attached to the skin with double sided tape rings (EMG Kleberinge, GVB-geliMED KG, Bad Segeberg, Germany).

\section{Configurations}

Several configurations (varying the array orientation, the number of stimulators and the modulation technique) combining vibrotactile stimulation methods to provide feedback about hand aperture and grasping force were evaluated in a preliminary study (chapter 5), with an almost similar virtual setup. The three configurations resulting in the highest grasping performance and the lowest task duration were selected for this study. These three configurations were (see Figure 1): (1) hand aperture feedback provided through amplitude modulation ( 6 levels) of a C2 tactor placed at the ventral side of the forearm and grasping force feedback through an array of 6 coin motors placed transversally around the forearm (' $\mathrm{C} 2$ '); (2) hand aperture feedback through an array of 8 coin motors placed longitudinally on the dorsal side of the forearm, crossing the elbow, and grasping force feedback through amplitude modulation (6 levels) of the $\mathrm{C} 2$ tactor (' $8 \mathrm{~L}$ '); and (3) hand aperture feedback through an array of 4 coin motors placed longitudinally on the 
forearm, crossing the elbow, and grasping force feedback through amplitude modulation (6 levels) of the $\mathrm{C} 2$ tactor (' $4 \mathrm{~L}$ '). The inter-stimulator distances between the coin motors were 2 and $4 \mathrm{~cm}$ for the configurations with 8 and 4 coin motors respectively, which is around the spatial discrimination threshold found at the forearm [142] and equivalent to inter-stimulator distances as used in other studies [35, 157]. Further on, configurations will be referred to by the way hand aperture feedback is being provided (C2', '8L' and '4L').

(a)

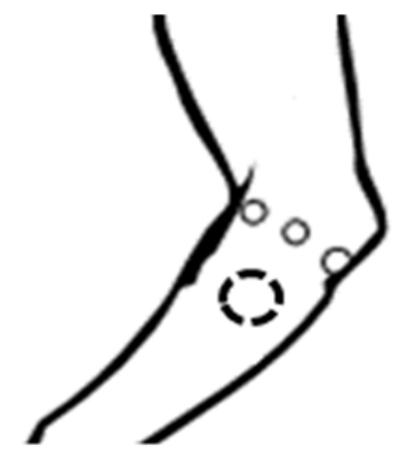

(b)

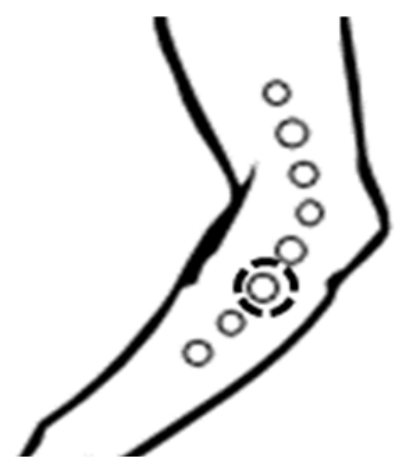

(c)

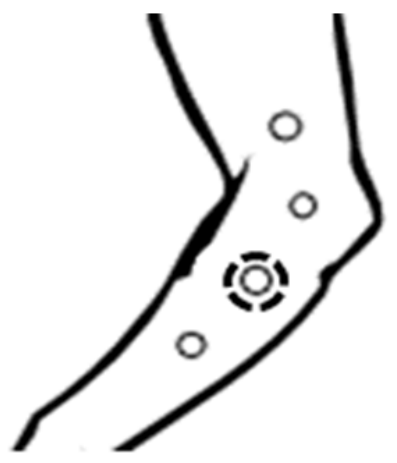

Figure 1: Overview of the three (a-c) vibrotactile actuator configurations, combining hand aperture and grasping force feedback: (a) providing hand aperture feedback via the $\mathrm{C} 2$ tactor (dashed line), which was placed at the ventral side of the forearm and grasping force feedback via 6 coin motors placed transversally around the forearm (called ' $\mathrm{C} 2$ '), (b) providing hand aperture feedback through 8 coin motors longitudinally oriented on the dorsal side of the forearm and grasping force feedback through the $\mathrm{C} 2$ tactor at the ventral side (called ' $8 \mathrm{~L}$ '), and (c) providing hand aperture feedback through 4 longitudinally oriented coin motors at the dorsal side and grasping force feedback through the $\mathrm{C} 2$ tactor at the ventral side of the forearm (' $4 \mathrm{~L}$ ').

\section{Experimental setup}

In Labview (Labview 2010 SP1, National Instruments, Austin, TX, USA) a virtual environment consisting of an opening and closing hand and circular objects was developed. The opening and closing of the hand was controlled via scrolling of a computer mouse. As soon as the displayed object was touched by the fingers, the level of scrolling became related to the level of applied grasping force. The gain between the mouse scrolling and the level of hand aperture and grasping force was randomized per object to avoid that subjects could use the mouse scroll as feedback. This was further eliminated by removal of the 'clicks' from the mouse wheel. Hand aperture and grasping force were discretized according to the available feedback levels with the vibrotactile stimulators ( 4 or 8 coin motors or 6 amplitude levels with the C2 tactor). Depending on the selected configuration, the range of hand aperture and grasping force was divided over the available stimulation levels.

Out of four stiffness levels, each presented circular object was randomly given a certain stiffness. Stiffness of an object was simulated by the level of object compression (change in hand aperture) with increasing grasping force (see Figure 2 for a graphical representation). For the lowest stiffness level or most compliant object, every discrete increase in grasping force resulted in a discrete decrease in hand aperture. For the 
second level of stiffness, each two steps in increasing grasping force resulted in the decrease of hand aperture of one level and for the third level of stiffness an increase of four steps in grasping force was needed to deform the object and decrease the hand aperture with one level. The stiffest object was not deformable and therefore the hand aperture did not change with increasing grasping force. Four different object sizes were simulated and randomly presented during the experiments. Only large objects, requiring a half-opened hand to a fully opened hand, were used, because for smaller objects not enough hand aperture levels would be available to provide object deformation information. The virtual hand was only visible during an introductory period and not shown during the experiments.

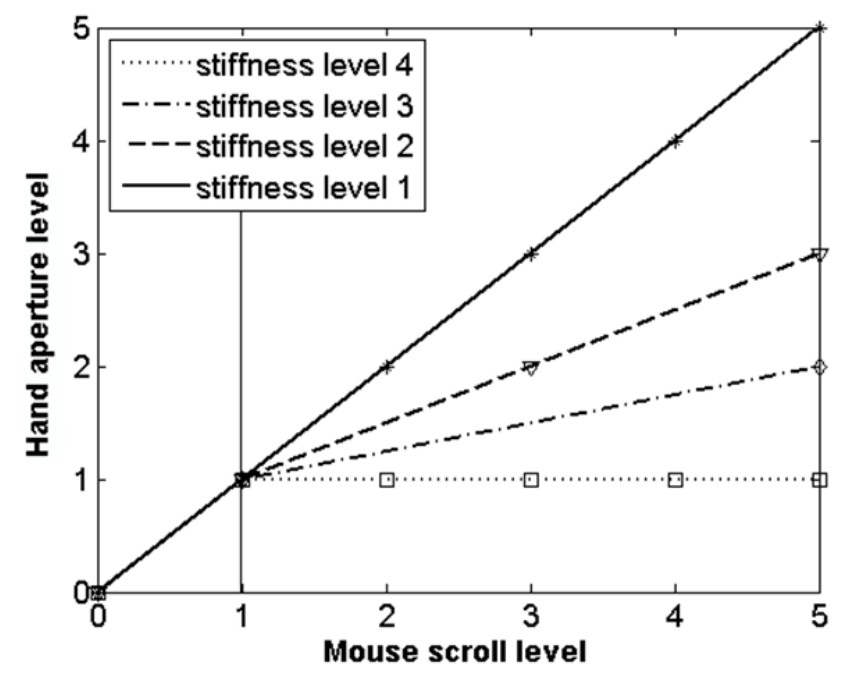

Figure 2: Graphical representation of the 4 different simulated stiffness levels. Mouse scroll first controls the hand aperture, but when the object is touched (vertical line), grasping force is controlled. The resulting change in hand aperture depends on the object stiffness

\section{Protocol for experiments with healthy subjects}

The goal for the subjects was to determine the stiffness of a presented object based on the given vibrotactile stimulation, providing feedback about the grasping force and/or hand aperture. An introductory period with spoken and visual instructions was provided first to introduce the feedback related to the four possible stiffness levels of an object. Next, a learning period was included during which 20 objects with randomly chosen sizes and stiffness were presented to the subjects and they had to select the perceived stiffness on the screen. After selection of stiffness, subjects were informed whether the chosen stiffness was correct, too high or too low. The results of the learning period were not used for statistical analysis. Subjects were not considered trained persons after this learning session, but had some time to get used to the vibrotactile stimulation and learned how to combine the hand aperture and grasping force information to determine the object stiffness. The learning period was only provided for the combination of hand aperture and grasping force feedback for each configuration. During the main experiment, feedback was provided through the combination of hand aperture and grasping force feedback, hand aperture feedback alone and grasping force 
feedback alone for each configuration. Furthermore, a condition without vibrotactile feedback was included. The order of the configurations and the provided feedback were randomized over the subjects. For each task 30 objects, randomly varying in size and stiffness, were presented. After each object the subjects were also informed whether the chosen stiffness was correct, too high or too low. During both the learning period and the main experiment, the subjects were allowed to scroll back and forward several times to determine the stiffness, but were encouraged to select the perceived stiffness as quickly as possible. When the subjects had finished the tasks for a given configuration and all three feedback methods, they were asked to fill in a short questionnaire. The usability, intuitiveness and comfort of the configuration were scored on a 5-point Likert scale [47]. For each topic a single score was given by selecting one of the five possibilities, ranging from not useful, not intuitive or not comfortable at all to very useful, very intuitive or very comfortable. Furthermore, they were asked which feedback method (hand aperture, grasping force feedback or the combination of both) they preferred.

\section{Outcome parameters and statistical analysis for healthy subjects}

The main outcome parameters were the percentage of correctly identified stiffness levels and the time taken by the subjects to identify the stiffness. For each subject and each parameter, the results were normalized to the no feedback situation by subtracting the no feedback data. A General Linear Model (GLM) in SPSS (IBM SPSS statistics 20) was used to perform repeated measures ANOVA to evaluate the performance differences between the varying feedback methods and configurations for each outcome parameter. The feedback method and feedback configuration were both included as factor in this analysis and the interaction effect between both factors was evaluated. To evaluate the differences from the no feedback situations, the intercepts of the parameter estimates resulting from the GLM were analyzed. Intercepts that were significantly different from zero, indicated a significant difference from the no feedback situation, because without between-subject factors, the intercepts represent the marginal means of the data.

Furthermore, the progress over time, indicating possible learning effects, was evaluated by comparing the performance in stiffness determination and the time needed to complete the task of the first half of the measurement with the last half. A stronger learning effect for a certain feedback method provides a preference for this method over the others. A repeated measures ANOVA (with feedback method and feedback configuration as factors with three levels each) was performed to statistically evaluate these differences. The significance level for all statistical tests was 0.05 .

\section{Measurements from subjects with upper limb loss}

To reduce the time required for the patient measurements, a selection of the protocol was made, based on the results from the measurements on healthy subjects. The two best scoring configurations, the no feedback condition and the best scoring feedback method were used. Subjects with upper limb loss controlled the virtual hand by mouse scrolling with their sound hand, while feedback was provided on the forearm stump. The 
C2 tactor was placed ventrally on the forearm, halfway the stump. Coin motors were only placed in the longitudinal orientation, because the distance between the transversal oriented coin motor array and the $\mathrm{C} 2$ tactor would be too small to be distinguished in case of a short stump. The inter-stimulator distance for the coin motors was $2 \mathrm{~cm}$ for each patient, which resulted in a variable number of stimulators that was placed on the upper arm.

The experimental setup was the same as used for the healthy subjects and the same steps were followed during the experiment: an explanatory session, a learning period and the experimental session. Performances in stiffness determination (normalized to the no feedback situation) were compared, via repeated measures ANOVA, between the different configurations and compared to the no feedback condition (by evaluation of the intercepts of the parameter estimates). The feedback configuration was included as factor with two levels. Furthermore, it was investigated, via unpaired samples t-tests, whether there were differences in performances or task durations between healthy subjects and subjects with upper limb loss.

\section{Results}

\section{Descriptive statistics}

(a)

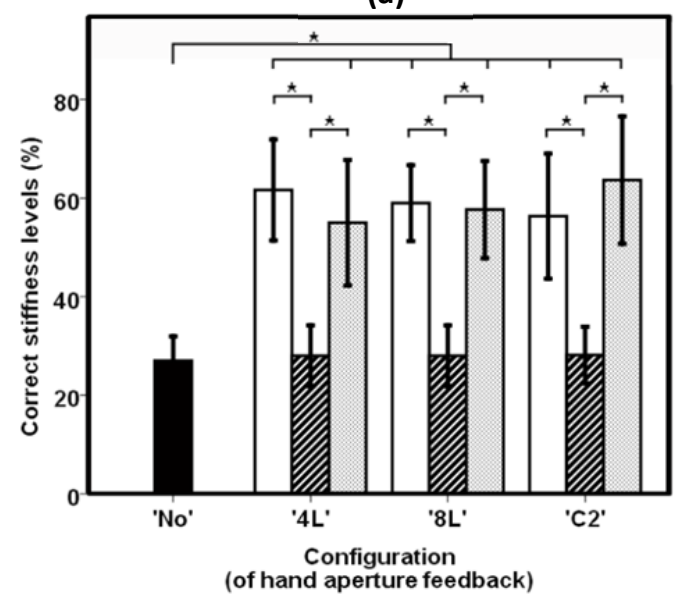

(b)

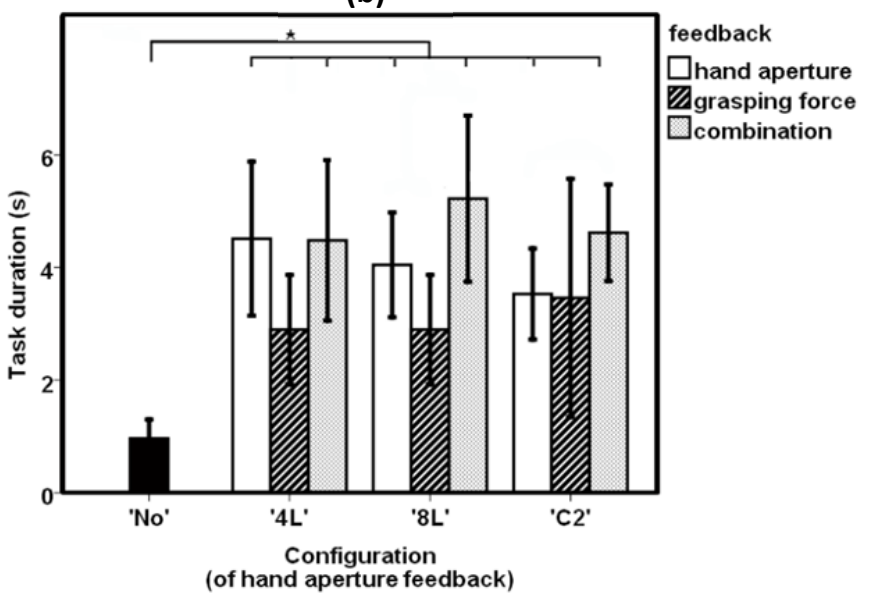

Figure 3: (a) Mean percentages correct stiffness levels and (b) mean task durations with corresponding $95 \%$ confidence intervals for the measurements on healthy subjects $(n=10)$. Results are categorized per feedback method (hand aperture, grasping force feedback or the combination of both) and per configuration (no feedback ('No'), hand aperture feedback through 4 ('4L') or 8 coin motors ('8L') or through a C2 tactor ('C2')). * indicates significant differences $(\mathbf{p}<0.05)$

Based on the comparison of the stiffness as indicated by the subjects and the actual stiffness of the displayed object, the percentage of correctly identified stiffness levels was calculated per task and averaged over all 10 subjects. Results are shown in Figure $3 a$, showing an average percentage of around $60 \%$ when vibrotactile feedback was given and around $25 \%$ for no feedback and grasping force feedback alone. The grasping force 
feedback alone condition was the same for the ' $4 \mathrm{~L}$ ' and the ' $8 \mathrm{~L}$ ' configurations, because no hand aperture feedback was given and the grasping force configuration was the same. It was, therefore, only measured once. Task duration was calculated as the average time needed to grasp one object. The mean and $95 \%$ confidence intervals of the task durations over all 10 subjects are shown in Figure $3 \mathrm{~b}$.

Normality of all data per configuration and feedback method was checked using a Shapiro-Wilk test, which showed that for the performance as well as the duration data a normal distribution could be assumed (all p-values above 0.05). Both outcome parameters were analyzed using repeated measures ANOVA with the configurations and feedback methods as factors in this analysis. The feedback method showed significant differences for the percentage correct stiffness levels as well as the task duration ( $p<0.001$ and $p=0.014$ respectively), but no interaction effects between both factors were found for all parameters.

\section{Feedback method}

To investigate the differences between the feedback methods, post-hoc Bonferroni corrected ( $p$-values times three for three tests) t-tests were performed for each combination of feedback methods. It was shown that the performance of stiffness determination was significantly lower for grasping force feedback alone compared to hand aperture feedback alone and compared to the combination of grasping force and hand aperture feedback $(\mathrm{p}<0.001$ and 0.002). No differences were found between the combination of feedback methods and hand aperture feedback alone (see Figure 3a).

In contrast to the main repeated measures ANOVA test, post-hoc testing did not reveal any differences in task duration between all three feedback methods (see Figure 3b). After Bonferroni correction, the p-values were just above significance level ( $p$-values $\geq$ 0.068).

\section{Learning effect}

The performance in stiffness determination did not significantly increase over time (mean increase over all configurations was $1 \%$ between the first and second half of the experiments) and no significant differences between feedback methods and configurations were found. The decrease in task duration between the first and second half of the experiments, averaged over all configurations, was 0.8 seconds and for every configuration a net decrease was seen. Two separate repeated measures ANOVA were performed: one with the feedback configurations resulting in stiffness determination performances around guessing level (no feedback and grasping force feedback alone) and one for the feedback configurations with results above guessing level (hand aperture feedback alone and the combination of hand aperture feedback and grasping force feedback). Both analyses showed a significant decrease in task duration for the second half of the experiment compared to the first ( $\mathrm{p}=0.046$ and $\mathrm{p}=0.004$ respectively). 


\section{Questionnaire}

The answers given by the subjects on the 5-point Likert scales were converted to values ranging from 1 for totally disagree and 5 for totally agree and were averaged over all 10 subjects (see Table 2). Moderate scores were found for the usability and the intuitiveness of the vibrotactile feedback, while scores for the comfort of stimulation were high. No statistical analysis was performed on this data, because the number of observations for each configuration was too low to perform a reliable test for ordinal data. Out of 10 subjects, 7 preferred the combination of feedback methods over hand aperture or grasping force feedback alone for the ' $8 \mathrm{~L}$ ' configuration. For the ' $\mathrm{C} 2$ ' configuration the number of subjects preferring the feedback combination was 6 against 3 preferring hand aperture feedback alone and 1 preferring grasping force alone. For the ' $4 \mathrm{~L}$ ' configuration the feedback preference was equally spread over the feedback combination and hand aperture feedback alone.

Table 2: Averaged scores and standard deviations on the 5-point Likert scales for usability, intuitiveness and comfort of the (vibrotactile) feedback. Scores are given for each feedback configuration separately

\begin{tabular}{|l|l|l|l|l|}
\hline Topic / Configuration & '4L' & '8L' & 'C2' & 'No' \\
\hline Usability & $3.5 \pm 1.1$ & $3.5 \pm 1.2$ & $3.4 \pm 1.3$ & $1 \pm 0$ \\
\hline Intuitiveness & $2.9 \pm 1.2$ & $3.1 \pm 1.2$ & $2.8 \pm 1.4$ & $1 \pm 0$ \\
\hline Comfort & $4.2 \pm 0.9$ & $4.1 \pm 1.0$ & $4.1 \pm 0.9$ & $3.3 \pm 1.6$ \\
\hline
\end{tabular}

\section{Measurements from subjects with upper limb loss}

From the results of the measurements with healthy subjects it was concluded that the combination of hand aperture and grasping force feedback and hand aperture feedback alone were the most successful methods to provide stiffness feedback. Thus, for the measurements on subjects with upper limb loss, the combination of hand aperture and grasping force feedback was selected as feedback method, because it was indicated by the subjects as being more useful than hand aperture alone, despite the slightly higher task duration for the combination. No significant differences in performance between the vibrotactile feedback configurations were found for the healthy subjects and therefore no selection based on performance could be made. The configurations with hand aperture feedback through the C2 tactor and hand aperture feedback via 4 coin motors were selected for the patient measurements, because the number of stimulators is lowest in these configurations, which is most favorable for the application in a prosthesis.

Averaged percentages correctly identified stiffness levels and task durations are presented in Figure 4 together with the averaged results from the healthy subjects. 
(a)

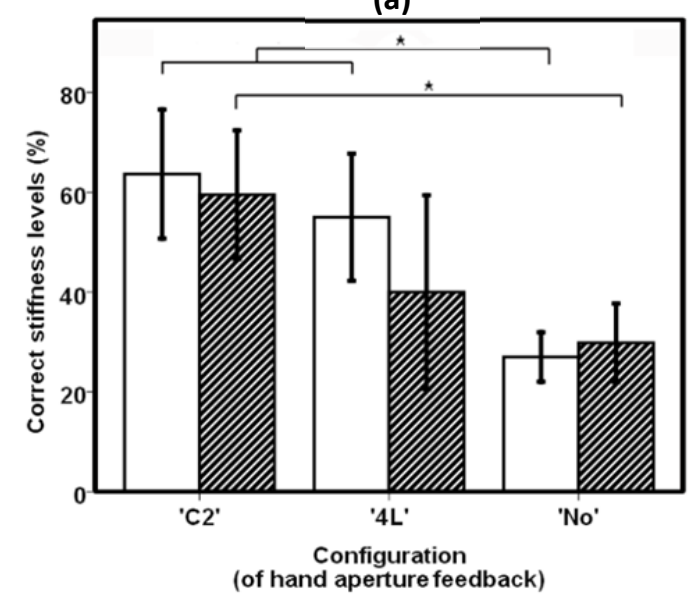

(b)

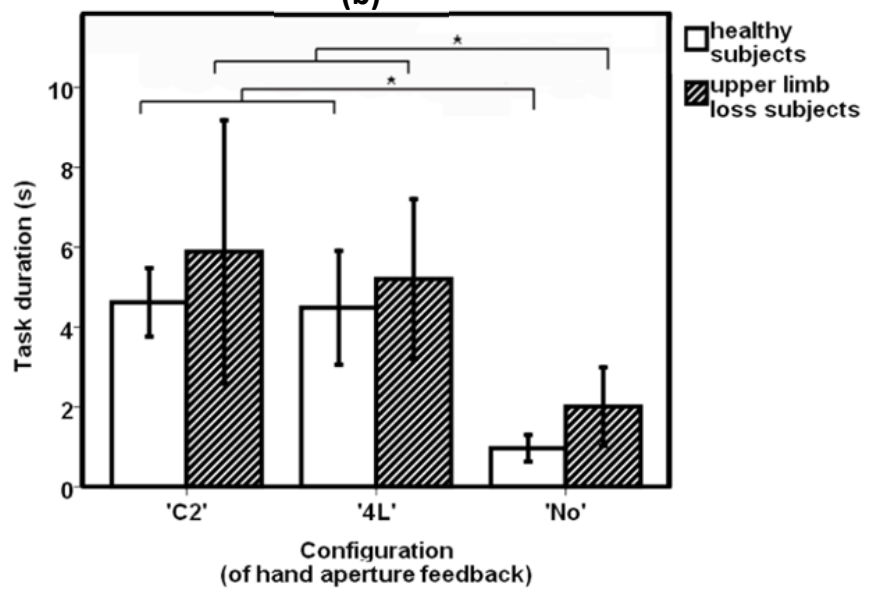

Figure 4: (a) Mean percentages correct stiffness levels and (b) mean task durations with corresponding $\mathbf{9 5 \%}$ confidence intervals. Results are given for 10 healthy subjects and 7 subjects with upper limb loss for three feedback configurations named after the way hand aperture feedback is provided ('C2', '4L', 'No'). * indicates significant differences $(\mathrm{p}<0.05)$.

A general linear model (GLM) was used to perform repeated measures ANOVA to evaluate the differences between the two feedback configurations and to investigate if the percentages stiffness determination where higher compared to the no feedback condition. This analysis showed that stiffness determination via hand aperture feedback through the C2 tactor ('C2') was significantly better compared to the no feedback situation $(\mathrm{p}=0.009)$, but not compared to hand aperture feedback via 4 coin motors $(\mathrm{p}=0.178)$. However, the ' $4 \mathrm{~L}$ ' configuration did not show a significant difference in stiffness determination compared to no feedback $(p=0.257)$. The task duration was significantly increased for both vibrotactile feedback configurations $(\mathrm{p}=0.022$ and 0.008 for the $\mathrm{C} 2$ and $4 \mathrm{~L}$ configuration) compared to no feedback, but no differences in task duration were found between both vibrotactile feedback configurations $(p=0.892)$.

Unpaired samples t-tests were performed to investigate differences in stiffness identification between subjects with upper limb loss and healthy subjects. Tests were performed for both outcome parameters (percentages correctly identified stiffness and task duration) and all three feedback configurations ('C2', '4L' and 'No' feedback). None of the differences between the subjects with upper limb loss and healthy subjects resulted in significance levels below 0.05 .

\section{Discussion}

With vibrotactile feedback about the level of hand aperture alone or in combination with feedback about grasping force, subjects were able to correctly distinguish 4 stiffness levels in $60 \%$ of the cases. In their study on object discrimination through direct nerve stimulation. Horch et al. presented discrimination rates of around $27 \%$ in case of three object sizes and 3 stiffness levels [71]. Discrimination of 2 sizes and 2 stiffness levels resulted in discrimination performances of around 67\%. These values are difficult to compare with our study, because they also included discrimination of object size and the 
stimulation methods are completely different. However, both studies showed the possibilities of feedback to provide stiffness information.

The reported performance percentages with vibrotactile feedback in our study were significantly higher than those achieved without feedback, which shows that vibrotactile feedback indeed provides useful information about the stiffness of an object. However, the percentage of objects which stiffness could be identified correctly out of four options was around $60 \%$, which is rather low. It can be questioned whether this is high enough to be used in daily life object discrimination and if prosthesis users will find it a useful addition to their prosthesis. The moderate subjective scores for usability and intuitiveness of the feedback underline these doubts on the usefulness. Future studies with stiffness feedback in daily life grasping tasks, including longer training periods, should provide more insight on the real benefit of the stiffness feedback.

Although no learning effects in discrimination performance were seen over one experiment, it can be expected that repetition of the experiments will increase the discrimination performance. When the tasks in the experiments are considered motor learning tasks, it can be expected that after a certain period of training, the task duration will decrease, followed by an increase in performance and more subconscious control after a longer training period, according to the steps in motor learning as described by Halsband and Lange [68]. This was already shown by Stepp et al., where after 7 sessions on separate days a significant increase in manipulation performance aided by vibrotactile feedback was seen [138]. They described performance also by the duration of task execution, which decreased over the sessions as well. This decrease in task duration is also seen in our experiments, where a significant decrease in task duration was found between the first and last part of the experiments, which thus can be expected to decrease even further after repetition of the experiments. Learning was induced in our study by showing the subjects the result of their stiffness choice (either too high, too low or correct). This information was used to provide extrinsic feedback in addition to the intrinsic sensory feedback. It can be questioned whether this errorbased, extrinsic feedback was the right performance feedback and it should be investigated in future studies what the best performance feedback should be to reach the most optimal learning. The evaluation of the learning effect was introduced in this study mainly to evaluate differences between configurations. A configuration that requires the least training would be preferred over the others, but no differences in learning between the configurations were found in this study.

\section{Configuration}

All three configurations (hand aperture feedback through 4 coin motors, 8 coin motors, or a single C2 tactor) showed significantly better discrimination performances compared to the no feedback situation, suggesting the usefulness of all three configurations. Percentages of correctly identified stiffness levels in the no feedback situation were around $25 \%$, which is similar to the percentage that will be achieved when guessing the stiffness level out of 4 options. This indicates that no information 
about the stiffness level can be derived from the mouse scrolling or other parts of the setup when no feedback is provided.

Based on the outcome parameters discrimination performance and task duration, no significant differences between the configurations could be found, which was also supported by the similar subjective conclusions (questionnaire results) on the comfort, intuitiveness and usefulness of all three vibrotactile configurations. Our expectation that grasping force feedback via a single $\mathrm{C} 2$ tactor would be most intuitive, because it relates most to the local pressure sensation and hand aperture feedback through an array relates most to a change in position, could however not be confirmed by this study.

The ultimate application of one of these configurations in a myoelectric forearm prosthesis depends on the required number of hand aperture and grasping force levels. The maximum number of levels that can be distinguished, while using amplitude modulation of the $\mathrm{C} 2$ tactor, is limited due to the limited range of stimulation amplitudes. The number of coin motors probably can be larger, but is limited by the space available on the (fore)arm of the user. The inter-stimulator distance of $2 \mathrm{~cm}$ that was used in this study is similar to the 2-point discrimination threshold derived for the forearm for pressure stimuli [142]. In a study by Cholewiak et al. [35] it was stated that with an inter-stimulator distance of $2.5 \mathrm{~cm}$ it is not possible to distinguish perfectly between the stimuli. Therefore, it is not preferable to decrease the inter-stimulator distance. When using a transversal orientation of the array and an inter-stimulator distance of $2 \mathrm{~cm}$, maximally 12 coin motors can be used, based on a mean forearm circumference of $25 \mathrm{~cm}$ for the subjects with upper limb loss.

Hand aperture levels are being used during object reaching as well as during object holding (stiffness determination). To avoid the use of too many hand aperture levels during object reaching, only relatively large objects were used in this experiment. It should be investigated whether stiffness feedback is necessary for small objects and how many hand aperture levels are required to provide this stiffness feedback.

\section{Feedback method}

When grasping force feedback alone was provided, subjects were not able to successfully determine the stiffness of an object, resulting in percentages of correctly identified stiffness levels around the guessing level of 25\%. Hand aperture feedback alone and the combination of hand aperture and grasping force feedback both showed significantly higher discrimination percentages compared to no feedback, while no differences in percentages were found between both feedback methods. These results were highly dependent on the used control method of the virtual hand. During reaching, hand aperture was controlled by mouse scrolling, while during object holding the force was controlled by mouse scrolling in a linear and constant way. This control method was chosen, because it is similar to the control of current myoelectric prostheses, such as the DMC proportional hand prosthesis of Otto Bock, where EMG activity firstly controls the closing of the hand, followed by the control of grasping force when an object is touched. Our finding that hand aperture feedback alone could provide enough information about object stiffness is in contrast to other findings in literature where it is stated that both 
proprioceptive and tactile feedback are important in successful object discrimination $[71,136]$. Our results, could be explained by the fact that during object grasping the grasping force was controlled by the mouse scrolling and therefore could have provided information about the applied force. Furthermore, it is possible that subjects could detect the change in gain between mouse scrolling and the hand aperture feedback (see Figure 2). During grasping, hand aperture is linearly related to the level of mouse scrolling with a fixed gain. However, during the holding phase, the gain of this relation changes due to the stiffness of the grasped object, because from that moment force is controlled by the mouse scrolling. For stiffer objects, more force is needed to achieve the same change in hand aperture and therefore the gain decreases.

If the hand aperture would be controlled by the subjects during object holding, this most likely would have led to the finding that with grasping force feedback alone object discrimination would be possible. This indicates that the chosen control method is also important in object discrimination in addition to the feedback method.

Although no differences were found in percentages correct object discrimination between the combination of hand aperture and grasping force feedback and hand aperture feedback alone, there is a subjective preference for the combination of hand aperture and grasping force feedback to provide the stiffness feedback. Also in the human hand, object discrimination is related to the combination of proprioceptive and tactile feedback [144] and therefore probably more intuitive to use.

An argument against the use of the combination of both feedback methods could be the somewhat larger task duration that is found in comparison to hand aperture feedback alone. It seems to take more time to interpret the combination of both feedback signals, but as was already stated before, the task duration is likely to decrease over time due to the learning effect.

\section{Measurements from subjects with upper limb loss}

Due to amputation of the forearm, sensitivity of the forearm could have been disturbed and thereby the usability of vibrotactile feedback could have been altered. In literature there is no consensus on the effect of amputation on the sensitivity of the stump, ranging from no differences to an increased sensitivity of the forearm stump [66, 73]. Sensitivity in these studies has been characterized by touch thresholds, two-point discrimination thresholds and point localization. Diminished sensations with vibrotactile stimulation are seen in neuropathic diabetes [60], which can also be the cause of (mostly lower limb) amputation and thus result in changed sensitivity in amputees. However, none of the forearm amputations in this study were related to diabetes. In our study, no significant differences in stiffness identification were found between healthy subjects and subjects with upper limb loss, which provides a first indication that vibrotactile stiffness feedback can be useful for subjects with upper limb loss due to traumatic amputation or congenital defects, who would be the ultimate users of the feedback. Although the differences were not significant, the percentages correctly identified stiffness levels were somewhat lower for the subjects with upper limb loss and they also needed more time to perform the tasks. This can probably be explained by the fact that 
the healthy subjects were younger and are usually more experienced computer users. Furthermore, most students did participate in other studies at the university before, while for all subjects with upper limb loss this experience was completely new. Although the characteristics of both subject groups were not matched very well, it did not significantly influence the results and it is expected that the results will be even more comparable between healthy subjects and subjects with upper limb loss if the training time is increased. Two subjects with upper limb loss had to be excluded from this study, because the instructions were too time-consuming due to language problems. However, these subjects with upper limb loss are also possible users of the feedback system and therefore it can be questioned whether this feedback method requires too much instruction and learning time or if it is still acceptable for the users.

Subjects with upper limb loss did not score significantly above guessing level when using the feedback configuration with 4 coin motors for hand aperture feedback and the C2 tactor for grasping force feedback and therefore we assert that this configuration is not useful for subjects with upper limb loss. While using this feedback configuration, subjects with upper limb loss often reported during the experiments that they were not able to feel the change in activation of the coin motors, which was hindered by the C2 tactor. It is likely that the performance would increase over time, but a more intuitive method, requiring less training is necessary for the acceptation of a feedback method in a prosthesis and therefore the other configuration with hand aperture feedback via the $\mathrm{C} 2$ tactor is preferred in this case.

\section{Experimental limitations}

The number of available hand aperture feedback levels (4 or 8) was limited and therefore the stiffness of smaller objects (requiring up to 3 hand aperture levels) was harder to distinguish, because the number of levels in hand aperture feedback to provide the deformation information sometimes was even limited to 1 for these smaller objects. This could have influenced the discrimination performance, but the number of smaller objects was equally presented over all experiments and therefore the effects will be the same over all configurations and feedback methods.

During the experiments it has not been necessary to change the stimulation amplitudes of the vibrotactile stimulators, because they were perceived equally well and no clear adaptation effect occurred. This further emphasizes the preference for vibrotactile feedback over electrotactile feedback, which also requires a session of stimulus adjustments prior to the experiments [157] and seems more sensitive to habituation effects [153].

\section{Conclusion}

Subjects were able to determine virtual object stiffness with the use of combined vibrotactile hand aperture and grasping force feedback or by vibrotactile hand aperture feedback alone. No differences between the feedback configurations were found and the results of subjects with upper limb loss were similar to results from healthy subjects. 
6 - Vibrotactile stiffness feedback

Whether this stiffness feedback is useful for myoelectric prosthesis users in daily life should be further investigated. 


\section{Part 3}

\section{Evaluation of feedback methods}




\section{Chapter 7 - Vibrotactile feedback and EMG control}

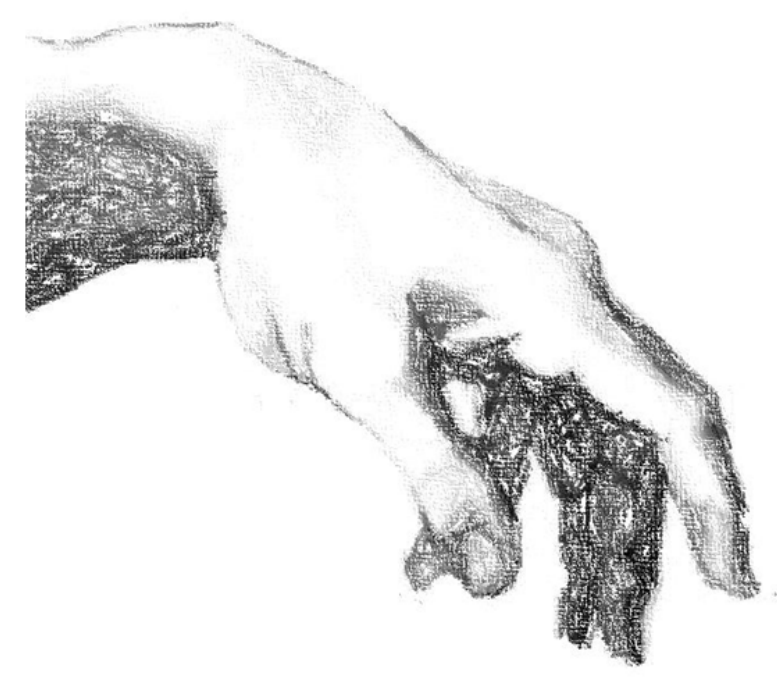

Vibrotactile feedback about hand aperture of a virtual forearm prosthesis controlled by EMG

Authors: Heidi Witteveen, Hans Rietman, Peter Veltink

Submitted 


\section{Abstract}

The number of myoelectric forearm prostheses that is being used on a daily basis remains quite low, among others caused by the lack of sensory feedback. The use of vibrotactile stimulation to provide artificial sensory feedback about grasping force or hand aperture seems a promising approach. In a previous study, vibrotactile hand aperture feedback has been studied in a virtual grasping task, which resulted in an increase in grasping performance. However, the virtual hand was controlled by mouse scrolling, which is not equivalent to the myoelectric (EMG) control of a prosthesis. EMG control is extra attention demanding and therefore possibly influences the interpretability of the feedback and on the other hand the vibrotactile stimulation possibly influences the EMG control signal. In the current study, we have compared the performance in virtual grasping tasks between mouse control and EMG control. It was shown that vibrotactile hand aperture feedback, provided through an array of eight coin motors, increases the grasping performance in the virtual grasping tasks compared to situations without feedback. EMG control did not influence the performance in virtual grasping tasks, but resulted in significantly higher task durations. Furthermore, vibrotactile stimulation did not influence the EMG signal controlling the hand aperture of the prosthesis. A large decrease in task duration over time was seen for the EMG control, indicating a strong learning effect with the EMG control. 


\section{Introduction}

Recent developments in myoelectric forearm prostheses have increased the functionality of the prostheses, but the percentage of myoelectric prostheses that is being used on a daily basis, remains quite low (around 64\% of the patients once fitted with a prosthesis)[18]. To improve the acceptance rate, the addition of artificial sensory feedback has been indicated as one of the major factors [12, 113]. A workshop with representative prosthesis users revealed that grasping force and hand aperture feedback are the most important aspects to be incorporated in myoelectric forearm prostheses [107]. Grasping force feedback is essential in the handling of objects [75], especially because the grasping force that is being applied by the prosthesis cannot be derived visually. The hand aperture of the prosthesis can be derived visually, but this causes a high burden on the visual system and the prosthesis cannot be used subconsciously[143] or in situations where sight on the prosthesis is blocked.

Several attempts have been made to develop artificial non-invasive feedback methods, but none of them have been implemented in commercially available prostheses yet. Examples of grasping force feedback have been described more often than hand aperture feedback. A distinction between early approaches using electrotactile stimulation $[16,115,127]$ and more recent work on vibrotactile stimulation $[28,39,40$, $113,116,123,139,140,159]$ can be made. Vibrotactile stimulation is favorable over electrotactile stimulation, because of the broader and more comfortable range of stimulation amplitudes and their ease of use. In most of these studies either a single C2 tactor (miniature vibrotactile stimulator) or an array of small coin motors is used, mostly showing an increase in grasping performance with the addition of vibrotactile grasping force feedback. The application of EMG (electromyographic) control and grasping force feedback in a real prosthetic hand was evaluated by Chatterjee et al. [28]. An Otto Bock hand was controlled by EMG and the grasping force was measured by a strain gauge. The applied force, 3 discrete levels, was fed back through a frequency modulated C2 tactor signal. Subjects did not show improvements in grasping performance compared to the control situation without feedback.

Hand aperture feedback has only been described in a small number of studies. In an early approach by Prior et al., hand aperture feedback through pulse rate modulation of electrotactile stimulation has been combined with grasping force feedback through amplitude modulation [115]. Their results showed that it was very hard to distinguish between both feedback methods. Recently, feedback about hand grasp configurations, by the use of an array of C2 tactors placed around the waist, has been proposed by Cheng et al. [30]. It seemed feasible to distinguish a large number of hand configurations by rather simple activation patterns of the $\mathrm{C} 2$ tactors, but the time needed to interpret these patterns was high. In another study, hand aperture feedback via an array of eight vibrotactile and electrotactile stimulators on the forearm was evaluated and longitudinal and transversal orientations were compared [157]. A significant improvement in grasping performance in virtual grasping tasks was achieved, while no differences between both orientations were found. A preference for vibrotactile 
stimulation was indicated, because the task duration was significantly lower and electrotactile stimulation was sometimes perceived as less comfortable.

EMG control was only included in three of the above described vibrotactile feedback studies $[28,40,139]$ and the focus of these studies was not on the influence of the EMG control on the feedback and vice versa. However, EMG is the main control input for the myoelectric forearm prostheses and therefore these influences should be evaluated.

In a previous study on vibrotactile hand aperture feedback [157], the hand aperture of a virtual hand was controlled through mouse scrolling. Mouse scroll control was used, because the EMG control performance could be highly variable over subjects and would require a longer training period and therefore undesirably increase the duration of the protocol. In this study, the mouse scroll and EMG control input were compared by evaluation of the performance in virtual grasping tasks, while using vibrotactile hand aperture feedback. The protocol was reduced by selection of the optimal feedback parameters from previous studies.

\section{Methods}

\section{Subjects}

10 healthy subjects $(25.7 \pm 1.8$ yrs. $)$ participated in the experiments. Subjects were included when they did not participate in studies using vibrotactile stimulation before, were between 18 and 65 years, and did not report any sensory or skin problems of the forearm. All subjects were right-handed or used their right hand to control a computer mouse. The mean circumference of the forearm, measured $3 \mathrm{~cm}$ below the elbow, was $26.3 \pm 2.2 \mathrm{~cm}$. All subjects were informed about the experiment via an information letter and all signed informed consent.

\section{Hand aperture feedback}

The feedback method to provide the hand aperture feedback is equivalent to the method used in a previous study [157]. Hand aperture feedback was provided through an array of eight coin motors $(1 \mathrm{~cm}$ diameter, $0.3 \mathrm{~cm}$ height, iNEED (HK) Limited, Shenzhen, China) placed around the forearm, $3 \mathrm{~cm}$ distal to the elbow, with equal inter-stimulator distances. The coin motors were attached to the skin with double-sided tape rings (EEG Kleberinge, GVB-geliMED KG, Bad Segeberg, Germany). Eight discrete hand aperture levels, equally distributed between the fully opened and closed hand, were related to the eight coin motors. Activation of a single coin motor corresponded to one of the eight hand aperture levels. The most dorsal stimulator corresponded to the fully opened hand and when closing the hand, the activation moved counterclockwise around the arm. The amplitude of stimulation of each stimulator was kept constant at $2.5 \mathrm{~V}$, which resulted in clearly tangible sensations. 


\section{Experimental setup}

A virtual setup was used to exclude information about the hand aperture coming from the healthy sensory pathways. A virtual hand, which hand aperture was controlled by the subjects, was simulated on a computer screen. Together with the hand, circular objects with varying sizes were simulated. Eight different object sizes were used, corresponding to eight discrete levels of hand aperture that are required to hold the object. For each experimental session, 40 objects, with randomly selected sizes, were presented. The task for the subjects was to realize a hand aperture that just fitted the presented object and to hold that hand position for three seconds after which the next object was shown.

\section{Control methods}

Two methods to control the hand aperture of the virtual hand were compared: control via mouse scrolling, as used in our previous study[157], and control via surface EMG, as used in myoelectric prostheses. For mouse scroll control, each mouse scroll was related to a change in hand aperture. The 'clicks' from the scroll wheel were removed and a random gain, relating the level of scrolling to a change in hand aperture, was applied. This was done to rule out all possible information about the hand aperture coming from the mouse scrolling and force the subjects to fully rely on the vibrotactile feedback.

For EMG control of hand aperture, surface EMG from the wrist extensor and wrist flexor muscles was measured. The optimal location for the EMG electrodes was found by palpation of the muscle bellies during contraction of wrist extensors and flexors. A bipolar derivation, using two electrodes per muscle, was used and a reference electrode was placed at the dorsal side of the wrist. A custom-build EMG amplifier with a sampling rate of $100 \mathrm{~Hz}$ and a Bluetooth connection with the measurement computer was used to create the control signal. An envelope of the EMG signals was created by applying a second-order Butterworth high-pass filter with a cut-off frequency of $10 \mathrm{~Hz}$, followed by the rectification of the signal and the application of a second-order Butterworth lowpass filter with a cut-off frequency of $0.5 \mathrm{~Hz}$.

The envelope control signal was calibrated against the maximal flexion and extension force separately. To measure this force, an ATI force sensor (Mini45, ATI Industrial Automation, Apex, NC, USA) was incorporated in the setup between the hand support and the forearm support (see Figure 1). The torque, exerted during wrist flexion and extension, was measured and displayed in a graph on the computer screen. The subjects were asked to perform a maximal voluntary contraction (MVC). Afterwards, a red line at $60 \%$ of the measured MVC was shown in the torque graph and the subjects were asked to perform a muscle contraction that kept the measured torque around the red line. At that moment, the corresponding value of the envelope EMG signal was set to -1 for the wrist extensor and +1 for the wrist flexor, corresponding to maximal hand opening and closing respectively. A single control signal was created by adding up both signals and the range between -1 and 1 was divided into discrete hand aperture levels with equal intervals. During muscle relaxation, the hand aperture was kept constant at the current 
position. Torques were only measured during the calibration procedure and not during the actual measurements.

EMG was measured under isometric conditions to approach the muscles contractions for control of a myoelectric forearm prosthesis and to make it easier to activate the wrist extensor and flexor muscles separately from each other. To achieve this, a setup, fixed to the table, supporting the hand and the forearm was used (see Figure 1).

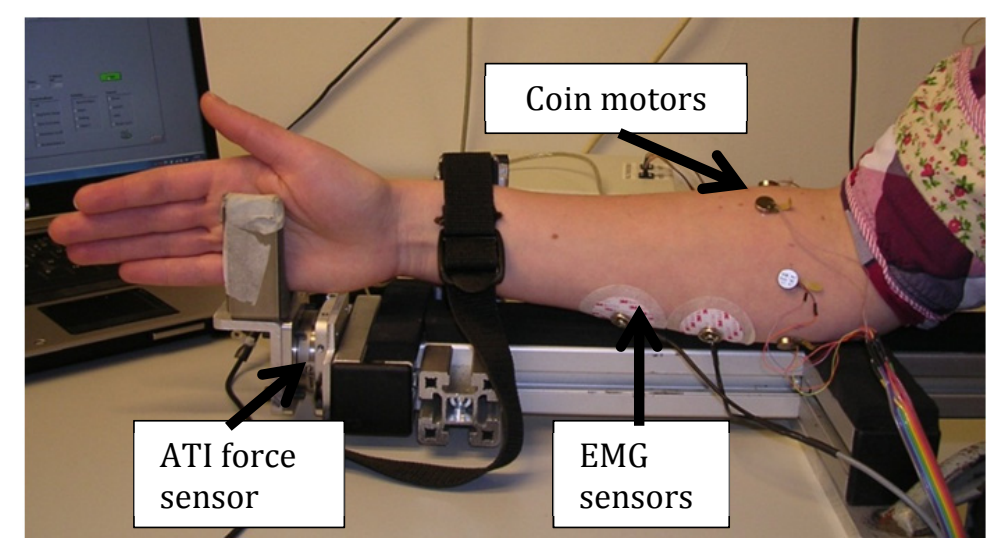

Figure 1: Measurement setup fixating the forearm of the subjects. Between the hand and the arm support a force sensor (only used for the calibration) was placed. EMG electrodes were placed at the muscle bellies of the wrist flexors and extensors. 8 coin motors were placed around the forearm

\section{Protocol}

The performance in grasping tasks is compared for three feedback conditions. During the first condition visual feedback and vibrotactile feedback were provided simultaneously. Visual feedback was provided by the visualization of the moving virtual hand on the computer screen and the virtual object to be grasped. This visual feedback condition also served as a training period, because vibrotactile feedback was provided simultaneously. Subjects were asked to give attention to the vibrotactile feedback and try to remember the relation between the hand aperture and the feedback. During the second condition, only vibrotactile feedback about the hand aperture was provided and the visual feedback was blocked (no hand visible). The object to be grasped was only shown for the first 0.5 seconds. During the third condition no visual and no vibrotactile feedback was provided. This last condition was the control condition for the experiments and is representative for situations where sight on the prosthesis is blocked. All three conditions were performed for mouse scroll control as well as for EMG control. A summary of the experimental conditions is shown in Table 1. The order of the control inputs was randomized over the experiments to exclude learning effects, but the visual feedback condition was always performed before the vibrotactile feedback condition, because the visual feedback condition was also used as training condition. 
Table 1: Overview of the feedback conditions and control inputs as used during the experiments.

\begin{tabular}{|c|c|c|c|}
\hline $\begin{array}{c}\text { Task } \\
\text { number }\end{array}$ & Feedback condition & Control input & \# objects \\
\hline 1 & Visual + vibrotactile feedback & Mouse scroll & 40 \\
\hline 2 & Vibrotactile feedback & Mouse scroll & 40 \\
\hline 3 & No feedback & Mouse scroll & 40 \\
\hline 4 & Visual + vibrotactile feedback & EMG & 40 \\
\hline 5 & Vibrotactile feedback & EMG & 40 \\
\hline 6 & No feedback & EMG & 40 \\
\hline
\end{tabular}

\section{Data analysis}

The level of hand aperture as applied by the subjects was compared to the required hand aperture to exactly grasp the shown object. Based on this comparison the percentages correct hand apertures and the percentages hand apertures deviating up to one level from the required hand aperture were calculated. This latter parameter was used to provide another, less strict, accuracy measure. Furthermore, the time needed by the subjects to complete the tasks was determined. Finally, subjects were asked to indicate the perceived comfort of the stimulation and the usability of the feedback on a VAS scale (ranging from not comfortable or not useful at all to very comfortable or very useful) for each feedback condition and both control inputs.

Each outcome parameter was evaluated for all three feedback conditions and both control inputs. Differences were statistically analyzed, using a repeated measures ANOVA test with a significance level of 0.05 . When necessary, Bonferroni corrected paired samples t-tests were performed to evaluate differences between feedback conditions.

\section{Results}

The hand aperture EMG control signal was based on EMG from the wrist extensor and wrist flexor. An example of a period of subsequent activation of both muscles and the resulting control signal is shown in Figure 2. Activation of the wrist extensor resulted in opening of the hand and activation of the wrist flexor resulted in closing of the virtual hand. The average delay between muscle activity onset and the change in control signal was determined to be around $40 \mathrm{msec}$. Each discrete level of hand aperture was related to activation of one of the eight coin motors, which is also shown in Figure 2. 


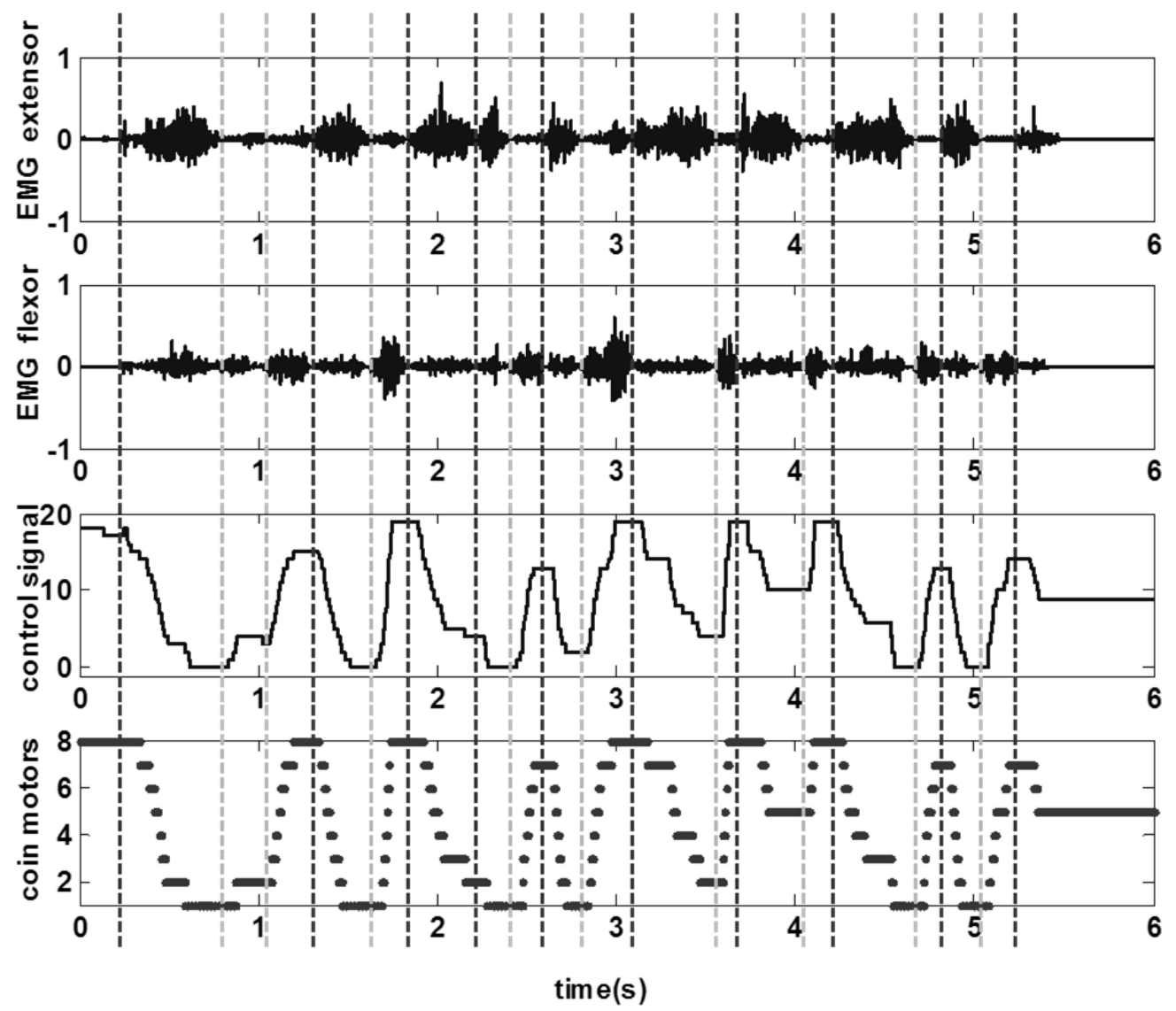

Figure 2: Normalized EMG control signals measured from the wrist extensor (first row) and the wrist flexor (second row) after high-pass filtering with a cut-off frequency of $2 \mathrm{~Hz}$. The resulting control signal (third row) and the corresponding activated coin motors (fourth row). The vertical lines indicate the onset of the extensor (dashed black line) and flexor (dashed grey line) muscle activity respectively

Percentages correctly applied hand apertures and percentages hand apertures deviating up to one level were calculated for each feedback condition and both control inputs. The results, averaged over the 10 subjects, are presented together with the corresponding standard deviations in Figure $3 a$ and $b$.

Repeated measures ANOVA revealed that there were no differences in percentages correct hand apertures between tasks controlled via mouse scrolling and via EMG $(\mathrm{p}<0.228)$, but percentages hand apertures deviating up to one level were just significantly lower $(\mathrm{p}=0.022)$ for EMG control. However, Bonferroni corrected t-tests per feedback condition, did not reveal any significant differences. There was a significant effect of the feedback condition on the percentages correct hand apertures as well as the percentages hand apertures deviating up to one level ( $p$-values $<0.001$ ) and therefore post-hoc t-tests were performed to further evaluate these differences. It was shown that the percentages correct hand apertures and hand apertures deviating up to one level differed significantly between all three feedback conditions ( $p$-values between $<0.001$ and 0.002), thus higher percentages for additional visual feedback compared to vibrotactile feedback only and higher percentages for vibrotactile feedback only compared to no feedback. 
(a)

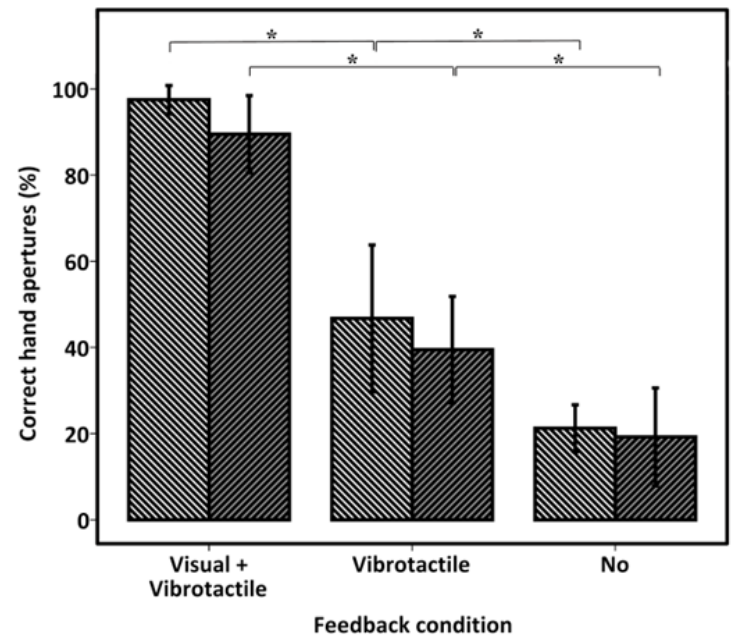

(b)

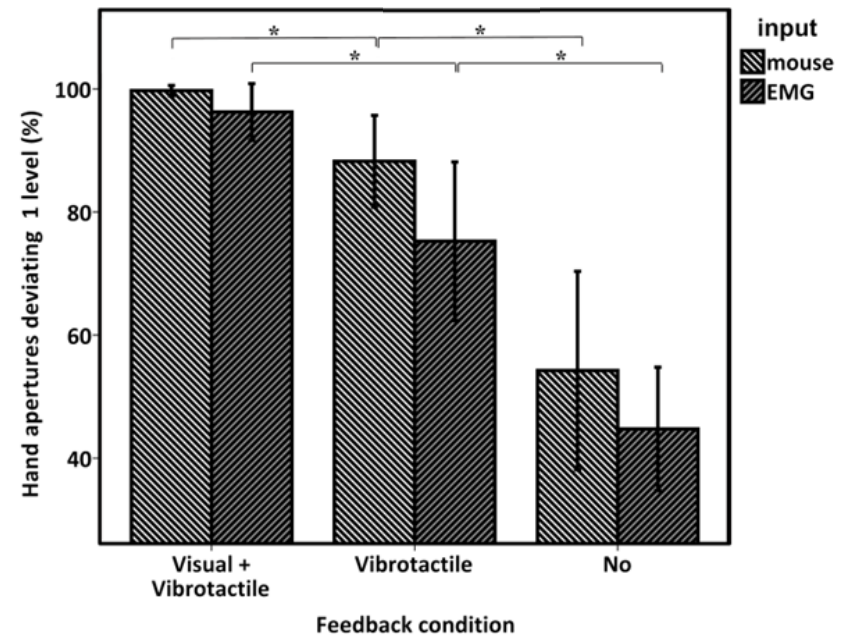

Figure 3: (a) Mean percentages and corresponding standard deviations of correct hand apertures and (b) mean percentages hand apertures deviating up to 1 level and corresponding standard deviations for each feedback condition and both control inputs. Significant differences $(p<0.05)$ are indicated with asterisks

Task durations were calculated as the time needed to complete the whole grasping task. In Figure 4 the mean task durations are presented for all three feedback conditions and both control inputs. The feedback conditions were all applied in the same order, starting with visual and vibrotactile feedback simultaneously and ending with no feedback. A clear decrease in task duration over time was seen for the EMG input, while for the mouse scroll input the task durations were stable. Repeated measures ANOVA showed that both the feedback condition and the control input significantly influenced the task duration, but also a significant interaction effect between both factors was found. Therefore, post-hoc Bonferroni corrected t-tests, were performed for both factors separately. For all three feedback conditions a significant increase in task duration was found for the EMG controlled tasks compared to mouse scroll controlled ( $p$-values between 0.011 and 0.025 ). Only for the EMG controlled tasks, significant differences between feedback conditions were found. Task durations were larger for the visual feedback and vibrotactile feedback only conditions compared to the tasks without feedback ( $p=0.011$ and $p=0.003$ respectively), while differences were not significant between the visual feedback and the vibrotactile feedback only conditions. 


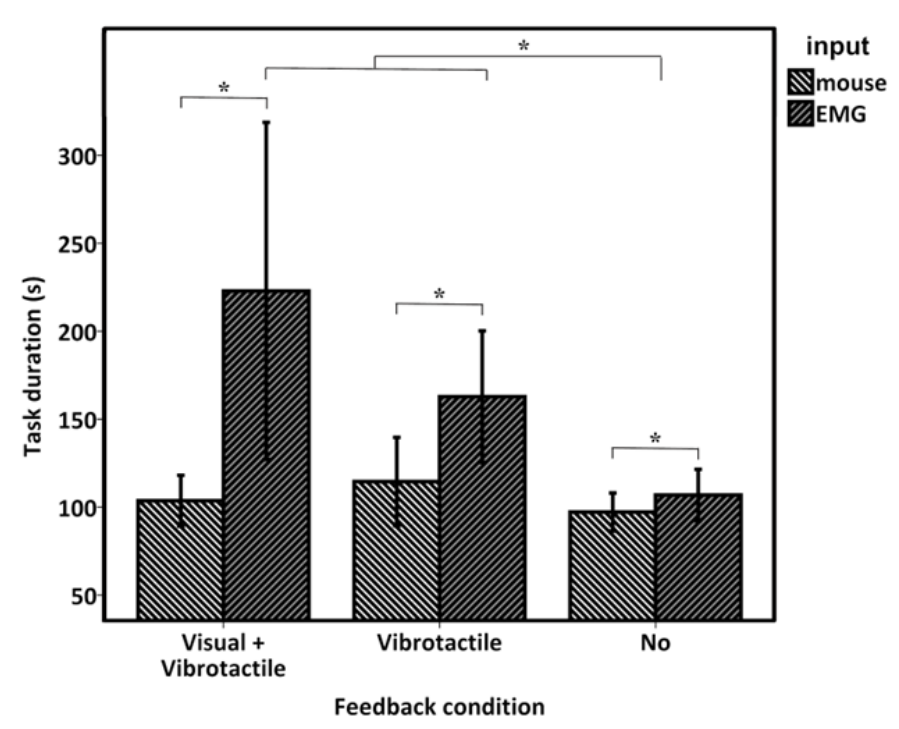

Figure 4: Averaged task durations and corresponding standard deviations for each feedback condition and both control inputs. Significant differences $(p<0.05)$ are indicated with asterisks.

\section{Subjective results}

The summarized results of the subjective interpretation of the comfort and usability of the vibrotactile hand aperture feedback are presented in Figure 5. It was shown, by repeated measures ANOVA, that the comfort of the stimulation was significantly influenced $(\mathrm{p}=0.04)$ by the used control input, which was lower for EMG control.

Repeated measures ANOVA also showed that the usability of the hand aperture feedback was significantly different between the feedback conditions. Visual feedback and vibrotactile feedback were both perceived as being more useful than the no feedback condition ( $p$-values $\leq 0.001$ ), but no differences were found between the visual feedback and the vibrotactile feedback only conditions.

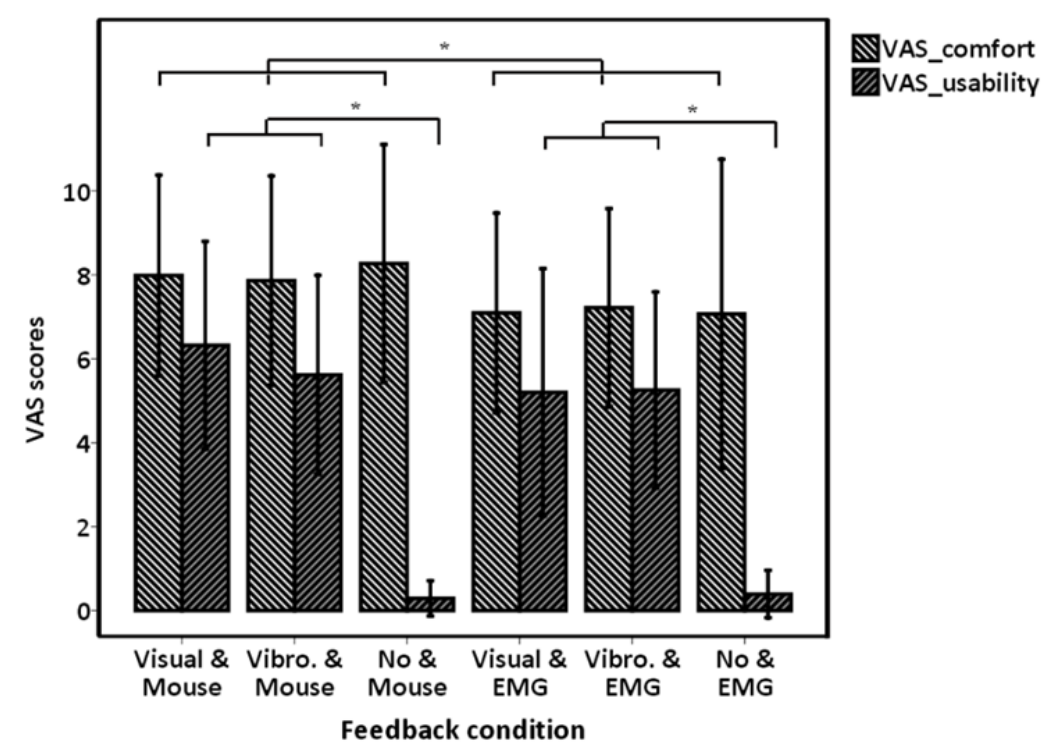

Figure 5: Mean and standard deviations of the VAS scores for comfort and usability during the hand aperture feedback experiments. Scores are given for each feedback condition and both control inputs 


\section{Discussion}

\section{EMG control}

Subjects were able to control the hand aperture of the virtual hand by EMG from the wrist flexors and extensors, which was reflected in a clear control signal. Furthermore, the simultaneously applied vibrotactile stimulation did not influence the EMG signals and the resulting control signal, because during coin motor stimulation the control signal did not change and subjects were still able to control the hand aperture successfully. The distance between the coin motors and the EMG electrodes ranged, depending on the location of the active coin motor, on average between $2 \mathrm{~cm}$ and $13 \mathrm{~cm}$. In a study by Chatterjee et al. [28] the minimal distance between a C2 tactor (larger and linear vibrotactile stimulator) and EMG control electrodes was empirically determined to be $5 \mathrm{~cm}$. However, this $\mathrm{C} 2$ tactor is larger and more forceful in comparison to the coin motors, resulting in higher vibration amplitudes, which possibly influences the EMG signal more due to larger skin movements. Based on our results, we conclude that the distance between the coin motors and the EMG electrodes can be less than $5 \mathrm{~cm}$ or even less than $2 \mathrm{~cm}$.

With mouse scroll control, the task durations were equal for all three feedback conditions, showing that the vibrotactile stimulation did not influence the task duration. When comparing the task durations with hand aperture control via mouse scroll and via EMG it was seen that EMG control requires significantly more time to complete the tasks. It seems more difficult to perform the virtual grasping tasks with EMG, which was also reflected in the somewhat lower performances (percentages correct hand apertures) with EMG control compared to mouse scroll control in the visual feedback condition. Chatterjee et al. already showed that even with visual feedback, tasks cannot be performed in a perfect way with EMG control [28]. A trend of decreasing task durations was seen over the course of the experiments, with the highest task durations for the visual feedback condition, which was always performed first, indicating a strong learning effect with EMG control. As this learning effect was already seen after training with 40 objects (visual feedback condition), it can be expected that the task duration will decrease even further after more sessions of training with EMG control. A decrease induced by training, expressed in higher movement velocities, was also shown in a study by Stepp et al. on vibrotactile force feedback [139], who also showed an increase in performance over time.

Intuitively, EMG control would provide more information about the hand aperture of the prosthesis compared to mouse scroll control, because the amplitude of muscle contraction is directly related to the change in hand aperture. However, when no vibrotactile and no visual feedback is provided and the subjects had to fully rely on the EMG or mouse scroll control, no differences in performance were found between both control inputs. Furthermore, the found percentages were all around guessing level, showing that no information about the hand aperture could be derived from the control input. 


\section{Hand aperture feedback}

With vibrotactile hand aperture feedback only, subjects were able to reach the required hand aperture in around $40 \%$ of the cases and when looking at the deviations up to one level of hand aperture, the percentages increased to around $80 \%$. Both percentages were significantly higher (for both control methods) compared to the no feedback condition, which indicates the usefulness of the vibrotactile hand aperture feedback. This holds especially when sight on the prosthesis is blocked, because with visual feedback the performance is still significantly higher. The results of this study are highly similar to the results of our previous study on vibrotactile hand aperture feedback [157], where the virtual hand was only controlled by mouse scroll.

For both control inputs the performance results were significantly higher compared to the no feedback condition. From an earlier study [156] on hand aperture feedback and external distraction it was already concluded that vibrotactile hand aperture feedback is not influenced by intermediate levels of distraction, which seems also to be the case for EMG control.

For mouse scroll control, task durations were not influenced by the feedback conditions (similar task durations with the visual feedback, vibrotactile feedback only and without feedback). This indicates that vibrotactile feedback did not require extra time to interpret the feedback and indicates that the found learning effects were fully caused by the use of EMG control and not by the vibrotactile feedback.

The usefulness of vibrotactile hand aperture feedback was also indicated by the responses of the subjects, who clearly perceived the vibrotactile feedback as more useful compared to the no feedback conditions. Vibrotactile feedback only was indicated even as useful as visual feedback combined with vibrotactile feedback, although the performance was significantly lower for the vibrotactile feedback only condition. Visual feedback was probably not perceived as extremely useful, because this condition was always performed first and in combination with the vibrotactile feedback and therefore required more attention from the subjects to learn to use the feedback. The vibrotactile feedback was indicated as being very comfortable, which encourages the use of vibrotactile feedback methods in the future. With EMG control the comfort was indicated lower compared to the mouse scroll input, which is probably caused by the setup that was used to fixate the forearm during EMG control.

\section{Methodological considerations}

EMG control was introduced in this study to approach the situation of myoelectric forearm prostheses, but still a virtual setup was used. This virtual setup enabled the inclusion of healthy subjects, because in this way their intact sensory pathways could be blocked. In a previous study it was already shown that there were no differences in grasping performance between healthy subjects and amputees or congenital defect subjects in a virtual grasping task with hand aperture feedback [157]. Nevertheless, it should be investigated whether the results found in this study are also applicable for vibrotactile feedback implemented in commercial myoelectric forearm prostheses. 
An array of coin motors, placed transversally around the forearm, was used to provide the hand aperture feedback, which was already shown to be successful in an earlier study on vibrotactile hand aperture feedback [157]. The transversal orientation was chosen instead of a longitudinal orientation, because this array would fit completely within a prosthesis cover. However, the optimal stimulation array configuration should be investigated, focusing on the application in a real prosthesis. Furthermore, the addition of vibrotactile grasping force feedback, possibly through amplitude modulation of a single C2 tactor [141, 159], is recommended and its influence on the hand aperture feedback should be investigated in future studies.

\section{Conclusion}

This study has shown that vibrotactile hand aperture feedback is successful in virtual grasping tasks and is a next step towards the implementation of feedback in myoelectric forearm prostheses, because it is shown that (1) vibrotactile hand aperture feedback increases the grasping performance in situations where no visual feedback is available, (2) the interpretation of vibrotactile feedback is not influenced by the EMG control and (3) the vibrotactile feedback did not affect the EMG control signal. 
7 - Vibrotactile feedback and EMG control

$\frac{\text { ? }}{\frac{0}{0}}$ 
Chapter 8 - Vibrotactile feedback for amputee and congenital defect patients

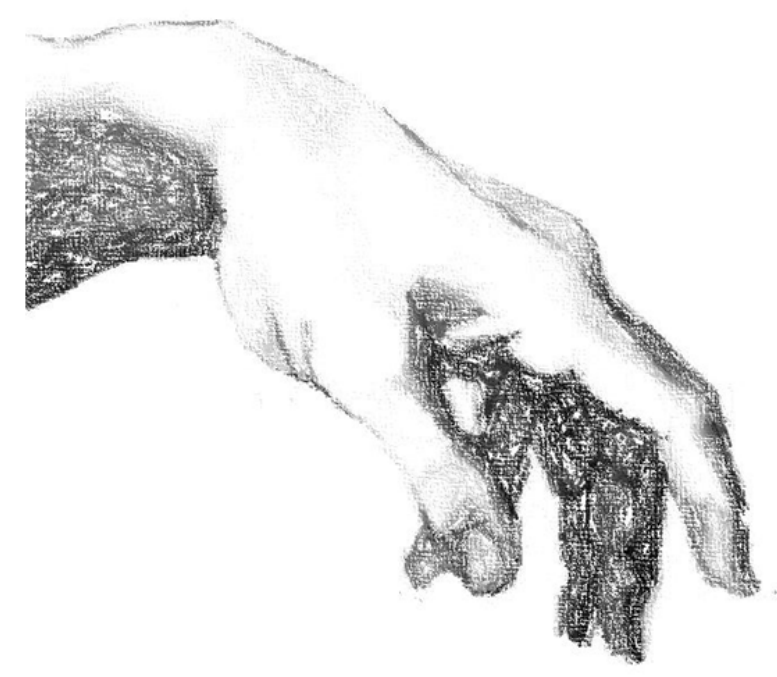

Vibrotactile grasping force and hand aperture feedback for myoelectric forearm prosthesis users

Authors: Heidi Witteveen, Hans Rietman, Peter Veltink

Accepted for publication in: Prosthetics and Orthotics International, 2014 


\section{Abstract}

User feedback about grasping force and hand aperture is very important in object handling with myoelectric forearm prostheses, but is lacking in current prostheses. Vibrotactile feedback increases the performance of healthy subjects in virtual grasping tasks, but no extensive validation on potential users have been performed. The goal of this study was to investigate the performance of upper limb loss subjects in grasping tasks with vibrotactile stimulation, providing hand aperture and grasping force feedback.

10 subjects with upper limb loss performed virtual grasping tasks while perceiving vibrotactile feedback. Hand aperture feedback was provided through an array of coin motors and grasping force feedback through a single miniature stimulator or an array of coin motors. Objects with varying sizes and weights had to be grasped by a virtual hand. Percentages correctly applied hand apertures and correct grasping force levels were all higher for the vibrotactile feedback condition compared to the non-feedback condition. With visual feedback, the results were always better compared to the vibrotactile feedback condition. Task durations were comparable for all feedback conditions.

Vibrotactile grasping force and hand aperture feedback improves grasping performance of subjects with upper limb loss. However, it should be investigated whether this is also of additional value in daily life tasks. 


\section{Introduction}

Myoelectric forearm prostheses are being developed to compensate for the missing of a hand due to amputation or a congenital defect. However, only $64 \%$ of the fitted myoelectric prostheses is being used by their owner on a daily basis [18], which is among others caused by the limited functionality of the prosthesis and the lack of sensory feedback $[12,18]$. From a workshop with representative myoelectric forearm prosthesis users, it is concluded that information about grasping force and hand aperture is the most important to be fed back to a prosthesis user [107]. Popular methods to provide the artificial feedback non-invasively are through electrotactile, mechanotactile or vibrotactile stimulation of the skin [10,78]. Although mechanotactile stimulation is most promising because of the modality-matching that results in the best feedback performances $[10,106]$, recent emphasis is mostly on vibrotactile stimulation due to developments in miniaturization of the stimulators [76]. The focus of most studies is on vibrotactile user feedback about grasping force, provided through a single C2 tactor (miniature vibrotactile stimulator) [28, 40, 140, 141], a single coin motor [113, 116] or an array of coin motors [123]. In another study the use of a single C2 tactor and an array of coin motors was compared [159].

Although feedback about hand aperture is also indicated to be very important [107], only a few studies evaluating hand aperture feedback have been found. Electrotactile stimulation to provide hand aperture feedback has been described, both non-invasively stimulating the skin [115] as well as invasively by direct nerve stimulation [50]. In a recent approach, an array of $\mathrm{C} 2$ tactors on the waist was used to provide vibrotactile feedback about several hand configurations [30]. A comparison between electrotactile and vibrotactile stimulation to provide hand aperture feedback, using an array of stimulators, has been performed as well [157]. Another approach is the use of skin stretch feedback to provide proprioceptive information, which in some cases was superior over vibrotactile feedback $[15,155]$.

In summary, most studies on grasping force and hand aperture feedback showed an increase in grasping performance, but there is no consensus on the optimal stimulation methods. Only in around half of the aforementioned studies the feedback methods were evaluated on amputees and the number of subjects in these studies was relatively low (1-6 amputees). In all these studies, no large differences between healthy subjects and subjects with upper limb loss were found, but this evaluation was not the main focus of these studies.

Several studies have compared the sensitivity of the residual limb of amputees and the healthy limb, but there is no consensus whether sensory thresholds are similar [73]. Older studies reported a better spatial acuity for amputees, lower touch and two-point discrimination thresholds and lower errors in point localization, explained by the reorganization of central sensory mappings after amputation [66, 146]. However, more recent studies on healthy subjects showed that differences in spatial acuity are more likely caused by differences in attention given to the stimulation site. After amputation the attentional resources are spread over a smaller body area, probably resulting in a better spatial acuity $[97,101]$. Other studies found no differences in tactile detection 
thresholds, two-point discrimination, force discrimination and vibration thresholds between the amputated and sound limb [24,73] or between the sound limb and the reinnervated skin after targeted reinnervation [126, 129]. Higher tactile thresholds and two-point discrimination thresholds were only found at the scar tissue [73]. Furthermore, the sensitivity did not change over time, except for some stabilization towards normal values at the scar tissue [74]. To our knowledge, no studies have been performed on sensitivity abnormalities in subjects with congenital transradial reduction defects.

Based on the reviewed literature it cannot be predicted how amputees or subjects with congenital upper limb defects would perform in vibrotactile feedback experiments compared to healthy subjects. Therefore, in this study the performance of subjects with a transradial amputation or congenital reduction defect in virtual grasping tasks is evaluated, while vibrotactile grasping force and hand aperture feedback is provided.

\section{Methods}

\section{Subjects}

Subject characteristics are summarized in Table 1. Subjects with upper limb loss were recruited by rehabilitation physicians of the local rehabilitation center. Subjects were included when they had no experience with vibrotactile stimulation, no severe skin or sensory problems of the residual limb, were able to understand the tasks and able to control the virtual setup with their sound arm. Every subject was informed about the study via an information letter and all signed informed consent. The study protocol is approved by the local medical ethical committee (Medisch Etische ToetsingsCommissie Twente).

Table 1: Summary of the subject characteristics. Mean values and standard deviations are given. amput. = amputation, cong. $=$ congenital defect, $\mathrm{C}=$ cosmetic, $\mathrm{M}=$ myoelectric, $\mathrm{B}=$ body-powered prosthesis

\begin{tabular}{|l|l|l|l|l|l|l|l|}
\hline$\#$ & age (yrs.) & $\begin{array}{l}\text { stump } \\
\text { length (cm) }\end{array}$ & $\begin{array}{l}\text { stump } \\
\text { circum. (cm) }\end{array}$ & $\begin{array}{l}\text { male / } \\
\text { female }\end{array}$ & $\begin{array}{l}\text { amput. } \\
\text { / cong. }\end{array}$ & $\begin{array}{l}\text { yrs. after } \\
\text { amput. }\end{array}$ & $\begin{array}{l}\text { prosthesis } \\
\text { (C/M/B/No) }\end{array}$ \\
\hline 10 & $52.7 \pm 9.3$ & $13.1 \pm 5.2$ & $24.6 \pm 2.2$ & $8 / 2$ & $6 / 4$ & $18 \pm 18$ & $6 / 2 / 1 / 1$ \\
\hline
\end{tabular}

\section{Materials}

Two vibrotactile stimulation methods were used to provide feedback: amplitude and position modulation. A C2 tactor (Engineering Acoustics Inc., Casselberry, FL, USA) was used to provide the amplitude modulated vibrotactile stimulation. The $\mathrm{C} 2$ tactor $(3 \mathrm{~cm}$ diameter and $0.5 \mathrm{~cm}$ height) is activated at $250 \mathrm{~Hz}$, corresponding to the resonance frequency of the tactor and the frequency at which the main mechanoreceptors in the forearm skin, the Pacinian corpuscles, are most sensitive. An array of eight coin motors ( $1 \mathrm{~cm}$ diameter and $0.3 \mathrm{~cm}$ height) was used to provide the position modulated vibrotactile stimulation. The activation voltage of the coin motors (iNEED (HK) Limited, Shenzhen, China) was kept constant at $2.5 \mathrm{~V}$ which corresponds to stimulation frequencies between 50 and $100 \mathrm{~Hz}$, depending on the skin characteristics. The coin 
motors and $\mathrm{C} 2$ tactor were both attached to the skin by double-sided tape rings (EEG Kleberinge, GVB-geliMED KG, Bad Segeberg, Germany).

\section{Grasping force feedback}

Grasping force feedback was provided either through an array of eight coin motors placed around the forearm or through a single $\mathrm{C} 2$ tactor placed on the residual limb. The coin motors were placed $3 \mathrm{~cm}$ distal to the elbow and equally distributed around the arm, resulting in inter-stimulator distances of $3.1 \pm 0.3 \mathrm{~cm}$. Each of eight discrete grasping force levels, as applied by the subjects, was related to activation of one of the eight coin motors. Activation of the most dorsal coin motor corresponded to the lowest grasping force level and with increasing force the activation was moved counterclockwise around the arm. The $\mathrm{C} 2$ tactor was placed at the dorsal side of the forearm, halfway the elbow and the tip of the residual limb. Eight discrete grasping force levels were related to eight amplitude levels, ranging from 1 to $4.5 \mathrm{~V}$ with constant intervals of $0.5 \mathrm{~V}$, whereby $1 \mathrm{~V}$ corresponded to the lowest force level.

\section{Hand aperture feedback}

Hand aperture feedback was provided through an array of eight coin motors. Each of eight discrete hand aperture levels, ranging from a fully opened to a closed hand, were related to activation of a single coin motor. Two orientations of the array, transversal and longitudinal, were investigated. For the transversal orientation, the coin motors were placed around the forearm, similar as for the grasping force feedback. The most dorsal stimulator corresponded to the fully opened hand and for each decrease in hand aperture, the activation of the stimulators moved counterclockwise around the arm. For the longitudinal orientation, the coin motors were placed at the dorsal side of the forearm. The same inter-stimulator distance as used for the transversal orientation was used, which was achieved by placing stimulators on the upper arm. Activation of the most distal coin motor was related to the closed hand and the most proximal coin motor to the fully open hand.

\section{Virtual setup}

A virtual setup was created in Labview (Labview 2010 SP1, National Instruments, Austin, TX, USA), showing an opening and closing hand for the hand aperture feedback experiments (see Figure 1a) or a hand holding objects for the grasping force experiments (see Figure 1b). The hand aperture and the grasping force were controlled by the subjects through mouse scrolling, whereby forward scrolling was linearly related to closing of the hand or an increase in grasping force. The gain between the level of mouse scrolling and the resulting change in hand aperture or grasping force was randomized per object. Furthermore, the 'clicks' that are normally felt when scrolling were removed to exclude every information linking the mouse scrolling to the hand aperture or grasping force, forcing subjects to fully rely on the vibrotactile feedback. For the hand aperture feedback experiments, a virtual circular object was displayed between the thumb and first finger, which had to be grasped by the subjects. Eight 
different object sizes were simulated and presented in random order, up to a total of 40 objects per task. Each discrete level of hand aperture was related to one of the eight object sizes and activation of one of the coin motors. For the grasping force feedback experiments, eight different object weights were simulated, corresponding to eight discrete grasping force levels necessary to successfully hold the object. The weight of the object was visually represented by the color of the object.

(a)

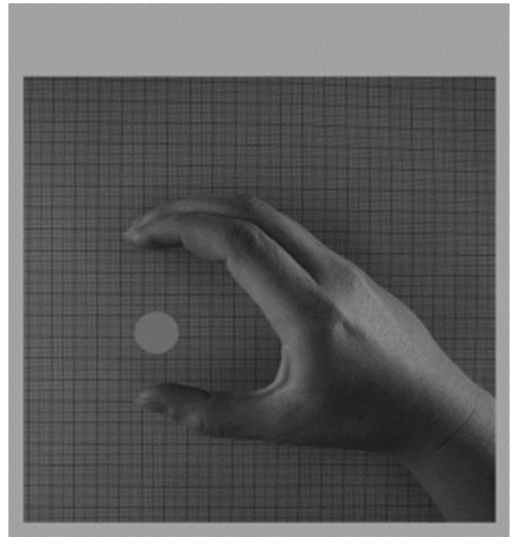

(b)

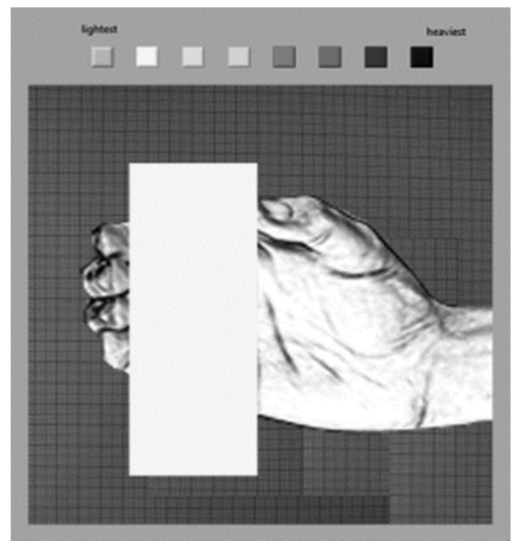

Figure 1: Virtual representation of a grasping hand and objects with varying sizes and weights, used for (a) hand aperture feedback experiments and (b) grasping force experiments

\section{Protocol}

Subjects were seated behind a laptop showing the virtual setup. Subjects controlled the mouse with their sound hand, while feedback was provided to the residual limb.

The grasping force feedback experiments consisted of a training (20 objects) and an experimental session ( 40 objects), both with feedback via the $\mathrm{C} 2$ tactor and the coin motor array. Subjects were asked to apply the required grasping force by mouse scrolling. After 2 seconds the result was shown, which was a squeezed object when the force was too high or a dropped object when the force was too low. If the applied grasping force was correct, the next object was shown, otherwise the same object was shown again with a maximum of 5 trials. During the experimental session, the result of the applied grasping force was not shown and subjects were asked to apply the right grasping force as fast as possible and after 3 seconds the next object was shown.

For the hand aperture feedback experiments, two sessions for both array orientations were performed: during the first session the virtual hand and the object were shown during the whole task, while for the second session the hand was not shown at all and the object was only shown for 0.5 seconds. The first session is called the visual feedback session (in combination with vibrotactile feedback) and is used to learn how to relate the coin motor activation to the level of hand aperture. During the second session, the subjects had to rely on the vibrotactile feedback, simulating situations where visual feedback is not available.

The order of the array orientations and the feedback configurations was randomized to compensate for possible learning effects. A control measurement without vibrotactile and visual feedback was performed after each feedback experiment. 


\section{Statistical analysis}

Based on the required hand aperture and grasping force, percentages correct hand apertures and grasping forces were calculated. Furthermore, percentages hand apertures and grasping forces deviating up to one level were calculated. This latter parameter was used to provide another measure of accuracy, which does not depend on the exact localization or amplitude perception of the stimulus. The last parameter used in the analysis was the task duration, which was calculated as the time taken by the subjects to reach the final hand aperture or grasping force level summed over all objects. Repeated measures ANOVA tests, with a significance level of 0.05, were used to evaluate differences between the feedback configurations. The configurations were included as factor in the analysis with three and five levels for the grasping force and hand aperture feedback experiments respectively.

\section{Comparison with healthy subjects}

Results are compared with the outcomes from two separate studies on healthy subjects on vibrotactile hand aperture [157] and grasping force feedback [159]. In comparison to the protocol of this study, there were two deviations: (1) For healthy subjects, the coin motors were equally distributed between the elbow and wrist and not placed on the upper arm, and (2) healthy subjects controlled the mouse by their dominant (computer) hand, while feedback was given at the same arm. Results between subjects with upper limb loss and healthy subjects are compared via unpaired samples t-tests.

\section{Results}

\section{Grasping force feedback}

Percentages correct grasping forces and percentages grasping forces deviating maximally one force level from the required force were calculated and presented in Figure 2a. Repeated measures ANOVA was performed to evaluate the differences in grasping performance between the two vibrotactile feedback conditions and the control condition without feedback. The percentages correct grasping forces as well as the percentages grasping forces deviating up to one level, differed significantly between the configurations ( $\mathrm{p}<0.001)$. Therefore, Bonferroni corrected ( $\mathrm{p}$-values/3) post-hoc paired samples t-tests were performed to evaluate these differences. No significant differences between both vibrotactile feedback configurations were found ( $p$-values $>0.05$ ), but with vibrotactile feedback the percentages correct grasping forces and percentages grasping forces deviating up to one level were significantly higher compared to the nonfeedback condition ( $p$-values between $\leq 0.001$ and 0.002). No significant differences in task duration between the feedback conditions were found ( $p=0.107)$, which is shown in Figure 2b. 
(a)

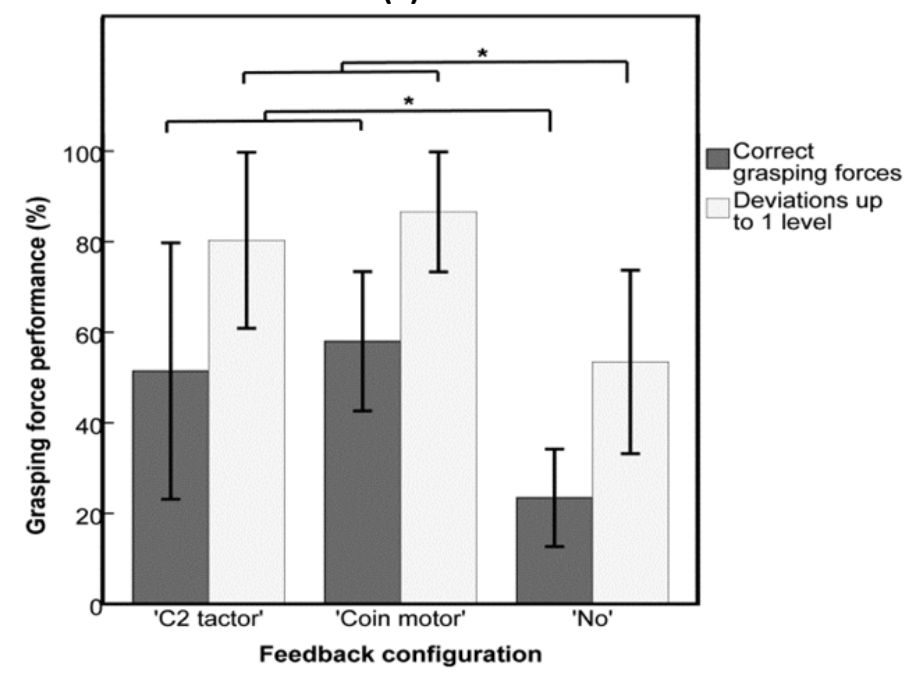

(b)

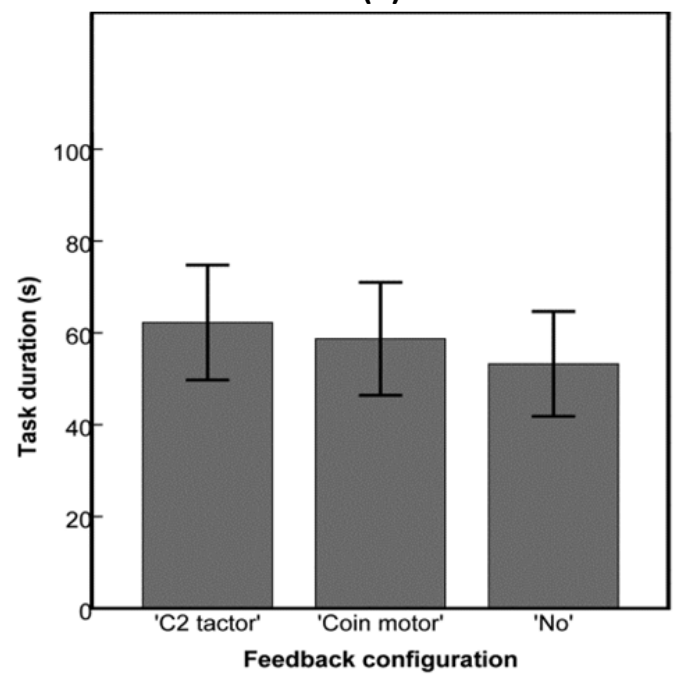

Figure 2: (a) Mean percentages correct grasping forces and percentages grasping forces deviating up to one level, and (b) mean task durations. Standard deviations are indicated by whiskers. Results are given per grasping force feedback configuration. Significant differences $(p<0.05)$ are indicated with an asterisk

\section{Hand aperture feedback}

Mean percentages correct hand apertures and mean percentages hand apertures deviating up to one level from the correct hand aperture were calculated for each feedback configuration and shown in Figure 3a. Configurations were abbreviated according to the visual feedback situation ('YV' for visual feedback and 'NV' for no visual feedback) and the array orientation (' $\mathrm{T}$ ' for transversal and ' $\mathrm{L}$ ' for longitudinal).

Repeated measures ANOVA showed that the percentages correct hand apertures, the percentages hand apertures deviating up to one level and the task durations differed significantly over the five feedback configurations ( $p$-values $<0.001$ ). Bonferroni corrected post-hoc paired samples t-tests were performed to further evaluate these differences. The comparison between the transversal ('NVT') and longitudinal ('NVL') orientation did not show a significant difference for both percentages ( $p$-values $>0.5$ ). For both orientations, the combined visual and vibrotactile feedback condition resulted in significantly higher performance scores compared to vibrotactile feedback only ( $\mathrm{p}$ values $<0.001$ ). Percentages correct hand apertures were significantly higher for the vibrotactile feedback only conditions compared to no feedback ( $\mathrm{p}=0.04$ for both orientations), which was also true for the percentages hand apertures deviating up to one level ( $p=0.008$ for the transversal and $p=0.024$ for the longitudinal orientation).

Task durations did not differ significantly between both array orientations (pvalues $>0.4$ ) and not between the combined visual and vibrotactile feedback and the vibrotactile feedback only conditions ( $p$-values of 1 ). When using vibrotactile feedback only via a transversal oriented array the task duration did not differ from the nonfeedback condition $(\mathrm{p}=0.102)$, but for the longitudinal oriented array a marginal increase in task duration was found $(\mathrm{p}=0.046)$, which is indicated in Figure $3 \mathrm{~b}$. 
(a)

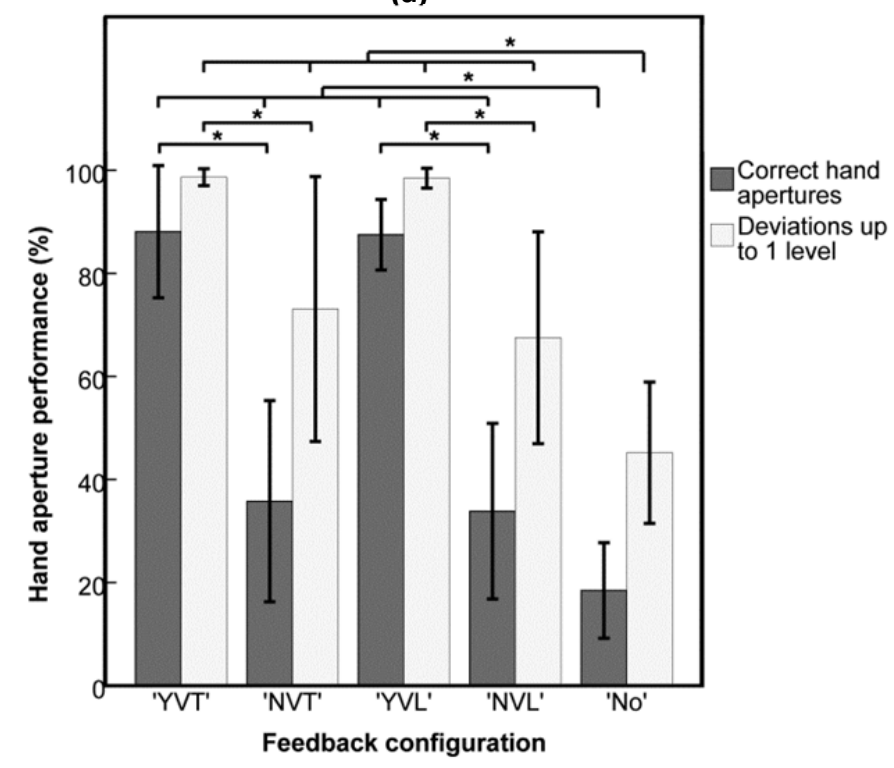

(b)

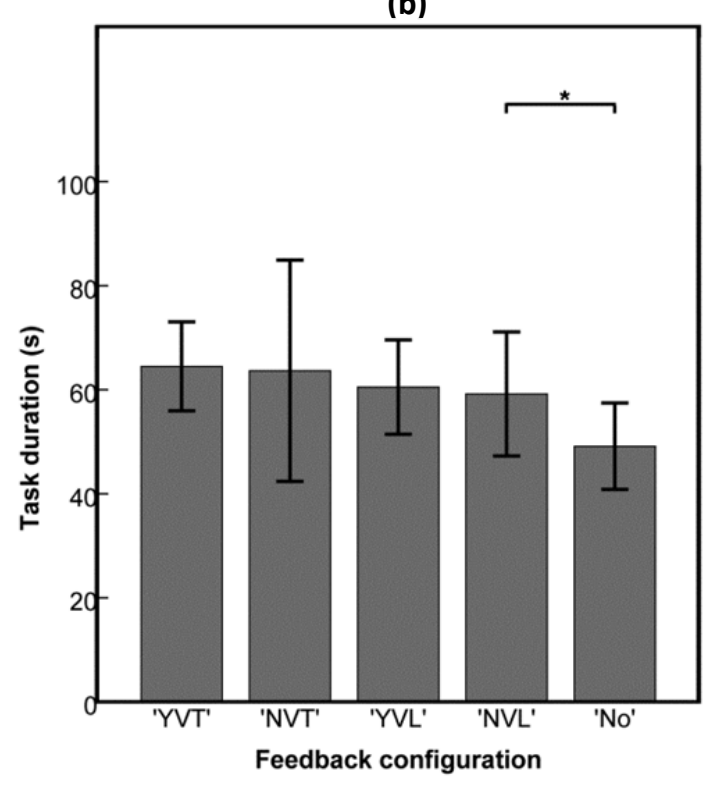

Figure 3: (a) Mean percentages correct hand apertures and percentages hand apertures deviating up to one level, and (b) mean task durations. Standard deviations are indicated by whiskers. Results are given per hand aperture feedback configuration. 'YVT' = visual feedback and transversal array orientation 'NVT' = no visual feedback and transversal array orientation, ' $Y V L$ ' = visual feedback and longitudinal array orientation ' $N V L$ ' = no visual feedback longitudinal array orientation, ' $\mathrm{No}^{\prime}=$ no visual and no vibrotactile feedback. Significant differences $(\mathrm{p}<0.05)$ are indicated with an asterisk

\section{Comparison with healthy subjects}

The results from both studies on healthy subjects $[157,159]$ were combined with the results from this study and presented in Figure 4 and Figure 5. Unpaired samples t-tests showed that the percentage correct grasping forces for subjects with upper limb loss using the coin motor configuration was significantly lower $(\mathrm{p}=0.011)$ compared to the healthy subjects, which was the only significant difference for the grasping force feedback experiments. When visual feedback combined with vibrotactile hand aperture feedback was available, healthy subjects scored significantly better on all outcome parameters (higher percentages and lower task durations with p-values between 0 and 0.038). Task durations were only significantly higher for subjects with upper limb loss compared to healthy subjects $(\mathrm{p}=0.039)$ when using a longitudinal oriented array. 


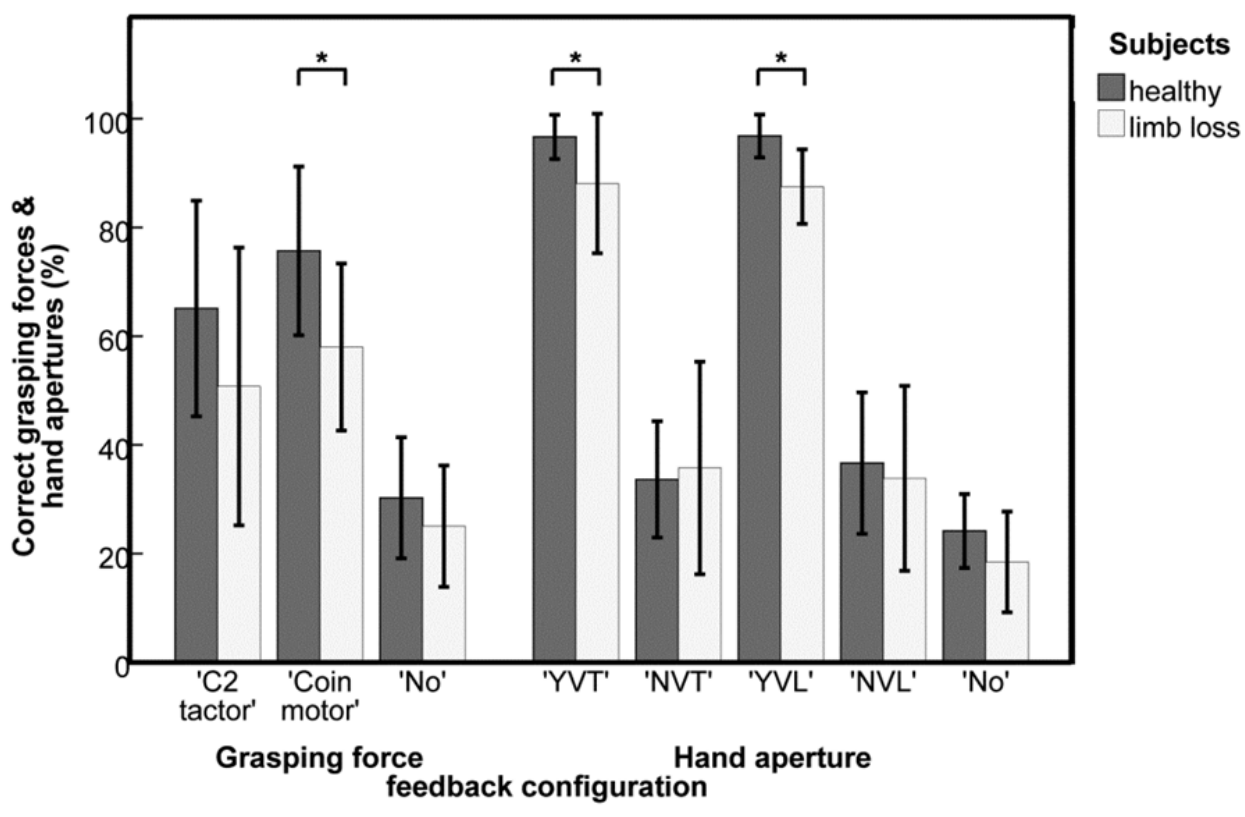

Figure 4: Comparison of percentages correct grasping forces and hand apertures between healthy subjects and subjects with upper limb loss. Means percentages and corresponding standard deviations are given per feedback configurations and significant differences $(p<0.05)$ are indicated with an asterisk

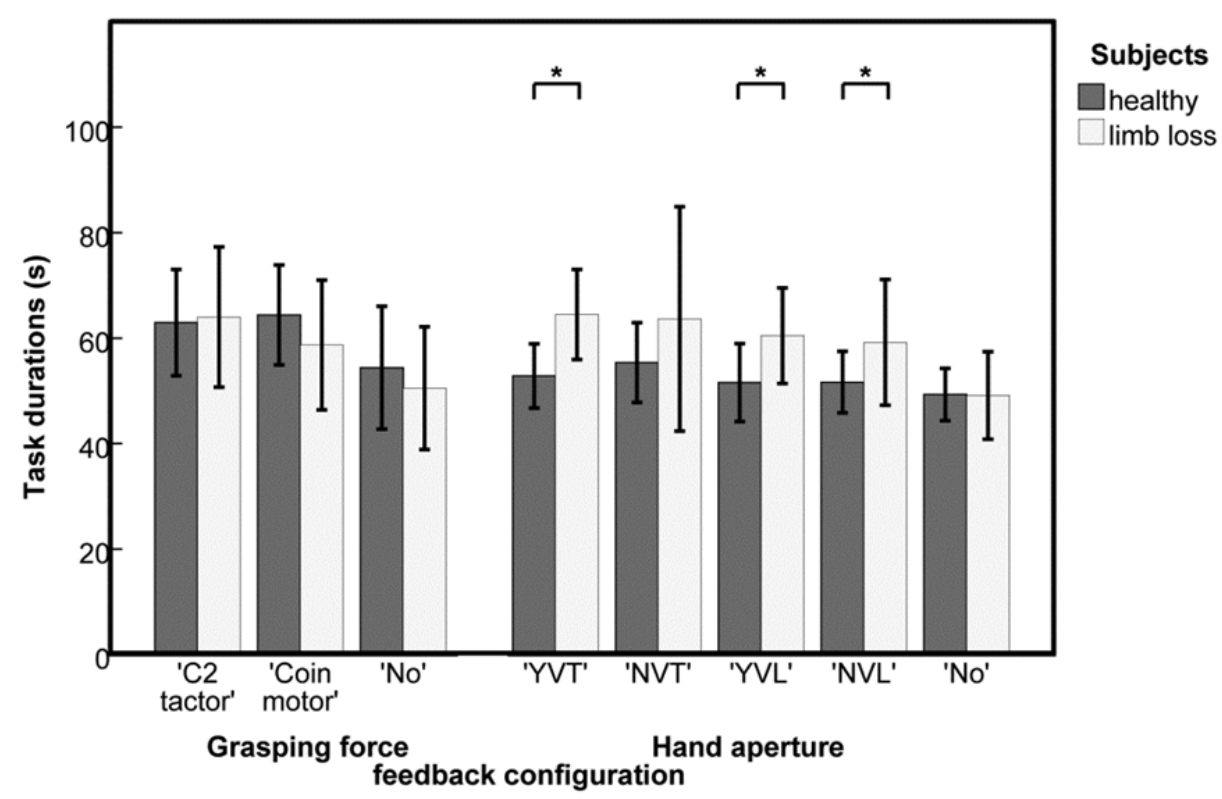

Figure 5: Comparison of task durations between healthy subjects and subjects with upper limb loss. Mean percentages and corresponding standard deviations are given per feedback configurations and significant differences $(p<0.05)$ are indicated with an asterisk 


\section{Discussion}

\section{Grasping force feedback}

The addition of vibrotactile grasping force feedback significantly increased both the percentages correct grasping forces and the percentages grasping forces deviating up to one level compared the non-feedback situations. Therefore, it was concluded that the vibrotactile grasping force feedback could be interpreted by amputees and congenital defect subjects. In literature already positive results with a C2 tactor relating its vibration amplitude to the applied normal force were described [140]. However, this study was performed with healthy subjects, using a completely different setup. Other studies, using frequency modulation of a single coin motor [40], a C2 tactor [28] or an array of coin motors [123], did not show an increase in performance compared to situations without vibrotactile force feedback. None of these studies did include measurements on subjects with upper limb loss. The only study on subjects with upper limb loss, which used a single coin motor with its frequency related to the grasping force, showed better controlled grasping forces [116].

Two grasping force feedback configurations were compared in our study, because no best configuration could be selected based on literature. The $\mathrm{C} 2$ tactor was selected, because it is commonly used in experimental studies and the local increase in amplitude of vibration would be most intuitively related to the localized sensation of pressure at the skin. The array of coin motors was selected, because it showed good results in a previous study on hand aperture feedback and is a relatively cheap feedback method [157]. No significant differences in grasping performance between both configurations were found in our study. It should be investigated in future studies which feedback method can be best combined with hand aperture feedback and the EMG (electromyographic) control of the prosthesis.

\section{Hand aperture feedback}

When only vibrotactile hand aperture feedback was provided, the percentages correct hand apertures were around 35\%, which is not very high, especially compared to the situations with additional visual feedback (percentages of around 90\%). However, the percentages increase to around $75 \%$ for the percentages hand apertures deviating up to one level. Furthermore, even for the percentages correct hand apertures, the results with only vibrotactile feedback were significantly better compared to the non-feedback situations, which shows that vibrotactile hand aperture feedback can be useful in situations where sight on the prosthesis is blocked. Task durations were not significantly increased with vibrotactile feedback only compared to the non-feedback and additional visual feedback situations, which indicates that the interpretation of the vibrotactile feedback did not require significantly more time.

The comparison between the transversal and longitudinal array orientations did not show any significant differences in performance measures, except for a marginally increase in task duration for the longitudinal orientation in comparison to the nonfeedback situation. In studies by Green [62] and Cody et al. [41] better localization 
performances were found for transversal oriented arrays, being explained by the asymmetric shape and orientation of the receptive fields of the mechanoreceptors, with smaller diameters in the transversal direction. However, localization performance can also be increased when stimulators are placed near bony landmarks [35], which was the case for the longitudinal orientation (stimulators over the elbow joint and near the end of the residual limb). Stimulators were placed on the upper arm to realize equal interstimulator distances for both arrays. The mean inter-stimulator distance was around 3 $\mathrm{cm}$, which is just within the range of spatial acuities found for the forearm $(2-3.5 \mathrm{~cm})$ [142], which makes it nearly impossible to reach $100 \%$ scores for the correct hand apertures. The number of stimulators placed on the upper arm depended on the length of the residual limb, but on average 4 stimulators were placed on the upper arm. An advantage of the transversal array is that it can be completely incorporated in the prosthesis, but a disadvantage could be the interference with the electrodes needed for the prosthesis control and the available space in the prosthesis socket, which should be investigated in future studies.

\section{Comparison with healthy subjects}

For the hand aperture feedback experiments, healthy subjects showed higher performance results with visual feedback, which is likely caused by the higher level of computer experience and their experience in participation in experimental studies, while this was totally new for the subjects with upper limb loss. The somewhat higher task durations for subjects with upper limb loss could have been caused by the higher age of the subjects, because reaction times decrease with increasing age [48]. Furthermore, in a study by Stevens and Choo [142] it was shown that the spatial acuity diminishes with aging. However, differences in spatial acuity, caused by the age differences, could also have been counteracted by an improvement in spatial acuity for amputees compared to healthy subjects as presented in other studies $[66,146]$.

Beforehand, it could not be predicted how subjects with upper limb loss would perform in vibrotactile feedback experiments in comparison to healthy subjects, because there is no consensus on possible sensitivity differences. Our study showed that possible differences at least did not influence the usefulness of vibrotactile feedback, because performance results were highly similar to the results of healthy subjects.

\section{Methodological considerations}

To be able to compare the results of the current study with results from the measurements on healthy subjects, the same virtual setup was used. A virtual setup was used to block the natural sensory pathways of the healthy subjects. The 'clicks' from the mouse scroll were removed and the gain relating the mouse scrolling to hand aperture or grasping force was randomized to eliminate clues about the hand aperture or grasping force. The success of this method was represented in the low percentages correct hand apertures and grasping forces in the non-feedback situations, which were around guessing level. 
In this study, the virtual hand was controlled by the sound arm, while feedback was provided to the residual limb. Mouse scroll control was used to be able to compare the results of this study with previous results on healthy subjects [157, 159]. In these studies, feedback was provided to the same arm as used for the control of the virtual hand, which could have contributed to differences in performance. EMG control of the virtual hand was excluded from these studies, because preliminary tests showed that EMG control required a long training period and resulted in high variability in grasping performance between subjects. However, in future studies, with a shorter protocol and a longer training period, it should be investigated whether EMG control influences the interpretation of the vibrotactile feedback. Furthermore, it is preferable to evaluate the feedback concepts in real-life grasping tasks with upper limb loss subjects using a myoelectric prosthesis.

\section{Conclusion}

Vibrotactile grasping force feedback and hand aperture feedback both improve the performance of subjects with upper limb loss in virtual grasping tasks, expressed in higher percentages correct grasping forces and correct hand apertures. No significant differences in grasping performance were found between the feedback configurations. It should be evaluated in future studies, which feedback configurations are optimal when combining both feedback methods in one system and performing daily life grasping tasks with a real myoelectric prosthesis. 
8- Vibrotactile feedback for amputees

$\underset{\substack{\frac{9}{0} \\ \frac{0}{0} \\ \frac{0}{0} \\ \infty}}{\infty}$ 
Chapter 9 - Daily life grasping performance with vibrotactile feedback

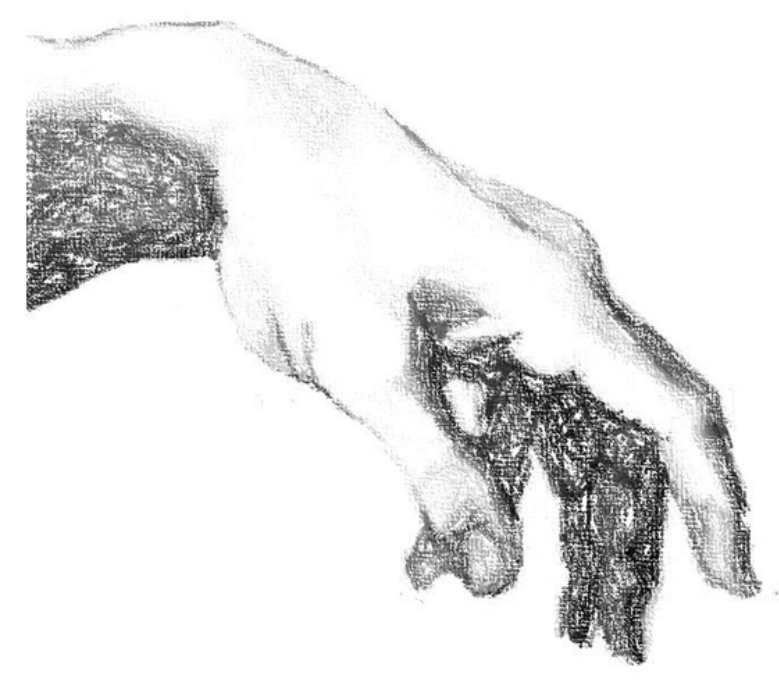

Daily life grasping tasks performed with a myoelectric forearm prosthesis in combination with vibrotactile hand aperture and grasping force feedback Authors: Heidi Witteveen, Simone Fricke, Hans Rietman, Peter Veltink 


\section{Abstract}

In several studies vibrotactile stimulation has been proposed to provide sensory feedback about grasping force and hand aperture, which is essential in object handling with a myoelectric forearm prosthesis. However, the combination of vibrotactile grasping force and hand aperture feedback has not yet been evaluated in daily life grasping tasks performed with a myoelectric forearm prosthesis. In this study 10 healthy subjects performed three different grasping tasks: (1) an abstract grasping task in which object characteristics had to be distinguished, (2) a grape transfer task and (3) the SHAP test, which consists of the transfer of abstract objects and daily life grasping tasks. The tasks were performed with a myoelectric forearm prosthesis, which was connected to the healthy arm and controlled by EMG from the forearm muscles. Tasks were performed under different feedback conditions: with and without vibrotactile feedback and with and without visual feedback. Grasping force feedback was provided through amplitude modulation of a single C2 tactor and hand aperture feedback through an array of 8 coin motors placed around the forearm. Percentages correctly identified object sizes and stiffness were significantly increased with the use of vibrotactile feedback compared to the no feedback situation, but did not reach the levels of the visual feedback conditions. No differences in performance in the grape transfer task and the SHAP test were found between the vibrotactile feedback and the visual feedback only condition. However, most of the subjects perceived the vibrotactile feedback as being helpful in these grasping tasks as well. The addition of vibrotactile feedback about grasping force and hand aperture improved object identification in cases of blocked vision compared to no feedback, but it takes time to interpret the feedback. 


\section{Introduction}

Missing a hand due to amputation or a congenital defect always negatively influences the functioning in daily activities, which can be partly compensated for by a forearm prosthesis. Current myoelectric prostheses offer the users improved functionality by increasing the number of possible grasps, individual finger movements and wrist movements. However, in relation to the total number of potential users, the percentage myoelectric prosthesis users is low $[12,45]$ and it is also a small percentage of this group that is using their myoelectric prosthesis on a regular and daily basis. Even 20$34 \%$ of the users once fitted with a myoelectric prosthesis is not using the prosthesis at all $[18,45]$. Besides the perceived heavy weight and limited functionality of the prosthesis, the lack of sensory feedback has been indicated as a major shortcoming of today's myoelectric prostheses [12,18].

Via questionnaires filled out by myoelectric prosthesis users [86] and a workshop on representative prosthesis users [107], feedback requirements were derived. Grasping force feedback has been indicated as the most important option to include, because force information cannot be derived visually. Besides grasping force feedback, hand aperture feedback has also been indicated to be important, because this will reduce the burden on the visual system and enables use of the prosthesis in situations where sight on the prosthesis is blocked.

An increasing number of studies is being performed on the development of artificial sensory feedback methods, focusing mostly on non-invasive methods. Both electrotactile $[16,115,127]$ and vibrotactile stimulation have been investigated to provide grasping force feedback. Vibrotactile stimulation has been provided either through a single C2 tactor (linear vibrotactile transducer) $[28,40,140]$, a single coin motor $[113,116]$ or an array of coin motors [123]. Except for an early study using electrotactile stimulation [114], a study using a single C2 tactor [28] and a study comparing the use of an array of vibrotactile and electrotactile stimulators [157], no other studies have been found on feedback about the hand aperture of a prosthesis. In two of the mentioned hand aperture feedback studies [28, 114], hand aperture feedback is combined with grasping force feedback in one system. They showed that it was hard to distinguish between hand aperture and grasping force feedback when using one stimulator for both modalities [114] or they did not evaluate the effect of the hand aperture feedback [28]. The combination of hand aperture feedback and grasping force feedback can also provide information about the stiffness of an object, which is important in object handling as well, because it cannot be derived visually and helps in object discrimination 136]. A previous study has shown that vibrotactile hand aperture feedback in combination with grasping force feedback is indeed helpful in stiffness discrimination in virtual grasping tasks [158].

Most of the above described studies have not been performed in real life grasping tasks performed with a myoelectric prosthesis. In a study by Pylatiuk et al. the performance in an object lifting task, while receiving grasping force feedback through a single coin motor, was evaluated[116]. They found a decrease in applied grasping force when force feedback was provided. In the earlier described study of Chatterjee et al. [28] subjects 
were asked to provide three levels of grasping force while holding a ball and receiving feedback about the grasping force through pulse rate modulation of a C2 tactor. The prototype Smarthand prosthesis has been evaluated during functional grasping tasks by Cipriani et al. [40]. Vibrotactile feedback about the grasping force did not significantly improve the grasping performance when visual feedback was also available, but subjects did report that the feedback was helpful.

In this study the combination of vibrotactile hand aperture and grasping force feedback is evaluated, while performing varying grasping tasks using a myoelectric prosthesis. Three different grasping tasks ranging from grasping of abstract objects to activities of daily living were evaluated.

\section{Methods}

\section{Subjects}

Two experiments were performed in this study, each involving a different group of 10 healthy subjects. The first group consisted of 4 males and 6 females $(20.5 \pm 1.8$ yrs.), the second group of 7 males and 3 females (26.8 \pm 2.4 yrs.). The mean forearm length was $26.3 \pm 2.3 \mathrm{~cm}$ and the mean forearm circumference was $27.1 \pm 2 \mathrm{~cm}$. One subject was excluded from the second group of subjects, because he was not able to successfully control the measurement setup, which was concluded during the training phase of this study.

All subjects were informed about this study via an information letter and all signed informed consent forms preceding the experiments. The study protocol has been approved by the local medical ethical committee (Medisch Ethische ToetsingsCommissie Twente).

\section{Materials}

An Otto Bock proportional DMC hand prosthesis (Otto Bock HealthCare GmbH, Duderstadt, Germany) was used during the experiments. This hand provides proportional control of the movement velocity and the grasping force. The movement velocity during hand opening and closing and grasping force during object holding are linearly related to the amplitude of the EMG signal. Accompanying EMG electrodes (Myobock electrodes) were used to derive the EMG control signal from the wrist flexors and wrist extensors. To attach the prosthetic hand to the forearm of the subjects a custom-built aluminum frame was used (see Figure 1), which was secured to the arm by Velcro straps. The wrist of the subjects was immobilized by a wrist brace to realize isometric contractions.

A wetsuit sleeve was used to incorporate the vibrotactile stimulators and the EMG electrodes and fit them around the forearm. The location of the EMG electrodes was derived by palpation of the wrist flexors and extensors of the forearm and the EMG electrodes were fixed to the correct location in the wetsuit sleeve by Velcro straps. 


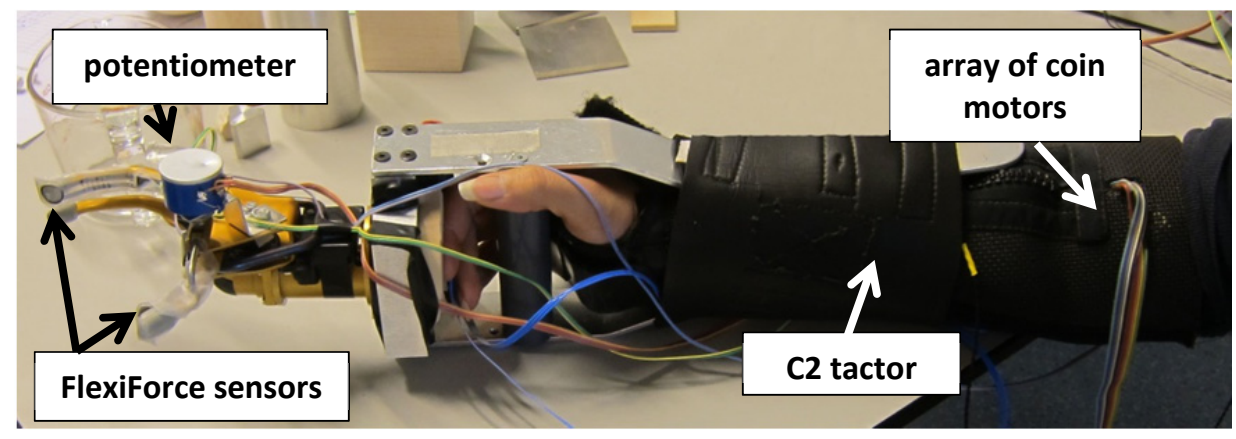

Figure 1: Overview of the prosthetic hand connected to the healthy arm via an aluminum frame. The C2 tactor, array of coin motors and EMG electrodes were incorporated in the wetsuit sleeve and a wrist brace was used to immobilize the wrist. A potentiometer and FlexiForce sensors were added to the prosthetic hand to measure the hand aperture and grasping force respectively

A C2 tactor (miniature vibrotactile transducer) was used to provide the grasping force feedback. Grasping force was measured by two force sensors (FlexiForce sensors, Tekscan Inc., South Boston, USA) placed at the fingertip of the index finger and the thumb. During grasping, these points are in contact with the objects. The range from no grasping force to the maximum summed grasping force measured from both sensors was divided into eight discrete levels. These eight force levels were related to eight amplitude levels of the $\mathrm{C} 2$ tactor, ranging from 0 to $5 \mathrm{~V}$ with equal intervals. The $\mathrm{C} 2$ tactor was placed in the sleeve such that it was located halfway the dorsal side of the forearm.

The hand aperture was measured by a potentiometer $(10 \mathrm{k} \Omega$ potentiometer, MCB Industrie, Chateau Gontier, France) placed above the rotation point of the thumb (see Figure 1). The range between the fully opened and closed hand was divided into eight discrete hand aperture levels. Each hand aperture level was related to activation of a single coin motor. Eight coin motors were placed within the sleeve and socket such that they were equally distributed circular around the forearm, $3 \mathrm{~cm}$ distal from the elbow joint.

\section{Protocol}

Subjects were comfortably seated behind a table in a silent room. The height of the chair was adjusted such that the arm of the subjects rested on the table with an elbow angle of around 90 degrees. The objects to be grasped were all placed on the table within reaching distance from the subject.

Three different tasks were evaluated in this study: (1) an abstract grasping task (object discrimination performed with a myoelectric forearm prosthesis to evaluate the hand aperture and grasping force feedback methods; (2) a functional grasping and lifting task to evaluate whether the handling of fragile objects will be improved with feedback and (3) a standardized test, the SHAP [88], which is developed for the evaluation of grasping performance with a forearm prosthesis and in this study used for evaluation of the effect of the vibrotactile feedback in real life grasping tasks. The abstract grasping task was performed by the first group of 10 subjects. The fragile objects task and the SHAP test 
were performed by the second group of 10 subjects. This last group of subjects also performed the abstract grasping task under the control condition without visual and vibrotactile feedback. This control condition was added to evaluate whether vibrotactile feedback is of additional value and whether subjects could use other sources of feedback like the motor noise of the prosthesis.

Before the execution of the tasks, a training procedure was incorporated to ensure that the subjects were able to adequately control the prosthesis and interpret the feedback. A custom-made Labview program was used to show on the computer screen the actual discrete hand aperture or grasping force level together with the required grasping force or hand aperture. Subjects were asked to reach the required hand aperture or grasping force within five seconds. Subjects were judged as being successful in prosthesis control if they were able to reach the required level within a range of \pm 1 level five times in a row successfully. Afterwards, subjects were asked to perform the same tasks, but now feedback about the hand aperture or grasping force was provided simultaneously. Subjects were asked to concentrate on the feedback and they were considered successful if they could reach the required level of hand aperture or grasping force within a range of \pm 1 level ten times in a row. The last training round consisted of the same tasks, but now the actual hand aperture or grasping force was not shown on the screen and a box was placed over the prosthesis hand. In this way, the interpretation of the vibrotactile feedback was evaluated and trained.

The vibrotactile feedback that was provided in all three tasks consisted of simultaneously provided feedback about the grasping force and hand aperture of the prosthesis trough activation of the $\mathrm{C} 2$ tactor and the array of coin motors respectively. In conditions with only vibrotactile feedback, the visual feedback was blocked by placing a box over the hand and the forearm, blocking the sight on the prosthesis. The order of the execution of the three tasks and the order of the feedback conditions was randomized to avoid learning effects with the prosthesis and the feedback. After finishing a task, under all feedback conditions, the subjects were asked to score the perceived helpfulness of the vibrotactile feedback in performing the grasping tasks on a VAS scale (line of $10 \mathrm{~cm}$ ) ranging from 'not helpful at all' to 'very helpful'.

\section{Abstract grasping task}

For the abstract grasping task, cylindrical objects were made by 3D-printing. Three objects with different lengths $(6,7.5$ and $9 \mathrm{~cm})$, but similar diameters, were constructed (see Figure 2a). A fully opened hand was required to hold the largest object and a halfway closed hand to hold the smallest object. The weight of the object was varied by placing pieces of lead slab in the cylinder, which resulted in object weights of 70, 140 and 210 grams (see Figure 2b). Furthermore, a spring was placed in between the two moving parts of the cylinder to give the object a certain stiffness (see Figure 2b). Three different stiffness levels were created by using a compliant spring (requiring only the first level of grasping force to compress), a stiff spring (requiring more force, around the fifth level, to compress) and an incompressible piece of plastic. Both the stiffness and weight of the object could not be visually derived from the outside of the object. 
(a)

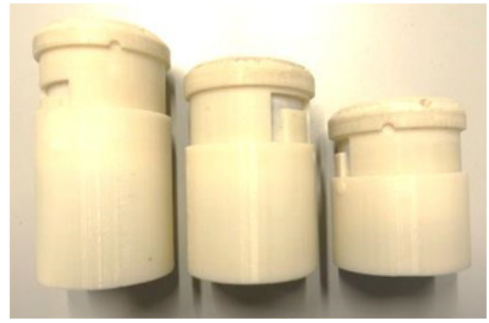

(b)

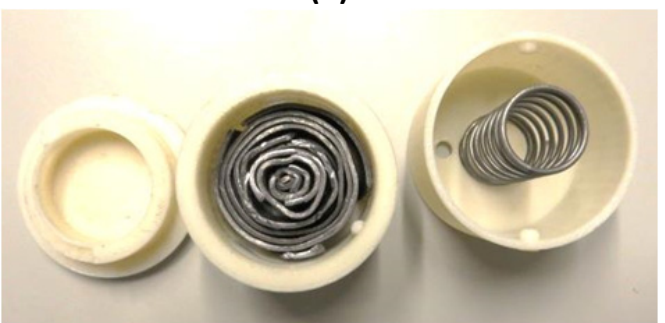

(c)

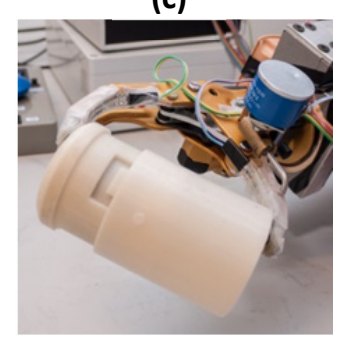

Figure 2: (a) The three object sizes, (b) from left to right: the lid of the object, the weight (lead slab) inside the object and the spring, (c) the object placed in between the prosthetic hand

The objects were placed horizontally in between the prosthetic hand, such that the weight of the object did not influence the measured grasping force. To check whether the behavior of the objects in combination with the different springs was the same for each object size and weight, displacements, measured by the potentiometer, were plotted against the applied force during compression of the objects. It was shown that for the two smallest objects the relation between the applied force and the resulting displacement was linear as expected. However, for the largest objects more force was required at the start of the movement, resulting in deviations from the linear relation. These problems occurred when the fingers could not be placed exactly in the middle of the object. The relationships between applied force and resulting displacements for the three different springs could be visually distinguished for all three object sizes and weights and therefore it is expected that object stiffness can be identified through the hand aperture and grasping force feedback.

The weight, stiffness and size of the objects was randomized during the grasping tasks, but it was ensured that each object characteristic was used 5 times on a total of 15 objects. An object with a selected weight, size and stiffness was presented in between the opened prosthesis hand by the experimenter and the subjects were asked to grasp and lift the object (see Figure 2c). The experimenter ensured that the fingertips of the prosthesis were at the middle of the object and that the object was held horizontally. Subjects were allowed to grasp and squeeze the objects as often as they needed. When they were certain about the object characteristics the time was stopped and the subjects were asked to indicate the characteristics (stiffness, weight and size) of the object. Three feedback conditions were evaluated: 1) only visual feedback, 2) visual feedback and vibrotactile feedback and 3) only vibrotactile feedback. The control condition without vibrotactile and without visual feedback was performed by the second group of subjects. This control condition was added to evaluate how much information about grasping force and hand aperture of the prosthesis could be derived for example from the noise of the motor. The outcome parameters for the abstract grasping task were the task duration and the percentages correctly identified objects per object characteristic. 


\section{Grape transfer task}

The grape transfer task consisted of 20 grapes (white grapes with varying sizes) that had to be grasped and transferred by the subjects. Grapes were chosen, because these objects have been used more often to prove that fragile objects can be grasped with a prosthetic hand. Preliminary tests showed that the third grasping force level $(1 / 3$ of the grasping force range) was the maximal grasping force before squeezing the grapes. The objects were placed on the left side of the table and the subjects were asked to grasp the grapes one by one and drop the grape at the right side of the table (see Figure 3). The task durations were derived from a large stopwatch that was pressed by the subjects at the start and end of the tasks. The number of successfully transferred grapes (without squeezing) was scored by the experimenter. The grape transfer task was performed for two feedback conditions: (1) visual feedback only and (2) visual feedback in combination with vibrotactile feedback. The outcome parameters for this task were the task duration and the percentage of successful grape transfers.

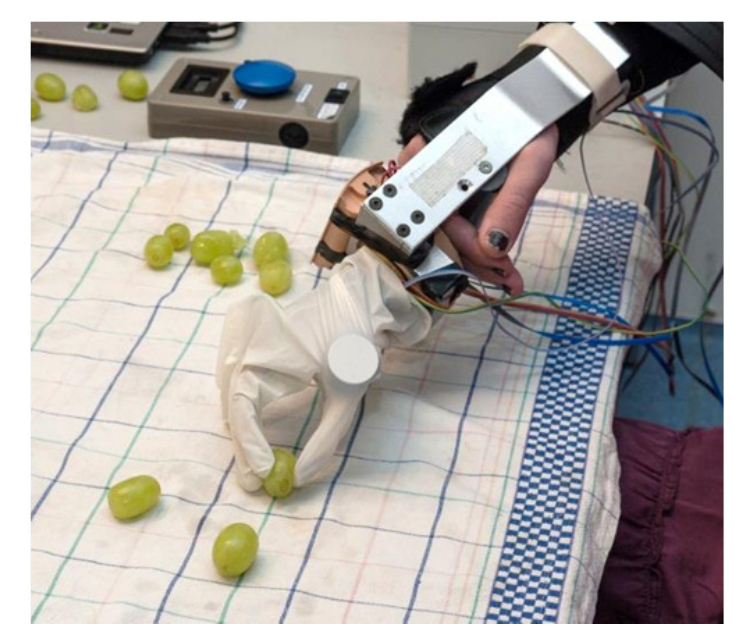

Figure 3: Subject performing the grape transfer task. Grapes had to be transferred from the left to the right side of the table. Task durations were scored by the large stopwatch (blue button)

\section{SHAP test}

The SHAP test is a standardized test for the evaluation of forearm prosthesis use [88]. The protocol of this test must be followed strictly and consists of grasping 12 abstract objects and performing 14 activities of daily living (see Figure 4). The task durations are scored by asking the subjects to press a button at the start and end of the grasping tasks. It was not possible to perform all of the tasks of the SHAP test with the bare prosthetic hand, because some objects slipped out the hand or tasks could not be performed due to the extreme length of the arm with the prosthesis. The tasks that were skipped were: the lifting of the heavy spherical object and the heavy lateral object and the button board, the simulated food cutting, the glass jug pouring, the lifting a tray, the open/close zip and the rotate a screw tasks. The remaining 10 abstract tasks were: the lifting of the light and heavy tripod, power, tip and extension object and the lifting of the light spherical an lateral object. The remaining 8 daily life grasping tasks were: the pick-up coins, page turning, jar lid, carton pouring, lifting a heavy and light object, rotate key and 
the door handle task. The SHAP test was performed for two feedback conditions: (1) visual feedback only and (2) visual feedback in combination with vibrotactile feedback. For all the skipped tasks, task durations of $100 \mathrm{sec}$. were scored, according to the SHAP protocol for tasks that could not be performed by the subject. The outcome parameters for the SHAP test were the task durations and the IoF (Index of Functionality) scores derived from the SHAP website after importing all task durations.

(a)

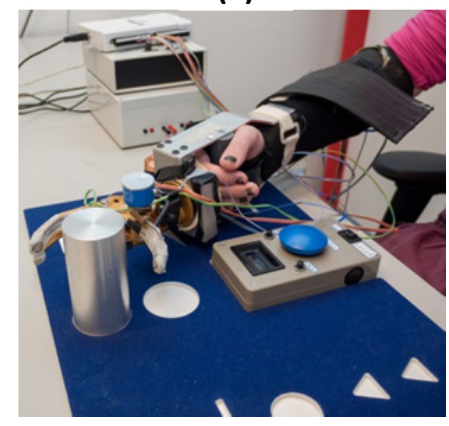

(b)

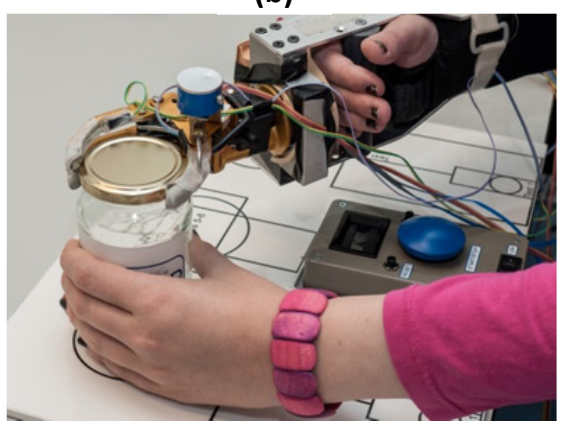

Figure 4: Subject performing the SHAP test; (a) transferring an abstract object and (b) performing a daily life task. Task durations were scored by the subject by using the stopwatch (blue button)

\section{Results}

\section{Abstract grasping task}

Object identification results were analyzed for all three object characteristics separately. In the next figures (Figure 5a-d), the percentages correctly identified object characteristics and the task durations are shown per feedback condition.

A repeated measures ANOVA was performed, using a General Linear Model (GLM) in SPSS, with the three feedback conditions (visual, visual+vibrotactile and vibrotactile) as factor and a significance level of 0.05. This analysis showed that for the task durations, the percentages correctly identified sizes and the percentages correctly identified stiffness a significant influence of the feedback condition exists (all p-values<0.001). No differences between feedback conditions were found for the identification of the weight of the object ( $\mathrm{p}=0.973$ ). On average, the weight of the object was correctly identified in $46 \%$ of the cases. To evaluate possible differences in task durations and identification of stiffness and size between the three feedback conditions, post-hoc Bonferroni corrected paired-samples t-tests were performed. These tests revealed that identification of size and stiffness was significantly better for both visual feedback conditions compared to vibrotactile feedback alone ( $p$-values between $\leq 0.001$ and 0.003 ) and task durations were significantly lower for both visual feedback conditions in comparison to the vibrotactile feedback alone condition ( $p$-values 0.003 - 0.009). No differences between both visual feedback conditions (with and without vibrotactile feedback) were found for the size and stiffness identification and the task durations ( $\mathrm{p}$-values between 0.384 and $1)$. 
(a)

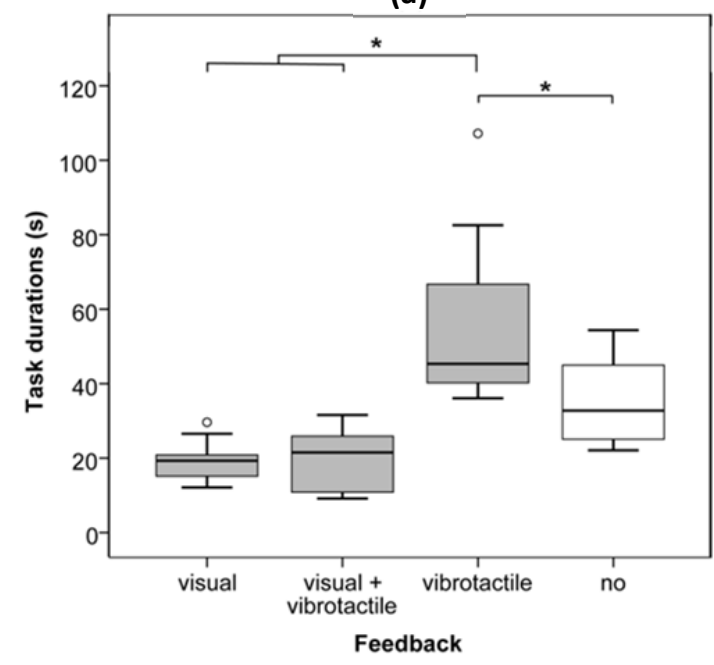

(c)

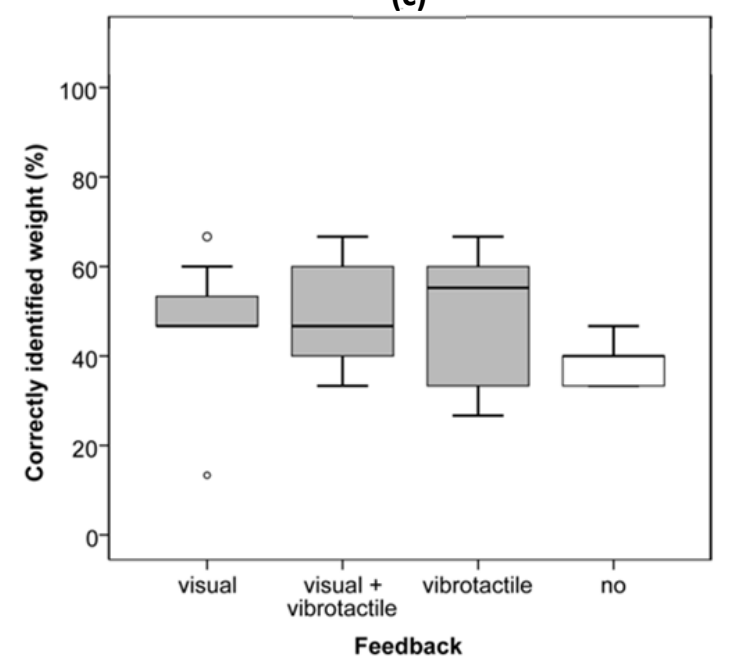

(b)

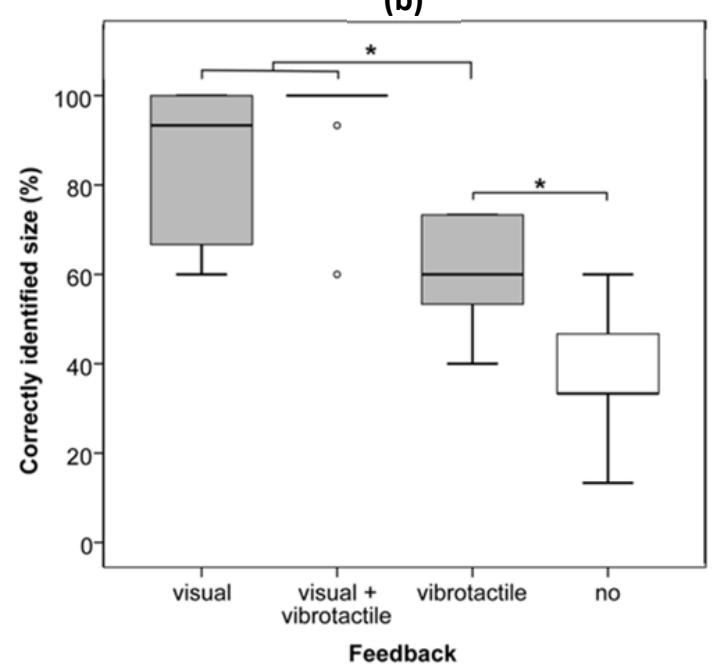

(d)

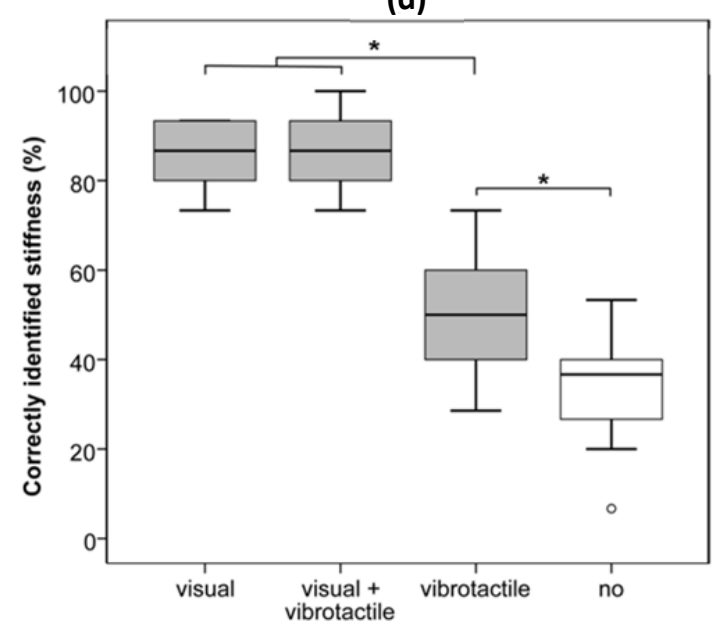

Feedback

Figure 5: Boxplots showing (a) the task durations, (b) percentages correctly identified sizes, (c) percentages correctly identified weights and (d) percentages correctly identified stiffness of the abstract grasping tasks for all three feedback conditions and the control condition without feedback. The upper and lower borders of the boxes indicate the 75 and 25 percentiles respectively and the thicker horizontal line in the box represents the median of the results. The whiskers indicate the minimum and maximum values and outliers are indicated by the open circles

Identification performances are compared between the vibrotactile feedback alone and the control condition without any feedback by unpaired samples t-tests, because the results were derived from two subject populations. No differences in weight discrimination were found $(\mathrm{p}=0.061)$, but with vibrotactile feedback the size and stiffness discrimination was significantly better in comparison to the no feedback condition ( $p=0.002$ and $p=0.023$ respectively) and task durations were significantly increased when using vibrotactile feedback $(p=0.031)$. 


\section{Grape transfer task}

The number of successful transferred grapes and task durations were derived for each subject and visualized in Figure 6 and Figure 7 respectively. A separation was made between both orders of feedback conditions during the measurements to evaluate the learning effect over time.

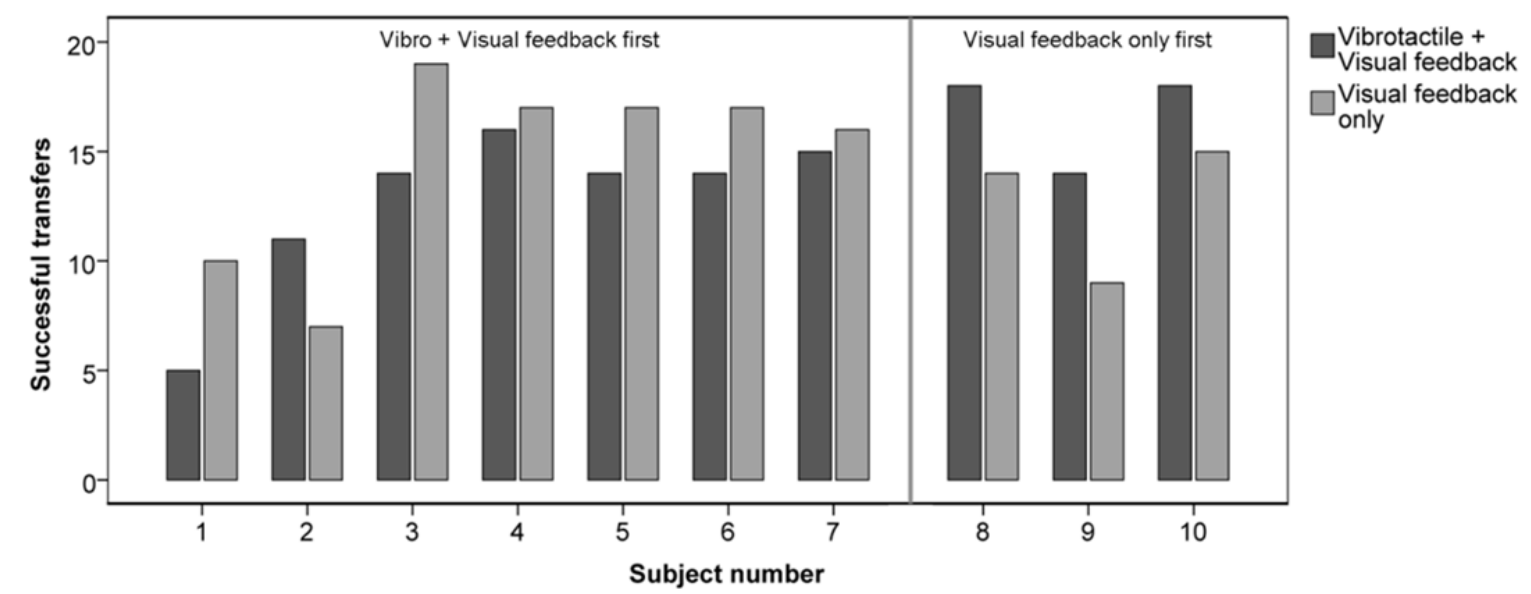

Figure 6: The number of successful transfers for each subject separately, given per feedback condition. The first 7 subjects started with the task under the vibrotactile and visual feedback condition and the latter 3 started with the visual feedback only condition

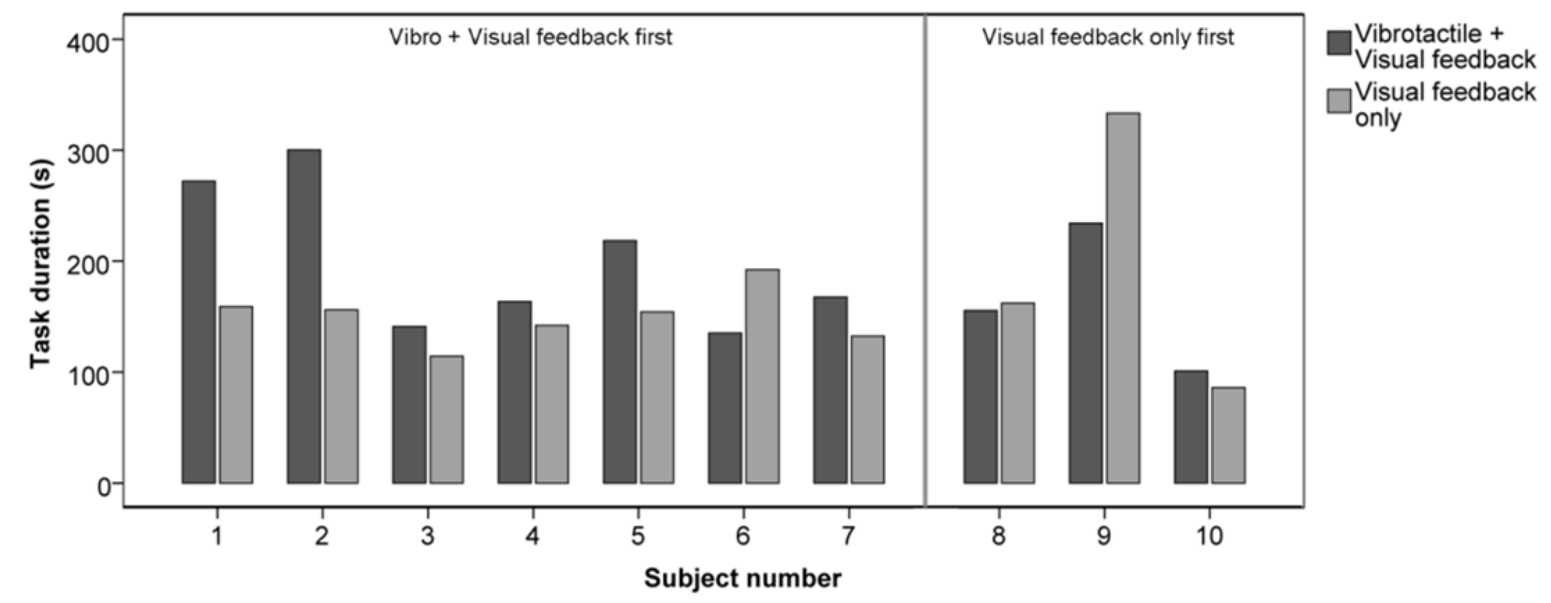

Figure 7: The task durations of the grape transfer task for each subject separately, given per feedback condition. The first 7 subjects started with the tasks under the vibrotactile and visual feedback condition and the latter 3 started with the visual feedback only condition.

Repeated measures ANOVA was performed, using a General Linear Model in SPSS, to analyze the differences in successful transfers and task durations between both feedback conditions. The analysis without covariates showed no differences in successful transfers and task duration between both feedback conditions $(p=0.874$ and 0.289 respectively). However, when introducing the order of the measurements as a covariate in the model, a significant influence of the feedback on the number of successful transfers was found $(p=0.012)$ with lower scores for the vibrotactile feedback 
condition. Furthermore, a significant influence of the order of the measurements was found ( $\mathrm{p}=0.044)$, which was not seen for the task durations.

\section{SHAP test}

Mean task durations were calculated by averaging the task durations of the 18 grasping tasks of the SHAP test and are shown in the next figure (Figure 8a). Furthermore, the Index of Functionality scores were derived by entering the task durations on the website of the SHAP test (see Figure 8b). As a large number of tasks from the original test were excluded in this study, the scores are very low and therefore no comparisons with other studies were made.

(a)

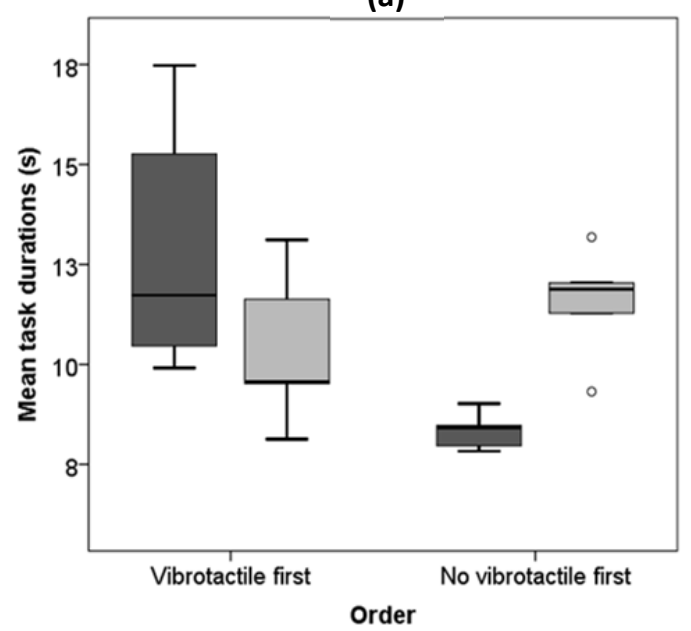

(b)

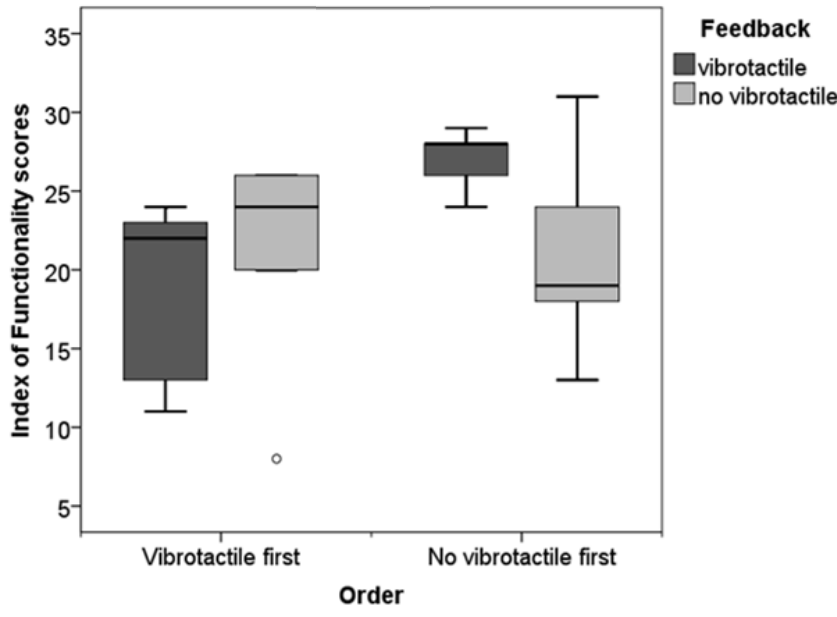

Figure 8: Boxplots showing (a) the mean task durations of the SHAP tests and (b) the derived Index of Functionality scores for both feedback conditions and categorized by the order of the measurements. The upper and lower borders of the boxes indicate the 75 and 25 percentiles respectively and the thicker horizontal line in the box represents the median of the results. The whiskers indicate the minimum and maximum values and outliers are indicated by the open circles

A repeated measures ANOVA test, using a General Linear Model, was performed to analyze the differences in task durations and IoF scores between both feedback conditions. No significant differences in feedback conditions were found $(p=0.781$ for the task durations and $\mathrm{p}=0.412$ for the IoF scores). However, when the measurement order was added as a covariate in the General Linear Model, a significant effect of the measurement order on the task durations was found $(\mathrm{p}=0.002)$, with lower task durations for the vibrotactile feedback condition $(p=0.026)$. No effect of measurement order on the IoF scores was found $(\mathrm{p}=0.053)$.

\section{Subjective experiences}

The additional value of vibrotactile feedback was subjectively evaluated by asking the subjects to indicate the perceived helpfulness on a VAS scale. For all three tests, the VAS scores were scored after completion of the measurements under both conditions. The results are shown in the next figure (Figure 9). 


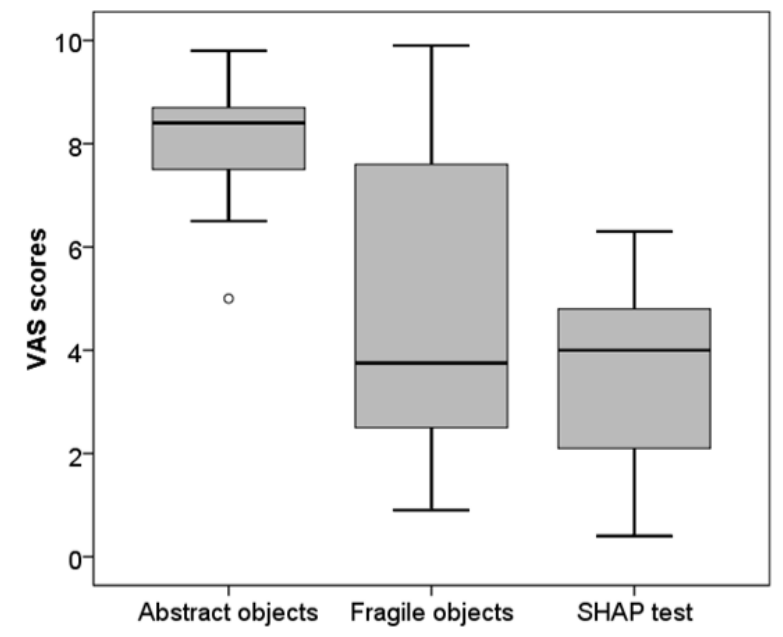

Figure 9: Boxplots showing the distribution of the VAS scores over all 10 subjects for all three experiments. The upper and lower borders of the boxes indicate the 75 and 25 percentiles respectively and the thicker horizontal line in the box represents the median of the results. The whiskers indicate the minimum and maximum values and outliers are indicated by the open circles

\section{Discussion}

\section{Abstract grasping tasks}

Object identification performances were compared between four feedback conditions. The visual feedback only condition was introduced, because it represents the current situation with myoelectric forearm prostheses. Vibrotactile feedback in combination with visual feedback was used to investigate whether vibrotactile feedback is of additional value, which was expected to be the case in weight and stiffness discrimination, because grasping force information cannot be derived visually. However, our results showed no differences between the visual feedback only condition and the combination of visual and vibrotactile feedback. The vibrotactile feedback only condition was introduced to represent situations in which visual feedback is blocked. To evaluate whether subjects were able to use auditory or other information from the prosthesis for object identification, a control condition without vibrotactile and visual feedback was introduced. Other sources of useful information were ruled out by our results, because mean identification performances for object size and stiffness in the no feedback (control) condition were 34 and $37.3 \%$ respectively, which is around the guessing level for three choices in object characteristics.

Subjects were allowed to lift the objects to determine the weight of the objects, using propriocepsis in the arm. However, percentages correctly identified weights were relatively low (around 50\%). This is likely caused by the heavy weight of the prosthesis (944 grams in total) in comparison to the weights of the objects (70, 140 and 210 grams). Furthermore, no differences in weight discrimination performance were found between the different feedback conditions. Beforehand it was expected that grasping force feedback would provide additional information about the weight of the objects, because larger grasping forces are required to hold the heavier objects. However, this 
was not always true for the used objects, because the lowest grasping force level could also be enough to hold the object, due to the roughness of the object. Although grasping force feedback could not be used for weight identification, it did provide touch feedback at the moment an object was touched by the prosthesis, which could be used during the identification of the size of the object.

For object size and stiffness discrimination a significant increase in identification performance was found when using vibrotactile feedback in comparison to no feedback. However, these values did not approach the values when visual feedback could be used and therefore it can be concluded that vibrotactile feedback is of additional value only when visual feedback is blocked. This was also seen in the evaluation study of Cipriani et $a l$. [40]. One of the main aims of (vibrotactile) feedback is to reduce the required visual attention during object handling, but based on these results it cannot be concluded if this aim can be achieved. Percentages correctly identified sizes (around 60\%) with vibrotactile feedback only were comparable with results from our earlier studies using a virtual setup [157], while the percentages for stiffness identification (around 55\%) were a little lower than our previous results [158].

Task durations were significantly increased with vibrotactile feedback compared to the no feedback and the visual feedback conditions. Whether this increase in task durations is acceptable should be further investigated in future studies, but it can also be expected that task durations will decrease with training as was already seen in other studies on vibrotactile feedback $[119,138]$. However, the benefit of the feedback should already be clear for the users from the first start to avoid early abandonment of the prosthesis.

\section{Grape transfer task}

It was expected beforehand that the grasping force feedback would be especially of additional value in handling of fragile objects in daily life. Indication of touch of the object would stop the closing of the hand and information about the grasping force would avoid applying too much force and therefore prevent from squeezing the object. Grapes were used as fragile objects, because preliminary tests showed that the minimal grasping force needed to hold the grapes could be detected by the force sensors, which for example was not possible for plastic cups. No differences between the visual feedback only and the vibrotactile plus visual feedback conditions were found in the number of successful transferred objects and the task durations. After introducing the order of the measurements in the analysis model it was found that the performance is mainly influenced by the order of the measurements. A longer period of training with the prosthesis likely diminishes this effect. However, this experiment was also conducted to see whether vibrotactile feedback can already have a positive effect on the grasping performing at first use, which would increase the acceptance of the feedback system by the user.

Although no differences in performance were found when adding vibrotactile feedback, the helpfulness of the vibrotactile feedback has been scored by the subjects as moderate (average of 4.9 on the VAS scale). This was also found in grasping tasks with vibrotactile grasping force feedback, evaluated by Cipriani et al. [40]. However, the spread in scores 
was very large, which indicates that for some subjects the feedback was perceived as a real additional value and for some subjects it was not helpful at all. Furthermore, the VAS scores were not correlated with the performance in the grasping task. It should be further investigated with a larger number of subjects whether the feedback can be of additional value for all subjects or a subgroup of users can be made.

\section{SHAP test}

Not all tasks of the SHAP test could be performed with the prosthetic hand used in this study, because some objects slipped out of the hand, which was caused by the removal of the rubber hand cover. The Index of Functionality scores that were calculated are based on the task durations of all tasks in relation to the task durations of healthy subjects [88] and therefore all subjects scored very low and the results could not be compared to other studies. However, the main outcome parameters on which the IoF scores are based, are the task durations, which are also analyzed and compared between feedback conditions. No significant differences in task durations and IoF scores were found between both feedback conditions, but task durations were clearly influenced by the order of the experiments. Introducing the order of the measurements as a covariate, indicated this as well and also showed that task durations were significantly lower with vibrotactile feedback when the task was first performed without vibrotactile feedback. This would imply a strong learning effect that is improved by the vibrotactile feedback. However, these effects should be investigated per measurement order to prove these suggestions, which was not possible, due to the low number of subjects in both groups.

In accordance with the grape transfer task, the subjects experienced the vibrotactile feedback as being moderately helpful, but the spread in scores was lower and none of the subjects scored higher than 6 for the helpfulness of the feedback.

\section{Methodological considerations}

An Otto Bock myoelectric forearm prosthesis was equipped with force sensors and a potentiometer, which disabled the use of a rubber hand cover, which resulted in some problems with the lifting of heavy and slippery objects. Furthermore, the sensors and the vibrotactile elements were all connected to the measurement devices with many long cables, which sometimes hampered the movements of the subjects. Finally, the control of the prosthesis and the handling of objects was also influenced by the weight of the prosthesis and the aluminum frame and the longer length of the prosthesis compared to the normal arm. More daily life tasks could be investigated when the prosthesis control is less interrupted by all the influences mentioned above. In current state-of-the-art myoelectric forearm prosthesis, sensors are already incorporated in the prosthesis, to realize for example automatic slip control, which can possibly also be used for feedback input. To some extent, these prostheses do also provide the control of separate fingers, which likely requires other feedback methods, which should be investigated in future studies.

In this study, simple FlexiForce sensors were used, that are sensitive to drift. To overcome this problem in the present study, the sensors were calibrated before the start 
of each measurement. However, in future studies, other small force sensors could be used, for example those that are already used in current state-of-the-art prostheses or small 3-D force sensors that are currently being developed [25]. These latter sensors can also provide information about the forces in the tangential direction, which might be useful as well.

The abstract objects that were used in this study did have limited weights, sizes and stiffness levels, due to the design of the objects that should fit in between the prosthesis hand. However, a redesign of the objects can probably lead to more levels of object characteristics that can be used and evaluated. Especially smaller objects and larger weights are required.

To prove the additional value of vibrotactile feedback in daily life grasping tasks, subjects should wear a sensorized prosthetic hand for a longer period. It was shown already that training with a vibrotactile feedback system increases the performance in object displacement tasks [138] and based on theory in motor learning [68] it is expected that task durations of grasping tasks will decrease and require less visual attention with more training.

In previous studies no differences in grasping performance with vibrotactile feedback were found between healthy subjects and subjects with upper limb loss [157, 158], but it should be investigated whether the feedback system is of additional value in daily life grasping tasks for subjects with upper limb loss, the ultimate users of the prosthesis.

\section{Conclusion}

Vibrotactile grasping force and hand aperture feedback is of additional value in object discrimination in situations where visual feedback is not available. However, durations of tasks performed without extensive training were also increased with vibrotactile feedback, which negatively influences the usefulness of the feedback. In daily life grasping tasks no positive nor negative objective effects of the vibrotactile feedback were found, but subjects did experience the feedback as being helpful. Grasping performance was mainly influenced by the order of the tasks, because no extensive training procedure for prosthesis control was followed. 
Chapter 9A - Daily life grasping performance of one amputee

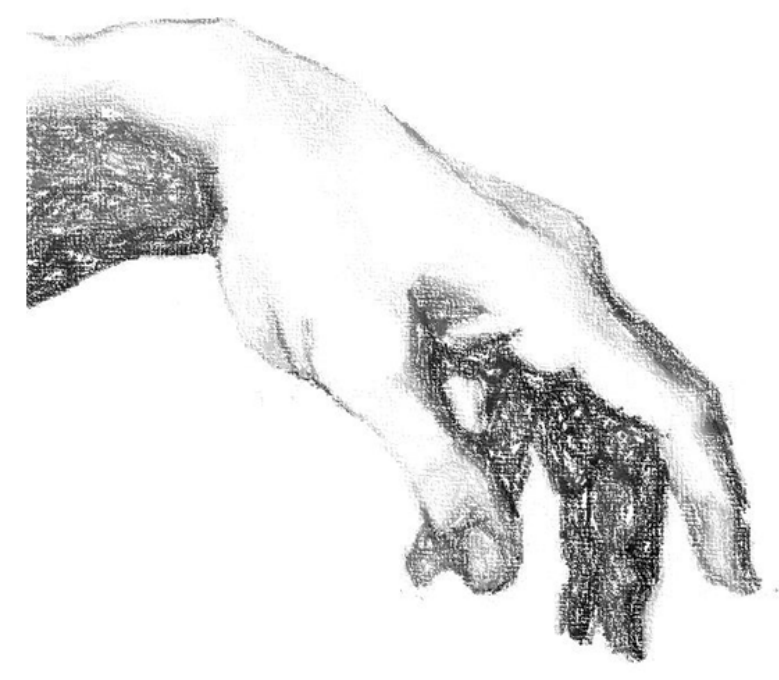

Appendix to: Daily life grasping tasks performed with a myoelectric forearm prosthesis in combination with vibrotactile hand aperture and grasping force feedback

Authors: Heidi Witteveen, Hans Rietman, Peter Veltink

Description of measurements performed on one upper limb loss subject 


\section{Methods}

One upper limb loss subject (46 yrs.), who also participated in an earlier study on vibrotactile feedback, was included in this study. The time after amputation was 2 years, the length of the stump was $23 \mathrm{~cm}$ and the circumference $28 \mathrm{~cm}$. The subject was fitted with a myoelectric forearm prosthesis more than 1 year ago, but indicated that he does not use the prosthesis on a daily basis. Furthermore, he experienced some difficulties with the control of wrist rotation.
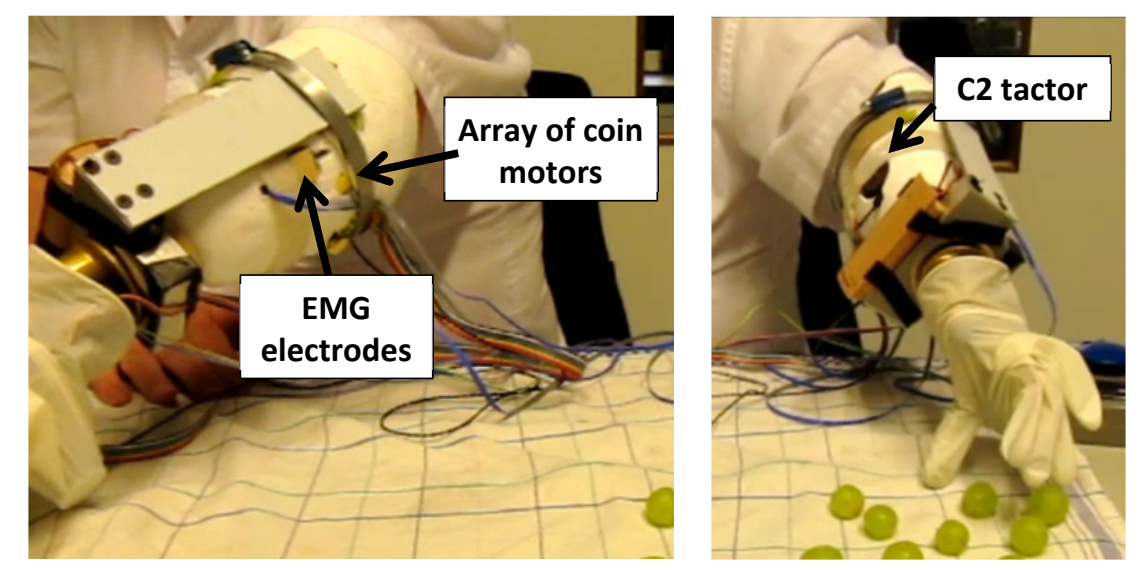

Figure 1: Amputee wearing the plaster prosthetic socket connected to the prosthetic hand. Plastic gloves were used to protect the sensors and the hand. The placement of the array of coin motors, the $\mathrm{C} 2$ tactor and the EMG electrodes is indicated

A subject specific plaster prosthetic socket, which could also be connected to the aluminum frame and the prosthetic hand, was made by an orthopedic engineer. The optimal electrode positions were found by using the PAULA software of Otto Bock and holes were made in the socket at these positions to fit the electrodes from outside (see Figure 1). Furthermore, 8 holes were made to fit the coin motors around the forearm stump and a hole in between the holes for the electrodes was made to fit the $\mathrm{C} 2$ tactor (see Figure 1). The distance between the electrodes and the C2 tactor and between the C2 tactor and coin motors was made as large as possible to diminish possible interference.

\section{Results}

Due to unforeseen circumstances, the time between the fitting of the socket and the actual measurements at the lab was about two months and as a result the socket did not fit very well anymore. It turned out to be very hard for the subject to control the prosthetic hand, using the EMG signal measured from the electrodes in the socket. Most of the time it was only 'binary control', so fast opening and closing of the hand, without control of velocity or grasping force. After more than $1 \frac{1}{2}$ hour, the subject was able to stepwise control the opening and closing of the hand and the subject then performed the grape transfer task. The task was finished within 3 minutes and 15 seconds and the number of successful transferred grapes was 11 , which are both results that are similar to the healthy subjects. After finishing this task, the socket had to be fitted again and afterwards the prosthesis control seemed more difficult again. The grape transfer task 
with additional vibrotactile feedback was started, but almost all grapes were squeezed and therefore, the subject rated the helpfulness of the feedback at 0.1 on the VAS scale. No more measurements were performed anymore, because the subject was very tired.

\section{Discussion}

The main issue during these measurements was the limited control of the prosthesis. Whether this was influenced by the socket that did not fit well or by an overall low level of controllability of the subject cannot be concluded. Bouwsema et al. concluded from a study with healthy subjects on training with a myoelectric forearm prosthesis that part of the subjects were able to use the proportional control, while the other subjects could only open and close the prosthesis [23]. In their study almost $45 \%$ of the subjects could not optimally control the proportional prosthesis, while in our study on healthy subjects, only 1 subject had to be excluded, because he could not proportionally control the prosthesis. However, it is very likely that the upper limb loss subject should also be placed in the subgroup that cannot optimally use the prosthesis. He is fitted with a myoelectric forearm prosthesis, but does not use the prosthesis on a regular basis and also reported some difficulties in prosthesis control.

No conclusions about the usefulness of the feedback could be drawn from these measurements, because the subject was not able to control the prosthesis anymore when the vibrotactile feedback was turned on. This could have been influenced by the interference of the vibrotactile stimulation with the EMG control, which was however not seen in the experiments on healthy subjects where the same equipment was used or by the fact that the subject was overwhelmed by the experience of the vibrotactile stimulation. Furthermore, the socket was loosened and had to be fitted again between both series of experiments, which very likely has influenced the controllability of the prosthesis. Finally, the subject indicated that he was very tired after two hours of experiment, which could have influenced the controllability as well. It was shown possible to integrate both the vibrotactile hand aperture and grasping force feedback in a prosthesis socket and the subject indicated that he could feel the differences between the different coin motors and amplitude levels of the $\mathrm{C} 2$ tactor, but unfortunately it was not possible to investigate whether he was able to interpret and use the feedback during functional grasping tasks. 
9A - Daily life grasping performance of one amputee 


\section{General discussion}

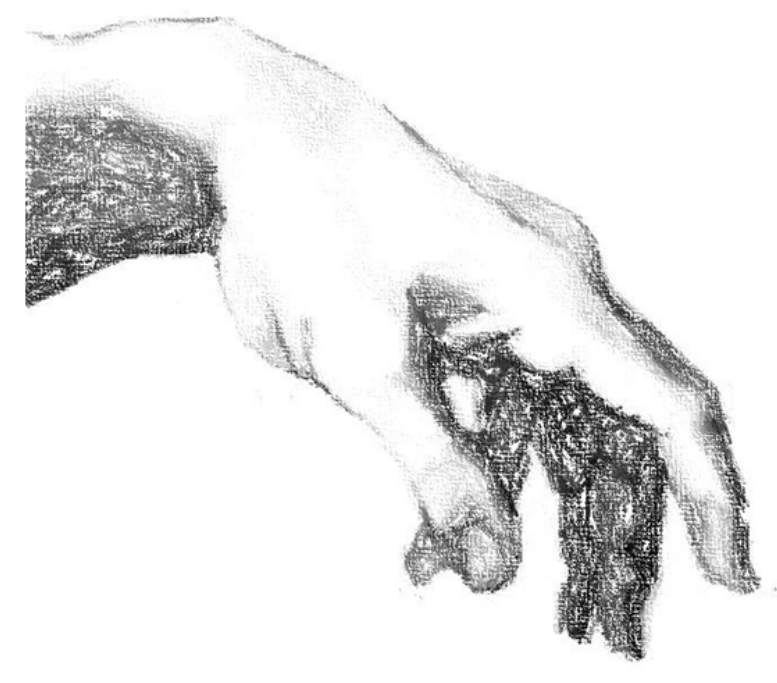


Starting from the feedback requirements for future myoelectric forearm prostheses, derived from the workshop and described in the introduction, several studies have been performed and described in the previous chapters of this thesis. Whether all feedback requirements have been fulfilled and to what extend will be discussed in this general discussion chapter. First the main findings and implications from this thesis will be discussed. After that, directions for future research will be discussed, first focusing on the approach taken in this thesis, but subsequently also on other feedback methods and feedback modalities. Finally the main conclusions from this thesis will be summarized.

\section{Main findings and implications}

In this paragraph the main findings of the several studies of this thesis will be discussed and related to other literature on this topic and the main implications for future research are summarized. First, three topics of the first and second part of the thesis are discussed (vibrotactile vs. electrotactile stimulation, use of coin motor(s) vs. a C2 tactor and effects of stimulation configurations), followed by a discussion on the third part, the clinical evaluation, of the thesis.

\section{Vibrotactile vs. electrotactile stimulation}

Both vibrotactile and electrotactile stimulation were proposed to provide feedback in a non-invasive way. In earlier studies (1960-1980) mostly electrotactile stimulation has been used, while in recent studies the focus is more on vibrotactile stimulation, initiated by the miniaturization of the vibrotactile stimulators. In a paper by Kaczmarek et al. [78], possibilities and pros and cons of both stimulation methods for sensory substitution devices were described, but no direct comparison between both stimulation methods for sensory feedback was performed. In chapter 2 the usability of an array of vibrotactile and electrotactile stimulators to provide hand aperture feedback in virtual grasping tasks was compared. No significant differences in grasping performances were found, but in some cases the task durations were significantly lower with vibrotactile stimulation compared to electrotactile stimulation. One of the major drawbacks of electrotactile stimulation, indicated in review studies of Kaczmarek [78] and Jones [76], is the limited range of stimulation between the sensation and pain threshold. Furthermore, these thresholds are also highly variable between subjects and variable over time, which requires threshold determination for individual subjects, but also tuning of the stimulation during the day. These shortcomings in combination with the found increased task durations with electrotactile stimulation have led to the decision to focus on vibrotactile stimulation for feedback applications in future studies.

\section{Coin motor(s) vs. a C2 tactor}

Two main stimulators have been used in most recent studies on vibrotactile stimulation: (1) a small coin motor or (2) a C2 tactor. The C2 tactor has been specifically developed for vibrotactile stimulation at the human skin, while the coin motors are used more widespread, especially in mobile phones. One of the main advantages of the $\mathrm{C} 2$ tactor is 
the fact that the resonance frequency of the C2 tactor is around $250 \mathrm{~Hz}$, which is within the optimal frequency range for stimulation of important mechanoreceptors (Pacinian corpuscles) in the human skin. Furthermore, the stimulation frequency and amplitude can be controlled independently from each other, which makes them highly suitable for stimulation with a single stimulator. From a preliminary study it was concluded that with a single $\mathrm{C} 2$ tactor at least 6 amplitude or pulse frequency levels could be distinguished [89]. The main advantages of the coin motor over the $\mathrm{C} 2$ tactor are the small size (circular disk of $1 \mathrm{~cm} \times 0.3 \mathrm{~cm}$ compared to $3 \mathrm{cmx} 0.5 \mathrm{~cm}$ ) and the low costs $( \pm 1 \$$ compared to $\$ 250$ ). The stimulation frequencies of the coin motors are lower (between 50 and $150 \mathrm{~Hz}$ ) in comparison to the $\mathrm{C} 2$ tactor and the orientation of stimulation is tangential to the skin instead of perpendicular. Possibly, other mechanoreceptors will be stimulated via the coin motors, but this has not been investigated yet. The main disadvantage of the coin motors is the fact that the frequency and amplitude cannot be controlled independently from each other, which likely limits the possibilities for the use of a single coin motor for vibrotactile feedback. In chapter 1 the mechanical and psychophysical characteristics of stimulation with a single coin motor at three stimulation locations on the forearm have been investigated. It was shown that mechanical characteristics varied largely over the stimulation locations and therefore the range of possible stimulation frequencies was variable as well. No differences in psychophysical characteristics (the number of frequency levels that could be distinguished by the subjects) were found between the stimulation locations, but the number of distinguishable frequency levels was limited (on average) to 2. A way of creating a larger number of distinguishable levels has been proposed in a study by Cipriani et al. where three coin motors are placed on top of each other and constructive interference was created by varying the stimulation frequency [39]. Three amplitude levels could be distinguished successfully and with combinations of stimulator locations and the number of active stimulators, subjects were able to distinguish between 3 hand grasps and 2 force levels of a robotic hand successfully. These results are very promising and should be investigated further, but stimulation patterns should also be kept as simple as possible to keep the required attention for interpretation of the patterns by the subjects as low as possible.

Based on the abovementioned considerations it is concluded that vibrotactile stimulation should not be provided through a single coin motor. However, coin motors can be used in an array of stimulators, providing position-modulated feedback. In another preliminary study no differences in localization performance were found between an array of three coin motors and an array of three C2 tactors [82]. The use of coin motors is preferred in this case, because of the smaller size of the stimulators.

\section{Stimulation configurations}

The choice between the use of a single C2 tactor and an array of coin motors can be based on the feedback that has to be provided. We hypothesized that grasping force is intuitively related to a local change in stimulus sensation and therefore grasping force feedback would be most successful with amplitude or pulse frequency modulation of a 
single C2 tactor. On the other hand we hypothesized that hand aperture is most related to a change in position and therefore hand aperture feedback would be most successful with position modulation through an array of coin motors. However, these hypotheses could not be confirmed by our studies. In chapter 4 the performance in a virtual grasping task with grasping force feedback was compared between amplitude and pulse frequency modulation through a single $\mathrm{C} 2$ tactor and position modulation through an array of coin motors. Pulse frequency modulation scored worst, but grasping performance through amplitude modulation or position modulation was similar. In chapter 5 grasping force feedback via amplitude modulation through a single C2 tactor was combined with hand aperture feedback via an array of coin motors, which resulted in increased grasping performance in comparison to the no feedback situation. However, the control condition with hand aperture feedback via a single $\mathrm{C} 2$ tactor in combination with grasping force feedback via an array of coin motors showed no differences with the supposed optimal feedback configuration and in some cases scored even better. Based on our results, on healthy subjects, we can conclude that both stimulators can be used for hand aperture and grasping force feedback. It can be determined for each situation separately which stimulator to use, depending for example on the available space in the prosthesis socket.

Beforehand it was expected that the orientation of an array of vibrotactile feedback stimulators on the forearm would influence the grasping performance. Studies with electrotactile [70] and tactile stimulation [41] showed that localization performance is better for stimulators placed in a transversal orientation around the arm, which was explained by the asymmetric shape of the receptive fields of the mechanorecepters, which are smaller in the transversal direction. However, the results from chapter 2 did not reveal any differences in grasping performance between hand aperture feedback through transversal or longitudinal oriented arrays of vibrotactile and electrotactile stimulators, for both healthy subjects and upper limb loss subjects. The neighborhood of bony landmarks close to the stimulators can also enhance the localization performance and therefore increase the grasping performance [35]. In chapter 5, again the orientation of an array of coin motors was varied and results in grasping performance were compared, but now stimulators were placed over the elbow joint for the longitudinal orientation, introducing an extra bony landmark. However, this did not influence the grasping performance, because again no differences were found between both array orientations. In future studies and future feedback applications using an array of stimulators, the choice for the orientation is free and can be dependent on the available space in the prosthesis socket and the placement of the EMG electrodes for prosthesis control.

\section{Clinical evaluation}

The optimal stimulation parameters to provide the (vibrotactile) feedback, as discussed in the previous paragraphs, were all derived from studies using a virtual setup controlled by mouse scrolling. To evaluate the possibilities of application of the feedback methods in a real myoelectric forearm prosthesis, experiments in a more realistic setting 
were performed. First, it was investigated if EMG control of the prosthesis would influence the feedback interpretation and thereby the grasping performance. In one of the very few studies on vibrotactile feedback in combination with EMG control, it was concluded that a $\mathrm{C} 2$ tactor could be placed $5 \mathrm{~cm}$ from the EMG electrodes without any interference issues [28]. In the study of chapter 7 an array of coin motors was used to provide hand aperture feedback and grasping performance was compared between mouse scroll input and EMG control. All healthy subjects were able to successfully control the hand opening of the virtual hand and no differences in grasping performance were found. Therefore, it was concluded that EMG control is not influenced by vibrations of the coin motors if they are placed at least $2 \mathrm{~cm}$ from the EMG electrodes and EMG control does not influence the interpretation of the feedback.

Based on literature it could not be determined whether subjects with upper limb loss, the ultimate users of the prosthetic system, would be able to interpret the vibrotactile feedback equally well as healthy subjects. A small number of studies investigated the sensitivity of the stump of amputees, but with contradictive results [24, 66, 73]. Furthermore, no information on the sensitivity of subjects with a congenital defect was available. Therefore, in chapter 2 and 6 the results of the studies on hand aperture and stiffness feedback have been compared between healthy subjects and forearm amputees. No differences in grasping performance and stiffness identification were found. In chapter 8 the studies on hand aperture and grasping force feedback (chapter 2 and 4) were repeated with 10 subjects with upper limb loss. The results from this study were highly similar to the results of chapter 2 and 4 and therefore it was concluded that the feedback can be successfully interpreted by the ultimate users of a myoelectric forearm prosthesis as well. Differences between amputees and subjects with a congenital defect could not be evaluated due to the low number of subjects, but can be investigated in future research, because this could have implications for the development of the feedback system. It may be necessary to develop separate feedback systems for both subject groups to make them optimal for both groups.

In the last research chapter of this thesis (chapter 9) the ultimate application of the feedback system in a myoelectric forearm prosthesis is approached best. After a short training period of about half an hour, healthy subjects performed abstract and daily life grasping tasks, while receiving vibrotactile grasping force and hand aperture feedback. In comparison to conditions without visual feedback, the vibrotactile feedback is clearly of additional value, because the object identification performance is better in comparison the no feedback condition. However, in conditions with visual feedback available no differences in grasping performance were found and visual feedback always outperforms the vibrotactile feedback only condition. This was also seen in a study by Chatterjee et al. [28] where a grasping task had to be performed while receiving vibrotactile grasping force feedback through a single $\mathrm{C} 2$ tactor. Although no objective additional value of the vibrotactile feedback was seen in the daily life grasping tasks, the subjective results indicated that some subjects did experience the feedback as helpful, which was also seen in a study by Cipriani et al. [40]. Furthermore, we expect the 
grasping performance to increase over time and expect that vibrotactile feedback really becomes of additional value after adequate training.

\section{Feedback requirements}

In the introduction of this thesis, the determination of the requirements for a future feedback system for myoelectric forearm prostheses has been described. The requirements were derived via a workshop with physicians and engineers, called representative users, but no actual prosthesis users were involved. The main reason for including physicians and engineers is their broader view on the current and future technological developments in comparison to prosthesis users and their broader view on the shortcomings of current myoelectric forearm prostheses. However, the opinions of the prosthesis users are also very important, because they are using the prosthesis continuously and know what they are missing in their current prosthesis. It would be helpful to work through the workshop questionnaires again with the upper limb loss subjects who participated in our studies, because they now probably have a better idea about what is meant by feedback and how it can be applied.

In a recent study, Lewis et al. [86] investigated the requirements for future feedback applications through questionnaires filled in by users of myoelectric forearm prostheses. Although, the percentage of respondents was low, the feedback requirements were almost similar to those found via the workshop. The most needed improvement, according to Lewis et al., would be the incorporation of grasping force feedback, while proprioceptive feedback about prosthesis movement and position was considered very important as well and rated second and third on their requirements list.

The success of the developed feedback methods can be, amongst others, interpreted from the fulfillment of the requirements derived via the workshop and described in the introduction of this thesis. The five feedback requirements were:

1) Continuous and proportional feedback on grasping force should be provided

2) Position feedback should be provided to the user

3) The stimulation used for feedback should be intuitive and easily interpretable

4) Feedback should be unobtrusive to the user and others

5) Feedback should be adjustable

In chapter 2, 3, 4 and 8 it was shown that requirement 1 and 2 are fulfilled, because in these chapters it was shown that vibrotactile hand aperture and grasping force feedback did significantly improve the grasping performance of healthy subjects as well as upper limb loss subjects. In chapter 5 and 6 it was shown that both feedback methods could also be combined successfully into one system and even provide feedback about object stiffness, which was one of 'nice-to-haves' from the workshop [107].

Requirement 3 stated that the feedback should be intuitive for the users, which has been addressed in chapter 3 of this thesis. The feedback system is completely intuitive if users do not have to think about the interpretation of the feedback and can use it in a 
subconscious way. The methodology of double tasks was used to evaluate the intuitiveness of the vibrotactile hand aperture feedback. The results showed that until a moderate level of distraction, there was no influence of the distraction on the performance in the grasping tasks, even though no training session was included in these experiments. The highest level of distraction (counting and subtracting beeps of different tones) did negatively influence the grasping performance. It is concluded that the vibrotactile (hand aperture) feedback cannot be used completely subconsciously now, but it is expected that with increasing training time, the grasping performance will increase and the interpretation of the feedback will become more and more subconscious. It can be questioned to which activities of daily living the different levels of distraction can be related. If routine daily life tasks are related to moderate levels of distraction, the vibrotactile feedback can still be used and could be of additional value. In chapter 7 it was concluded that that EMG control did not influence the interpretation of the vibrotactile feedback. This finding is also an indication for the fulfillment of the feedback requirement on intuitiveness, because EMG control can be seen as an attention demanding double task.

By the choice for vibrotactile (or electrotactile) stimulation at the start of the research, requirement 4 on the obtrusiveness of the feedback was already fulfilled, because these methods are non-invasive and usually do not interfere with the environment. However, the vibrotactile stimulators can make some noise that could be disturbing for the user or the environment. It should be investigated how these stimulators should be incorporated in the prosthesis socket to reduce the noise.

Requirement 5 on the adjustability of the feedback has not been investigated directly in the presented studies. However, it is shown that the choice for a feedback configuration or vibrotactile stimulator is not fixed and therefore the feedback can be adjusted to the situation and the user. Furthermore, the choice for vibrotactile stimulation gives the opportunity to adjust the amplitude of stimulation to create clearly tangible sensations for every user, without risking painful sensations.

\section{Directions for future research}

In this thesis, the possibilities of vibrotactile hand aperture and grasping force feedback have only been evaluated in short term experiments, lasting maximally two hours, while the ultimate feedback system will be used much longer in daily practice. The effects of long-term vibrotactile stimulation have not been studied extensively. In one study longterm vibrotactile stimulation was performed for four weeks after which no adverse effects were found [6]. Possible problems with long-term stimulation could be the adaptation to the continuous stimuli (fading of the perceived intensity), irritation of the skin, annoyance of the users and the energy efficiency of the feedback system. Vibrotactile stimulation seems to be less sensitive to adaptation in comparison to electrotactile stimulation [153], but still the perceived amplitude of stimulation can fade over time, which makes the feedback less pronounced and less useful. To reduce the effects of adaptation, intermittent stimulation can possibly be used, which has shown to 
be successful for electrotactile stimulation [26]. Furthermore, the changing activation of the coin motors within an array would already diminish the adaptation effects.

Another way to reduce the problems with long-term continuous stimulation, could be the use of discrete feedback, which can for example mean that feedback is only provided when the state of the prosthesis changes. The whole human sensory system is also based on changing sensory information. However, when the prosthetic hand is not used for a certain period of time, the user would not be able to determine the state of hand at that moment. Therefore, it should be investigated which kind of feedback is preferred by the users and a trade-off should be made between the use of continuous and discrete feedback.

The training that was incorporated in the described studies of this thesis was relatively short, maximally half an hour, but still the positive effects of the feedback could be demonstrated. One of the requirements was that the feedback should be intuitive, implying a short training period, which has been achieved in our studies. However, it has been proven in other studies on (vibrotactile) feedback that longer periods of training can significantly improve the performance with the feedback $[28,119,138]$. Not only the duration of training, but also the used training method can influence the performance with a myoelectric forearm prosthesis [22]. It should be investigated what the optimal training procedure must be, because a training period that is too long will also negatively affect the acceptance of the prosthesis.

Another aspect that is very important, but not investigated in this thesis, is the time delay of the feedback. It can be questioned whether the feedback is fast enough to enable the user to react in time. Especially when slippage of objects has to be prevented, which is more or less a reflex, time delays are important. Future research should investigate the maximal time allowed to prevent slippage and whether is possible to provide feedback and react on this feedback within the time limit. In a recent review on feedback for myoelectric forearm prostheses [10], timing requirements were specified: the temporal delays from sensory detection to interpretation by the user should not exceed $300 \mathrm{msec}$. to create a feeling of embodiment of the prosthesis. Furthermore, the maximal time for information transmission from the sensor to the skin of the user should be between 3-5 msec. to be in the range of the transmission speed of the healthy human sensory system. It should be further investigated whether these requirements can be met when using vibrotactile stimulation. It is likely that a more discrete feedback signal is required to immediately grasp the attention of the user, which probably should also be more pronounced than a vibrotactile stimulus. For other movements, like the closing and opening of the hand, time delays are less important, because the velocity of these movements is limited as well.

Evaluation of the (vibrotactile) feedback in this thesis mainly has focused on the performance in virtual grasping tasks. Also in other studies manipulators or robots, executing virtual or abstract grasping tasks, were used to describe performance instead of evaluations in daily life grasping tasks performed with a real myoelectric prosthesis. Performance in daily life tasks should be evaluated in future research to really prove the advantages of the feedback. These evaluations should not only focus on the objective 
results, but user experiences should also be incorporated, because they will decide whether they want to use the feedback system or not.

This thesis on feedback for myoelectric forearm prostheses was part of the Myopro project, which aimed for the development of an innovative and intuitive myoelectric forearm prosthesis. To reach this goal, research has also been performed on the sensing of user intention, the high and low level control of the prosthesis, the development of an underactuated prototype prosthetic hand and a virtual training system. All of these aspects will have some interaction with the feedback system, which has to be evaluated in future research. Within the sensing part, the desired grasping force will be estimated from the amplitude of the EMG recordings. It should be investigated whether eight different levels of grasping force can be controlled, otherwise the number of feedback levels should be adjusted. The sensors used in the mechanical prototype to derive force and position information are different from those used in the feedback experiments. Therefore, it should also be investigated whether the correct information is transmitted to the feedback system. Furthermore, all components of the Myopro project should be incorporated in the prosthetic hand and socket, which is a challenge that might require adjustments to all components.

Within the Myopro project several grasps and wrist movements of the prosthesis are defined, while within this thesis the feedback was based on an old-fashion prosthetic hand that can only open and close the hand with a tripod grasp. It should be investigated if the hand aperture and grasping force information is the same for all grasps and if the feedback should be the same for all grasps or that adjustments in the feedback system should be made. Furthermore, a state machine is used to control the mechanical hand and it can be questioned whether the user would benefit from feedback about the current state of the state machine, for example feedback about the grasp type that is being executed.

\section{Other feedback methods than electrotactile and vibrotactile stimulation}

The focus of this thesis has been on vibrotactile stimulation to provide the feedback, but other feedback methods could be useful as well. In the chapters on hand aperture and grasping force feedback already some work on skin stretch and pressure feedback has been described. Especially pressure feedback is promising, because there is no modality mismatch and the pressure measured at the fingertips can be directly applied, with the same amplitude, to the skin. However, some work is necessary to further minimize the actuators that are required to make them applicable in the prosthesis socket.

Other non-invasive feedback methods can be visual and auditory feedback, which have a better spatial and temporal resolution respectively in comparison to tactile feedback. However, one of the main requirements for the feedback system was that it should be unobtrusive to the environment, which cannot be achieved when using visual or auditory feedback. Auditory feedback has been proposed by González et al. [61] and Vargas et al. [147] to assist in the learning phase of prosthesis use or in combination with electrotactile feedback. These applications of auditory feedback should be further 
investigated, because auditory feedback could be very helpful for these training purposes, which also holds for visual feedback.

A last non-invasive feedback method to be described here is the use of tendon vibrations to provide proprioceptive feedback. By applying high amplitude vibrations to the skin just above muscle tendons, proprioceptive sensations can be evoked by stimulation of the muscle spindles. These methods seem promising to provide for example feedback about wrist and hand movements [122], but more research is necessary on the required stimulation parameters and the possibilities of miniaturization of the stimulators.

A feedback or control method on the edge of non-invasive and invasive methods is the use of cineplasty, where the control muscles are mechanically coupled to the prosthesis [151]. This is one of the best examples of extended proprioception [134], because the muscle activation is directly coupled to the movement of the prosthesis. However, applications in practice are rarely seen, likely due to cosmetic reasons.

Besides the use of non-invasive stimulation, direct nerve or cortical stimulation can also be used to provide feedback. Promising results have already been published by Horch and Dhillon on direct nerve stimulation $[50,71]$ and by Berg et al. on intracortical stimulation [17]. To our opinion, this will be the future of feedback applications in prostheses, but still requires decades of research to optimize the stimulation. Noninvasive feedback was one of the feedback requirements, but in future prostheses it is very likely that direct nerve recordings can be used for prosthesis control and therefore one surgical procedure can be enough to implant both the recording and stimulation electrodes.

One of the most promising developments in prosthesis research of the last years is the procedure of targeted reinnervation as developed by Kuiken et al. [84] By redirecting the forearm nerves to the chest muscles, user intentions of hand movements can be easily recorded by EMG measurements. An unexpected feature of this procedure was the reinnervation of the overlying skin, which resulted in sensations of touch of the fingers when touching the reinnervated skin of the chest. Future research is necessary to further explore these phenomena and to find the optimal ways to use these channels to provide feedback. It should be mentioned that targeted reinnervation is especially useful in above elbow amputations, because subjects with below elbow amputations still have most of the muscles that are necessary for the control of the hand.

\section{Other feedback modalities than hand aperture and grasping force feedback}

\section{Slip feedback}

One of the main reasons for including grasping force feedback was to prevent object slippage. Heavier objects, with the same roughness, would require a larger grasping force to prevent slippage and grasping force feedback will provide information about the applied grasping force to the prosthesis user. However, to successfully hold an object, they need to combine this information with information about the weight of the object. If this information is not available or incorrect, grasping force feedback would not be sufficient to prevent slippage. A way to avoid slippage of objects with unknown weights 
is to apply higher grasping forces, which is not energy efficient and also not suitable for fragile objects. Feedback about object slippage would not require preliminary information of the object weight. However, sensing of object slippage is rather difficult. Several sensing methods have been described in literature [27, 42, 120], but these methods are either too bulky to be implemented on a prosthetic fingertip or not sensitive enough in all possible situations of daily use of a prosthesis.

Another disadvantage of the use of slip feedback at the skin can be the time delay from the occurrence of slip and the detection to the stimulation and eventually the reaction of the user. Therefore, the best option for now would be to incorporate automatic slip control, which has already been applied in commercial prostheses as the I-limb [5] and research applications as the Smarthand project [38]. However, the disadvantage would be that the user loses control over the prosthesis, which can negatively influence the embodiment of the prosthesis and ultimately result in rejection of the prosthesis. In future research it should be investigated if and how slip feedback should be provided to the user of the prosthesis and a trade-off between the level of control by the user and the level of automatic control by the prosthesis controller should be made. To our opinion, the user should be in control as much as possible, because this will enhance the acceptance of the prosthesis.

\section{Temperature feedback}

An important aspect in object discrimination, when sight on the prosthesis is blocked, is the temperature of the object. A plausible way to provide feedback about the temperature of an object would be thermo feedback, where the temperature of the skin changes with changing object temperatures. A first exploration of thermo feedback has been described by Davalli et al. [46]. Although, object discrimination can be improved with thermo feedback, research is not focusing on this aspect, because thermo feedback is only indicated as a 'nice-to-have'. 


\section{Conclusions}

This thesis is a next step towards the implementation of a sensory feedback system in a myoelectric forearm prosthesis. As a first step, the requirements for the optimal feedback system were derived. It was shown that both hand aperture and grasping force feedback could be provided successfully through vibrotactile stimulation and can be combined into one feedback system. This combination of hand aperture and grasping force feedback also provided stiffness information. Furthermore, it was shown that the use of vibrotactile hand aperture feedback did not require a high level of user attention and that the influence of the EMG control on the usefulness of the hand aperture feedback was minimal. Finally, we showed that vibrotactile feedback is also applicable for subjects with upper limb loss, the ultimate users of the feedback and that the feedback is, to some extent, useful in daily life grasping tasks.

To make a next step towards the implementation in daily life prostheses, further research is necessary, among others, on the long term effects of stimulation, the optimal training procedures, evaluation of timing aspects, the incorporation of the feedback in the prosthesis socket, the combination with EMG control and further miniaturization of the stimulators. 


\section{References}

[1] (2003). Statistics hand prostheses. Available:

http://biomed.brown.edu/Courses/BI108/BI108_2003_Groups/Hand_Prosthetic s/stats.html

[2] (2010). Statistics hand prostheses in the netherlands. Available: www.kortmaarkrachtig.com

[3] (2012). Ottobock. Available: www.ottobock.com

[4] (2012). Rslsteeper. Available: http://rslsteeper.com

[5] (2012). Touch bionics inc. Available: http://www.touchbionics.com

[6] Alles D.S., Information transmission by phantom sensations, IEEE Transactions on Man-Machine Systems, vol. 11, pp. 85-91, 1970.

[7] Anani A. and Korner L., Discrimination of phantom hand sensations elicited by afferent electrical nerve stimulation in below-elbow amputees, Medical Progress through Technology, vol. 6, pp. 131-5, 1979.

[8] Antfolk C., Balkenius C., Lundborg G., et al., A tactile display system for hand prostheses to discriminate pressure and individual finger localization, Journal of Medical and Biological Engineering, vol. 30, pp. 355-359, 2010.

[9] Antfolk C., D'Alonzo M., Controzzi M., et al., Artificial redirection of sensation from prosthetic fingers to the phantom hand map on transradial amputees: Vibrotactile versus mechanotactile sensory feedback, IEEE Transactions on Neural Systems and Rehabilitation Engineering, vol. 21, pp. 112-120, 2013.

[10] Antfolk C., D'Alonzo M., Rosen B., et al., Sensory feedback in upper limb prosthetics, Expert Review of Medical Devices, vol. 10, pp. 45-54, 2013.

[11] Asghari Oskoei $\mathrm{M}$. and $\mathrm{Hu}$ H., Myoelectric control systems--a survey, Biomedical Signal Processing and Control, vol. 2, pp. 275-294, 2007.

[12] Atkins D.J., Heard D.C.Y., and Donovan W.H., Epidemiologic overview of individuals with upper-limb loss and their reported research priorities, Journal of Prosthetics and Orthotics, vol. 8, pp. 2-11, 1996.

[13] Babkoff $H_{\text {., }}$ Electrocutaneous psychophysical input-output functions and temporal integration, Perception \& Psychophysics, vol. 23, pp. 251-257, 1978.

[14] Bach-y-Rita P. and Kercel S.W., Sensory substitution and the human-machine interface, Trends in Cognitive Sciences, vol. 7, pp. 541-546, 2003.

[15] Bark C., Wheeler J., and Premakumar S., Comparison of skin stretch and vibrotactile stimulation for feedback of proprioceptive information, in Proceedings of the IEEE/VR Symposium on Haptic Interfaces, 2008.

[16] Beeker T.W., During J., and Denherto A., Artificial touch in a hand-prosthesis, Medical \& Biological Engineering, vol. 5, pp. 47-49, 1967.

[17] Berg J.A., Dammann J.F., Tenore F.V., et al., Behavioral demonstration of a somatosensory neuroprosthesis, IEEE Transactions on Neural Systems and Rehabilitation Engineering, vol. 21, pp. 500-507, 2013. 
[18] Biddiss E. and Chau T., Upper-limb prosthetics - critical factors in device abandonment, American Journal of Physical Medicine \& Rehabilitation, vol. 86, pp. 977-987, 2007.

[19] Blank A., Okamura A.M., and Kuchenbecker K.J., Effects of proprioceptive motion feedback on sighted and non-sighted control of a virtual hand prosthesis, in Proceedings of the Symposium on haptic interfaces for virtual environments and teleoperator systems, Reno, Nevada, USA, 2008.

[20] Blank A., Okamura A.M., and Kuchenbecker K.J., Identifying the role of proprioception in upper-limb prosthesis control: Studies on targeted motion, Transactions on Applied Perception, vol. 7, pp. 1-23, 2010.

[21] Botvinick M. and Cohen J., Rubber hands 'feel' touch that eyes see, Nature, vol. 391, pp. 756-756, 1998.

[22] Bouwsema H., van der Sluis C.K., and Bongers R.M., The role of order of practice in learning to handle an upper-limb prosthesis, Archives of Physical Medicine and Rehabilitation, vol. 89, pp. 1759-1764, 2008.

[23] Bouwsema H., van der Sluis C.K., and Bongers R.M., Learning to control opening and closing a myoelectric hand, Archives of Physical Medicine and Rehabilitation, vol. 91, pp. 1442-1446, 2010.

[24] Braune S. and Schady W., Changes in sensation after nerve injury or amputation - the role of central factors, Journal of Neurology Neurosurgery and Psychiatry, vol. 56, pp. 393-399, 1993.

[25] Brookhuis R.A., Lammerink T.S.J., Wiegerink R.J., et al., 3d force sensor for biomechanical applications, Sensors and Actuators A: Physical, vol. 182, pp. 28$33,2012$.

[26] Buma D.G., Buitenweg J.R., and Veltink P.H., Intermittent stimulation delays adaptation to electrocutaneous sensory feedback, IEEE Transactions on Neural Systems and Rehabilitation Engineering, vol. 15, pp. 435-441, 2007.

[27] Chappell P.H., Making sense of artificial hands, Journal of Medical Engineering and Technology, vol. 35, pp. 1-18, 2011.

[28] Chatterjee A., Chaubey P., Martin J., et al., Testing a prosthetic haptic feedback simulator with an interactive force matching task, Journal of Prosthetics and Orthotics, vol. 20, pp. 27-34 2008.

[29] Chatterjee A., Chaubey P., Martin J., et al., Quantifying prosthesis control improvements using a vibrotactile representation of grip force, in Proceedings of the IEEE Region 5 Conference, 2008, pp. 1-5.

[30] Cheng A., Nichols K.A., Weeks H.M., et al., Conveying the configuration of a virtual human hand using vibrotactile feedback, in Proceedings of the IEEE Haptics Symposium (HAPTICS), 2012, pp. 155-162.

[31] Cheung B., Van Erp J.B.F., and Cholewiak R.W., Anatomical, neurophysiological and perceptual issues of tactile perception in Tactile Displays for Orientation, Navigation and Communication in Air, Sea and Land Environments NATO Research and Technology organisation, 2008. 
[32] Childress D.S., Closed-loop control in prosthetic systems - historical perspective, Annals of Biomedical Engineering, vol. 8, pp. 293-303, 1980.

[33] Cholewiak R.W. and Beede K., The representation of space through static and dynamic tactile displays, in Proceedings of the Virtual reality international conference in conjunction with the human computer interaction, Las Vegas, NE, USA, 2005.

[34] Cholewiak R.W., Brill J.C., and Schwab A., Vibrotactile localization on the abdomen: Effects of place and space, Perception \& Psychophysics, vol. 66, pp. 970-987, 2004.

[35] Cholewiak R.W. and Collins A.A., Vibrotactile localization on the arm: Effects of place, space, and age, Perception \& Psychophysics, vol. 65, pp. 1058-1077, 2003.

[36] Chouvardas V.G., Miliou A.N., and Hatalis M.K., Tactile displays: Overview and recent advances, Displays, vol. 29, pp. 185-194, 2008.

[37] Cipriani C., Antfolk C., Balkenius C., et al., A novel concept for a prosthetic hand with a bidirectional interface: A feasibility study, IEEE Transactions on Biomedical Engineering, vol. 56, pp. 2739-2743, 2009.

[38] Cipriani C., Controzzi M., and Carrozza M.C., The smarthand transradial prosthesis, Journal of Neuroengineering and Rehabilitation, vol. 8, 2011.

[39] Cipriani C., D'Alonzo M., and Carrozza M.C., A miniature vibrotactile sensory substitution device for multifingered hand prosthetics, IEEE Transactions on Biomedical Engineering, vol. 59, pp. 400-408, 2012.

[40] Cipriani C., Zaccone F., Micera S., et al., On the shared control of an emgcontrolled prosthetic hand: Analysis of user-prosthesis interaction, Ieee Transactions on Robotics, vol. 24, pp. 170-184, 2008.

[41] Cody F.W.J., Garside R.A.D., Lloyd D., et al., Tactile spatial acuity varies with site and axis in the human upper limb, Neuroscience Letters, vol. 433, pp. 103-108, 2008.

[42] Cotton D.P.J., Chappell P.H., Cranny A., et al., A novel thick-film piezoelectric slip sensor for a prosthetic hand, IEEE Sensors Journal, vol. 7, pp. 752-761, 2007.

[43] D'Alonzo M., Cipriani C., and Carrozza M.C., Vibrotactile sensory substitution in multi-fingered hand prostheses: Evaluation studies, in Proceedings of the IEEE International Conference on Rehabilitation Robotics (ICORR), 2011, pp. 1-6.

[44] Damian D.D., Arieta A.H., Martinez H., et al., Slip speed feedback for grip force control, IE Transactions on Biomedical Engineering, vol. 59, pp. 2200-2210, 2012.

[45] Datta D., Selvarajah K., and Davey N., Functional outcome of patients with proximal upper limb deficiency - acquired and congenital, Clinical Rehabilitation, vol. 18, pp. 172-177, 2004.

[46] Davalli A., Sacchetti R., Fanin S., et al., Biofeedback for upper limb myoelectric prostheses, Technology and Disability, vol. 13, pp. 161-172, 2001. 
[47] de Winter J.C.F. and Dodou D., Five-point likert items: T-test versus mannwhitney-wilcoxon, Practical Assessment, Research \& Evaluation, vol. 15, p. 1, 2010.

[48] Der G. and Deary I.J., Age and sex differences in reaction time in adulthood: Results from the united kingdom health and lifestyle survey, Psychology and Aging, vol. 21, pp. 62-73, 2006.

[49] Desmond D.M., Coping, affective distress, and psychosocial adjustment among people with traumatic upper limb amputations, Journal of Psychosomatic Research, vol. 62, pp. 15-21, 2007.

[50] Dhillon G.S. and Horch K.W., Direct neural sensory feedback and control of a prosthetic arm, IEEE Transactions on Neural Systems and Rehabilitation Engineering, vol. 13, pp. 468-472, 2005.

[51] Di Pino G., Guglielmelli E., and Rossini P.M., Neuroplasticity in amputees: Main implications on bidirectional interfacing of cybernetic hand prostheses, Progress in Neurobiology, vol. 88, pp. 114-126, 2009.

[52] Diller T.T. and Srinivasan M.A., Frequency response of human skin in vivo to mechanical stimulation, Technical report, Massachusetts Institute of Technology 2001.

[53] Dudkiewicz I., Gabrielov R., Seiv-Ner I., et al., Evaluation of prosthetic usage in upper limb amputees, Disability and Rehabilitation, vol. 26, pp. 60-63, 2004.

[54] Ehrsson H.H., Rosen B., Stockselius A., et al., Upper limb amputees can be induced to experience a rubber hand as their own, Brain, vol. 131, pp. 34433452, 2008.

[55] Fan R.E., Culjat M.O., King C.H., et al., A haptic feedback system for lower-limb prostheses, IEEE Transactions on Neural Systems and Rehabilitation Engineering, vol. 16, pp. 270-277, 2008.

[56] Gaiser I.N., Pylatiuk C., Schulz S., et al., The fluidhand III: A multifunctional prosthetic hand, Journal of Prosthetics and Orthotics, vol. 21, pp. 91-96, 2009.

[57] Gasson M., Hutt B., Goodhew I., et al., Invasive neural prosthesis for neural signal detection and nerve stimulation, International Journal of Adaptive Control and Signal Processing, vol. 19, pp. 365-375, 2005.

[58] Gescheider G.A., Psychophysics: Method, theory, and application, second ed.: L. Erlbaum Associates, 1985.

[59] Gillespie R.B., Contreras-Vidal J.L., Shewokis P.A., et al., Toward improved sensorimotor integration and learning using upper-limb prosthetic devices, in Proceedings of the Annual International Conference of the IEEE Engineering in Medicine and Biology Society, 2010, pp. 5077-5080.

[60] Goff G.D., Rosner B.S., Detre T., et al., Vibration perception in normal man and medical patients, Journal of Neurology Neurosurgery and Psychiatry, vol. 28, pp. 503-509, 1965.

[61] Gonzalez J., Yu W.W., and Arieta A.H., Multichannel audio biofeedback for dynamical coupling between prosthetic hands and their users, Industrial Robot-an International Journal, vol. 37, pp. 148-156, 2010. 
[62] Green B.G., The perception of distance and location for dual tactile pressures, Perception \& Psychophysics, vol. 31, pp. 315-323, 1982.

[63] Gulati R.J. and Srinivasan M.A., Human fingerpad under indentation i: Static and dynamic force response, in Proceedings of the 1995 Bioengineering conference, Beaver Creek, Colorado, USA, 1995, pp. 261-262.

[64] Gurari N., Kuchenbecker K.J., and Okamura A.M., Stiffness discrimination with visual and proprioceptive cues, in Proceedings of the World Haptics 2009: Third Joint Eurohaptics Conference and Symposium on Haptic Interfaces for Virtual Environment and Teleoperator Systems, 2009, pp. 121-126.

[65] Gurari N., Smith K., Madhav M., et al., Environment discrimination with vibration feedback to the foot, arm, and fingertip, in Proceedings of the IEEE 11th International Conference on Rehabilitation Robotics, Vols 1 and 2, 2009, pp. 398-403.

[66] Haber W.B., Reactions to loss of limb: Physiological and psychological aspects, Annals of the New York Academy of Sciences, vol. 74, pp. 14-24, 1958.

[67] Hahn J.F., Vibrotactile adaptation and recovery measured by 2 methods, Journal of Experimental Psychology, vol. 71, pp. 655-658, 1966.

[68] Halsband U. and Lange R.K., Motor learning in man: A review of functional and clinical studies, Journal of Physiology-Paris, vol. 99, pp. 414-424, 2006.

[69] Haugland M.K., Hoffer J.A., and Sinkjaer T., Skin contact force information in sensory nerve signals recorded by implanted cuff electrodes, IEEE Transactions on Rehabilitation Engineering, vol. 2, pp. 18-28, 1994.

[70] Higashiyama A. and Hayashi M., Localization of electrocutaneous stimuli on the fingers and forearm - effects of electrode configuration and body axis, Perception \& Psychophysics, vol. 54, pp. 108-120, 1993.

[71] Horch K., Meek S., Taylor T.G., et al., Object discrimination with an artificial hand using electrical stimulation of peripheral tactile and proprioceptive pathways with intrafascicular electrodes, IEEE Transactions on Neural Systems and Rehabilitation Engineering, vol. 19, pp. 483-489, 2011.

[72] Huang H.J. and Mercer V.S., Dual-task methodology: Applications in studies of cognitive and motor performance in adults and children, Pediatric Physical Therapy, vol. 13, pp. 133-140, 2001.

[73] Hunter J.P., Katz J., and Davis K.D., Dissociation of phantom limb phenomena from stump tactile spatial acuity and sensory thresholds, Brain, vol. 128, pp. 308-320, 2005.

[74] Hunter J.P., Katz J., and Davis K.D., Stability of phantom limb phenomena after upper limb amputation: A longitudinal study, Neuroscience, vol. 156, pp. 939949, 2008.

[75] Johansson R.S. and Westling G., Roles of glabrous skin receptors and sensorimotor memory in automatic control of precision grip when lifting rougher or more slippery objects, Experimental Brain Research, vol. 56, pp. $550-564,1984$. 
[76] Jones L.A. and Safter N.B., Tactile displays: Guidance for their design and application, Human Factors, vol. 50, pp. 90-111, 2008.

[77] Kaczmarek K.A., Kramer K.M., Webster J.G., et al., A 16-channel 8-parameter waveform electrotactile stimulation system, IEEE Transactions on Biomedical Engineering, vol. 38, pp. 933-943, 1991.

[78] Kaczmarek K.A., Webster J.G., Bach-y-Rita P., et al., Electrotactile and vibrotactile displays for sensory substitution systems, IEEE Transactions on Biomedical Engineering, vol. 38, pp. 1-16, 1991.

[79] Kandel E.R., Schwartz J.H., and Jessell T.M., Principles of neural science, fourth ed.: McGraw-Hill, Health Professions Division, 2000.

[80] Ki-Uk K., Minseung A., Dong-Soo K., et al., Perceptual and biomechanical frequency response of human skin: Implication for design of tactile displays, in Proceedings of the First Joint Eurohaptics Conference and Symposium on Haptic Interfaces for Virtual Environment and Teleoperator Systems, World Haptics 2005, pp. 96-101.

[81] Kim K., Colgate J.E., Santos-Munne J.J., et al., On the design of miniature haptic devices for upper extremity prosthetics, IEEE-ASME Transactions on Mechatronics, vol. 15, pp. 27-39, 2010.

[82] Knoop T.H., Vibrotactiele lokalisatie op de onderarm: De invloed van tactortype, oriëntatie en tussenafstand, Bachelor thesis, Biomedical Signals and Systems, University of Twente, 2011.

[83] Kuchenbecker K.J., Gurari N., and Okamura A.M., Effects of visual and proprioceptive motion feedback on human control of targeted movement, in Proceedings of the IEEE 10th international conference on rehabilitation robotics, Noordwijk, The Netherlands, 2007.

[84] Kuiken T.A., Marasco P.D., Lock B.A., et al., Redirection of cutaneous sensation from the hand to the chest skin of human amputees with targeted reinnervation, Proceedings of the National Academy of Sciences of the United States of America, vol. 104, pp. 20061-20066, 2007.

[85] Langley G.B. and Sheppeard H., The visual analogue scale: Its use in pain measurement, Rheumatology International, vol. 5, pp. 145-148, 1985.

[86] Lewis S., Russold M.F., Dietl H., et al., User demands for sensory feedback in upper extremity prostheses, in Proceedings of the IEEE International Symposium on Medical Measurements and Applications Proceedings (MeMeA), 2012, pp. 1-4.

[87] Light C.M., Chappell P.H., Hudgins B., et al., Intelligent multifunction myoelectric control of hand prostheses, Journal of Medical Engineering \& Technology, vol. 26, pp. 139-146, 2002.

[88] Light C.M., Chappell P.H., and Kyberd P.J., Establishing a standardized clinical assessment tool of pathologic and prosthetic hand function: Normative data, reliability, and validity, Archives of Physical Medicine and Rehabilitation, vol. 83, pp. 776-783, 2002. 
[89] Luft F., A vibrotactile feedback system to provide simultaneous position and force feedback from a transradial hand prosthesis, Master thesis, Biomedical Signals and Systems, University of Twente, Enschede, 2012.

[90] Lundborg G. and Rosen B., Sensory substitution in prosthetics, Hand Clinics, vol. 17, pp. 481-488, 2001.

[91] Lundstrom R., Local vibrations mechanical impedance of the human hands glabrous skin, Journal of Biomechanics, vol. 17, pp. 137-144, 1984.

[92] Mahns D.A., Perkins N.M., Sahai V., et al., Vibrotactile frequency discrimination in human hairy skin, Journal of Neurophysiology, vol. 95, pp. 1442-1450, 2006.

[93] Mann R.W. and Reimers S.D., Kinesthetic sensing for emg controlled boston arm, Ieee Transactions on Man-Machine Systems, vol. 11, pp. 110-115, 1970.

[94] Marasco P.D., Kim K., Colgate J.E., et al., Robotic touch shifts perception of embodiment to a prosthesis in targeted reinnervation amputees, Brain, vol. 134, pp. 747-758, 2011.

[95] Marcus P.L. and Fugluvand A.J., Perception of electrical and mechanical stimulation of the skin: Implications for electrotactile feedback, Journal of Neural Engineering, vol. 6, 2009.

[96] Meek S.G., Jacobsen S.C., and Goulding P.P., Extended physiologic taction: Design and evaluation of a proportional force feedback system, Journal of Rehabilitation Research and Development, vol. 26, pp. 53-62, 1989.

[97] Moore C.E.G., Partner A., and Sedgwick E.M., Cortical focusing is an alternative explanation for improved sensory acuity on an amputation stump, Neuroscience Letters, vol. 270, pp. 185-187, 1999.

[98] Moore T.J. and Mundie J.R., Measurement of specific mechanical impedance of skin - effects of static force, site of stimulation, area of probe, and presence of a surround, Journal of the Acoustical Society of America, vol. 52, pp. 577-583, 1972.

[99] Mortimer B.J.P., Zets G.A., and Cholewiak R.W., Vibrotactile transduction and transducers, Journal of the Acoustical Society of America, vol. 121, pp. 2970-2977, 2007.

[100] Murray A.M., Klatzky R.L., and Khosla P.K., Psychophysical characterization and testbed validation of a wearable vibrotactile glove for telemanipulation, Presence-Teleoperators and Virtual Environments, vol. 12, pp. 156-182, 2003.

[101] O'Boyle D.J., Moore C.E.G., Poliakoff E., et al., Human locognosic acuity on the arm varies with explicit and implicit manipulations of attention: Implications for interpreting elevated tactile acuity on an amputation stump, Neuroscience Letters, vol. 305, pp. 37-40, 2001.

[102] Oakley I., Yeongmi K., Junhun L., et al., Determining the feasibility of forearm mounted vibrotactile displays, in Proceedings of the 14th Symposium on Haptic Interfaces for Virtual Environment and Teleoperator Systems, 2006, pp. 27-34.

[103] Oppenheim A.V., Willsky A.S., and Young I.T., Signals and systems, first ed.: Prentice-Hall, 1983. 
[104] Panarese A., Edin B.B., Vecchi F., et al., Humans can integrate force feedback to toes in their sensorimotor control of a robotic hand, IEEE Transactions on Neural Systems and Rehabilitation Engineering, vol. 17, pp. 560-567, 2009.

[105] Parker P., Englehart K., and Hudgins B., Myoelectric signal processing for control of powered limb prostheses, Journal of Electromyography and Kinesiology, vol. 16, pp. 541-548, 2006.

[106] Patterson P.E. and Katz J.A., Design and evaluation of a sensory feedback system that provides grasping pressure in a myoelectric hand, Journal of Rehabilitation Research and Development, vol. 29, pp. 1-8, 1992.

[107] Peerdeman B., Boere D., Witteveen H.J.B., et al., Myoelectric forearm prostheses: State of the art from a user-centered perspective, Journal of Rehabilitation Research and Development, vol. 48, pp. 719-738, 2011.

[108] Petrie S., Collins J.G., Solomonow M., et al., Mechanoreceptors in the human elbow ligaments, Journal of Hand Surgery-American Volume, vol. 23A, pp. 512518, 1998.

[109] Pfeiffer E.A., Electrical stimulation of sensory nerves with skin electrodes for research diagnosis communication and behavioral conditioning - a survey, Medical \& Biological Engineering, vol. 6, pp. 637-\&, 1968.

[110] Phillips C.A., Sensory feedback-control of upper-extremity and lowerextremity motor prostheses, CRC Critical Reviews in Biomedical Engineering, vol. 16, pp. 105-140, 1988.

[111] Plettenburg D.H. and Herder J.L., Voluntary closing: A promising opening in hand prosthetics, Technology and Disability, vol. 15, pp. 85-94, 2003.

[112] Pons J.L., Ceres R., Rocon E., et al., Virtual reality training and emg control of the manus hand prosthesis, Robotica, vol. 23, pp. 311-317, 2005.

[113] Pons J.L., Ceres R., Rocon E., et al., Objectives and technological approach to the development of the multifunctional manus upper limb prosthesis, Robotica, vol. 23, pp. 301-310, 2005.

[114] Prior R.E. and Lyman J., Electrocutaneous feedback for artificial limbs. Summary progress report. February 1, 1974, through july 31, 1975, Bulletin of prosthetics research, pp. 3-37, 1975.

[115] Prior R.E., Lyman J., Case P.A., et al., Supplemental sensory feedback for the va/nu myoelectric hand. Background and preliminary designs, Bulletin of prosthetics research, pp. 170-91, 1976.

[116] Pylatiuk C., Kargov A., and Schulz S., Design and evaluation of a low-cost force feedback system for myoelectric prosthetic hands, Journal of Prosthetics and Orthotics, vol. 18, pp. 57-61, 2006.

[117] Pylatiuk C., Mounier S., Kargov A., et al., Progress in the development of a multifunctional hand prosthesis, in Proceedings of the 26th Annual International Conference of the IEEE Engineering in Medicine \& Biology Society, San Fransisco, USA, 2004. 
[118] Pylatiuk C., Schulz S., and Döderlein L., Results of an internet survey of myoelectric prosthetic hand users, Prosthetics and Orthotics International, vol. 31, pp. 362-370, 2007.

[119] Qi A., Matsuoka Y., and Stepp C.E., Multi-day training with vibrotactile feedback for virtual object manipulation, in Proceedings of the IEEE International Conference on Rehabilitation Robotics (ICORR), 2011, pp. 1-5.

[120] Rodriguez-Cheu L.E. and Casals A., Sensing and control of a prosthetic hand with myoelectric feedback, in Proceedings of the The First IEEE/RAS-EMBS International Conference on Biomedical Robotics and Biomechatronics (BioRob), 2006, pp. 607-612.

[121] Rohland T.A., Sensory feedback for powered limb prostheses, Medical \& Biological Engineering, vol. 13, pp. 300-301, 1975.

[122] Roll J.P. and Gilhodes J.C., Proprioceptive sensory codes mediating movement trajectory perception: Human hand vibration-induced drawing illusions, Canadian Journal of Physiology and Pharmacology, vol. 73, pp. 295-304, 1995.

[123] Saunders I. and Vijayakumar S., The role of feed-forward and feedback processes for closed-loop prosthesis control, Journal of Neuroengineering and Rehabilitation, vol. 8, 2011.

[124] Schmidl H., The importance of information feedback in prostheses for the upper limbs, Prosthetics and Orthotics International, vol. 1, pp. 21-4, 1977.

[125] Schmidt R.A., Motor control and learning - a behavioral emphasis, second ed. Champaign, Illinois: Human Kinetics Publishers, Inc., 1988.

[126] Schultz A.E., Marasco P.D., and Kuiken T.A., Vibrotactile detection thresholds for chest skin of amputees following targeted reinnervation surgery, Brain Research, vol. 1251, pp. 121-129, 2009.

[127] Scott R.N., Brittain R.H., Caldwell R.R., et al., Sensory-feedback system compatible with myoelectric control, Medical \& Biological Engineering \& Computing, vol. 18, pp. 65-69, 1980.

[128] Self B.P., Van Erp J.B.F., Eriksson L., et al., Human factors issues of tactile displays for military environments in Tactile Displays for Orientation, Navigation and Communication in Air, Sea and Land Environments NATO Research and Technology organisation, 2008.

[129] Sensinger J.W., Schultz A.E., and Kuiken T.A., Examination of force discrimination in human upper limb amputees with reinnervated limb sensation following peripheral nerve transfer, IEEE Transactions on Neural Systems and Rehabilitation Engineering, vol. 17, pp. 438-444, 2009.

[130] Shannon G.F., Comparison of alternative means of providing sensory feedback on upper limb prostheses, Medical \& Biological Engineering, vol. 14, pp. 289-294, 1976.

[131] Shannon G.F., Myoelectrically-controlled prosthesis with sensory feedback, Medical \& Biological Engineering \& Computing, vol. 17, pp. 73-80, 1979. 
[132] Sherrick C.E., Cholewiak R.W., and Collins A.A., The localization of lowfrequency and high-frequency vibrotactile stimuli, Journal of the Acoustical Society of America, vol. 88, pp. 169-179, 1990.

[133] Sherwood L., Human physiology: From cells to systems, fourth ed.: Brooks/Cole, Cengage Learning, 2001.

[134] Simpson D.C., The choice of control system for the multimovement prosthesis: Extended physiological proprioception, in The control of upperextremity prostheses and orthoses, P. Herberts, Ed., ed Springfield (IL): Thomas, 1974, pp. 146-150.

[135] Solomonow M., Lyman J., and Freedy A., Electro-tactile 2-point discrimination as a function of frequency, body site, laterality, and stimulation codes, Annals of Biomedical Engineering, vol. 5, pp. 47-60, 1977.

[136] Srinivasan M.A. and Lamotte R.H., Tactual discrimination of softness, Journal of Neurophysiology, vol. 73, pp. 88-101, 1995.

[137] Stepp C. and Matsuoka Y., Vibrotactile sensory substitution for object manipulation: Amplitude versus pulse train frequency modulation, IEEE Transactions on Neural Systems and Rehabilitation Engineering, vol. PP, pp. 1-1, 2011.

[138] Stepp C.E., An Q., and Matsuoka Y., Repeated training with augmentative vibrotactile feedback increases object manipulation performance, Plos One, vol. 7, 2012.

[139] Stepp C.E., Chang C., Malhotra M., et al., Vibrotactile feedback aids emg control of object manipulation, in Proceedings of the Annual International Conference of the IEEE Engineering in Medicine and Biology Society (EMBC), 2011, pp. 10611064.

[140] Stepp C.E. and Matsuoka Y., Relative to direct haptic feedback, remote vibrotactile feedback improves but slows object manipulation, in Proceedings of the Annual International Conference of the IEEE Engineering in Medicine and Biology Society (EMBC), 2010, pp. 2089-2092.

[141] Stepp C.E. and Matsuoka Y., Vibrotactile sensory substitution for object manipulation: Amplitude versus pulse train frequency modulation, Ieee Transactions on Neural Systems and Rehabilitation Engineering, vol. 20, pp. 31-37, 2012.

[142] Stevens J.C. and Choo K.K., Spatial acuity of the body surface over the life span, Somatosensory and motor research, vol. 13, pp. 153-166, 1996.

[143] Szeto A.Y.J. and Lyman J., Comparison of codes for sensory feedback using electro-cutaneous tracking, Annals of Biomedical Engineering, vol. 5, pp. 367383, 1977.

[144] Tan H.Z., Durlach N.I., Beauregard G.L., et al., Manual discrimination of compliance using active pinch grasp - the roles of force and work cues, Perception \& Psychophysics, vol. 57, pp. 495-510, 1995. 
[145] Tashiro T. and Higashiyama A., The perceptual properties of electrocutaneous stimulation - sensory quality, subjective intensity, and intensity-duration relation, Perception \& Psychophysics, vol. 30, pp. 579-586, 1981.

[146] Teuber H., Krieger H.P., and Bender M.B., Reorganization of sensory function in amputation stumps - 2-point discrimination, Federation Proceedings, vol. 8, pp. 156-156, 1949.

[147] Vargas J.G., Yu W.W., and Ieee, Audio aided electro-tactile perception training for finger posture biofeedback, in 30th annual international conference of the IEEE Engineering in Medicine and Biology Society, 2008, pp. 4230-4233.

[148] Verrillo R.T., Psychophysics of vibrotactile stimulation, Journal of the Acoustical Society of America, vol. 77, pp. 225-232, 1985.

[149] Wang G., Zhang X., Zhang J., et al., Gripping force sensory feedback for a myoelectrically controlled forearm prosthesis, in Proceedings of the IEEE International Conference on Systems, Man, and Cybernetics, Vancouver, Canada, 1995, pp. 501-504.

[150] Weinstein S., Intensive and extensive aspects of tactile sensititivity as a function of body part, sex, and laterality, in The skin senses, D. Kenshalo, Ed., ed Springfield, Il.: Charles C. Thomas, 1968, pp. 195-222.

[151] Weir R.F., Heckathorne C.W., and Childress D.S., Cineplasty as a control input for externally powered prosthetic components, Journal of Rehabilitation Research and Development, vol. 38, pp. 357-363, 2001.

[152] Weiss T., Miltner W.H.R., Adler T., et al., Decrease in phantom limb pain associated with prosthesis-induced increased use of an amputation stump in humans, Neuroscience Letters, vol. 272, pp. 131-134, 1999.

[153] Wentink E.C., Mulder A., Rietman J.S., et al., Vibrotactile stimulation of the upper leg: Effects of location, stimulation method and habituation, in Proceedings of the 2011 Annual International Conference of the IEEE Engineering in Medicine and Biology Society, 2011, pp. 1668-1671.

[154] Westling G. and Johansson R.S., Factors influencing the force control during precision grip, Experimental Brain Research, vol. 53, pp. 277-284, 1984.

[155] Wheeler J., Bark K., Savall J., et al., Investigation of rotational skin stretch for proprioceptive feedback with application to myoelectric systems, Ieee Transactions on Neural Systems and Rehabilitation Engineering, vol. 18, pp. 58-66, 2010.

[156] Witteveen H.J.B., de Rond L., Rietman J.S., et al., Hand-opening feedback for myoelectric forearm prostheses: Performance in virtual grasping tasks influenced by different levels of distraction, Journal of Rehabilitation Research and Development, vol. 49, pp. 1517-1526, 2012.

[157] Witteveen H.J.B., Droog E.A., Rietman J.S., et al., Vibro- and electrotactile user feedback on hand opening for myoelectric forearm prostheses, IEEE Transactions on Biomedical Engineering, vol. 59, pp. 2219-2226, 2012. 
[158] Witteveen H.J.B., Luft F., Rietman J.S., et al., Stiffness feedback for myoelectric forearm prostheses using vibrotactile stimulation, IEEE Transactions on Neural Systems and Rehabilitation Engineering, vol. PP, pp. 1-1, 2013.

[159] Witteveen H.J.B., Rietman J.S., and Veltink P.H., Grasping force and slip feedback through vibrotactile stimulation to be used in myoelectric forearm prostheses, in Proceedings of the Annual International Conference of the IEEE Engineering in Medicine and Biology Society (EMBC), 2012, pp. 29692972.

[160] Wright D.L. and Kemp T.L., The dual-task methodology and assessing the attentional demands of ambulation with walking devices, Physical Therapy, vol. 72, pp. 306-315, 1992.

[161] Yang D.-p., Zhao J.-d., Gu Y.-k., et al., An anthropomorphic robot hand developed based on underactuated mechanism and controlled by emg signals, Journal of Bionic Engineering, vol. 6, pp. 255-263, 2009.

[162] Zafar M. and Van Doren C.L., Effectiveness of supplemental grasp-force feedback in the presence of vision, Medical \& Biological Engineering \& Computing, vol. 38, pp. 267-274, 2000. 


\section{Summary}

Myoelectric forearm prostheses offer their users an increasing level of functionality, but still no sensory feedback is incorporated in these prostheses, while it is shown that sensory feedback is essential for optimal grasping control of the prosthesis and increases the embodiment of the prosthesis, which improves the user acceptance. Through a workshop with clinicians and engineers, requirements for a future feedback system for myoelectric forearm prostheses were derived and described in the introduction chapter of this thesis. The main requirements were: (1) feedback about grasping force should be provided, (2) hand aperture feedback should be provided, (3) the feedback should be intuitive and easily interpretable, (4) the feedback should be unobtrusive and (5) the feedback should be adjustable.

A possible stimulator to provide the feedback is a coin motor that is mainly used in mobile phones and is small and cheap in comparison to other vibrotactile stimulators. To investigate the usability of one single coin motor to provide feedback, psychophysical and mechanical characteristics have been derived for three measurement locations on the forearm (chapter 1). Mechanical characteristics did vary over the three locations, but psychophysical characteristics did not. The number of stimulation levels that could be distinguished (psychophysical measurements) was approximately two, which is not very useful for feedback purposes. Therefore, it was concluded that coin motors should be used within an array of stimulators.

Two main non-invasive stimulation methods are vibrotactile and electrotactile stimulation. Electrotactile stimulation has been used more in earlier research, but no direct comparison between both methods was made before. In chapter 2 , both methods were used to provide hand aperture feedback. In a virtual setup, hand aperture was controlled via mouse scrolling and hand aperture feedback was provided through an array of eight vibrotactile or electrotactile stimulators. Grasping performance was defined as the percentages correctly applied hand apertures to hold a presented virtual object. No differences in grasping performance between both stimulation methods were found, except for a longer task duration with electrotactile stimulation. Based on these results and the fact that the stimulation range between the sensation and pain threshold for electrotactile stimulation is limited, it was decided to use vibrotactile stimulation in future studies.

To investigate the intuitiveness of the vibrotactile hand aperture feedback through an array of eight coin motors, a study involving double tasks has been performed (chapter 3). While counting beeps, subjects had to perform the virtual grasping task. No differences in grasping performance were found up to a moderate level of distraction, but with a highly distractive task, subjects could not use the vibrotactile feedback anymore. Therefore, it is concluded that the vibrotactile feedback cannot be used fully subconsciously.

Optimal stimulation parameters to provide vibrotactile hand aperture and grasping force feedback have been investigated in several studies. For hand aperture feedback, 
longitudinal and transversal orientations of an array of stimulators on the forearm have been compared (chapter 2 and 5). For grasping force feedback the use of single C2 tactor and an array of coin motors has been compared (chapter 4 and 5). No differences in grasping performance, in a virtual setup, between both array orientations and between both stimulators were found. Therefore, a situation specific choice for configurations and stimulators can be made, depending for example on the available space in the socket.

Hand aperture and grasping force feedback should be ultimately combined in one system, which has been investigated in two studies. In the first study hand aperture feedback was kept constant during object holding, simulating stiff objects (chapter 5), while in the second study hand aperture feedback was also provided during object holding (chapter 6). Results of the first study showed that in a virtual setup, controlled by mouse scrolling, grasping performance increases with vibrotactile feedback and no adverse interference between both feedback methods were found. In the second study, the possibility of deriving stiffness information from the combination of vibrotactile hand aperture and grasping force feedback was investigated. Subjects were able to correctly determine the stiffness of a virtual object out of four options in $60 \%$ of the cases.

The possibilities of the ultimate application of vibrotactile feedback in a myoelectric forearm prosthesis have been investigated in several studies in the last part of this thesis. EMG control has been excluded in the first studies, because it would require too much training, but EMG control will be used in the eventual application. Therefore, we investigated the influence of the EMG control on the usability of the vibrotactile feedback and vice versa (chapter 7). Although EMG control would require more attention from the user, which resulted in longer task durations, it did not negatively influence the grasping performance with the vibrotactile feedback.

The majority of the subjects in all studies were healthy subjects, while subjects with upper limb loss will be the ultimate users of the system. Therefore, the vibrotactile feedback methods for vibrotactile hand aperture and grasping force feedback have been investigated on ten subjects with upper limb loss as well (chapter 8). Also in this study, vibrotactile feedback did improve the grasping performance and results were comparable with the results from the studies on healthy subjects.

The final study of this thesis describes the application of vibrotactile hand aperture and grasping force feedback in a commercially available myoelectric forearm prosthesis while performing daily life grasping tasks (chapter 9). It was shown that vibrotactile feedback is of additional value in object discrimination when visual feedback is blocked, but when visual feedback is available as well no additional value of the feedback was found, although a majority of the subjects did rate the feedback as moderately helpful.

Most of the feedback requirements, as described in the introduction chapter, have been fulfilled by vibrotactile feedback about hand aperture and grasping force. The feedback methods are rather intuitive and not obtrusive to the environment. However, still further research is necessary to develop a feedback system that will be incorporated in the myoelectric forearm prostheses. At least, training effects and the influence of time 
Summary

delays should be further investigated and more studies in realistic settings, with subjects using a myoelectric prosthesis for a longer period, are required. 


\section{Samenvatting}

De functionaliteit van myoelektrische onderarmprotheses verbetert gestaag, maar nog altijd wordt in geen van deze protheses sensorische terugkoppeling naar de gebruiker toegepast, terwijl uit verschillende studies blijkt dat het geven van sensorische terugkoppeling essentieel is voor het optimaal kunnen aansturen van de prothese. Bovendien vergroot deze terugkoppeling de acceptatie van de prothese door de gebruiker. Aan de hand van de uitkomsten van een workshop met clinici en technici zijn eisen opgesteld voor een toekomstig systeem voor sensorische terugkoppeling. Deze eisen zijn beschreven in het introductie hoofdstuk van dit proefschrift. De belangrijkste eisen zijn: (1) er moet sensorische terugkoppeling plaatsvinden over de grijpkracht, (2) de mate van handopening van de prothese moet teruggekoppeld worden naar de gebruiker, (3) de sensorische terugkoppeling moet intuïtief en makkelijk te interpreteren zijn, (4) de sensorische feedback moet niet storend zijn voor de omgeving en de gebruiker en (5) de sensorische terugkoppeling moet instelbaar zijn.

Een 'coin motortje' dat vooral gebruikt wordt in mobiele telefoons zou als vibrotactiele stimulator gebruikt kunnen worden om sensorische terugkoppeling te geven. Deze stimulator is klein en goedkoop in vergelijking met andere vibrotactiele stimulatoren. Om de bruikbaarheid van een enkele coin motor voor het leveren van sensorische terugkoppeling te onderzoeken, zijn psychofysische en mechanische eigenschappen van het systeem bepaald voor drie locaties op de onderarm (hoofdstuk 1). De mechanische eigenschappen verschillenden over de drie locaties, maar er waren geen verschillen in de psychofysische eigenschappen. Het aantal stimulatieniveaus dat onderscheiden kon worden (psychofysica) door de proefpersonen was gemiddeld twee en dat is niet erg bruikbaar voor het geven van sensorische terugkoppeling. Daarom luidt de conclusie dat coin motors alleen maar gebruikt kunnen worden in een array met meerdere stimulatoren.

Twee belangrijke methodes om non-invasief te stimuleren zijn vibrotactiele en elektrotactiele stimulatie. In vroegere studies werd voornamelijk gebruik gemaakt van elektrotactiele stimulatie, maar een uitgebreide vergelijking van beide methodes is nooit uitgevoerd. In hoofdstuk $\mathbf{2}$ worden beide methodes gebruikt om sensorische terugkoppeling over de handopening te geven. In een virtuele omgeving werd de handopening aangestuurd door middel van scrollen met een computermuis, terwijl sensorische terugkoppeling werd gegeven door middel van een array van acht vibrotactiele of elektrotactiele stimulatoren. De grijpprestaties werden uitgedrukt in de percentages correct uitgevoerde handopeningen die nodig waren om virtuele objecten van verschillende groottes vast te pakken. Er zijn geen verschillen in grijpprestaties gevonden tussen beide stimulatiemethodes, uitgezonderd een langere taakduur met elektrotactiele stimulatie. Op basis van deze resultaten en het feit dat het stimulatiebereik tussen de gevoelsdrempel en de pijndrempel voor elektrotactiele stimulatie beperkt is, is besloten om met vibrotactiele feedback verder te gaan. 
Om te evalueren hoe intuïtief de sensorische terugkoppeling van handopening door middel van een array van vibrotactiele stimulatoren is, is een studie met dubbeltaken uitgevoerd (hoofdstuk 3). Proefpersonen moesten een virtuele grijptaak uitvoeren en ondertussen piepjes van verschillende toonhoogtes optellen en aftrekken. Tot op een gemiddeld niveau van afleiding was er geen verschil in grijpprestaties, maar bij het hoogste niveau van afleiding konden de proefpersonen de vibrotactiele feedback niet meer gebruiken. Dit leidde tot de conclusie dat de sensorische terugkoppeling door middel van vibrotactiele stimulatie niet volledig onderbewust gebruikt kan worden.

In verschillende studies zijn de optimale stimulatieparameters voor het geven van sensorische terugkoppeling over de handopening en grijpkracht onderzocht. Voor de terugkoppeling van handopening is het verschil tussen een longitudinale of transversale oriëntatie van de vibrotactiele stimulatie array onderzocht (hoofdstuk 2 en 5). Het gebruik van een enkele $\mathrm{C} 2$ tactor of een array met coin motors is vergeleken voor de terugkoppeling van grijpkracht (hoofdstuk 4 en 5). Er werden geen verschillen in grijpprestaties gevonden tussen beide oriëntaties van de arrays en tussen beide stimulatoren. Daarom kan geconcludeerd worden dat voor elke situatie de best passende configuratie en stimulator gekozen kan worden op basis van bijvoorbeeld de beschikbare ruimte in de prothesekoker.

Terugkoppeling van handopening en grijpkracht moet uiteindelijk gecombineerd worden in één systeem. De mogelijkheden hiervoor zijn onderzocht in twee studies. In de eerste studie is de terugkoppeling van handopening constant gehouden tijdens het vasthouden van objecten, waardoor het vastgrijpen van stijve objecten gesimuleerd werd (hoofdstuk 5). In de tweede studie was er ook terugkoppeling van handopening tijdens het vasthouden van objecten (hoofdstuk 6). De resultaten van de eerste studie lieten zien dat de grijpprestaties in een virtuele omgeving verbeterd worden met vibrotactiele terugkoppeling en dat er geen sprake was van negatieve interferentie tussen beide stimulatiemethodes. De mogelijkheden om stijfheid informatie terug te koppelen door de combinatie van vibrotactiele terugkoppeling van handopening en grijpkracht zijn onderzocht in de tweede studie. Proefpersonen bleken in staat om in $60 \%$ van de gevallen de stijfheid van een virtueel object correct in te schatten.

In het laatste gedeelte van dit proefschrift is de mogelijke toepassing van de verschillende methodes voor sensorische terugkoppeling in een myoelektrische onderarmprothese verder onderzocht. In eerdere studies was het gebruik van EMG aansturing uitgesloten, omdat dit teveel training van de proefpersonen vergt. EMG aansturing zal echter wel gebruikt worden in de toekomstige protheses en daarom is het effect van EMG aansturing op de bruikbaarheid van de vibrotactiele terugkoppeling en vice versa onderzocht (hoofdstuk 7). Hoewel de EMG aansturing meer aandacht vergt van de gebruiker, wat resulteerde in een langere taakduur, had dit geen negatief effect op de grijpprestaties met vibrotactiele terugkoppeling.

In de beschreven studies zijn voornamelijk gezonde proefpersonen gebruikt, terwijl amputatiepatiënten of patiënten met een aangeboren afwijking van de onderarm de uiteindelijke gebruikers van het systeem zullen zijn. Daarom zijn de vibrotactiele methodes voor het terugkoppelen van handopening en grijpkracht ook geëvalueerd bij 
tien proefpersonen met een amputatie of aangeboren afwijking van de onderarm (hoofdstuk 8). Ook de resultaten van deze studie laten zien dat grijpprestaties verbeteren met sensorische terugkoppeling. Daarnaast werden er geen verschillen met gezonde proefpersonen gevonden.

In de laatste studie van dit proefschrift wordt de toepassing van vibrotactiele terugkoppeling van handopening en grijpkracht in een commercieel verkrijgbare myoelektrische onderarmprothese beschreven (hoofdstuk 9). Diverse grijptaken, vergelijkbaar met taken uit het dagelijks leven, moesten uitgevoerd worden. De resultaten laten zien dat vibrotactiele terugkoppeling van toegevoegde waarde is bij het onderscheiden van verschillende objecten wanneer visuele terugkoppeling geblokkeerd is. Wanneer visuele terugkoppeling wel beschikbaar is, werd geen directe toegevoegde waarde gevonden, maar een meerderheid van de proefpersonen beoordeelde de vibrotactiele terugkoppeling wel als bruikbaar in het uitvoeren van de grijptaken.

Aan de meeste eisen voor de sensorische terugkoppeling, zoals beschreven in het introductie hoofdstuk, is voldaan door gebruik te maken van de beschreven methodes voor vibrotactiele terugkoppeling van handopening en grijpkracht. De methodes zijn redelijk intuïtief en niet storend voor de omgeving. $\mathrm{Er}$ is echter nog wel vervolgonderzoek nodig om te komen tot een systeem voor sensorische terugkoppeling dat daadwerkelijk geïmplementeerd wordt in een myoelektrische onderarmprothese. Met name het effect van training en de invloed van tijdsvertragingen zal verder onderzocht moeten worden. Verder zullen meer studies uitgevoerd moeten worden met proefpersonen die gedurende een langere periode een myoelektrische prothese met de terugkoppelingsmethodes gebruiken in taken die vergelijkbaar zijn met taken uit het dagelijks leven. 


\section{Dankwoord}

Na ruim 4 jaar schrijven, schrappen en schaven is dan nu de afronding van mijn boekje in zicht, maar niet voordat ik iedereen bedankt heb die op welke manier dan ook een bijdrage hieraan hebben geleverd.

Als eerste wil ik mijn promotoren bedanken die mij de mogelijkheid hebben gegeven om aan mijn promotie te beginnen en tot een goed einde te brengen. Peter bedankt voor de dagelijkse begeleiding! Ondanks een drukke agenda was het altijd mogelijk om binnen te lopen en was er tijd om artikelen door te lezen en van commentaar te voorzien. Hans bedankt voor je klinische input in het proces. De combinatie van de techneut en de dokter leverde vaak bijzondere en levendige, maar ook zeker waardevolle discussies op.

Mijn promotiewerk was onderdeel van een groter project en ik denk dat ik het erg getroffen heb met het Myopro project en met name met de projectcollega's. Ons gezamenlijke artikel vormde de basis voor mijn proefschrift en was naar mijn mening ook het begin van een uitstekende samenwerking. Daphne en Bart, ik heb onze samenwerking als zeer waardevol en bijzonder ervaren. Ook de andere projectgenoten wil ik bedanken voor de leerzame en vaak ook erg gezellige Myopro-overleggen die we in de loop der jaren gehad hebben.

Fijne projectgenoten zijn belangrijk, maar misschien nog veel belangrijker zijn de mensen waar je dagelijks mee te maken hebt. Dus collega's bij BSS: enorm bedankt voor de fantastische tijd die ik bij jullie gehad heb. De meelevendheid en gezelligheid tijdens lunchwandelingen en koffiepauzes heb ik enorm gewaardeerd. Extra ondersteuning en begrip kwam vaak van de BSS collega-aio's. De lunches buiten de deur en de Van der Poel ijscoupes waren uitgelezen momenten om promotiedips en frustraties met elkaar te delen. Heel specifiek wil ik ook mijn kamergenoten bedanken voor de mooie tijd die we samen gehad hebben. Betty, Karin, Peter, Josien, Victor, en Eva, jullie waren er altijd; wanneer het even wat minder ging en de verwensingen door de kamer vlogen of wanneer de gezelligheid weer iets te gortig werd. Bedankt voor de radioreclamemeningen, tandenborstelbeestjes, stroopwafels, pepermuntballen en de Heinmomenten. Ed en Marcel bedankt voor alle technische hulp en natuurlijk ook voor de gezelligheid op de gang. Wies bedankt voor alle broodnodige administratieve en vooral sociale ondersteuning!

Gedurende mijn promotie heb ik een aantal studenten mogen begeleiden tijdens hun bachelor- of masteropdrachten. Leonie, Tom, Frauke, Lisette, Ingrid, Frank en Simone bedankt voor al het werk dat jullie voor mij hebben willen doen, ook dankzij jullie is dit boekje er gekomen. Ik heb met jullie allemaal, ondanks alle verschillende persoonlijkheden, heel prettig kunnen werken en wens jullie allemaal heel veel succes met jullie carrières. 
Ook de 'buren' van Biomedische Werktuigbouw wil ik op deze plaats bedanken voor het uitlenen van soms hele opstellingen en het leveren van technische hulp. Inmiddels ben ik zelf onderdeel van deze groep en kan ik jullie ook bedanken voor de fijne ontvangst.

Zonder welwillende proefpersonen had mijn onderzoek nooit een succes kunnen worden. Daarom wil ik graag iedereen bedanken die toch geheel vrijwillig heeft willen meewerken aan een onbekend onderzoek, uitgevoerd door een (voor sommigen) onbekend persoon, zonder daar zelf voordeel bij te hebben. Jeroen Olsman en Hans Konter bedankt voor jullie medewerking bij het vinden en benaderen van geschikte proefpersonen. Daarnaast zouden de laatste metingen niet mogelijk geweest zijn zonder alle medewerking vanuit het RRT (Roessingh Revalidatie Techniek) en met name van Jeroen bij het vervaardigen van de patiënt-specifieke socket en de aanschaf van de myoprothese.

Verder wil ik ook een aantal mensen bedanken die aan de basis van mijn wetenschappelijke carrière hebben gestaan. De tijd tijdens mijn afstuderen en de 18 maanden daarna bij het RRD heb ik als heel bijzonder ervaren en zijn zeker vormend geweest voor mij als onderzoeker. In het bijzonder denk ik hierbij aan de mensen die mij destijds begeleid hebben. Gerlienke, Laura, Judith en Hermie, jullie hebben mij laten ontdekken waartoe ik in staat was, waar ik mij verder in kon ontwikkelen en hebben mij daarbij op het juiste pad gezet. Dank!

Mijn dank gaat natuurlijk ook uit naar diegenen die mij vandaag ondersteund hebben als paranimfen, maar eigenlijk al vanaf de start van mijn studie erbij zijn. Eva en Martijn, al vanaf het begin van onze BMT-opleiding waren we vaak projectgenoten en het contact is, ondanks korte onderbrekingen i.v.m. stages en afstuderen, gebleven mede doordat we alle drie een promotie begonnen aan de UT. Ik hoop dat we elkaar nog vaak zullen zien.

Hoe gezellig en goed de werksituatie ook kan zijn, zonder een prettige thuissituatie wordt het niets, vandaar ook dat mijn grootste dank uitgaat naar de mensen die mij altijd thuis opgevangen hebben: Pap, mam en JP bedankt! En daarnaast waren er nog velen die ook de tijd buiten mijn promotie om tot een fijne periode gemaakt hebben: mensen in Enschede en Beekbergen en omstreken bedankt voor jullie gezelligheid!

Tot slot heeft dit hele promotietraject me ook buiten alle wetenschappelijke hoogtepunten om nog iets fantastisch opgeleverd: Frodo, wat een geluk dat ik je heb mogen leren kennen en dat jij met mij door het leven wilt gaan. Bedankt voor je nooit aflatende steun tijdens de laatste periode van mijn promotie. Ik hoop nog lang en gelukkig met je op te mogen trekken.

Heidi Witteveen 


\section{Biography}

Heidi Witteveen was born in Apeldoorn, the Netherlands, on the 30 th of December 1983. In 2007 she finished her study BioMedical Engineering at the University of Twente. Her master assignment was about the assessment of spasticity in Spinal Cord Injury patients through long-term EMG measurements, which she performed at the Roessingh Research and Development (RRD) in Enschede. Afterwards, she worked at the RRD as a junior researcher on several projects on spasticity assessment and EMG measurements. In August 2009 she started her PhD at the Biomedical Signals and Systems group of the University of Twente. Her PhD work was part of the Myopro project and involved the development and evaluation of tactile feedback methods for myoelectric forearm prostheses.

Currently, Heidi works as a postdoctoral researcher and project manager of the Symbitron project at the Biomechanical Engineering group of the University of Twente. Her research involves the development and evaluation of methods to provide sensory substitution to Spinal Cord Injury patients using a wearable exoskeleton. 


\section{List of publications}

\section{Journal papers}

Witteveen H.J.B., Rietman J.S., Veltink P.H. - Vibrotactile grasping force and hand aperture feedback for myoelectric forearm prosthesis users - accepted for publication in Prosthetics and Orthotics International, 2014

Witteveen H.J.B., Luft F., Rietman J.S., Veltink P.H. - Stiffness feedback for myoelectric forearm prostheses using vibrotactile stimulation - IEEE Transactions on Neural Systems and Rehabilitation Engineering, vol. 22, no. 1, 2014

Witteveen H.J.B., de Rond L., Rietman J.S., Veltink P.H. - Hand opening feedback for myoelectric forearm prostheses; Performance in virtual grasping tasks, influenced by different levels of distraction - Journal of Rehabilitation Research and Development, vol. 49 , no. 10,2012

Witteveen H.J.B., Droog A., Rietman J.S., Veltink P.H. - Vibro- and electrotactile user feedback on hand opening for myoelectric forearm prostheses - IEEE Transactions on Biomedical Engineering, vol. 59, no. 8, 2012

Peerdeman B., Boere D., Witteveen H.J.B., Huis in 't Veld R., Hermens H.J., Stramigioli S., Rietman J.S., Veltink P.H., Misra S. - Myoelectric forearm prostheses: State of the art from a user-centered perspective - Journal of Rehabilitation Research and Development, vol. 48,2011

Witteveen H.J.B., Rietman J.S., Veltink P.H. - The combination of vibrotactile hand aperture and grasping force feedback - submitted

Witteveen H.J.B., Rietman J.S., Veltink P.H. - Vibrotactile feedback about hand aperture of a virtual forearm prosthesis controlled by EMG - submitted 


\section{Conference proceedings and abstracts}

Witteveen H.J.B., Luft F., Rietman J.S., Veltink P.H. - Stiffness feedback by the combination of vibrotactile hand opening and grasping force feedback - 4th Dutch BME conference, 2013, Egmond aan Zee

Witteveen H.J.B., Rietman J.S., Veltink P.H. - Hand opening and grasping force feedback through vibrotactile stimulation for users of myoelectric forearm prostheses - ISPO 2013 World Congress, 2013, Hyderabad, India

Witteveen H.J.B., Rietman J.S., Veltink P.H. - Grasping force and slip feedback through vibrotactile stimulation to be used in myoelectric forearm prostheses - 34th Annual International Conference of the IEEE Engineering in Medicine and Biology Society, 2012, San Diego, USA

Witteveen H.J.B., Knoop T., Rietman J.S., Veltink P.H. - Effects of stimulator type, array orientation and inter-stimulator distance on localization performance with vibrotactile stimulation - Annual symposium of the IEEE EMBS Benelux Chapter, 2011, Brussels, Belgium

Witteveen H.J.B., Rietman J.S., Veltink P.H. - Skin characteristics and psychophysical aspects of vibrotactile stimulation for feedback in forearm prostheses - 3rd Dutch BME conference, 2011, Egmond aan Zee

Van Baal D.W., Witteveen H.J.B., Kallenberg L.A.C., Hermens H.J., Rietman J.S. - A Multichannel sEMG method for control of a forearm prosthesis - 4th Annual Symposium of the IEEE-EMBS Benelux chapter, 2009, Enschede

Witteveen H.J.B., van Weering M.G, Kallenberg L.A.C., Vollenbroek-Hutten M., Hermens H.J., - Muscle activation patterns of chronic low back pain patients during daily living 2nd Dutch BME Conference, 2009, Egmond aan Zee 
Publication list 